\title{
Nazarov Cyclizations Catalyzed by BINOL Phosphoric Acid Derivatives: Quantum Chemistry Struggles to Predict the Enantioselectivity
}

\author{
Yuk Ping Chin and Elizabeth H. Krenske* \\ School of Chemistry and Molecular Biosciences, The University of Queensland, St Lucia, QLD \\ 4072, Australia \\ Email: e.krenske@uq.edu.au
}

\section{Supporting Information}

\section{Contents}

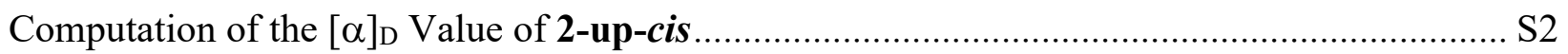

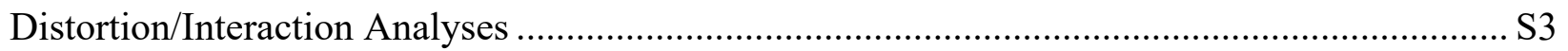

Boltzmann Analyses of Transition State Conformers ........................................................ S5

Supplementary 3D Diagrams of Important Intermediates and Transition States ..................... S6

Free energy diagram for the Ph-down stereoisomeric pathway in the Nazarov cyclization of 1

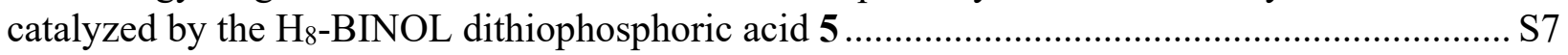

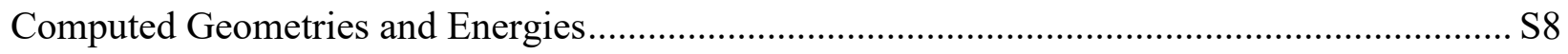

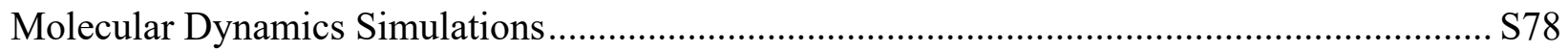

Computations of the Reactions Shown in Figure 8 of the Paper ............................................. S97

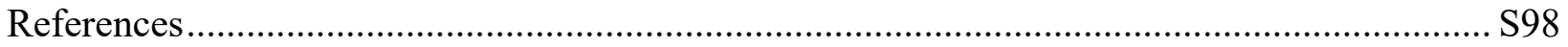




\section{Computation of the $[\alpha]_{D}$ Value of 2-up-cis}

The $[\alpha]_{D}$ value of the major enantiomer of 2-cis was reported by Rueping et al. ${ }^{1}$ to be -38 (c 0.1 , chloroform) at room temperature. Rueping et al. assigned this enantiomer as 2-up-cis. To explore this assignment, we computed the $[\alpha]_{\mathrm{D}}$ value of 2 -up-cis with three different DFT methods. In each case, the computational procedure began with a conformer search using B3LYP-D3(BJ)/6$31 \mathrm{G}(\mathrm{d})$ in the gas phase. Quasi-harmonically corrected free energies in chloroform were then computed at the M06-2X/6-311+G(d,p)-SMD//B3LYP-D3(BJ)/6-31G(d) level. Optical rotations in chloroform were computed at $589.28 \mathrm{~nm}$ at each of the three levels of theory ${ }^{2}$ listed in Table S1. Boltzmann averaging of the conformers' $[\alpha]_{D}$ values based on their M06-2X//B3LYP-D3(BJ) free energies gave the predicted $[\alpha]_{\mathrm{D}}$ values shown in the Table.

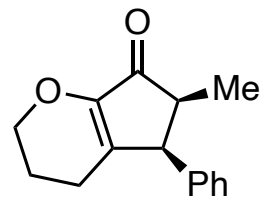

2-up-cis

Table S1. Computed $[\alpha]_{D}$ values of 2-up-cis

\begin{tabular}{|lc|}
\hline Method & {$[\boldsymbol{\alpha}]_{\mathbf{D}}$} \\
\hline B3LYP/aug-cc-pVDZ-PCM & -37.2 \\
B3LYP/aug-cc-pVTZ-PCM & -34.0 \\
B3LYP/aug-cc-pVTZ-SMD & -38.2 \\
\hline
\end{tabular}




\section{Distortion/Interaction Analyses}

Distortion/interaction analyses were performed on the transition states TS1-up/down and TS2up/down to explore the origins of the different selectivities predicted by different theoretical methods. For each TS, the distortion energies of the substrate $\Delta E_{\text {dist }}$ (sub) and catalyst $\Delta E_{\text {dist }}$ (cat) are calculated as shown in Figure $\mathrm{S} 1$. The interaction energy $\Delta E_{\text {int }}$ is obtained by subtracting the total distortion energy from the barrier height $\Delta E^{\ddagger}$. Tables S2 and S3 show how the values of $\Delta E^{+}$, $\Delta E_{\text {dist }}$ (sub), $\Delta E_{\text {dist }}$ (cat), $\Delta E_{\text {dist }}$ (total), and $\Delta E_{\text {int }}$ vary between the Ph-up and Ph-down transition state for each catalyst, at each of seven different levels of theory.

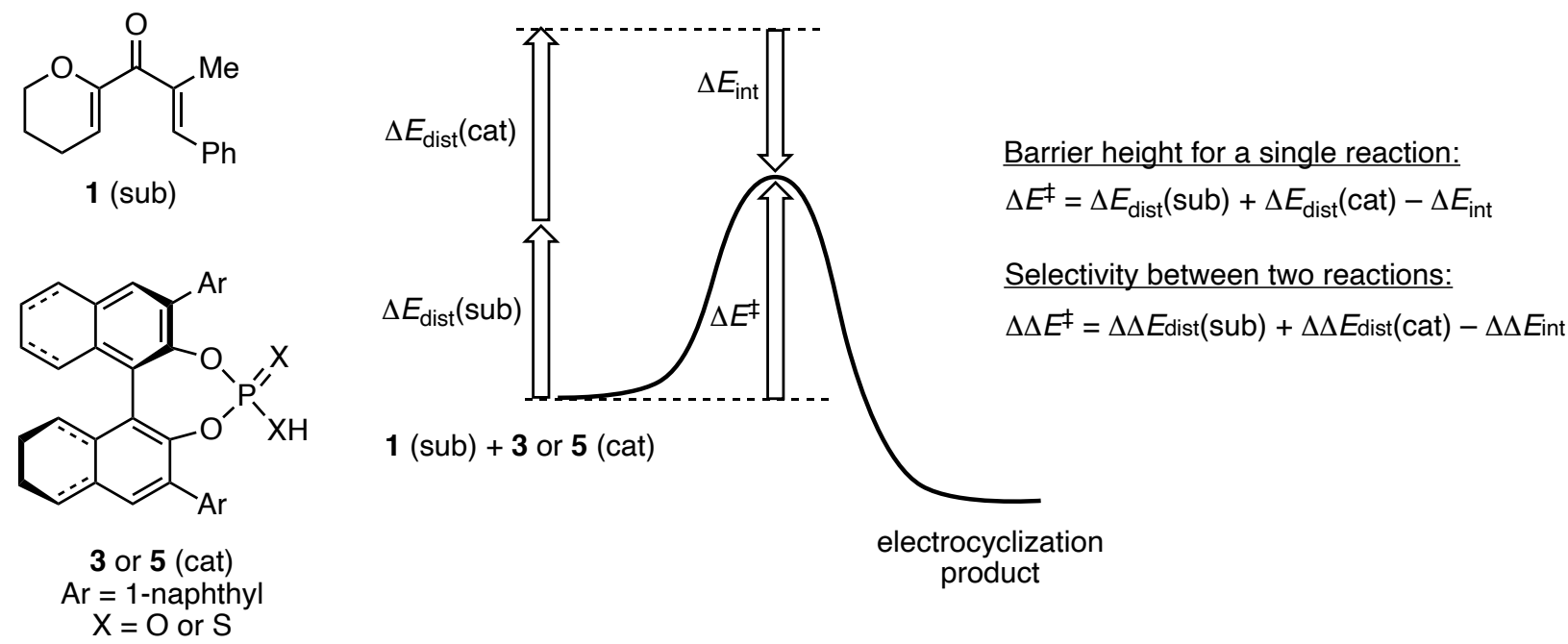

Figure S1. Distortion/interaction analysis of selectivities.

Table S2. Distortion/interaction analyses of the transition states TS1-up and TS1-down for the Nazarov cyclization of divinyl ketone $\mathbf{1}$ catalyzed by phosphoric acid $\mathbf{3}$.

\begin{tabular}{|lccccc|}
\hline Single point calculation method & \\
& $\Delta \Delta \boldsymbol{E}^{*}$ & $\begin{array}{c}\Delta \Delta \boldsymbol{E}_{\text {dist }} \\
\text { (sub) }\end{array}$ & $\begin{array}{c}\Delta \Delta \boldsymbol{E}_{\text {dist }} \\
\text { (cat) }\end{array}$ & $\begin{array}{c}\Delta \Delta \boldsymbol{E}_{\text {dist }} \\
\text { (total) }\end{array}$ & $\Delta \Delta \boldsymbol{E}_{\text {int }}$ \\
\hline M06-2X/6-311+G(d,p) & -0.8 & 3.6 & 24.6 & 28.1 & 28.9 \\
M06-2X/6-311+G(d,p)-PCM & -1.2 & 3.5 & 25.2 & 28.7 & 29.9 \\
M06-2X/def2-TZVPP & 0.2 & 3.6 & 24.6 & 28.2 & 28.0 \\
M06-2X-D3/6-311+G(d,p) & -1.1 & 3.2 & 17.1 & 20.3 & 21.3 \\
B3LYP-D3(BJ)/6-311+G(d,p) & -1.5 & 3.6 & 24.6 & 28.1 & 29.6 \\
B97-D/6-311+G(d,p) & -0.8 & 3.6 & 24.5 & 28.0 & 28.8 \\
wB97X-D/6-311+G(d,p) & -0.9 & 3.5 & 22.1 & 25.5 & 26.4 \\
\hline
\end{tabular}

${ }^{a}$ The single-point calculations were performed using geometries of TS1-up and TS1-down that were optimized with B3LYP-D3(BJ)/6-31G(d) in the gas phase. The single-point calculations used the SMD model of toluene unless otherwise stated. Values shown are the differences between TS1-down and TS1up. 
Table S3. Distortion/interaction analyses of the transition states TS2-up and TS2-down for the Nazarov cyclization of divinyl ketone $\mathbf{1}$ catalyzed by dithiophosphoric acid $\mathbf{5}$.

\begin{tabular}{|c|c|c|c|c|c|}
\hline Single point calculation method ${ }^{a}$ & $\Delta \Delta E^{*}$ & $\begin{array}{c}\Delta \Delta \boldsymbol{E}_{\text {dist }} \\
\text { (sub) }\end{array}$ & $\begin{array}{c}\Delta \Delta E_{\text {dist }} \\
\text { (cat) }\end{array}$ & $\begin{array}{c}\Delta \Delta E_{\text {dist }} \\
\text { (total) }\end{array}$ & $\Delta \Delta \boldsymbol{E}_{\mathrm{int}}$ \\
\hline M06-2X/6-311+G(d,p) & 0.5 & 3.2 & -2.5 & 0.7 & 0.2 \\
\hline M06-2X/6-311+G(d,p)-PCM & -1.7 & 3.2 & -4.4 & -1.2 & 0.5 \\
\hline M06-2X/def2-TZVPP & -1.3 & 3.3 & -4.9 & -1.7 & -0.3 \\
\hline M06-2X-D3/6-311+G(d,p) & 2.0 & 2.6 & -2.7 & -0.1 & -2.1 \\
\hline B3LYP-D3(BJ)/6-311+G(d,p) & 0.5 & 3.2 & -2.2 & 1.0 & 0.5 \\
\hline B97-D/6-311+G(d,p) & 0.8 & 3.2 & -2.8 & 0.4 & -0.5 \\
\hline$\omega B 97 X-D / 6-311+G(d, p)$ & -0.2 & 3.1 & -2.8 & 0.3 & 0.5 \\
\hline
\end{tabular}

${ }^{a}$ The single-point calculations were performed using geometries of TS2-up and TS2-down that were optimized with B3LYP-D3(BJ)/6-31G(d) in the gas phase. The single-point calculations used the SMD model of toluene unless otherwise stated. Values shown are the differences between TS2-down and TS2up.

The breaking O-H bond of the catalyst is $0.2 \AA$ longer in TS1-down (1.67 $\AA$ ) than it is in TS1-up (1.47 $\AA$ ). To explore how the longer O-H bond in TS1-down contributes to the catalyst distortion energy, the bond was manually shortened from $1.67 \AA$ to $1.47 \AA$ and the single-point energy of the catalyst fragment was recomputed. Table S4 lists the energy differences between the shortened and original catalyst fragments, which show that the bond lengthening contributes $19-26 \mathrm{kcal} / \mathrm{mol}$ toward the catalyst distortion energy in TS1-down.

Table S4. Difference between the energies of the catalyst fragment from TS1-down before and after shortening the $\mathrm{O}-\mathrm{H}$ bond from $1.67 \AA$ to $1.47 \AA$.

\begin{tabular}{|ll|}
\hline Single point calculation method $^{a}$ & Rel. $\boldsymbol{E}$ \\
\hline M06-2X/6-311+G(d,p) & -25.1 \\
M06-2X/6-311+G(d,p)-PCM & -25.5 \\
M06-2X/def2-TZVPP & -25.4 \\
M06-2X-D3/6-311+G(d,p) & -19.3 \\
B97-D/6-311+G(d,p) & -24.6 \\
wB97X-D/6-311+G(d,p) & -22.9 \\
B3LYP-D3(BJ)/6-311+G(d,p) & -25.1 \\
\hline
\end{tabular}

${ }^{a}$ The single-point calculations used the SMD model of toluene unless otherwise stated. 


\section{Boltzmann Analyses of Transition State Conformers}

Tables S5 and S6 show the energies and Boltzmann populations of TS conformers for the Nazarov cyclizations of 1 . The energies were calculated at the M06-2X/6-311+G(d,p)-SMD//B3LYP$\mathrm{D} 3(\mathrm{BJ}) / 6-31 \mathrm{G}(\mathrm{d})$ level of theory in toluene (for 3) or chloroform (for 5) as described in the manuscript. Populations were calculated at the experimental temperatures, viz. $60{ }^{\circ} \mathrm{C}$ for catalyst 3 and $20^{\circ} \mathrm{C}$ for catalyst 5 .

Table S5. Boltzmann analysis of the transition states for the Nazarov cyclization of $\mathbf{1}$ catalyzed by phosphoric acid 3.

\begin{tabular}{|lcc|}
\hline Transition State & $\begin{array}{c}\Delta \boldsymbol{G}^{\ddagger} \text { rel } \\
(\mathrm{kcal} / \mathrm{mol})\end{array}$ & $\mathbf{\%}$ \\
\hline Ph-up conformer \#1 (TS1-up) & 0.00 & 31.1 \\
Ph-up conformer \#2 & 0.51 & 14.4 \\
Ph-up conformer \#3 & 0.53 & 14.0 \\
Ph-up conformer \#4 & 0.62 & 12.2 \\
Ph-up conformer \#5 & 1.93 & 1.7 \\
Ph-up conformer \#6 & 2.42 & 0.8 \\
Ph-up conformer \#7 & 2.59 & 0.6 \\
Ph-up conformer \#8 & 4.91 & 0.02 \\
Ph-down conformer \#1 (TS1-down) & 0.41 & 16.7 \\
Ph-down conformer \#2 & 1.22 & 5.0 \\
Ph-down conformer \#3 & 1.62 & 2.7 \\
Ph-down conformer \#4 & 2.39 & 0.8 \\
\hline
\end{tabular}

Table S6. Boltzmann analysis of the transition states for the Nazarov cyclization of $\mathbf{1}$ catalyzed by dithiophosphoric acid $\mathbf{5}$.

\begin{tabular}{|lcc|}
\hline Transition State & $\begin{array}{c}\Delta \boldsymbol{G}^{\ddagger} \text { rel } \\
(\mathrm{kcal} / \mathrm{mol})\end{array}$ & $\mathbf{\%}$ \\
\hline Ph-up conformer \#1 (TS2-up) & 0.00 & 49.3 \\
Ph-up conformer \#2 & 3.17 & 0.2 \\
Ph-up conformer \#3 & 3.28 & 0.2 \\
Ph-down conformer \#1 (TS2-down) & 0.78 & 13.0 \\
Ph-down conformer \#2 & 0.83 & 12.0 \\
Ph-down conformer \#3 & 0.88 & 10.9 \\
Ph-down conformer \#4 & 1.01 & 8.7 \\
Ph-down conformer \#5 & 1.24 & 5.8 \\
\hline
\end{tabular}




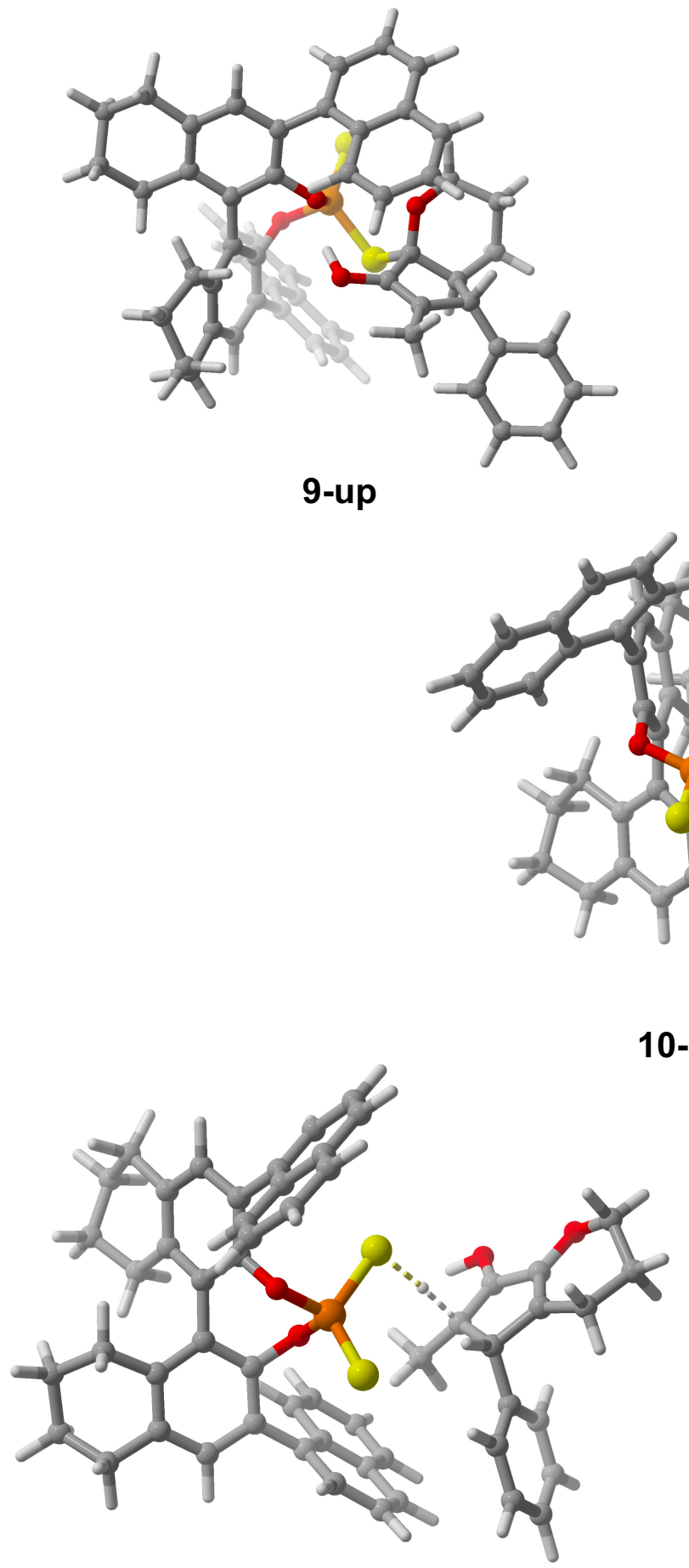

TS4

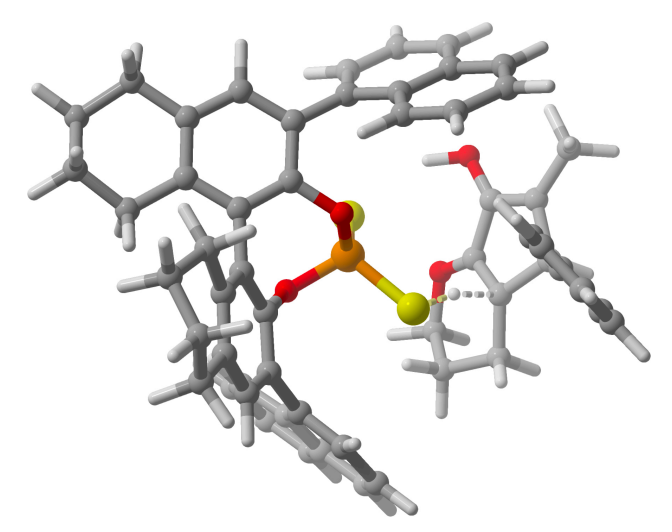

TS3

10-up

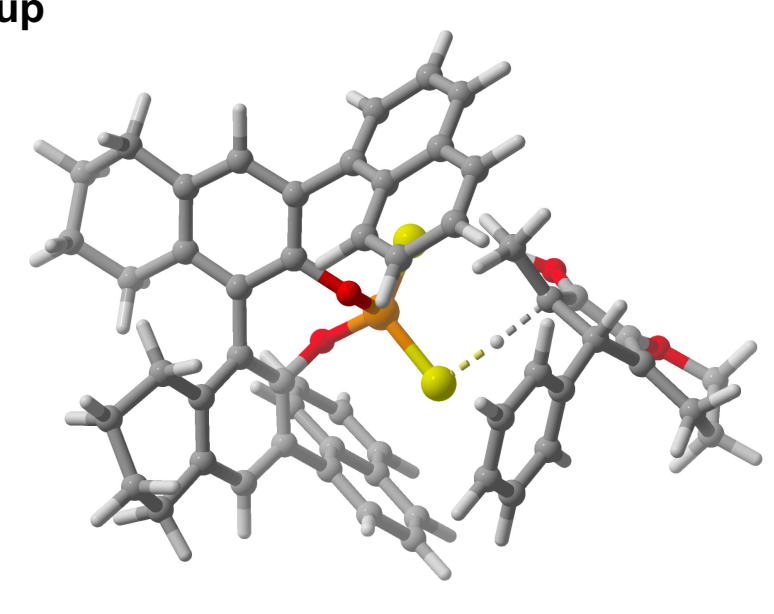

TS4'

Figure S2. 3D diagrams of important intermediates and transition states in the Nazarov cyclization of $\mathbf{1}$ catalyzed by the $\mathrm{H}_{8}$-BINOL dithiophosphoric acid $\mathbf{5}$. 
Free energy diagram for the Ph-down stereoisomeric pathway in the Nazarov cyclization of 1 catalyzed by the $\mathrm{H}_{8}$-BINOL dithiophosphoric acid 5

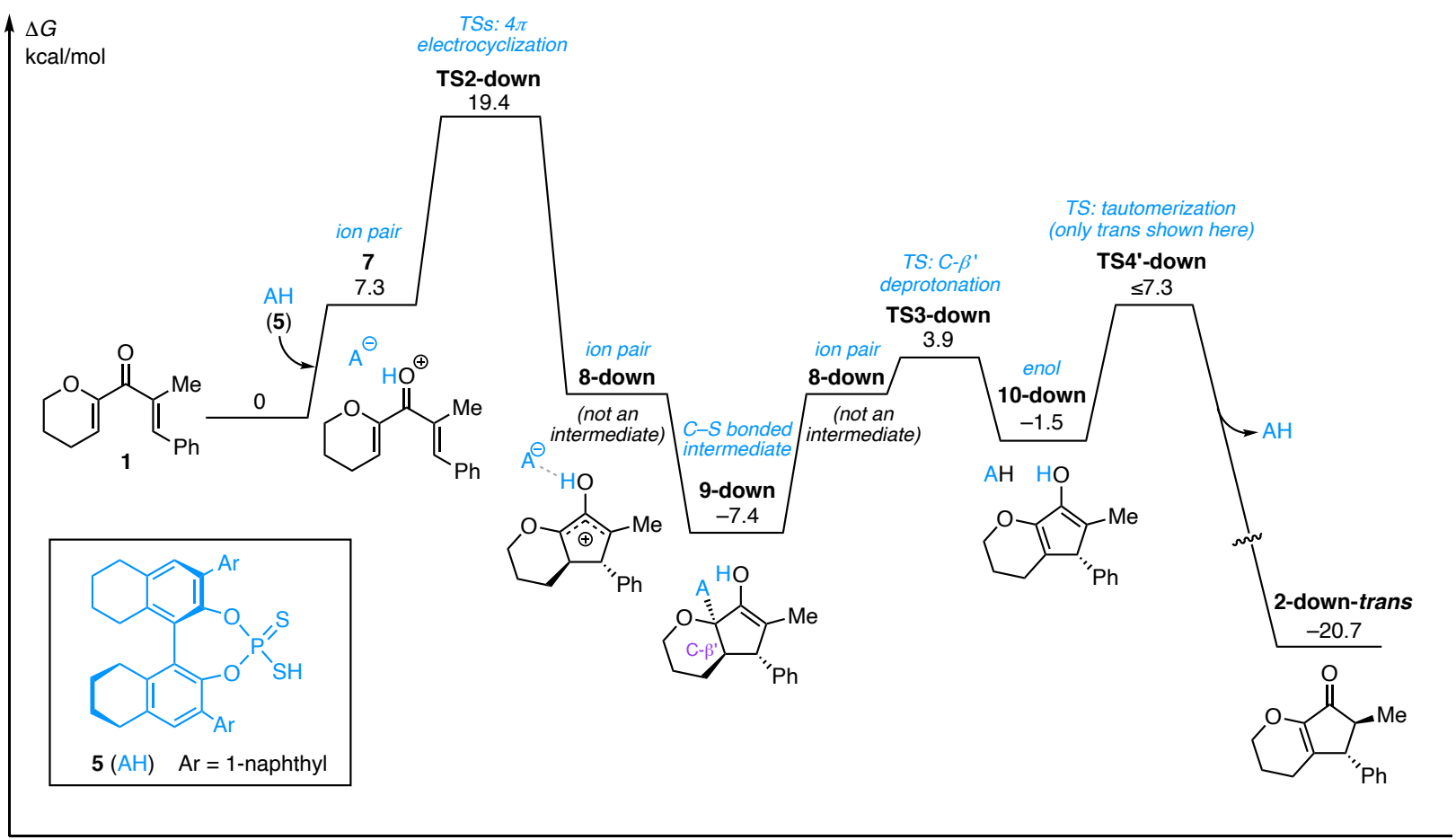

Figure S3. Free energy diagram for the Ph-down stereoisomeric pathway in the Nazarov cyclization of 1 catalyzed by the $\mathrm{H}_{8}$-BINOL dithiophosphoric acid $\mathbf{5}$ in chloroform, computed with M06-2X/6-311+G(d,p)-SMD//B3LYP-D3(BJ)/6-31G(d). Energies in $\mathrm{kcal} / \mathrm{mol}$. Only the formation of the trans product is shown, but both cis and trans isomers are obtained experimentally. 


\section{Computed Geometries and Energies}

Cartesian coordinates and energies of computed structures are listed below. Unless otherwise stated, the geometries were optimized with B3LYP-D3(BJ)/6-31G(d) in the gas phase. The reported energies are as follows:

$\mathrm{E}_{\text {toluene }} \quad$ Sum of M06-2X/6-311+G(d,p)-SMD single-point electronic energy and solvation energy in toluene

$\mathrm{G}_{\text {toluene }} \quad$ Total M06-2X/6-311+G(d,p)-SMD//B3LYP-D3(BJ)/6-31G(d) Gibbs free energy in toluene at $333.15 \mathrm{~K}$ and $1 \mathrm{~mol} / \mathrm{L}$ including quasiharmonic correction.

$\mathrm{E}_{\mathrm{chloroform}}$ Sum of M06-2X/6-311+G(d,p)-SMD single-point electronic energy and solvation energy in chloroform

$\mathrm{G}_{\text {chloroform }}$ Total M06-2X/6-311+G(d,p)-SMD//B3LYP-D3(BJ)/6-31G(d) Gibbs free energy at $293.15 \mathrm{~K}$ and $1 \mathrm{~mol} / \mathrm{L}$ including quasiharmonic correction.

1

$$
\text { 3. } 443729
$$

$$
2.229621
$$$$
\text { 1. } 287561
$$

$$
-0.187799
$$

$$
-0.767401
$$$$
1.763220
$$$$
-0.927221
$$$$
-0.116159
$$$$
-1.054296
$$$$
-1.919584
$$$$
-0.345822
$$$$
1.773357
$$

$$
2.791525
$$$$
\text { 3. } 700258
$$$$
2.253752
$$$$
\text { 3. } 378454
$$$$
4.402514
$$$$
4.423614
$$$$
4.806777
$$$$
5.270370
$$$$
\text { 3. } 710480
$$$$
-2.187384
$$$$
-2.615155
$$$$
-3.142826
$$$$
-3.947991
$$$$
-1.884986
$$$$
-4.474959
$$$$
-2.835662
$$$$
-4.886197
$$$$
-4.254970
$$$$
-5.194099
$$$$
-5.926841
$$$$
3.086706
$$

$$
0.370678
$$$$
0.346973
$$$$
1.509563
$$$$
1.359638
$$$$
0.146144
$$$$
2.598145
$$$$
2.657503
$$$$
-0.690059
$$$$
2.888875
$$$$
2.631007
$$

3.469404 $-0.689970$ $-1.596858$

$-2.021999$

$-2.439708$

$-1.102131$

$-0.782944$

$-2.765796$

$-0.998138$

$-0.512955$

1.264395

$-0.209506$

$-1.328628$

0.471536

$-1.726687$

$-1.879090$

0.064526

1.299070

$-1.027546$

$-2.585869$

0.599430

$-1.338922$

$-2.499276$

0 imaginary frequencies

$\mathrm{E}_{\text {toluene }}=-731.542239$

$\mathrm{E}_{\text {chloroform }}=-731.544838$

$\mathrm{G}_{\text {toluene }}=-731.303158$

$\mathrm{G}_{\text {chloroform }}=-731.306295$

$$
\begin{array}{r}
-0.591157 \\
-0.015554 \\
-0.182834 \\
0.004369 \\
-0.153309 \\
-0.495008 \\
0.204072 \\
-0.383742 \\
1.270206 \\
-0.253296 \\
-0.237410 \\
0.761649 \\
1.203351 \\
0.055498 \\
1.643438 \\
1.990706 \\
-0.517455 \\
0.407547 \\
-1.515265 \\
0.102981 \\
-1.143064 \\
-0.100522 \\
-0.840691 \\
0.676854 \\
-0.842506 \\
-1.428594 \\
0.685578 \\
1.304108 \\
-0.080126 \\
-1.432248 \\
1.299631 \\
-0.073549 \\
-0.717971
\end{array}
$$




\section{3}

P $\quad-0.196338$

O $\quad 0.243230$

O $\quad 0.893273$

$\mathrm{H} \quad-1.829428$

$\mathrm{H} \quad 0.361940$

C $\quad-1.735575$

$\mathrm{H} \quad-2.073329$

C $\quad-0.354569$

C $\quad-2.643018$

$\mathrm{H} \quad-3.705188$

$\mathrm{H} \quad 3.284814$

C $\quad 0.091242$

$\mathrm{H} \quad 1.151995$

C $\quad 3.562394$

$\mathrm{H} \quad 4.229952$

C $\quad-2.215901$

C $\quad-0.821352$

C $\quad 4.100102$

$\mathrm{H} \quad 0.164427$

C $\quad 3.344055$

$\mathrm{H} \quad 2.982108$

$\mathrm{H} \quad 3.934458$

C $\quad 3.389622$

C $\quad 1.213964$

C $\quad 1.975567$

C $\quad 3.917056$

C -3.141840

H $\quad-4.195715$

C $\quad 1.781422$

C $\quad 3.156783$

C $\quad-0.399536$

C $\quad 4.460752$

C $\quad 1.023772$

C $\quad 3.729656$

C $\quad 3.739974$

$\mathrm{H} \quad 4.880656$

$\mathrm{H} \quad 4.962321$

$\mathrm{H} \quad 4.775039$

$\mathrm{H} \quad 1.519093$

C $\quad 1.633279$

C $\quad 2.991888$

C $\quad-2.734257$

C $\quad 4.296756$

C $\quad-1.352246$

C $\quad 3.576417$

O -1.153982

$\mathrm{H} \quad-3.449685$

C $\quad-3.690528$

C $\quad-3.953901$

C $\quad 4.676922$

$\mathrm{H} \quad-3.331897$

O $\quad-0.950197$

C $\quad 3.967952$

C $\quad-4.054329$

H $\quad 5.093015$

C $\quad-4.326082$

C $\quad 4.520035$

$$
\begin{array}{rr}
-1.425880 & -0.872550 \\
-2.291949 & -1.976312 \\
-0.801999 & 0.152163 \\
-2.644710 & -0.139258 \\
4.837321 & 3.396977 \\
4.363502 & 3.117713 \\
5.052444 & 3.886305 \\
4.245636 & 2.834638 \\
3.592656 & 2.430421 \\
3.657345 & 2.651746 \\
-1.612524 & 4.063088 \\
3.383482 & 1.858790 \\
3.296061 & 1.657922 \\
-2.075714 & 3.120502 \\
-3.918809 & 4.051205 \\
2.682758 & 1.427193 \\
2.592998 & 1.109791 \\
-3.384285 & 3.114434 \\
4.091688 & -0.842481 \\
4.777290 & -1.854633 \\
-0.381684 & 1.957540 \\
5.604078 & -2.238482 \\
-1.386707 & 1.941953 \\
3.934195 & -1.059017 \\
4.968911 & -1.553259 \\
3.540158 & -1.676402 \\
1.842298 & 0.760096 \\
1.912817 & 1.014614 \\
2.651073 & -0.836254 \\
2.448120 & -1.180962 \\
1.680710 & 0.086398 \\
-3.973628 & 1.925219 \\
1.545047 & -0.324044 \\
1.158808 & -1.044864 \\
-1.970059 & 0.695968 \\
-4.976363 & 1.910442 \\
3.372924 & -1.922866 \\
1.015704 & -1.303004 \\
5.940419 & -1.719231 \\
0.303865 & -0.271758 \\
0.077626 & -0.612750 \\
0.917599 & -0.177242 \\
-3.289951 & 0.691783 \\
0.860552 & -0.490814 \\
-1.288021 & -0.551374 \\
-2.064039 & 0.249406 \\
0.916849 & -2.748564 \\
-0.009061 & -0.843430 \\
0.133015 & -2.192042 \\
-3.882164 & -0.540966 \\
-0.680494 & 1.782538 \\
-0.091848 & -1.427231 \\
-1.900189 & -1.725665 \\
-1.305106 & 1.270686 \\
-4.886408 & -0.530796 \\
-1.057676 & -0.103721 \\
-3.201009 & -1.723837
\end{array}
$$




$\begin{array}{lrlr}\mathrm{C} & -4.863655 & -0.718971 & -2.855825 \\ \mathrm{H} & 3.819457 & -1.380010 & -2.666792 \\ \mathrm{H} & -5.052345 & -0.576362 & -3.915646 \\ \mathrm{C} & -4.675118 & -2.335123 & 1.940306 \\ \mathrm{H} & -4.447090 & -2.511877 & 2.987430 \\ \mathrm{C} & -5.250920 & -1.919648 & -0.782724 \\ \mathrm{C} & -5.504224 & -1.719808 & -2.164174 \\ \mathrm{H} & 4.806251 & -3.660471 & -2.665220 \\ \mathrm{C} & -5.603831 & -3.170376 & 1.274509 \\ \mathrm{C} & -5.880965 & -2.965607 & -0.056770 \\ \mathrm{H} & -6.207143 & -2.378977 & -2.666997 \\ \mathrm{H} & -6.089345 & -3.976927 & 1.816300 \\ \mathrm{H} & -6.584463 & -3.608343 & -0.579975 \\ 0 & \text { imaginary frequencies } & \\ \mathrm{E}_{\text {toluene }}=-2181.486252 & \\ \mathrm{G}_{\text {toluene }}=-2180.999804 & \end{array}$

\section{TS1-up}

$\begin{array}{lrrr}\text { C } & 6.775704 & -3.228923 & -0.747761 \\ \mathrm{C} & 6.882985 & -1.818743 & -0.706869 \\ \mathrm{C} & 5.799253 & -1.042347 & -0.364523 \\ \mathrm{C} & 4.548013 & -1.633247 & -0.040019 \\ \mathrm{C} & 4.431905 & -3.059648 & -0.117903 \\ \mathrm{C} & 5.572649 & -3.831325 & -0.463619 \\ \mathrm{C} & 3.395272 & -0.860978 & 0.314880 \\ \mathrm{C} & 2.185277 & -1.516338 & 0.491979 \\ \mathrm{C} & 2.055881 & -2.928630 & 0.424383 \\ \mathrm{C} & 3.178857 & -3.672722 & 0.133419 \\ \mathrm{C} & 3.425779 & 0.620971 & 0.449666 \\ \mathrm{C} & 4.265354 & 1.290977 & 1.397995 \\ \mathrm{C} & 4.211695 & 2.720682 & 1.484802 \\ \mathrm{C} & 3.301010 & 3.435202 & 0.668265 \\ \mathrm{C} & 2.454598 & 2.789733 & -0.208626 \\ \mathrm{C} & 2.540540 & 1.375329 & -0.306059 \\ \mathrm{C} & 5.122555 & 0.597298 & 2.295070 \\ \mathrm{C} & 5.913984 & 1.279409 & 3.191100 \\ \mathrm{C} & 5.889383 & 2.692989 & 3.246043 \\ \mathrm{C} & 5.049735 & 3.393863 & 2.412932 \\ \mathrm{O} & 1.049903 & -0.777971 & 0.749401 \\ \mathrm{O} & 1.694964 & 0.734087 & -1.183892 \\ \mathrm{P} & 0.347212 & 0.008419 & -0.536216 \\ \mathrm{O} & -0.061823 & -0.984478 & -1.599343 \\ \mathrm{O} & -0.595216 & 1.009658 & 0.046947 \\ \mathrm{H} & 7.639947 & -3.830142 & -1.015349 \\ \mathrm{H} & 7.828256 & -1.343846 & -0.954360 \\ \mathrm{H} & 5.889463 & 0.037325 & -0.346918 \\ \mathrm{H} & 5.470569 & -4.912783 & -0.508993 \\ \mathrm{H} & 3.102117 & -4.755443 & 0.079635 \\ \mathrm{H} & 3.257577 & 4.517828 & 0.752673 \\ \mathrm{H} & 5.143400 & -0.485350 & 2.270022 \\ \mathrm{H} & 6.559953 & 0.726201 & 3.867096 \\ \mathrm{H} & 6.523345 & 3.218714 & 3.954336 \\ \mathrm{H} & 5.004389 & 4.479246 & 2.457446 \\ \mathrm{H} & -1.373557 & -1.625941 & -1.776754 \\ \mathrm{C} & 1.479704 & 3.551910 & -1.035133 \\ \mathrm{C} & 1.622960 & 3.590499 & -2.408989 \\ \mathrm{C} & 0.772709 & 4.381194 & -3.213141 \\ \mathrm{C} & -0.218789 & 5.141672 & -2.636926\end{array}$




\begin{tabular}{|c|c|c|c|}
\hline & -0.429627 & 5.104702 & -1.234366 \\
\hline & -1.474808 & 5.846353 & -0.619467 \\
\hline & -1.711012 & 5.748721 & 0.732074 \\
\hline & -0.911389 & 4.894667 & 1.530244 \\
\hline & 0.123185 & 4.180724 & 0.970011 \\
\hline & 0.411219 & 4.278152 & -0.418427 \\
\hline & -2.090926 & 6.489983 & -1.243413 \\
\hline & -2.516002 & 6.318035 & 1.188695 \\
\hline & -1.121371 & 4.798171 & 2.591634 \\
\hline & 0.716257 & 3.508659 & 1.577932 \\
\hline & -0.860796 & 5.772428 & -3.247302 \\
\hline & 0.921230 & 4.398483 & -4.289113 \\
\hline & 2.414548 & 3.011067 & -2.872697 \\
\hline & 0.741879 & -3.576631 & 0.695666 \\
\hline & 0.251960 & -3.594593 & 1.987280 \\
\hline & -0.952509 & -4.260488 & 2.304427 \\
\hline & -1.663508 & -4.909507 & 1.321139 \\
\hline & -1.218593 & -4.887149 & -0.025595 \\
\hline & -1.956628 & -5.516397 & -1.062450 \\
\hline & -1.533382 & -5.451563 & -2.368780 \\
\hline & -0.348606 & -4.750375 & -2.695976 \\
\hline & 0.399577 & -4.143942 & -1.713874 \\
\hline & -0.003353 & -4.200973 & -0.352792 \\
\hline & 0.814123 & -3.097200 & 2.771615 \\
\hline & -1.302005 & -4.271730 & 3.333267 \\
\hline & -2.579696 & -5.444843 & 1.559998 \\
\hline & -2.870111 & -6.046302 & -0.801778 \\
\hline & -2.111468 & -5.929523 & -3.154845 \\
\hline & -0.033989 & -4.681916 & -3.733323 \\
\hline & 1.289580 & -3.582771 & -1.970942 \\
\hline & -3.416475 & 1.081351 & -0.233143 \\
\hline & -2.942310 & 0.286703 & -1.283353 \\
\hline & -3.131986 & -1.140359 & -1.170633 \\
\hline & -4.243745 & -1.467885 & -0.392618 \\
\hline & -5.141067 & -0.383671 & -0.145850 \\
\hline ) & -2.334975 & -2.027272 & -1.711772 \\
\hline & -4.400835 & -2.862979 & 0.144640 \\
\hline & -5.502424 & 0.185136 & -0.995334 \\
\hline H & -5.390720 & -3.028576 & 0.574423 \\
\hline 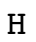 & -4.235138 & -3.574745 & -0.671208 \\
\hline 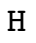 & -3.637017 & -3.090434 & 0.897543 \\
\hline ) & -2.442589 & 0.762680 & -2.414382 \\
\hline 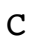 & -2.110370 & 2.181756 & -2.426712 \\
\hline 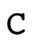 & -3.273438 & 2.980745 & -1.856101 \\
\hline H & -1.203636 & 2.305768 & -1.833057 \\
\hline $\mathrm{f}$ & -1.910672 & 2.404082 & -3.474228 \\
\hline c & -3.493405 & 2.568008 & -0.394615 \\
\hline $\mathrm{H}$ & -4.171562 & 2.788477 & -2.457468 \\
\hline $\mathrm{H}$ & -2.709283 & 3.004788 & 0.237145 \\
\hline $\mathrm{H}$ & -4.451033 & 2.942480 & -0.007998 \\
\hline $\mathrm{H}$ & -3.293624 & 0.703605 & 0.769848 \\
\hline C & -5.973626 & -0.260961 & 1.047508 \\
\hline C & -7.186151 & 0.448538 & 0.978619 \\
\hline $\mathrm{C}$ & -5.567553 & -0.784334 & 2.289808 \\
\hline C & -7.985817 & 0.603072 & 2.106800 \\
\hline $\mathrm{H}$ & -7.499240 & 0.870600 & 0.026936 \\
\hline C & -6.365384 & -0.625760 & 3.417629 \\
\hline 4 & -4.615198 & -1.298980 & 2.367139 \\
\hline
\end{tabular}




$\begin{array}{lrrr}\mathrm{C} & -7.578158 & 0.063531 & 3.328732 \\ \mathrm{H} & -8.923759 & 1.145792 & 2.036065 \\ \mathrm{H} & -6.039186 & -1.031907 & 4.370435 \\ \mathrm{H} & -8.198420 & 0.187492 & 4.211575 \\ \mathrm{H} & -3.050800 & 4.048438 & -1.917550 \\ 1 & \text { imaginary frequency } & \\ \mathrm{E}_{\text {toluene }}=-2913.023966 & \\ \mathrm{G}_{\text {toluene }}=-2912.272982 & \end{array}$

\section{TS1-down}

\begin{tabular}{|c|c|c|c|}
\hline C & -2.424051 & -5.209797 & 3.255246 \\
\hline C & -3.376477 & -4.173677 & 3.111552 \\
\hline $\mathrm{C}$ & -3.239665 & -3.226308 & 2.122028 \\
\hline C & -2.142760 & -3.263071 & 1.219594 \\
\hline C & -1.156434 & -4.286601 & 1.395546 \\
\hline C & -1.335887 & -5.258016 & 2.415987 \\
\hline C & -1.962307 & -2.304624 & 0.168555 \\
\hline C & -0.793742 & -2.339940 & -0.572831 \\
\hline $\mathrm{C}$ & 0.219041 & -3.321654 & -0.381930 \\
\hline C & -0.003742 & -4.293603 & 0.57269 \\
\hline C & -2.967751 & -1.244880 & -0.11426 \\
\hline C & -4.323913 & -1.576080 & -0.44127 \\
\hline C & -5.273945 & -0.521080 & -0.617726 \\
\hline C & -4.844001 & 0.821129 & -0.497287 \\
\hline C & -3.524464 & 1.151426 & -0.258119 \\
\hline $\mathrm{C}$ & -2.585573 & 0.091656 & -0.09967 \\
\hline C & -4.765547 & -2.913411 & -0.63446 \\
\hline C & -6.078241 & -3.189096 & -0.94319 \\
\hline C & -7.023873 & -2.145174 & -1.07704 \\
\hline C & -6.624176 & -0.838998 & -0.92160 \\
\hline $\mathrm{O}$ & -0.604024 & -1.373480 & -1.53828 \\
\hline $\mathrm{O}$ & -1.272277 & 0.425554 & \\
\hline $\mathrm{P}$ & -0.084609 & 0.103442 & -0.994258 \\
\hline $\mathrm{O}$ & 1.187769 & -0.025168 & -0.21606 \\
\hline $\mathrm{O}$ & -0.234859 & 1.042064 & -2.167402 \\
\hline $\mathrm{H}$ & -2.548201 & -5.955345 & 4.03543 \\
\hline $\mathrm{H}$ & -4.222317 & -4.122489 & 3.79138 \\
\hline $\mathrm{H}$ & -3.972481 & -2.434276 & 2.02689 \\
\hline $\mathrm{H}$ & -0.584316 & -6.035735 & 2.52722 \\
\hline $\mathrm{H}$ & 0.736230 & -5.075300 & 0.71932 \\
\hline $\mathrm{H}$ & -5.576903 & 1.616394 & -0.60055 \\
\hline $\mathrm{H}$ & -4.050079 & -3.721692 & -0.54582 \\
\hline $\mathrm{H}$ & -6.390018 & -4.219089 & -1.09170 \\
\hline $\mathrm{H}$ & -8.057850 & -2.378002 & -1.31477 \\
\hline $\mathrm{H}$ & -7.333798 & -0.023903 & -1.04012 \\
\hline C & -3.155935 & 2.592326 & -0.19133 \\
\hline C & -3.504348 & 3.403130 & -1.25835 \\
\hline C & -3.326877 & 4.803735 & -1.21775 \\
\hline $\mathrm{C}$ & -2.793021 & 5.399383 & -0.10013 \\
\hline C & -2.399309 & 4.613270 & 1.01443 \\
\hline C & -1.838561 & 5.214302 & 2.17351 \\
\hline $\mathrm{C}$ & -1.463800 & 4.452621 & 3.25555 \\
\hline C & -1.650033 & 3.050022 & 3.22750 \\
\hline C & -2.198538 & 2.437671 & 2.12343 \\
\hline C & -2.575347 & 3.189256 & 0.97761 \\
\hline $\mathrm{H}$ & -1.715801 & 6.294794 & 2.18884 \\
\hline $\mathrm{H}$ & -1.033379 & 4.924074 & 4.13455 \\
\hline $\mathrm{H}$ & -1.361831 & 2.451540 & 4.08673 \\
\hline
\end{tabular}




\section{TS1-up, reoptimized in SMD implicit toluene}

\begin{tabular}{|c|c|c|c|}
\hline & 6.767386 & -3.257615 & -0.848185 \\
\hline & 6.881777 & -1.848192 & -0.800491 \\
\hline & 5.808298 & -1.068921 & -0.431838 \\
\hline & 4.560224 & -1.655683 & -0.087326 \\
\hline & 4.436657 & -3.081444 & -0.171840 \\
\hline & 5.567052 & -3.856571 & -0.544563 \\
\hline & 3.417032 & -0.881114 & 0.296401 \\
\hline & 2.207869 & -1.533779 & 0.490202 \\
\hline & 2.072067 & -2.946413 & 0.419298 \\
\hline & 3.187002 & -3.692399 & 0.101857 \\
\hline & 3.459114 & 0.600079 & 0.447269 \\
\hline C & 4.326500 & 1.251695 & 1.384798 \\
\hline$C$ & 4.299190 & 2.681910 & 1.478432 \\
\hline & 3.390111 & 3.416472 & 0.677517 \\
\hline & 2.515765 & 2.790174 & -0.185835 \\
\hline & 2.567926 & 1.372949 & -0.283975 \\
\hline$C$ & 5.188917 & 0.540362 & 2.263326 \\
\hline$C$ & 6.007037 & 1.205273 & 3.148787 \\
\hline & 6.006658 & 2.618793 & 3.210605 \\
\hline & 5.164330 & 3.337361 & 2.394925 \\
\hline $\mathrm{O}$ & 1.081877 & -0.791780 & 0.771451 \\
\hline 0 & 1.695559 & 0.751769 & -1.148805 \\
\hline $\mathrm{P}$ & 0.357287 & 0.006701 & -0.497775 \\
\hline 0 & -0.048383 & -0.976276 & -1.571844 \\
\hline 0 & -0.591983 & 0.982392 & 0.113493 \\
\hline 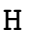 & 7.623704 & -3.860823 & -1.136320 \\
\hline $\mathrm{H}$ & 7.824178 & -1.375660 & -1.063312 \\
\hline $\mathrm{H}$ & 5.906046 & 0.009971 & -0.410660 \\
\hline & 5.458908 & -4.937178 & -0.594628 \\
\hline $\mathrm{H}$ & 3.108555 & -4.774714 & 0.044952 \\
\hline $\mathrm{H}$ & 3.373595 & 4.499606 & 0.762996 \\
\hline $\mathrm{H}$ & 5.192381 & -0.542426 & 2.235920 \\
\hline $\mathrm{H}$ & 6.655230 & 0.638071 & 3.811044 \\
\hline $\mathrm{H}$ & 6.661320 & 3.130533 & 3.910366 \\
\hline $\mathrm{H}$ & 5.137597 & 4.423101 & 2.444611 \\
\hline $\mathrm{H}$ & -1.400104 & -1.571156 & -1.740299 \\
\hline C & 1.548260 & 3.573855 & -1.002866 \\
\hline C & 1.683593 & 3.611325 & -2.377859 \\
\hline C & 0.834361 & 4.406896 & -3.178347 \\
\hline C & -0.152777 & 5.169557 & -2.597733 \\
\hline C & -0.353593 & 5.137597 & -1.193367 \\
\hline C & -1.395431 & 5.882074 & -0.575918 \\
\hline$C$ & -1.617882 & 5.798027 & 0.779185 \\
\hline C & -0.805506 & 4.957357 & 1.578601 \\
\hline C & 0.223048 & 4.237162 & 1.014719 \\
\hline C & 0.493339 & 4.314491 & -0.378739 \\
\hline 1 & -2.018601 & 6.518095 & -1.200321 \\
\hline $\mathrm{H}$ & -2.419809 & 6.369668 & 1.238363 \\
\hline $\mathrm{H}$ & -1.000448 & 4.876231 & 2.644243 \\
\hline $\mathrm{H}$ & 0.827442 & 3.580269 & 1.628528 \\
\hline $\mathrm{H}$ & -0.798792 & 5.798513 & -3.205542 \\
\hline $\mathrm{H}$ & 0.977437 & 4.421580 & -4.255116 \\
\hline $\mathrm{H}$ & 2.468462 & 3.027464 & -2.847982 \\
\hline C & 0.761329 & -3.591133 & 0.715568 \\
\hline C & 0.280004 & -3.579441 & 2.011222 \\
\hline & -0.924085 & -4.233850 & 2.352654 \\
\hline & -1.644878 & -4.903345 & 1.390297 \\
\hline
\end{tabular}




\begin{tabular}{|c|c|c|c|}
\hline C & -1.206627 & -4.917375 & 0.040904 \\
\hline C & -1.952825 & -5.573131 & -0.974313 \\
\hline C & -1.537931 & -5.544537 & -2.285004 \\
\hline & -0.355412 & -4.852149 & -2.638890 \\
\hline & 0.398652 & -4.218766 & -1.678151 \\
\hline & 0.007510 & -4.240774 & -0.312273 \\
\hline $\mathrm{H}$ & 0.848012 & -3.067270 & 2.781909 \\
\hline & -1.267002 & -4.216974 & 3.383563 \\
\hline & -2.562399 & -5.427232 & 1.647700 \\
\hline & -2.865196 & -6.094192 & -0.693 \\
\hline & -2.120665 & -6.044268 & -3.054 \\
\hline & -0.046865 & -4.812663 & -3.679762 \\
\hline & 1.288802 & -3.668487 & -1.957805 \\
\hline & -3.5 & & -0 . \\
\hline & -2.987802 & 0.344024 & -1.251 \\
\hline & -3.173765 & -1.082894 & -1.159363 \\
\hline & -4.306414 & -1.428112 & -0.422457 \\
\hline & -5.2 & -0 & -0 . \\
\hline 0 & -2.360660 & -1.96 & -1.69 \\
\hline & -4.474001 & -2.830317 & 0.091343 \\
\hline & -5.560530 & 0.215168 & -1.054422 \\
\hline & -5.4 & -3 & 705 \\
\hline $\mathrm{H}$ & -4.299864 & -3. & -0.7 \\
\hline $\mathrm{H}$ & -3.726534 & -3.067192 & 0.857699 \\
\hline c & -2.437656 & 0.83 & -2.355726 \\
\hline & -2.1 & 2.2 & -2 . \\
\hline C & -3.328507 & 3.036726 & -1.83 \\
\hline $\mathrm{H}$ & -1.255080 & 2.382638 & -1.689465 \\
\hline $\mathrm{H}$ & -1.860751 & 2.48 & -3.370251 \\
\hline & -3.6 & 2.6 & -0.3 \\
\hline $\mathrm{H}$ & -4.183275 & 2.839223 & -2.497982 \\
\hline $\mathrm{H}$ & -2.927654 & 3.084729 & 0.293334 \\
\hline $\mathrm{H}$ & -4.637932 & 2.950760 & -0.084822 \\
\hline $\mathrm{H}$ & -3 & 2 & \\
\hline C & -6.099668 & -0.24 & 0.966818 \\
\hline C & -7.311974 & 0.458341 & 0.853014 \\
\hline C & -5.742626 & -0.782012 & 2.219696 \\
\hline C & & & \\
\hline $\mathrm{H}$ & -7.5862 & 0.8883 & -0.106933 \\
\hline C & -6.588610 & -0.638588 & 3.314084 \\
\hline $\mathrm{H}$ & -4.792337 & -1.293541 & 2.333902 \\
\hline C & & 0.045997 & 3.180416 \\
\hline $\mathrm{H}$ & -9.096547 & 1.135347 & 1.843517 \\
\hline $\mathrm{H}$ & -6.301392 & -1.053344 & 4.275753 \\
\hline $\mathrm{H}$ & -8.458219 & 0.157039 & 4.037438 \\
\hline $\mathrm{H}$ & -3.113790 & 4.107582 & -1.882045 \\
\hline \multicolumn{4}{|c|}{ imaginary frequency } \\
\hline \multirow{2}{*}{\multicolumn{4}{|c|}{$E_{\text {toluene }}=-2913}}$. \\
\hline & & & \\
\hline
\end{tabular}

\section{TS1-down, reoptimized in SMD implicit toluene}

$\begin{array}{llll}\text { C } & -2.463174 & -5.024134 & 3.435740 \\ \text { C } & -3.399130 & -3.977335 & 3.263400 \\ \text { C } & -3.262659 & -3.076013 & 2.231265 \\ \text { C } & -2.182069 & -3.171424 & 1.313212 \\ \text { C } & -1.212995 & -4.207442 & 1.514751 \\ \text { C } & -1.391536 & -5.130081 & 2.580114\end{array}$




\begin{tabular}{|c|c|c|c|}
\hline & -2.000269 & -2.258914 & 0.221513 \\
\hline C & -0.845271 & -2.349900 & -0.536476 \\
\hline C & 0.139868 & -3.357942 & -0.336620 \\
\hline & -0.080859 & -4.280561 & 0.665851 \\
\hline & -2.991090 & -1.192950 & -0.092861 \\
\hline & -4.355436 & -1.515700 & -0.397911 \\
\hline & -5.290388 & -0.453330 & -0.610919 \\
\hline & -4.840564 & 0.886524 & -0.541421 \\
\hline & -3.515545 & 1.204911 & -0.319489 \\
\hline & -2.589327 & 0.137543 & -0.133415 \\
\hline & -4.820651 & -2.852444 & -0.533477 \\
\hline & -6.139863 & -3.119058 & -0.823734 \\
\hline & -7.069242 & -2.066165 & -0.995486 \\
\hline & -6.647478 & -0.761110 & -0.895437 \\
\hline c & -0.640644 & -1.414135 & -1.528587 \\
\hline & -1.270313 & 0.463423 & 0.076286 \\
\hline & -0.077269 & 0.062443 & -1.021411 \\
\hline 0 & 1.176794 & -0.0 & -0.21 \\
\hline c & -0.175336 & 0.958400 & -2.234509 \\
\hline & -2.586900 & -5.732661 & 4.249878 \\
\hline & -4.231703 & -3.880882 & 3.954731 \\
\hline $\mathrm{H}$ & -3.982554 & -2.2 & 6675 \\
\hline & -0.653374 & -5.917508 & 2.711175 \\
\hline $\mathrm{H}$ & 0.640062 & -5.076968 & 0.827151 \\
\hline & -5.563199 & 1.687555 & -0.668745 \\
\hline $\mathrm{H}$ & -4.1 & -3. & -0.4 \\
\hline $\mathrm{H}$ & -6.469002 & -4.149168 & -0.928089 \\
\hline $\mathrm{H}$ & -8.108285 & -2.291630 & -1.218175 \\
\hline F & -7.344459 & 0116 & -1.04 \\
\hline C & -3.122882 & 2.6 & $-0.2 \varepsilon$ \\
\hline C & -3.427061 & 3.428275 & -1.387190 \\
\hline C & -3.210962 & 4.824440 & -1.383363 \\
\hline C & -2.684763 & 5.438686 & -0.271983 \\
\hline & -2.3 & 4.6 & 0.8 \\
\hline C & -1.790863 & 5.296174 & 2.030272 \\
\hline C & -1.466598 & 4.557040 & 3.144278 \\
\hline C & -1.692396 & 3.159924 & 3.153175 \\
\hline & -2.2 & & \\
\hline C & -2.556341 & 3.257282 & 0.876551 \\
\hline $\mathrm{H}$ & -1.636110 & 6.372413 & 2.016555 \\
\hline $\mathrm{H}$ & -1.04491 & 5.0423 & 4.020072 \\
\hline $\mathrm{H}$ & -1.4440 & $2.5 \varepsilon$ & 4.037504 \\
\hline $\mathrm{H}$ & -2.40 & 1.461896 & 2.079759 \\
\hline $\mathrm{H}$ & -2.522450 & 6.513586 & -0.254419 \\
\hline $\mathrm{H}$ & -3.465413 & 5.405567 & -2.265163 \\
\hline $\mathrm{H}$ & -3.842288 & 2.9550 & -2.271353 \\
\hline C & 1.34 & -3.4205 & -1.199533 \\
\hline C & 1.201575 & -3.506339 & -2.572587 \\
\hline C & 2.316639 & -3.630688 & -3.428082 \\
\hline C & 3.588344 & -3.674544 & -2.907033 \\
\hline C & 3.795752 & -3.563193 & -1.508525 \\
\hline C & 5.105756 & -3.562015 & -0.957586 \\
\hline C & 5.306953 & -3.401987 & 0.393130 \\
\hline C & 4.197354 & -3.224525 & 1.252972 \\
\hline C & 2.914582 & -3.225374 & 0.753221 \\
\hline C & 2.666468 & -3.415392 & -0.634326 \\
\hline $\mathrm{H}$ & 0.204475 & -3.486633 & -2.998993 \\
\hline $\mathrm{H}$ & 2.159452 & -3.700387 & -4.500567 \\
\hline
\end{tabular}




\begin{tabular}{|c|c|c|c|}
\hline $\mathrm{H}$ & 4.452751 & -3.780893 & -3.557597 \\
\hline $\mathrm{H}$ & 5.950112 & -3.680145 & -1.632387 \\
\hline $\mathrm{H}$ & 6.313676 & -3.398856 & 0.801547 \\
\hline $\mathrm{H}$ & 4.362150 & -3.077742 & 2.316193 \\
\hline $\mathrm{H}$ & 2.075489 & -3.059053 & 1.418054 \\
\hline $\mathrm{C}$ & 1.926804 & 2.945181 & -0.077946 \\
\hline $\mathrm{C}$ & 1.899987 & 2.863583 & -1.473097 \\
\hline $\mathrm{C}$ & 2.620027 & 1.783089 & -2.085208 \\
\hline $\mathrm{C}$ & 3.664408 & 1.301502 & -1.309239 \\
\hline $\mathrm{C}$ & 4.035096 & 2.147745 & -0.218849 \\
\hline $\mathrm{C}$ & 4.182437 & -0.087209 & -1.532527 \\
\hline $\mathrm{H}$ & 3.393444 & -0.805701 & -1.290094 \\
\hline $\mathrm{H}$ & 4.426916 & -0.224108 & -2.591103 \\
\hline $\mathrm{H}$ & 5.065814 & -0.305476 & -0.931203 \\
\hline 0 & 1.287735 & 3.745123 & -2.262210 \\
\hline $\mathrm{C}$ & 0.351812 & 4.617404 & -1.573092 \\
\hline $\mathrm{C}$ & 1.040286 & 5.270666 & -0.382751 \\
\hline $\mathrm{H}$ & 0.032027 & 5.335717 & -2.328165 \\
\hline $\mathrm{H}$ & -0.500597 & 4.005014 & -1.269282 \\
\hline $\mathrm{C}$ & 1.452417 & 4.183579 & 0.622408 \\
\hline $\mathrm{H}$ & 1.914889 & 5.825096 & -0.744440 \\
\hline $\mathrm{H}$ & 0.600693 & 3.910845 & 1.256936 \\
\hline $\mathrm{H}$ & 2.230309 & 4.548896 & 1.307002 \\
\hline $\mathrm{C}$ & 4.521151 & 1.646169 & 1.061837 \\
\hline $\mathrm{C}$ & 4.009939 & 0.452510 & 1.609078 \\
\hline $\mathrm{C}$ & 5.458994 & 2.394340 & 1.796138 \\
\hline $\mathrm{C}$ & 4.450715 & 0.017437 & 2.854309 \\
\hline $\mathrm{H}$ & 3.239594 & -0.094931 & 1.074325 \\
\hline $\mathrm{C}$ & 5.908294 & 1.943692 & 3.033593 \\
\hline $\mathrm{H}$ & 5.840064 & 3.324532 & 1.381932 \\
\hline $\mathrm{C}$ & 5.403996 & 0.753532 & 3.564039 \\
\hline $\mathrm{H}$ & 4.041515 & -0.894162 & 3.277780 \\
\hline $\mathrm{H}$ & 6.642867 & 2.520718 & 3.587737 \\
\hline $\mathrm{H}$ & 5.744642 & 0.406304 & 4.535494 \\
\hline $\mathrm{H}$ & 0.363363 & 5.988858 & 0.088411 \\
\hline $\mathrm{H}$ & 4.291210 & 3.179483 & -0.435935 \\
\hline $\mathrm{H}$ & 1.854015 & 2.003615 & 0.451666 \\
\hline 0 & 2.209409 & 1.222665 & -3.216469 \\
\hline $\mathrm{H}$ & 1.202239 & 1.150398 & -3.109683 \\
\hline \multicolumn{4}{|c|}{1 imaginary frequency } \\
\hline \multicolumn{4}{|c|}{$E_{\text {toluene }}=-2913.025254$} \\
\hline \multicolumn{4}{|c|}{$\mathrm{G}_{\text {toluene }}=-$} \\
\hline
\end{tabular}

$\begin{array}{lrrr}\mathbf{5} & & & \\ \mathrm{C} & 1.582021 & 4.394918 & -3.469799 \\ \mathrm{C} & 0.592382 & 4.778666 & -2.367896 \\ \mathrm{C} & -0.028825 & 3.519928 & -1.759263 \\ \mathrm{C} & 1.000473 & 2.483531 & -1.343058 \\ \mathrm{C} & 2.261315 & 2.448086 & -1.964638 \\ \mathrm{C} & 2.726477 & 3.562913 & -2.885132 \\ \mathrm{C} & 0.659217 & 1.463755 & -0.428995 \\ \mathrm{C} & 1.527388 & 0.380079 & -0.270039 \\ \mathrm{C} & 2.779993 & 0.309795 & -0.886045 \\ \mathrm{C} & 3.129420 & 1.382126 & -1.710300 \\ \mathrm{C} & -0.611687 & 1.483443 & 0.351595 \\ \mathrm{C} & -0.885387 & 2.496976 & 1.299048 \\ \mathrm{C} & -2.119568 & 2.484997 & 1.970559 \\ \mathrm{C} & -3.037923 & 1.461240 & 1.713974\end{array}$




\begin{tabular}{|c|c|c|c|}
\hline & -2.759613 & 0.402234 & 0.848387 \\
\hline & -1.521248 & 0.435629 & 0.195544 \\
\hline & 0.172574 & 3.536804 & 1.629686 \\
\hline & -0.084198 & 4.246189 & 2.962141 \\
\hline & -1.532434 & 4.733434 & 3.021097 \\
\hline & -2.479198 & 3.531368 & 3.010762 \\
\hline & 1.105595 & -0.693865 & 0.525848 \\
\hline & -1.172059 & -0.634097 & -0.634468 \\
\hline & -0.021623 & -1.696680 & -0.130547 \\
\hline & 0.464721 & -2.895615 & -1.550944 \\
\hline & -0.624682 & -2.481482 & 1.731344 \\
\hline & 1.055201 & 3.812486 & -4.238030 \\
\hline & 1.985335 & 5.285511 & -3.965312 \\
\hline & 1.115750 & 5.349144 & -1.587161 \\
\hline & -0.198559 & 5.427532 & -2.761350 \\
\hline & -0.690933 & 3.775020 & -0.930730 \\
\hline & -0.678579 & 3.048005 & -2.512412 \\
\hline & 3.390798 & 4.227807 & -2.312541 \\
\hline & 3.343836 & 3.141105 & -3.687109 \\
\hline & 4.105045 & 1.365531 & -2.189119 \\
\hline & -3.996578 & 1.468885 & 2.226434 \\
\hline & 1.157498 & 3.057187 & 1.631270 \\
\hline & 0.217915 & 4.297061 & 0.838529 \\
\hline & 0.101865 & 3.555308 & 3.795907 \\
\hline & 0.618600 & 5.079502 & 3.074637 \\
\hline & -1.732926 & 5.375594 & 2.152026 \\
\hline & -1.711551 & 5.341469 & 3.915019 \\
\hline & -3.515301 & 3.856249 & 2.853640 \\
\hline & -2.460970 & 3.055947 & 4.002576 \\
\hline & -1.430820 & -3.408664 & 1.175214 \\
\hline & -3.703762 & -0.734589 & 0.700546 \\
\hline & -4.081006 & -1.429238 & 1.836025 \\
\hline & -4.944227 & -2.544545 & 1.764746 \\
\hline & -5.429287 & -2.963156 & 0.548288 \\
\hline & -5.090490 & -2.269786 & -0.642516 \\
\hline & -5.601584 & -2.676441 & -1.903533 \\
\hline & -5.288095 & -1.984899 & -3.049508 \\
\hline & -4.452109 & -0.845572 & -2.980438 \\
\hline C & -3.936046 & -0.429092 & -1.775430 \\
\hline $\boldsymbol{\gamma}$ & -4.223427 & -1.129954 & -0.574225 \\
\hline 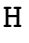 & -6.251729 & -3.546840 & -1.943137 \\
\hline 1 & -5.684311 & -2.306536 & -4.008377 \\
\hline $\mathrm{H}$ & -4.215693 & -0.296710 & -3.887415 \\
\hline $\mathrm{H}$ & -3.301420 & 0.448351 & -1.734246 \\
\hline $\mathrm{H}$ & -6.084114 & -3.828185 & 0.480147 \\
\hline $\mathrm{H}$ & -5.209705 & -3.074682 & 2.674892 \\
\hline $\mathrm{H}$ & -3.670560 & -1.128285 & 2.794819 \\
\hline C & 3.673596 & -0.865929 & -0.730721 \\
\hline C & 4.033583 & -1.578093 & -1.859122 \\
\hline C & 4.859511 & -2.720191 & -1.774471 \\
\hline $\mathrm{C}$ & 5.322901 & -3.146604 & -0.553047 \\
\hline C & 4.994072 & -2.439280 & 0.632229 \\
\hline C & 5.477038 & -2.857368 & 1.900388 \\
\hline C & 5.170002 & -2.154919 & 3.041827 \\
\hline C & 4.368874 & -0.991680 & 2.960461 \\
\hline $\mathrm{C}$ & 3.881970 & -0.562542 & 1.747352 \\
\hline C & 4.164197 & -1.273654 & 0.550998 \\
\hline $\mathrm{H}$ & 3.638799 & -1.271134 & -2.822569 \\
\hline
\end{tabular}




$\begin{array}{lrcr}\mathrm{H} & 5.110028 & -3.266460 & -2.679037 \\ \mathrm{H} & 5.948265 & -4.032380 & -0.475540 \\ \mathrm{H} & 6.099701 & -3.747224 & 1.949813 \\ \mathrm{H} & 5.544975 & -2.486320 & 4.006018 \\ \mathrm{H} & 4.138186 & -0.433328 & 3.863373 \\ \mathrm{H} & 3.276042 & 0.334996 & 1.695671 \\ 0 & \text { imaginary frequencies } & \\ \mathrm{E}_{\text {chloroform }}=-2832.207820 & \\ \mathrm{G}_{\mathrm{chloroform}}=-2831.637859 & \end{array}$

\section{TS2-up}

\begin{tabular}{|c|c|c|c|}
\hline C & -4.999512 & -4.636855 & -1.785139 \\
\hline $\mathrm{C}$ & -4.868985 & -3.480761 & -2.777979 \\
\hline C & -4.236232 & -2.263575 & -2.099523 \\
\hline C & -2.987321 & -2.595741 & -1.300639 \\
\hline C & -2.735741 & -3.910670 & -0.873962 \\
\hline C & -3.611405 & -5.072076 & -1.309672 \\
\hline $\mathrm{C}$ & -2.115748 & -1.564396 & -0.88952 \\
\hline $\mathrm{C}$ & -1.084719 & -1.852745 & 0.00644 \\
\hline C & -0.795750 & -3.159839 & 0.417754 \\
\hline C & -1.635809 & -4.170940 & -0.048848 \\
\hline C & -2.275254 & -0.153640 & -1.347262 \\
\hline $\mathrm{C}$ & -2.102289 & 0.215536 & -2.698651 \\
\hline $\mathrm{C}$ & -2.334793 & 1.548531 & -3.07410 \\
\hline C & -2.722404 & 2.478989 & -2.10452 \\
\hline $\mathrm{C}$ & -2.814121 & 2.159113 & -0.74847 \\
\hline $\mathrm{C}$ & -2.540309 & 0.831476 & -0.390134 \\
\hline C & -1.532954 & -0.785835 & -3.690401 \\
\hline C & -1.705402 & -0.367257 & -5.15236 \\
\hline $\mathrm{C}$ & -1.288022 & 1.091761 & -5.34450 \\
\hline C & -2.195988 & 2.003437 & -4.51562 \\
\hline $\mathrm{O}$ & -0.320481 & -0.796778 & 0.476050 \\
\hline $\mathrm{O}$ & -2.501102 & 0.471569 & 0.94673 \\
\hline $\mathrm{P}$ & -0.990238 & 0.301390 & 1.66161 \\
\hline S & -1.235499 & -0.610711 & 3.3683 \\
\hline S & 0.132866 & 1.916766 & 1.41064 \\
\hline $\mathrm{H}$ & -5.599613 & -4.308900 & -0.9253 \\
\hline $\mathrm{H}$ & -5.523954 & -5.487317 & -2.23640 \\
\hline $\mathrm{H}$ & -4.243784 & -3.799501 & -3.62461 \\
\hline $\mathrm{H}$ & -5.846242 & -3.205107 & $-3.1911 \varepsilon$ \\
\hline $\mathrm{H}$ & -4.030433 & -1.482682 & -2.8351 \\
\hline $\mathrm{H}$ & -4.965215 & -1.819706 & -1.4046 \\
\hline $\mathrm{H}$ & -3.107972 & -5.606283 & -2.1301 \\
\hline $\mathrm{H}$ & -3.694652 & -5.793647 & -0.487 \\
\hline $\mathrm{H}$ & -1.431573 & -5.194780 & 0.2550 \\
\hline $\mathrm{H}$ & -2.931971 & 3.502318 & -2.4063 \\
\hline $\mathrm{H}$ & -0.458778 & -0.886052 & -3.4688 \\
\hline $\mathrm{H}$ & -1.955102 & -1.780805 & -3.52971 \\
\hline $\mathrm{H}$ & -1.119790 & -1.032664 & -5.79796 \\
\hline $\mathrm{H}$ & -2.757845 & -0.482650 & -5.4486 \\
\hline $\mathrm{H}$ & -0.244923 & 1.215114 & -5.0199 \\
\hline $\mathrm{H}$ & -1.331826 & 1.378049 & -6.40210 \\
\hline $\mathrm{H}$ & -1.829206 & 3.036818 & -4.53867 \\
\hline $\mathrm{H}$ & -3.192623 & 2.027033 & -4.98249 \\
\hline $\mathrm{H}$ & 0.721728 & -0.174830 & -0.5618 \\
\hline C & -3.142692 & 3.203857 & 0.253 \\
\hline $\mathrm{C}$ & -2.354097 & 4.336953 & 0.317 \\
\hline
\end{tabular}




\begin{tabular}{|c|c|c|c|}
\hline & -2.618445 & 5.366944 & 1.245489 \\
\hline & -3.679127 & 5.258861 & 2.112628 \\
\hline & -4.527072 & 4.122172 & 2.076963 \\
\hline & -5.637176 & 4.002372 & 2.954312 \\
\hline & -6.467696 & 2.908161 & 2.900130 \\
\hline & -6.224659 & 1.884948 & 1.953961 \\
\hline & -5.155461 & 1.969032 & 1.092598 \\
\hline & -4.267319 & 3.076597 & 1.131063 \\
\hline & -5.817953 & 4.798844 & 3.672253 \\
\hline & -7.312025 & 2.827237 & 3.579180 \\
\hline & -6.886519 & 1.024520 & 1.910241 \\
\hline & -4.981400 & 1.179937 & 0.370796 \\
\hline & -3.884563 & 6.040650 & 2.839821 \\
\hline & -1.967092 & 6.235774 & 1.279763 \\
\hline & -1.489277 & 4.408296 & -0.333753 \\
\hline & 0.359295 & -3.478958 & 1.300898 \\
\hline & 0.137440 & -3.881135 & 2.602692 \\
\hline & 1.211399 & -4.199850 & 3.464923 \\
\hline & 2.510585 & -4.096966 & 3.024734 \\
\hline & 2.788535 & -3.679156 & 1.697139 \\
\hline & 4.120455 & -3.516532 & 1.231566 \\
\hline & 4.376424 & -3.097027 & -0.054881 \\
\hline & 3.302208 & -2.846292 & -0.941809 \\
\hline & 2.000520 & -2.998024 & -0.520552 \\
\hline & 1.700125 & -3.388655 & 0.810604 \\
\hline & -0.880303 & -3.913645 & 2.975071 \\
\hline & 1.000996 & -4.507507 & 4.485055 \\
\hline & 3.341993 & -4.323205 & 3.688199 \\
\hline & 4.938644 & -3.738008 & 1.913406 \\
\hline & 5.401188 & -2.984039 & -0.400247 \\
\hline 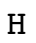 & 3.506498 & -2.533998 & -1.962076 \\
\hline & 1.183227 & -2.797881 & -1.203551 \\
\hline & 3.778570 & 1.277165 & 1.215817 \\
\hline V & 2.824961 & 0.370559 & 0.737719 \\
\hline $\boldsymbol{\gamma}$ & 2.565752 & 0.375808 & -0.687466 \\
\hline$C$ & 3.648758 & 0.826868 & -1.436789 \\
\hline$C$ & 4.893847 & 0.835382 & -0.737972 \\
\hline O & 1.434182 & 0.016342 & -1.249901 \\
\hline 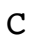 & 3.459262 & 1.223372 & -2.876054 \\
\hline$\dot{H}$ & 5.168631 & -0.063083 & -0.195674 \\
\hline $\mathrm{H}$ & 2.939839 & 0.412870 & -3.400531 \\
\hline $\mathrm{H}$ & 2.821422 & 2.110497 & -2.970623 \\
\hline $\mathrm{H}$ & 4.409854 & 1.413547 & -3.376992 \\
\hline 0 & 2.297462 & -0.589575 & 1.470761 \\
\hline V & 2.446668 & -0.469667 & 2.912080 \\
\hline 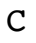 & 3.910050 & -0.209752 & 3.235018 \\
\hline $\mathrm{H}$ & 1.785998 & 0.340687 & 3.233661 \\
\hline $\mathrm{H}$ & 2.074683 & -1.414979 & 3.298518 \\
\hline C & 4.341938 & 1.120472 & 2.597496 \\
\hline $\mathrm{H}$ & 4.507615 & -1.040456 & 2.841243 \\
\hline $\mathrm{H}$ & 3.991879 & 1.965939 & 3.205835 \\
\hline $\mathrm{H}$ & 5.436606 & 1.211600 & 2.558385 \\
\hline $\mathrm{H}$ & 3.820556 & 2.259667 & 0.769866 \\
\hline C & 6.008581 & 1.725139 & -1.030105 \\
\hline C & 5.814559 & 3.008366 & -1.577834 \\
\hline C & 7.316760 & 1.320069 & -0.704638 \\
\hline C & 6.899074 & 3.844472 & -1.817104 \\
\hline $\mathrm{H}$ & 4.807729 & 3.350251 & -1.795502 \\
\hline
\end{tabular}




$\begin{array}{lrrr}\mathrm{C} & 8.401385 & 2.155966 & -0.948803 \\ \mathrm{H} & 7.472529 & 0.337489 & -0.266560 \\ \mathrm{C} & 8.194656 & 3.419077 & -1.508279 \\ \mathrm{H} & 6.736916 & 4.832590 & -2.236920 \\ \mathrm{H} & 9.406103 & 1.827140 & -0.701165 \\ \mathrm{H} & 9.039702 & 4.075114 & -1.694792 \\ \mathrm{H} & 4.053672 & -0.181149 & 4.319879 \\ 1 & \text { imaginary frequency } & \\ \mathrm{E}_{\text {chloroform }}=-3563.751846 & \\ \mathrm{G}_{\text {chloroform }}=-3562.914542 & \end{array}$

\section{TS2-down}

$\begin{array}{lrrr}\text { C } & -4.605200 & -4.228857 & 2.959415 \\ \mathrm{C} & -4.542040 & -2.746707 & 3.330521 \\ \mathrm{C} & -4.426563 & -1.897705 & 2.061608 \\ \mathrm{C} & -3.338179 & -2.385464 & 1.119300 \\ \mathrm{C} & -2.785539 & -3.669618 & 1.262209 \\ \mathrm{C} & -3.286131 & -4.650562 & 2.308597 \\ \mathrm{C} & -2.819559 & -1.523867 & 0.125830 \\ \mathrm{C} & -1.697204 & -1.920586 & -0.607302 \\ \mathrm{C} & -1.111134 & -3.186127 & -0.459954 \\ \mathrm{C} & -1.707051 & -4.052711 & 0.458218 \\ \mathrm{C} & -3.383934 & -0.160253 & -0.104388 \\ \mathrm{C} & -4.712755 & 0.040657 & -0.531575 \\ \mathrm{C} & -5.215081 & 1.351836 & -0.609916 \\ \mathrm{C} & -4.372458 & 2.429557 & -0.330805 \\ \mathrm{C} & -3.020414 & 2.267168 & -0.010446 \\ \mathrm{C} & -2.549713 & 0.950632 & 0.088654 \\ \mathrm{C} & -5.545650 & -1.125307 & -1.035362 \\ \mathrm{C} & -7.050338 & -0.849555 & -1.013680 \\ \mathrm{C} & -7.349791 & 0.482081 & -1.705201 \\ \mathrm{C} & -6.667751 & 1.629937 & -0.956003 \\ \mathrm{O} & -1.133588 & -0.999714 & -1.480777 \\ \mathrm{O} & -1.220106 & 0.728792 & 0.397588 \\ \mathrm{P} & -0.176920 & 0.184631 & -0.790773 \\ \mathrm{~S} & 1.328461 & -0.608642 & 0.207683 \\ \mathrm{~S} & 0.015103 & 1.578922 & -2.200669 \\ \mathrm{H} & -5.436692 & -4.389374 & 2.258713 \\ \mathrm{H} & -4.804329 & -4.852031 & 3.838994 \\ \mathrm{H} & -3.672358 & -2.571102 & 3.978809 \\ \mathrm{H} & -5.429902 & -2.442237 & 3.896835 \\ \mathrm{H} & -5.401061 & -1.904256 & 1.555120 \\ \mathrm{H} & -4.235889 & -0.849066 & 2.314677 \\ \mathrm{H} & -3.379768 & -5.646467 & 1.857215 \\ \mathrm{H} & -2.519828 & -4.747887 & 3.092134 \\ \mathrm{H} & -1.291337 & -5.050406 & 0.576174 \\ \mathrm{H} & -4.770982 & 3.439436 & -0.388290 \\ \mathrm{H} & -5.237693 & -1.317117 & -2.074746 \\ \mathrm{H} & -5.310145 & -2.042861 & -0.494738 \\ \mathrm{H} & -7.584582 & -1.672615 & -1.502725 \\ \mathrm{H} & -7.406106 & -0.810230 & 0.026116 \\ \mathrm{H} & -6.977525 & 0.440837 & -2.738073 \\ \mathrm{H} & -8.429593 & 0.663573 & -1.761795 \\ \mathrm{H} & -6.735831 & 2.559444 & -1.534131 \\ \mathrm{C} & -7.218388 & 1.816407 & -0.021181 \\ \mathrm{C} & -2.145457 & 3.462490 & 0.122549 \\ \mathrm{C} & -2.158271 & 4.388579 & -0.906701 \\ & -1.381668 & 5.565475 & -0.858736\end{array}$




\begin{tabular}{|c|c|c|c|}
\hline 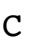 & -0.574360 & 5.816236 & 0.225511 \\
\hline & -0.517909 & 4.898032 & 1.305666 \\
\hline & 0.314444 & 5.140661 & 2.431687 \\
\hline r & 0.355282 & 4.257989 & 3.485963 \\
\hline & -0.456363 & 3.099117 & 3.465109 \\
\hline & -1.276458 & 2.838380 & 2.391639 \\
\hline & -1.320680 & 3.708479 & 1.269530 \\
\hline & 0.913921 & 6.048313 & 2.446787 \\
\hline & 0.997509 & 4.454035 & 4.340202 \\
\hline & -0.432855 & 2.410462 & 4.304524 \\
\hline & -1.895646 & 1.951109 & 2.392973 \\
\hline & 0.030838 & 6.718777 & 0.272928 \\
\hline & -1.419376 & 6.264004 & -1.689908 \\
\hline & -2.759487 & 4.182026 & -1.785833 \\
\hline & 0.089965 & -3.578430 & -1.242452 \\
\hline C & 0.055699 & -3.508810 & -2.622568 \\
\hline$C$ & 1.168678 & -3.877481 & -3.408355 \\
\hline$C$ & 2.324528 & -4.317853 & -2.808867 \\
\hline & 2.420468 & -4.383755 & -1.394859 \\
\hline & 3.626986 & -4.781415 & -0.757709 \\
\hline C & 3.734821 & -4.793620 & 0.613047 \\
\hline C & 2.632842 & -4.396716 & 1.407856 \\
\hline & 1.449490 & -4.008984 & 0.821380 \\
\hline & 1.294516 & -4.005167 & -0.591586 \\
\hline$y$ & -0.847457 & -3.156453 & -3.108939 \\
\hline 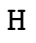 & 1.102335 & -3.811611 & -4.490490 \\
\hline H & 3.186168 & -4.606259 & -3.405963 \\
\hline $\mathrm{H}$ & 4.470665 & -5.068289 & -1.380916 \\
\hline & 4.664765 & -5.096446 & 1.086516 \\
\hline $\mathrm{H}$ & 2.723209 & -4.389308 & 2.490955 \\
\hline $\mathrm{H}$ & 0.627679 & -3.666899 & 1.437517 \\
\hline C & 3.169505 & 2.199221 & -0.064000 \\
\hline C & 3.053867 & 2.255762 & -1.457648 \\
\hline C & 3.359165 & 1.053053 & -2.178204 \\
\hline C & 4.216380 & 0.198086 & -1.502282 \\
\hline C & 4.877908 & 0.792020 & -0.377590 \\
\hline C & 4.321945 & -1.242003 & -1.911878 \\
\hline $\mathrm{H}$ & 3.394890 & -1.770654 & -1.663391 \\
\hline $\mathrm{H}$ & 4.437931 & -1.299743 & -2.998688 \\
\hline $\mathrm{H}$ & 5.160420 & -1.748947 & -1.431748 \\
\hline 0 & 2.779308 & 3.367928 & -2.142111 \\
\hline C & 2.191503 & 4.428306 & -1.343437 \\
\hline C & 3.073407 & 4.712439 & -0.133557 \\
\hline $\mathrm{H}$ & 2.117771 & 5.278521 & -2.022053 \\
\hline $\mathrm{H}$ & 1.187992 & 4.102386 & -1.055400 \\
\hline C & 3.150819 & 3.457502 & 0.753443 \\
\hline $\mathrm{H}$ & 4.069361 & 5.008919 & -0.484695 \\
\hline $\mathrm{H}$ & 2.279581 & 3.412303 & 1.419056 \\
\hline $\mathrm{H}$ & 4.028881 & 3.490837 & 1.413527 \\
\hline C & 5.299939 & 0.067709 & 0.814782 \\
\hline C & 4.679626 & -1.126379 & 1.234535 \\
\hline C & 6.334716 & 0.607304 & 1.603370 \\
\hline C & 5.101887 & -1.762285 & 2.396562 \\
\hline $\mathrm{H}$ & 3.857870 & -1.544756 & 0.667353 \\
\hline C & 6.754969 & -0.033148 & 2.763664 \\
\hline $\mathrm{H}$ & 6.809665 & 1.534475 & 1.291439 \\
\hline C & 6.138859 & -1.222498 & 3.161526 \\
\hline $\mathrm{H}$ & 4.609952 & -2.678601 & 2.704962 \\
\hline
\end{tabular}




$\begin{array}{lrrr}\mathrm{H} & 7.558320 & 0.391933 & 3.358077 \\ \mathrm{H} & 6.462516 & -1.723357 & 4.069528 \\ \mathrm{H} & 2.658713 & 5.551544 & 0.434007 \\ \mathrm{H} & 5.425686 & 1.712042 & -0.551465 \\ \mathrm{H} & 2.779022 & 1.296956 & 0.402649 \\ \mathrm{O} & 2.810619 & 0.752832 & -3.353116 \\ \mathrm{H} & 1.860684 & 1.058909 & -3.308549 \\ 1 & \text { imaginary frequency } & \\ \mathrm{E}_{\text {chloroform }}=-3563.751048 & \\ \mathrm{G}_{\text {chloroform }}=-3562.913303 & \end{array}$

\section{TS2-up, reoptimized in SMD implicit chloroform}

$\begin{array}{lrrr}\mathrm{C} & -4.796948 & -4.471229 & -2.417109 \\ \mathrm{C} & -4.597118 & -3.230997 & -3.288492 \\ \mathrm{C} & -4.029320 & -2.078357 & -2.456986 \\ \mathrm{C} & -2.848770 & -2.478706 & -1.588135 \\ \mathrm{C} & -2.618306 & -3.827906 & -1.267410 \\ \mathrm{C} & -3.448372 & -4.946177 & -1.872332 \\ \mathrm{C} & -2.022018 & -1.487765 & -1.012081 \\ \mathrm{C} & -1.066729 & -1.858115 & -0.063527 \\ \mathrm{C} & -0.784773 & -3.197880 & 0.230119 \\ \mathrm{C} & -1.575795 & -4.162520 & -0.394865 \\ \mathrm{C} & -2.154661 & -0.042045 & -1.360347 \\ \mathrm{C} & -1.871627 & 0.444098 & -2.656304 \\ \mathrm{C} & -2.098226 & 1.802358 & -2.936807 \\ \mathrm{C} & -2.600363 & 2.638731 & -1.933679 \\ \mathrm{C} & -2.808187 & 2.200899 & -0.623267 \\ \mathrm{C} & -2.521549 & 0.855011 & -0.352628 \\ \mathrm{C} & -1.207589 & -0.462266 & -3.679393 \\ \mathrm{C} & -1.252207 & 0.084292 & -5.108240 \\ \mathrm{C} & -0.842205 & 1.557193 & -5.132675 \\ \mathrm{C} & -1.835209 & 2.384780 & -4.313789 \\ \mathrm{O} & -0.352633 & -0.848436 & 0.573759 \\ \mathrm{O} & -2.580839 & 0.383454 & 0.951717 \\ \mathrm{P} & -1.127618 & 0.134368 & 1.759531 \\ \mathrm{~S} & -1.572511 & -0.906992 & 3.365964 \\ \mathrm{~S} & -0.006051 & 1.765829 & 1.793188 \\ \mathrm{H} & -5.467649 & -4.223470 & -1.582855 \\ \mathrm{H} & -5.274022 & -5.278794 & -2.984764 \\ \mathrm{H} & -3.905521 & -3.471826 & -4.108624 \\ \mathrm{H} & -5.541352 & -2.917789 & -3.749104 \\ \mathrm{H} & -3.766597 & -1.239840 & -3.105793 \\ \mathrm{H} & -4.816406 & -1.695033 & -1.790028 \\ \mathrm{H} & -2.875767 & -5.404855 & -2.692733 \\ \mathrm{H} & -3.590267 & -5.736430 & -1.125493 \\ \mathrm{H} & -1.377699 & -5.210780 & -0.185030 \\ \mathrm{H} & -2.813497 & 3.678055 & -2.171400 \\ \mathrm{H} & -0.157321 & -0.579842 & -3.371817 \\ \mathrm{H} & -1.635194 & -1.467116 & -3.647367 \\ \mathrm{H} & -0.597234 & -0.517236 & -5.749708 \\ \mathrm{H} & -2.270482 & -0.013383 & -5.510705 \\ \mathrm{H} & 0.165866 & 1.660758 & -4.706715 \\ \mathrm{H} & -0.798654 & 1.936215 & -6.160589 \\ \mathrm{H} & -1.483764 & 3.418308 & -4.209683 \\ \mathrm{H} & -2.786372 & 2.442384 & -4.864361 \\ \mathrm{H} & 0.782347 & -0.114456 & -0.397868 \\ \mathrm{C} & -3.287338 & 3.141898 & 0.421162 \\ \mathrm{C} & -2.582131 & 4.309243 & 0.650764 \\ & & & \end{array}$




\begin{tabular}{|c|c|c|c|}
\hline & -2.996790 & 5.244165 & 1.624572 \\
\hline & -4.125924 & 5.007715 & 2.372763 \\
\hline & -4.896275 & 3.834243 & 2.162262 \\
\hline & -6.078232 & 3.586146 & 2.911183 \\
\hline & -6.837175 & 2.461607 & 2.684408 \\
\hline & -6.447081 & 1.537037 & 1.686383 \\
\hline & -5.304167 & 1.745201 & 0.948082 \\
\hline & -4.485162 & 2.886093 & 1.166922 \\
\hline & -6.372494 & 4.309021 & 3.668227 \\
\hline & -7.738200 & 2.282305 & 3.264606 \\
\hline & -7.054861 & 0.655388 & 1.502534 \\
\hline & -5.023328 & 1.031685 & 0.182313 \\
\hline & -4.447457 & 5.714179 & 3.133960 \\
\hline & -2.408156 & 6.142837 & 1.786911 \\
\hline & -1.671299 & 4.491203 & 0.089257 \\
\hline & 0.338031 & -3.597749 & 1.122927 \\
\hline & 0.080782 & -4.128039 & 2.371346 \\
\hline & 1.131733 & -4.526254 & 3.230229 \\
\hline & 2.442121 & -4.388359 & 2.834493 \\
\hline & 2.755495 & -3.857060 & 1.555448 \\
\hline & 4.101029 & -3.691247 & 1.129848 \\
\hline & 4.392913 & -3.175777 & -0.113574 \\
\hline & 3.343416 & -2.816453 & -0.993028 \\
\hline & 2.029547 & -2.964026 & -0.608561 \\
\hline & 1.692395 & -3.467659 & 0.675930 \\
\hline & -0.947285 & -4.213995 & 2.706198 \\
\hline & 0.893845 & -4.930629 & 4.209980 \\
\hline & 3.255860 & -4.681374 & 3.493396 \\
\hline & 4.898908 & -3.989172 & 1.806032 \\
\hline & 5.426582 & -3.060991 & -0.429552 \\
\hline & 3.576685 & -2.425425 & -1.979397 \\
\hline & 1.234415 & -2.684513 & -1.289751 \\
\hline & 3.924057 & 1.192138 & 1.356334 \\
\hline V & 2.926352 & 0.351923 & 0.841660 \\
\hline $\boldsymbol{\gamma}$ & 2.608554 & 0.482681 & -0.554975 \\
\hline$C$ & 3.647105 & 1.018398 & -1.305142 \\
\hline$C$ & 4.920924 & 0.998740 & -0.646802 \\
\hline 0 & 1.448989 & 0.141044 & -1.095405 \\
\hline 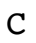 & 3.402242 & 1.529884 & -2.696821 \\
\hline $\mathrm{H}$ & 5.248709 & 0.052681 & -0.228824 \\
\hline $\mathrm{H}$ & 2.907551 & 0.745126 & -3.282597 \\
\hline $\mathrm{H}$ & 2.725641 & 2.393130 & -2.695323 \\
\hline $\mathrm{H}$ & 4.328984 & 1.805374 & -3.202276 \\
\hline 0 & 2.368596 & -0.634999 & 1.520109 \\
\hline$C$ & 2.613852 & -0.655600 & 2.953442 \\
\hline 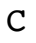 & 4.102526 & -0.488734 & 3.213477 \\
\hline $\mathrm{H}$ & 2.018938 & 0.150686 & 3.393658 \\
\hline $\mathrm{H}$ & 2.223382 & -1.617626 & 3.279025 \\
\hline C & 4.566989 & 0.872903 & 2.673964 \\
\hline $\mathrm{H}$ & 4.638063 & -1.306294 & 2.717740 \\
\hline $\mathrm{H}$ & 4.310958 & 1.673334 & 3.380872 \\
\hline $\mathrm{H}$ & 5.659472 & 0.907822 & 2.564798 \\
\hline $\mathrm{H}$ & 3.943713 & 2.223114 & 1.032650 \\
\hline C & 5.997427 & 1.948793 & -0.889297 \\
\hline C & 5.745908 & 3.274793 & -1.293757 \\
\hline C & 7.327484 & 1.547492 & -0.659845 \\
\hline C & 6.799047 & 4.161768 & -1.487187 \\
\hline $\mathrm{H}$ & 4.723114 & 3.608711 & -1.437040 \\
\hline
\end{tabular}




$\begin{array}{lrrr}\mathrm{C} & 8.379753 & 2.435782 & -0.858922 \\ \mathrm{H} & 7.524195 & 0.529471 & -0.333776 \\ \mathrm{C} & 8.116985 & 3.743589 & -1.274872 \\ \mathrm{H} & 6.595774 & 5.182758 & -1.795962 \\ \mathrm{H} & 9.402254 & 2.112915 & -0.687530 \\ \mathrm{H} & 8.936747 & 4.440037 & -1.425158 \\ \mathrm{H} & 4.298735 & -0.564068 & 4.287603 \\ \mathrm{l} & \text { imaginary frequency } & \\ \mathrm{E}_{\text {chloroform }}=-3563.753748 & \\ \mathrm{G}_{\text {chloroform }}=-3562.916684 & \end{array}$

\section{TS2-down, reoptimized in SMD implicit chloroform}

$\begin{array}{lrrr}\text { C } & -4.772741 & -4.083067 & 2.972939 \\ \mathrm{C} & -4.686784 & -2.599285 & 3.330631 \\ \mathrm{C} & -4.535844 & -1.763061 & 2.056846 \\ \mathrm{C} & -3.447076 & -2.280613 & 1.131226 \\ \mathrm{C} & -2.924055 & -3.576134 & 1.291011 \\ \mathrm{C} & -3.454449 & -4.535664 & 2.342466 \\ \mathrm{C} & -2.900951 & -1.440600 & 0.132239 \\ \mathrm{C} & -1.778670 & -1.869220 & -0.584157 \\ \mathrm{C} & -1.228988 & -3.150342 & -0.427805 \\ \mathrm{C} & -1.851428 & -3.993676 & 0.495377 \\ \mathrm{C} & -3.441014 & -0.069942 & -0.122622 \\ \mathrm{C} & -4.762439 & 0.148874 & -0.569549 \\ \mathrm{C} & -5.242277 & 1.468518 & -0.663779 \\ \mathrm{C} & -4.389989 & 2.535919 & -0.368652 \\ \mathrm{C} & -3.046821 & 2.353988 & -0.023237 \\ \mathrm{C} & -2.594543 & 1.030226 & 0.074630 \\ \mathrm{C} & -5.610299 & -1.007525 & -1.070623 \\ \mathrm{C} & -7.109392 & -0.702489 & -1.083086 \\ \mathrm{C} & -7.370232 & 0.626494 & -1.793716 \\ \mathrm{C} & -6.682319 & 1.769272 & -1.042955 \\ \mathrm{O} & -1.181202 & -0.967015 & -1.458342 \\ \mathrm{O} & -1.267135 & 0.791581 & 0.393834 \\ \mathrm{P} & -0.218567 & 0.217642 & -0.772725 \\ \mathrm{~S} & 1.280506 & -0.552951 & 0.249502 \\ \mathrm{~S} & 0.010971 & 1.570700 & -2.222648 \\ \mathrm{H} & -5.599087 & -4.236041 & 2.264454 \\ \mathrm{H} & -4.991082 & -4.693343 & 3.857035 \\ \mathrm{H} & -3.824741 & -2.433742 & 3.991704 \\ \mathrm{H} & -5.578606 & -2.271966 & 3.877735 \\ \mathrm{H} & -5.502243 & -1.753350 & 1.536235 \\ \mathrm{H} & -4.324644 & -0.717727 & 2.307716 \\ \mathrm{H} & -3.559843 & -5.534133 & 1.899750 \\ \mathrm{H} & -2.697963 & -4.636671 & 3.134659 \\ \mathrm{H} & -1.465694 & -5.001994 & 0.622866 \\ \mathrm{H} & -4.775018 & 3.550555 & -0.433476 \\ \mathrm{H} & -5.286624 & -1.223193 & -2.100462 \\ \mathrm{H} & -5.406732 & -1.919804 & -0.508674 \\ \mathrm{H} & -7.646209 & -1.521375 & -1.576382 \\ \mathrm{H} & -7.486165 & -0.646382 & -0.051676 \\ \mathrm{H} & -6.979754 & 0.567978 & -2.819069 \\ \mathrm{H} & -8.445271 & 0.827950 & -1.870666 \\ \mathrm{H} & -6.718885 & 2.693697 & -1.631724 \\ \mathrm{H} & -7.246837 & 1.976335 & -0.121099 \\ \mathrm{C} & -2.147324 & 3.526384 & 0.144669 \\ \mathrm{C} & -2.095711 & 4.462899 & -0.873963 \\ \mathrm{C} & -1.242517 & 5.585664 & -0.808378\end{array}$




\begin{tabular}{|c|c|c|c|}
\hline 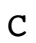 & -0.426246 & 5.770746 & 0.283128 \\
\hline & -0.453609 & 4.850891 & 1.363859 \\
\hline 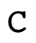 & 0.376255 & 5.033413 & 2.503808 \\
\hline 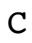 & 0.326845 & 4.156604 & 3.563233 \\
\hline & -0.573452 & 3.064677 & 3.532572 \\
\hline & -1.384206 & 2.856995 & 2.440069 \\
\hline & -1.338250 & 3.721672 & 1.313052 \\
\hline & 1.045128 & 5.890734 & 2.525150 \\
\hline $\mathrm{H}$ & 0.965635 & 4.306612 & 4.429286 \\
\hline & -0.624039 & 2.385214 & 4.378666 \\
\hline & -2.072838 & 2.020976 & 2.434841 \\
\hline & 0.245953 & 6.623708 & 0.337529 \\
\hline & -1.223452 & 6.289732 & -1.635589 \\
\hline & -2.697235 & 4.303928 & -1.763359 \\
\hline & -0.047006 & -3.588312 & -1.217036 \\
\hline & -0.096597 & -3.534307 & -2.598440 \\
\hline C & 0.993576 & -3.946810 & -3.395217 \\
\hline$C$ & 2.143728 & -4.415917 & -2.805427 \\
\hline & 2.254129 & -4.472610 & -1.391511 \\
\hline & 3.455693 & -4.905830 & -0.766756 \\
\hline C & 3.580172 & -4.912081 & 0.603143 \\
\hline C & 2.500282 & -4.477937 & 1.409676 \\
\hline C & 1.321461 & -4.057615 & 0.835040 \\
\hline I & 1.150649 & -4.050988 & -0.576619 \\
\hline & -0.996236 & -3.165094 & -3.079854 \\
\hline $\mathrm{H}$ & 0.913806 & -3.891022 & -4.477180 \\
\hline $\mathrm{f}$ & 2.989147 & -4.735791 & -3.409476 \\
\hline $\mathrm{H}$ & 4.281804 & -5.222339 & -1.398962 \\
\hline $\mathrm{H}$ & 4.506183 & -5.239166 & 1.067986 \\
\hline $\mathrm{H}$ & 2.605688 & -4.467775 & 2.491173 \\
\hline $\mathrm{H}$ & 0.515154 & -3.698949 & 1.462510 \\
\hline C & 3.415528 & 2.178803 & -0.063881 \\
\hline C & 3.218476 & 2.186408 & -1.449898 \\
\hline C & 3.420563 & 0.947786 & -2.141156 \\
\hline C & 4.270878 & 0.070495 & -1.487828 \\
\hline C & 5.019010 & 0.657903 & -0.414332 \\
\hline C & 4.296588 & -1.381678 & -1.865152 \\
\hline $\mathrm{H}$ & 3.351461 & -1.860296 & -1.586679 \\
\hline $\mathrm{H}$ & 4.389797 & -1.472465 & -2.952718 \\
\hline $\mathrm{H}$ & 5.121428 & -1.917309 & -1.393068 \\
\hline 0 & 2.961944 & 3.281981 & -2.165614 \\
\hline C & 2.570535 & 4.446361 & -1.384613 \\
\hline C & 3.569050 & 4.675296 & -0.259074 \\
\hline $\mathrm{H}$ & 2.548564 & 5.264904 & -2.104756 \\
\hline $\mathrm{H}$ & 1.559934 & 4.264938 & -1.006780 \\
\hline C & 3.560274 & 3.466685 & 0.690322 \\
\hline $\mathrm{H}$ & 4.564300 & 4.822721 & -0.695500 \\
\hline $\mathrm{H}$ & 2.721729 & 3.538483 & 1.395158 \\
\hline $\mathrm{H}$ & 4.469530 & 3.438070 & 1.305970 \\
\hline C & 5.449753 & -0.061375 & 0.777387 \\
\hline C & 4.755120 & -1.185963 & 1.267756 \\
\hline C & 6.569430 & 0.410239 & 1.489736 \\
\hline C & 5.185992 & -1.826367 & 2.424065 \\
\hline $\mathrm{H}$ & 3.871642 & -1.545409 & 0.754033 \\
\hline C & 6.999526 & -0.235847 & 2.644474 \\
\hline $\mathrm{H}$ & 7.101179 & 1.284420 & 1.122694 \\
\hline C & 6.308858 & -1.357007 & 3.11272 \\
\hline $\mathrm{H}$ & 4.639119 & -2.689112 & 2.79126 \\
\hline
\end{tabular}




$\begin{array}{lrrr}\mathrm{H} & 7.868913 & 0.133062 & 3.180668 \\ \mathrm{H} & 6.640886 & -1.860179 & 4.016407 \\ \mathrm{H} & 3.307074 & 5.590737 & 0.281332 \\ \mathrm{H} & 5.607128 & 1.541193 & -0.639697 \\ \mathrm{H} & 3.010499 & 1.326517 & 0.470693 \\ \mathrm{O} & 2.809328 & 0.648546 & -3.289857 \\ \mathrm{H} & 1.876037 & 0.997106 & -3.219343 \\ \mathrm{1} & \text { imaginary frequency } & \\ \mathrm{E}_{\text {chloroform }}=-3563.751716 & \\ \mathrm{G}_{\text {chloroform }}=-3562.915167 & \end{array}$

7

C $\quad-3.486583$

C $\quad-3.361080$

C -3.167221

C $\quad-2.101133$

C $\quad-1.695626$

C $\quad-2.192704$

C $\quad-1.577488$

C $\quad-0.754049$

C $\quad-0.303297$

C $\quad-0.783888$

C $\quad-1.890139$

C -1.459086

C $\quad-1.834093$

C $\quad-2.622820$

C $\quad-2.994135$

C $\quad-2.569790$

C $\quad-0.493193$

C $\quad-0.371552$

C $\quad-0.193020$

C $\quad-1.426294$

o -0.355630

O $\quad-2.795234$

P $\quad-1.514431$

S $\quad-1.978428$

S $\quad-0.695225$

$\mathrm{H} \quad-4.327078$

$\mathrm{H} \quad-3.706057$

$\mathrm{H} \quad-2.501876$

$\mathrm{H} \quad-4.247578$

$\mathrm{H} \quad-2.956841$

$\mathrm{H} \quad-4.114005$

$\mathrm{H} \quad-1.412193$

$\mathrm{H} \quad-2.322502$

$\mathrm{H} \quad-0.445267$

$\mathrm{H} \quad-2.939854$

$\mathrm{H} \quad 0.494134$

$\mathrm{H} \quad-0.759686$

$\mathrm{H} \quad 0.465811$

$\mathrm{H} \quad-1.279337$

$\mathrm{H} \quad 0.699863$

$\mathrm{H} \quad-0.027029$

$\mathrm{H} \quad-1.257501$

$\mathrm{H} \quad-2.263315$

$\mathrm{H} \quad 0.730057$

C $\quad-3.765486$

C $\quad-3.259503$

$$
\begin{array}{rr}
-3.968591 & -4.286254 \\
-2.507735 & -4.721605 \\
-1.603430 & -3.502302 \\
-2.102113 & -2.541533 \\
-3.447332 & -2.552636 \\
-4.421621 & -3.605819 \\
-1.236322 & -1.557052 \\
-1.755804 & -0.557314 \\
-3.080794 & -0.574553 \\
-3.902582 & -1.592748 \\
0.221193 & -1.525659 \\
1.096843 & -2.545739 \\
2.448487 & -2.482696 \\
2.894955 & -1.416951 \\
2.067009 & -0.355817 \\
0.731578 & -0.415643 \\
0.603539 & -3.610879 \\
1.544130 & -4.812216 \\
2.990338 & -4.347413 \\
3.437969 & -3.559350 \\
-0.913039 & 0.468412 \\
-0.117083 & 0.654635 \\
-0.392507 & 1.709173 \\
-1.913737 & 2.834358 \\
1.288077 & 2.341058 \\
-4.065675 & -3.585522 \\
-4.617949 & -5.141845 \\
-2.404350 & -5.400171 \\
-2.186722 & -5.280849 \\
-0.578720 & -3.817490 \\
-1.544366 & -2.944336 \\
-4.540950 & -4.372988 \\
-5.411989 & -3.153078 \\
-4.935177 & -1.629504 \\
3.934712 & -1.392307 \\
0.496364 & -3.134402 \\
-0.401781 & -3.945859 \\
1.227572 & -5.445553 \\
1.474920 & -5.428275 \\
3.057783 & -3.708917 \\
3.660248 & -5.199454 \\
4.422258 & -3.105842 \\
3.570012 & -4.261843 \\
0.014626 & 0.184220 \\
2.608404 & 0.790932 \\
3.692522 & 1.482567
\end{array}
$$




\begin{tabular}{|c|c|c|c|}
\hline $\boldsymbol{\gamma}$ & -3.949111 & 4.254184 & 2.578252 \\
\hline & -5.151993 & 3.725247 & 2.980734 \\
\hline & -5.720880 & 2.621983 & 2.294190 \\
\hline & -6.970996 & 2.074490 & 2.686511 \\
\hline & -7.527528 & 1.019092 & 2.003636 \\
\hline & -6.855165 & 0.469017 & 0.887131 \\
\hline & -5.640746 & 0.973858 & 0.484013 \\
\hline & -5.028061 & 2.052517 & 1.175582 \\
\hline & -7.481598 & 2.510697 & 3.541756 \\
\hline & -8.483264 & 0.607406 & 2.316035 \\
\hline & -7.300843 & -0.361125 & 0.346243 \\
\hline & -5.137520 & 0.545268 & -0.374508 \\
\hline & -5.685497 & 4.141884 & 3.831573 \\
\hline & -3.512661 & 5.094580 & 3.110598 \\
\hline & -2.288847 & 4.085818 & 1.199262 \\
\hline & 0.691576 & -3.579685 & 0.413921 \\
\hline & 0.300059 & -4.410336 & 1.443689 \\
\hline & 1.235298 & -4.892626 & 2.388404 \\
\hline & 2.558370 & -4.524222 & 2.308898 \\
\hline & 3.004411 & -3.662980 & 1.273029 \\
\hline 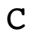 & 4.354062 & -3.227656 & 1.194560 \\
\hline & 4.769376 & -2.379861 & 0.192950 \\
\hline & 3.849754 & -1.946316 & -0.792047 \\
\hline & 2.535166 & -2.353156 & -0.744073 \\
\hline V & 2.065422 & -3.199656 & 0.295051 \\
\hline d & -0.749442 & -4.662161 & 1.545641 \\
\hline 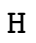 & 0.894376 & -5.540907 & 3.190269 \\
\hline $\mathrm{H}$ & 3.278194 & -4.877177 & 3.043524 \\
\hline & 5.057801 & -3.582903 & 1.943961 \\
\hline $\mathrm{H}$ & 5.807587 & -2.060563 & 0.142324 \\
\hline $\mathrm{H}$ & 4.185684 & -1.292998 & -1.592629 \\
\hline $\mathrm{H}$ & 1.834345 & -2.016214 & -1.499987 \\
\hline C & 3.389270 & 1.038067 & 2.680745 \\
\hline C & 2.687015 & 0.372016 & 1.727286 \\
\hline C & 2.498427 & 0.913542 & 0.386679 \\
\hline C & 3.464026 & 1.785127 & -0.257074 \\
\hline C & 4.783536 & 1.561952 & 0.020164 \\
\hline 0 & 1.439989 & 0.636148 & -0.280034 \\
\hline C & 2.933371 & 2.743258 & -1.295734 \\
\hline $\mathrm{H}$ & 5.006194 & 0.713825 & 0.659399 \\
\hline $\mathrm{H}$ & 1.974977 & 2.388199 & -1.678362 \\
\hline $\mathrm{H}$ & 2.760750 & 3.737003 & -0.864080 \\
\hline $\mathrm{H}$ & 3.631665 & 2.851922 & -2.129300 \\
\hline 0 & 2.015427 & -0.769870 & 1.936518 \\
\hline C & 1.771882 & -1.134924 & 3.316306 \\
\hline C & 3.043077 & -0.965419 & 4.134408 \\
\hline $\mathrm{H}$ & 0.958918 & -0.498998 & 3.678513 \\
\hline $\mathrm{H}$ & 1.427131 & -2.165273 & 3.268102 \\
\hline C & 3.502681 & 0.499148 & 4.072568 \\
\hline $\mathrm{H}$ & 3.821254 & -1.620980 & 3.726697 \\
\hline $\mathrm{H}$ & 2.893125 & 1.123230 & 4.742928 \\
\hline $\mathrm{H}$ & 4.538092 & 0.607197 & 4.423161 \\
\hline $\mathrm{H}$ & 3.823397 & 2.000267 & 2.442727 \\
\hline C & 5.960237 & 2.235756 & -0.507423 \\
\hline C & 5.966225 & 3.559684 & -0.990622 \\
\hline C & 7.175532 & 1.520579 & -0.500503 \\
\hline C & 7.140673 & 4.130670 & -1.470643 \\
\hline $\mathrm{H}$ & 5.061508 & 4.153236 & -0.957502 \\
\hline
\end{tabular}




$\begin{array}{lrrr}\mathrm{C} & 8.343800 & 2.087590 & -0.995328 \\ \mathrm{H} & 7.186410 & 0.505766 & -0.111257 \\ \mathrm{C} & 8.328488 & 3.395944 & -1.484915 \\ \mathrm{H} & 7.131191 & 5.155274 & -1.830002 \\ \mathrm{H} & 9.266880 & 1.515945 & -0.993285 \\ \mathrm{H} & 9.240994 & 3.845176 & -1.865438 \\ \mathrm{H} & 2.857504 & -1.270481 & 5.169188 \\ \text { O imaginary frequencies } & \\ \mathrm{E}_{\text {chloroform }}=-3563.768710 & \\ \mathrm{G}_{\text {chloroform }}=-3562.932533 & \end{array}$

\section{7, reoptimized in SMD implicit chloroform}

Structure 1: this structure contains an $\mathrm{OH} \cdots \mathrm{O}$ hydrogen bond, analogous to TS2-up

$\begin{array}{lrrr}\mathrm{C} & -3.413402 & -3.429512 & -4.717222 \\ \mathrm{C} & -3.259522 & -1.930481 & -4.975497 \\ \mathrm{C} & -3.091015 & -1.174944 & -3.655262 \\ \mathrm{C} & -2.062505 & -1.796760 & -2.725495 \\ \mathrm{C} & -1.669194 & -3.136677 & -2.885047 \\ \mathrm{C} & -2.146217 & -3.976371 & -4.056702 \\ \mathrm{C} & -1.559312 & -1.060439 & -1.628530 \\ \mathrm{C} & -0.777145 & -1.708605 & -0.670359 \\ \mathrm{C} & -0.324815 & -3.022321 & -0.841793 \\ \mathrm{C} & -0.782045 & -3.709803 & -1.965841 \\ \mathrm{C} & -1.850968 & 0.392164 & -1.448334 \\ \mathrm{C} & -1.382697 & 1.364772 & -2.360255 \\ \mathrm{C} & -1.758359 & 2.706123 & -2.173794 \\ \mathrm{C} & -2.586907 & 3.046268 & -1.098633 \\ \mathrm{C} & -2.999030 & 2.116354 & -0.140496 \\ \mathrm{C} & -2.566971 & 0.793538 & -0.317116 \\ \mathrm{C} & -0.392226 & 0.979080 & -3.446414 \\ \mathrm{C} & -0.244674 & 2.032917 & -4.546449 \\ \mathrm{C} & -0.070997 & 3.424799 & -3.938034 \\ \mathrm{C} & -1.316105 & 3.798752 & -3.130931 \\ \mathrm{O} & -0.412280 & -1.002980 & 0.472385 \\ \mathrm{O} & -2.840989 & -0.162400 & 0.651070 \\ \mathrm{P} & -1.594657 & -0.627475 & 1.676672 \\ \mathrm{~S} & -2.212532 & -2.258405 & 2.581612 \\ \mathrm{~S} & -0.790784 & 0.914161 & 2.618808 \\ \mathrm{H} & -4.276658 & -3.596382 & -4.058395 \\ \mathrm{H} & -3.610369 & -3.971751 & -5.649499 \\ \mathrm{H} & -2.380983 & -1.760673 & -5.614492 \\ \mathrm{H} & -4.127605 & -1.533467 & -5.514595 \\ \mathrm{H} & -2.856178 & -0.125951 & -3.848586 \\ \mathrm{H} & -4.054090 & -1.164238 & -3.122742 \\ \mathrm{H} & -1.343424 & -4.018065 & -4.808446 \\ \mathrm{H} & -2.300021 & -5.009423 & -3.722844 \\ \mathrm{H} & -0.443267 & -4.730947 & -2.122793 \\ \mathrm{H} & -2.907985 & 4.079011 & -0.987333 \\ \mathrm{H} & 0.583600 & 0.826697 & -2.960216 \\ \mathrm{H} & -0.650939 & 0.012892 & -3.885115 \\ \mathrm{H} & 0.606751 & 1.775017 & -5.187426 \\ \mathrm{H} & -1.138828 & 2.027289 & -5.185764 \\ \mathrm{H} & 0.811383 & 3.426293 & -3.282559 \\ \mathrm{H} & 0.106843 & 4.175057 & -4.717524 \\ \mathrm{H} & -1.148662 & 4.726478 & -2.570673 \\ \mathrm{H} & -2.139081 & 4.012094 & -3.829602 \\ \mathrm{H} & 0.829547 & 0.033796 & 0.353254 \\ & & & \end{array}$




\begin{tabular}{|c|c|c|c|}
\hline $\boldsymbol{\gamma}$ & -3.833536 & 2.543502 & 1.011635 \\
\hline & -3.388574 & 3.577863 & 1.815044 \\
\hline & -4.142467 & 4.033820 & 2.918581 \\
\hline & -5.350515 & 3.449674 & 3.218616 \\
\hline & -5.861500 & 2.398201 & 2.413720 \\
\hline & -7.119218 & 1.800155 & 2.696680 \\
\hline & -7.623919 & 0.801010 & 1.897419 \\
\hline & -6.890686 & 0.362511 & 0.769126 \\
\hline & -5.666659 & 0.917079 & 0.471436 \\
\hline & -5.104389 & 1.937973 & 1.285106 \\
\hline & -7.677663 & 2.152897 & 3.560382 \\
\hline & -8.586067 & 0.350399 & 2.125136 \\
\hline & -7.297667 & -0.419081 & 0.133520 \\
\hline & -5.121602 & 0.575481 & -0.400631 \\
\hline & -5.933039 & 3.783625 & 4.073594 \\
\hline & -3.752567 & 4.838401 & 3.535879 \\
\hline & -2.420960 & 4.023963 & 1.608108 \\
\hline & 0.666515 & -3.643650 & 0.079761 \\
\hline & 0.278756 & -4.608824 & 0.987178 \\
\hline & 1.216990 & -5.218045 & 1.852985 \\
\hline & 2.542613 & -4.851673 & 1.809711 \\
\hline & 2.987795 & -3.866560 & 0.889389 \\
\hline & 4.347237 & -3.455129 & 0.840638 \\
\hline & 4.765592 & -2.494089 & -0.051788 \\
\hline & 3.838230 & -1.910571 & -0.948669 \\
\hline 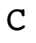 & 2.514219 & -2.288435 & -0.925911 \\
\hline 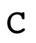 & 2.043475 & -3.259895 & -0.001680 \\
\hline $\mathrm{H}$ & -0.768565 & -4.883696 & 1.050023 \\
\hline & 0.878102 & -5.968943 & 2.560989 \\
\hline $\mathrm{H}$ & 3.266885 & -5.306241 & 2.481167 \\
\hline H & 5.054531 & -3.921128 & 1.522629 \\
\hline $\mathrm{H}$ & 5.809364 & -2.192570 & -0.081263 \\
\hline $\mathrm{H}$ & 4.175956 & -1.163778 & -1.661749 \\
\hline $\mathrm{H}$ & 1.811672 & -1.835798 & -1.616955 \\
\hline C & 3.580028 & 0.698945 & 2.789126 \\
\hline C & 2.809316 & 0.195911 & 1.789662 \\
\hline C & 2.567827 & 0.906209 & 0.545531 \\
\hline C & 3.482886 & 1.853134 & -0.038509 \\
\hline C & 4.820988 & 1.630299 & 0.168985 \\
\hline 0 & 1.457971 & 0.695555 & -0.088937 \\
\hline C & 2.901268 & 2.911240 & -0.943699 \\
\hline $\mathrm{H}$ & 5.082067 & 0.720421 & 0.698967 \\
\hline $\mathrm{H}$ & 1.894255 & 2.634399 & -1.258088 \\
\hline $\mathrm{H}$ & 2.826202 & 3.872924 & -0.421762 \\
\hline $\mathrm{H}$ & 3.519509 & 3.056324 & -1.833848 \\
\hline 0 & 2.093797 & -0.943844 & 1.879928 \\
\hline C & 1.917821 & -1.487092 & 3.208505 \\
\hline C & 3.235212 & -1.479540 & 3.967401 \\
\hline $\mathrm{H}$ & 1.155398 & -0.882661 & 3.709748 \\
\hline $\mathrm{H}$ & 1.530383 & -2.491373 & 3.044887 \\
\hline C & 3.762667 & -0.042307 & 4.074070 \\
\hline $\mathrm{H}$ & 3.958206 & -2.106089 & 3.433407 \\
\hline $\mathrm{H}$ & 3.242963 & 0.506874 & 4.873279 \\
\hline $\mathrm{H}$ & 4.825915 & -0.027618 & 4.348924 \\
\hline $\mathrm{H}$ & 4.029970 & 1.676147 & 2.670653 \\
\hline C & 5.969877 & 2.377321 & -0.307325 \\
\hline C & 5.937715 & 3.727797 & -0.717197 \\
\hline C & 7.208888 & 1.699139 & -0.328851 \\
\hline
\end{tabular}




$\begin{array}{cccr}\mathrm{C} & 7.098864 & 4.359314 & -1.150289 \\ \mathrm{H} & 5.016681 & 4.293083 & -0.667635 \\ \mathrm{C} & 8.363204 & 2.329231 & -0.776866 \\ \mathrm{H} & 7.248177 & 0.665177 & 0.002967 \\ \mathrm{C} & 8.310122 & 3.662959 & -1.191387 \\ \mathrm{H} & 7.060982 & 5.401288 & -1.453459 \\ \mathrm{H} & 9.303943 & 1.787452 & -0.796618 \\ \mathrm{H} & 9.211447 & 4.161724 & -1.535585 \\ \mathrm{H} & 3.085210 & -1.913917 & 4.960736 \\ \text { O } & \text { imaginary frequencies } & \end{array}$

\section{7 , reoptimized in SMD implicit chloroform}

Structure 2: this structure contains an $\mathrm{OH} \cdots \mathrm{S}$ hydrogen bond

$\begin{array}{lrrr}\text { C } & -5.488379 & -0.430280 & 4.612871 \\ \mathrm{C} & -6.035444 & 0.523785 & 3.550953 \\ \mathrm{C} & -4.941603 & 0.885735 & 2.544064 \\ \mathrm{C} & -4.183300 & -0.317630 & 2.007576 \\ \mathrm{C} & -4.162913 & -1.523813 & 2.731289 \\ \mathrm{C} & -5.035989 & -1.735890 & 3.955860 \\ \mathrm{C} & -3.396892 & -0.205650 & 0.837565 \\ \mathrm{C} & -2.523829 & -1.247357 & 0.500517 \\ \mathrm{C} & -2.481438 & -2.456197 & 1.210504 \\ \mathrm{C} & -3.336704 & -2.570247 & 2.309827 \\ \mathrm{C} & -3.425618 & 1.019448 & -0.020679 \\ \mathrm{C} & -4.598873 & 1.447858 & -0.683051 \\ \mathrm{C} & -4.573902 & 2.658248 & -1.399648 \\ \mathrm{C} & -3.385707 & 3.391719 & -1.480607 \\ \mathrm{C} & -2.187985 & 2.947135 & -0.914112 \\ \mathrm{C} & -2.239133 & 1.740031 & -0.201646 \\ \mathrm{C} & -5.830393 & 0.557659 & -0.721297 \\ \mathrm{C} & -7.112015 & 1.300001 & -1.105745 \\ \mathrm{C} & -6.880985 & 2.149523 & -2.355713 \\ \mathrm{C} & -5.816911 & 3.212390 & -2.074144 \\ \mathrm{O} & -1.667441 & -1.068837 & -0.575917 \\ \mathrm{O} & -1.071362 & 1.230460 & 0.344985 \\ \mathrm{P} & -0.337347 & -0.076872 & -0.387877 \\ \mathrm{~S} & 0.976039 & -0.721457 & 0.926201 \\ \mathrm{~S} & 0.172903 & 0.296285 & -2.285702 \\ \mathrm{H} & -4.637342 & 0.044017 & 5.120994 \\ \mathrm{H} & -6.243533 & -0.641683 & 5.379180 \\ \mathrm{H} & -6.875707 & 0.043790 & 3.029045 \\ \mathrm{H} & -6.426073 & 1.439668 & 4.009885 \\ \mathrm{H} & -5.359766 & 1.481923 & 1.731370 \\ \mathrm{H} & -4.206818 & 1.541194 & 3.036281 \\ \mathrm{H} & -5.927227 & -2.307496 & 3.655388 \\ \mathrm{H} & -4.502944 & -2.366573 & 4.677377 \\ \mathrm{H} & -3.336834 & -3.500438 & 2.872528 \\ \mathrm{H} & -3.383010 & 4.330353 & -2.029163 \\ \mathrm{H} & -5.639757 & -0.232027 & -1.464166 \\ \mathrm{H} & -5.967044 & 0.034131 & 0.226692 \\ \mathrm{H} & -7.919003 & 0.575554 & -1.267147 \\ \mathrm{H} & -7.426659 & 1.950302 & -0.276901 \\ \mathrm{H} & -6.548776 & 1.500236 & -3.177569 \\ \mathrm{H} & -7.810329 & 2.631277 & -2.681947 \\ \mathrm{H} & -5.530080 & 3.725618 & -2.999745 \\ \mathrm{H} & -6.252934 & 3.985100 & -1.422871 \\ \mathrm{H} & 2.127103 & 0.514552 & -1.866697\end{array}$




\begin{tabular}{|c|c|c|c|}
\hline C & -0.914162 & 3.676069 & -1.150747 \\
\hline c & -0.560102 & 3.967316 & -2.457017 \\
\hline C & 0.662471 & 4.598821 & -2.771129 \\
\hline C & 1.544294 & 4.933190 & -1.770497 \\
\hline c & 1.219002 & 4.680529 & -0.412462 \\
\hline C & 2.110562 & 5.039914 & 0.635095 \\
\hline C & 1.779258 & 4.822533 & 1.952379 \\
\hline C & 0.527365 & 4.247862 & 2.278462 \\
\hline C & -0.354077 & 3.882405 & 1.286708 \\
\hline C & -0.034534 & 4.063210 & -0.086199 \\
\hline $\mathrm{H}$ & 3.060327 & 5.499113 & 0.371252 \\
\hline $\mathrm{H}$ & 2.468809 & 5.101561 & 2.744534 \\
\hline $\mathrm{H}$ & 0.260683 & 4.094432 & 3.320453 \\
\hline $\mathrm{H}$ & -1.311435 & 3.450320 & 1.552362 \\
\hline $\mathrm{H}$ & 2.498994 & 5.399143 & -2.001917 \\
\hline $\mathrm{H}$ & 0.910106 & 4.794327 & -3.810746 \\
\hline $\mathrm{H}$ & -1.220090 & 3.657433 & -3.261034 \\
\hline C & -1.519489 & -3.542405 & 0.884759 \\
\hline C & -0.682384 & -3.999131 & 1.888277 \\
\hline c & 0.281901 & -5.002839 & 1.650912 \\
\hline C & 0.416688 & -5.548607 & 0.395309 \\
\hline C & -0.428791 & -5.128512 & -0.664992 \\
\hline C & -0.309081 & -5.689221 & -1.965875 \\
\hline C & -1.144335 & -5.296104 & -2.985735 \\
\hline C & -2.153165 & -4.334360 & -2.739292 \\
\hline C & -2.292474 & -3.7 & -1.491701 \\
\hline C & -1.427056 & -4.126273 & -0.422032 \\
\hline $\mathrm{H}$ & -0.744211 & -3.543061 & 2.871188 \\
\hline $\mathrm{H}$ & 0.925397 & -5.327612 & 2.463920 \\
\hline $\mathrm{H}$ & 1.163234 & -6.313981 & 0.197964 \\
\hline $\mathrm{H}$ & 0.454495 & -6.444516 & -2.135962 \\
\hline $\mathrm{H}$ & -1.041408 & -5.729767 & -3.976627 \\
\hline $\mathrm{H}$ & -2.822642 & -4.039395 & -3.542326 \\
\hline $\mathrm{H}$ & -3.074077 & -3.040994 & -1.317261 \\
\hline C & 4.054825 & -2.184631 & -0.171199 \\
\hline C & 3.666229 & -1.286519 & -1.112155 \\
\hline C & 3.844393 & 0.146813 & -0.959659 \\
\hline C & 4.89428 & 0.75 & -0.181238 \\
\hline C & 6.077069 & 0.080490 & -0.050250 \\
\hline 0 & 3.004579 & 0.946199 & -1.534367 \\
\hline C & 4.620365 & 2.148783 & 0.341902 \\
\hline $\mathrm{H}$ & 6.195875 & -0.818696 & -0.646874 \\
\hline $\mathrm{H}$ & 3.959832 & 2.683715 & -0.342243 \\
\hline $\mathrm{H}$ & 4.106976 & 2.110913 & 1.310264 \\
\hline $\mathrm{H}$ & 5.543864 & 2.719086 & 0.459527 \\
\hline 0 & 2.934969 & -1.609504 & -2.209144 \\
\hline C & 2.211050 & -2.861755 & -2.113685 \\
\hline C & 3.146803 & -3.975694 & -1.669657 \\
\hline $\mathrm{H}$ & 1.389388 & -2.721312 & -1.405869 \\
\hline $\mathrm{H}$ & 1.801443 & -3.026383 & -3.110425 \\
\hline C & 3.726417 & -3.636440 & -0.290541 \\
\hline $\mathrm{H}$ & 3.951445 & -4.083899 & -2.406537 \\
\hline $\mathrm{H}$ & 3.001109 & -3.882324 & 0.499261 \\
\hline $\mathrm{H}$ & 4.621153 & -4.235125 & -0.072478 \\
\hline $\mathrm{H}$ & 4.521193 & -1.827071 & 0.737434 \\
\hline C & 7.250220 & 0.433588 & 0.731272 \\
\hline C & 7.211019 & 1.241194 & 1.887569 \\
\hline C & 8.490488 & -0.108029 & 0.331465 \\
\hline
\end{tabular}




$\begin{array}{rrrr}\mathrm{C} & 8.377564 & 1.516406 & 2.594024 \\ \mathrm{H} & 6.265427 & 1.620987 & 2.253593 \\ \mathrm{C} & 9.656286 & 0.183493 & 1.030079 \\ \mathrm{H} & 8.526980 & -0.751638 & -0.543324 \\ \mathrm{C} & 9.602831 & 1.000390 & 2.162933 \\ \mathrm{H} & 8.330946 & 2.130692 & 3.488321 \\ \mathrm{H} & 10.604112 & -0.230714 & 0.699571 \\ \mathrm{H} & 10.510890 & 1.223652 & 2.715584 \\ \mathrm{H} & 2.596791 & -4.920203 & -1.635238 \\ \mathrm{O} & \text { imaginary frequencies }\end{array}$

\section{9-up}

$\begin{array}{lrrr}\text { C } & -6.789179 & -2.508115 & -0.737395 \\ \mathrm{C} & -6.396066 & -1.484712 & -1.803924 \\ \mathrm{C} & -5.206866 & -0.649252 & -1.324659 \\ \mathrm{C} & -4.068701 & -1.485429 & -0.765859 \\ \mathrm{C} & -4.292662 & -2.797226 & -0.311813 \\ \mathrm{C} & -5.634247 & -3.483676 & -0.498560 \\ \mathrm{C} & -2.785270 & -0.919067 & -0.604904 \\ \mathrm{C} & -1.817081 & -1.641430 & 0.088621 \\ \mathrm{C} & -1.995731 & -2.955943 & 0.527058 \\ \mathrm{C} & -3.254222 & -3.513466 & 0.296562 \\ \mathrm{C} & -2.414941 & 0.425416 & -1.140445 \\ \mathrm{C} & -2.392167 & 0.682326 & -2.529940 \\ \mathrm{C} & -2.080159 & 1.976698 & -2.979475 \\ \mathrm{C} & -1.769067 & 2.974203 & -2.051428 \\ \mathrm{C} & -1.690007 & 2.726277 & -0.679545 \\ \mathrm{C} & -1.994535 & 1.426341 & -0.258830 \\ \mathrm{C} & -2.555241 & -0.460517 & -3.518636 \\ \mathrm{C} & -2.861930 & 0.000777 & -4.945110 \\ \mathrm{C} & -1.899068 & 1.112132 & -5.365117 \\ \mathrm{C} & -2.084146 & 2.328886 & -4.455872 \\ \mathrm{O} & -0.600092 & -0.997937 & 0.349521 \\ \mathrm{O} & -1.843828 & 1.108198 & 1.095459 \\ \mathrm{P} & -0.589349 & 0.148789 & 1.561819 \\ \mathrm{~S} & -0.853459 & -0.491181 & 3.356369 \\ \mathrm{~S} & 1.139798 & 1.226255 & 1.044639 \\ \mathrm{H} & -7.029671 & -1.982176 & 0.196700 \\ \mathrm{H} & -7.688107 & -3.060725 & -1.033720 \\ \mathrm{H} & -6.129440 & -2.010374 & -2.732000 \\ \mathrm{H} & -7.237032 & -0.823892 & -2.043315 \\ \mathrm{H} & -4.851795 & 0.004365 & -2.124784 \\ \mathrm{H} & -5.543689 & 0.028239 & -0.525326 \\ \mathrm{H} & -5.560607 & -4.164553 & -1.360030 \\ \mathrm{H} & -5.841063 & -4.120099 & 0.370073 \\ \mathrm{H} & -3.432124 & -4.536822 & 0.616897 \\ \mathrm{H} & -1.544558 & 3.976783 & -2.405975 \\ \mathrm{H} & -1.607094 & -1.020207 & -3.519530 \\ \mathrm{H} & -3.311714 & -1.171239 & -3.179689 \\ \mathrm{H} & -2.796980 & -0.854252 & -5.627788 \\ \mathrm{H} & -3.894514 & 0.374423 & -4.999773 \\ \mathrm{H} & -0.866526 & 0.745702 & -5.286527 \\ \mathrm{H} & -2.060545 & 1.398834 & -6.410655 \\ \mathrm{H} & -1.309023 & 3.078963 & -4.652228 \\ \mathrm{H} & -3.042119 & 2.812649 & -4.700176 \\ \mathrm{H} & 0.386927 & -0.654435 & -1.060025 \\ \mathrm{C} & -1.227858 & 3.780958 & 0.257750 \\ \mathrm{C} & -0.002173 & 4.378732 & 0.024798 \\ & & & \end{array}$




\begin{tabular}{|c|c|c|c|}
\hline V & 0.508220 & 5.373632 & 0.886537 \\
\hline & -0.215254 & 5.768749 & 1.986293 \\
\hline & -1.486307 & 5.199896 & 2.258133 \\
\hline & -2.253725 & 5.612010 & 3.379607 \\
\hline & -3.495185 & 5.074170 & 3.623419 \\
\hline & -4.028085 & 4.102236 & 2.744393 \\
\hline & -3.307860 & 3.677764 & 1.651987 \\
\hline & -2.014319 & 4.196824 & 1.380423 \\
\hline & -1.838465 & 6.367304 & 4.042193 \\
\hline & -4.071676 & 5.396523 & 4.485823 \\
\hline & -5.013859 & 3.687747 & 2.935070 \\
\hline & -3.729797 & 2.937269 & 0.982635 \\
\hline & 0.173923 & 6.524204 & 2.664170 \\
\hline & 1.481190 & 5.810761 & 0.681760 \\
\hline & 0.591838 & 4.042811 & -0.819373 \\
\hline & -0.914618 & -3.698349 & 1.224369 \\
\hline & -1.126931 & -4.139510 & 2.516214 \\
\hline & -0.110775 & -4.800758 & 3.240728 \\
\hline & 1.123187 & -5.004670 & 2.669329 \\
\hline & 1.386531 & -4.575478 & 1.342151 \\
\hline C & 2.662295 & -4.754704 & 0.744624 \\
\hline C & 2.905217 & -4.336733 & -0.542902 \\
\hline C & 1.868504 & -3.745497 & -1.300340 \\
\hline & 0.622324 & -3.560155 & -0.749581 \\
\hline C & 0.348402 & -3.933990 & 0.591392 \\
\hline $\mathrm{H}$ & -2.079013 & -3.931188 & 2.993897 \\
\hline 4 & -0.305449 & -5.125353 & 4.258666 \\
\hline $\mathrm{H}$ & 1.917929 & -5.494253 & 3.227133 \\
\hline & 3.446200 & -5.230249 & 1.329456 \\
\hline $\mathrm{H}$ & 3.888425 & -4.470295 & -0.985699 \\
\hline $\mathrm{H}$ & 2.059051 & -3.422805 & -2.318828 \\
\hline $\mathrm{H}$ & -0.163396 & -3.108468 & -1.343997 \\
\hline$C$ & 3.788441 & 0.412568 & 0.616339 \\
\hline C & 2.362133 & -0.157080 & 0.447271 \\
\hline C & 2.269366 & -0.382456 & -1.048204 \\
\hline C & 3.488783 & -0.453757 & -1.605429 \\
\hline C & 4.570175 & -0.224665 & -0.570980 \\
\hline 0 & 1.105776 & -0.545658 & -1.713403 \\
\hline C & 3.781874 & -0.823817 & -3.023304 \\
\hline $\mathrm{H}$ & 4.983055 & -1.193773 & -0.250524 \\
\hline $\mathrm{H}$ & 2.855592 & -0.968501 & -3.585633 \\
\hline $\mathrm{H}$ & 4.381316 & -0.056058 & -3.527814 \\
\hline $\mathrm{H}$ & 4.363175 & -1.756064 & -3.070284 \\
\hline 0 & 2.120361 & -1.353720 & 1.110434 \\
\hline C & 2.586652 & -1.437469 & 2.468896 \\
\hline C & 4.088245 & -1.213905 & 2.529313 \\
\hline $\mathrm{H}$ & 2.054416 & -0.700904 & 3.082280 \\
\hline $\mathrm{H}$ & 2.288610 & -2.434097 & 2.793500 \\
\hline C & 4.400818 & 0.188900 & 1.999635 \\
\hline $\mathrm{H}$ & 4.587959 & -1.984823 & 1.929381 \\
\hline $\mathrm{H}$ & 3.987814 & 0.928032 & 2.697118 \\
\hline $\mathrm{H}$ & 5.480729 & 0.370047 & 1.944640 \\
\hline $\mathrm{H}$ & 3.740905 & 1.487484 & 0.411796 \\
\hline C & 5.722345 & 0.648072 & -1.017318 \\
\hline C & 5.480586 & 1.852673 & -1.691857 \\
\hline C & 7.044798 & 0.291105 & -0.739340 \\
\hline C & 6.534241 & 2.678505 & -2.076302 \\
\hline $\mathrm{H}$ & 4.454365 & 2.132891 & -1.918076 \\
\hline
\end{tabular}




$\begin{array}{lrrr}\mathrm{C} & 8.104720 & 1.117035 & -1.119059 \\ \mathrm{H} & 7.244386 & -0.644611 & -0.222018 \\ \mathrm{C} & 7.852125 & 2.313355 & -1.789262 \\ \mathrm{H} & 6.329202 & 3.607260 & -2.601938 \\ \mathrm{H} & 9.126293 & 0.823342 & -0.893453 \\ \mathrm{H} & 8.674742 & 2.956448 & -2.089126 \\ \mathrm{H} & 4.437659 & -1.322440 & 3.562914 \\ 0 & \text { imaginary frequencies } & \\ \mathrm{E}_{\text {chloroform }}=-3563.804265 & \\ \mathrm{G}_{\text {chloroform }}=-3562.962720 & \end{array}$

\section{TS3}

\begin{tabular}{|c|c|c|c|}
\hline $\mathrm{C}$ & 6.148168 & -2.422414 & -2.785531 \\
\hline C & 6.517229 & -1.528058 & -1.600998 \\
\hline C & 5.309500 & -0.692986 & -1.170533 \\
\hline C & 4.041007 & -1.510728 & -0.988730 \\
\hline $\mathrm{C}$ & 3.892782 & -2.747188 & -1.641709 \\
\hline C & 5.048853 & -3.404742 & -2.374913 \\
\hline C & 2.949813 & -0.982966 & -0.262432 \\
\hline $\mathrm{C}$ & 1.720013 & -1.645761 & -0.316039 \\
\hline C & 1.540762 & -2.868740 & -0.972503 \\
\hline C & 2.661169 & -3.409465 & -1.605102 \\
\hline C & 3.047559 & 0.284789 & 0.522898 \\
\hline C & 3.928565 & 0.419542 & 1.618792 \\
\hline C & 4.012197 & 1.658931 & 2.278163 \\
\hline C & 3.179115 & 2.709640 & 1.885670 \\
\hline C & 2.226740 & 2.578171 & 0.870914 \\
\hline C & 2.191340 & 1.345330 & 0.203723 \\
\hline C & 4.677484 & -0.788029 & 2.158985 \\
\hline C & 5.875960 & -0.420975 & 3.036684 \\
\hline C & 5.467806 & 0.610707 & 4.089223 \\
\hline C & 5.002679 & 1.897829 & 3.404308 \\
\hline 0 & 0.614271 & -1.038412 & 0.277749 \\
\hline 0 & 1.263581 & 1.159266 & -0.814998 \\
\hline$P$ & -0.069052 & 0.217557 & -0.561391 \\
\hline$S$ & -0.797500 & -0.195039 & -2.316351 \\
\hline S & -1.201099 & 1.063914 & 0.912123 \\
\hline $\mathrm{H}$ & 5.793779 & -1.794606 & -3.614 \\
\hline $\mathrm{H}$ & 7.022609 & -2.972658 & -3.151959 \\
\hline $\mathrm{H}$ & 6.852757 & -2.154594 & -0.761995 \\
\hline $\mathrm{H}$ & 7.351578 & -0.864135 & -1.855430 \\
\hline $\mathrm{H}$ & 5.542861 & -0.121621 & -0.270157 \\
\hline $\mathrm{H}$ & 5.103709 & 0.061424 & -1.945291 \\
\hline $\mathrm{H}$ & 5.487480 & -4.171747 & -1.718511 \\
\hline $\mathrm{H}$ & 4.666531 & -3.942603 & -3.250690 \\
\hline $\mathrm{H}$ & 2.557809 & -4.365229 & -2.112688 \\
\hline $\mathrm{H}$ & 3.247568 & 3.660645 & 2.407641 \\
\hline $\mathrm{H}$ & 3.963203 & -1.373540 & 2.758253 \\
\hline $\mathrm{H}$ & 4.986995 & -1.454621 & 1.352337 \\
\hline $\mathrm{H}$ & 6.276526 & -1.325692 & 3.508582 \\
\hline $\mathrm{H}$ & 6.679278 & -0.005342 & 2.411484 \\
\hline $\mathrm{H}$ & 4.652142 & 0.199792 & 4.700024 \\
\hline $\mathrm{H}$ & 6.298511 & 0.829365 & 4.770252 \\
\hline $\mathrm{H}$ & 4.564518 & 2.587065 & 4.136031 \\
\hline $\mathrm{H}$ & 5.881916 & 2.415697 & 2.991713 \\
\hline $\mathrm{H}$ & -3.047749 & -1.375101 & -3.040444 \\
\hline C & 1.226345 & 3.650828 & 0.628583 \\
\hline $\mathrm{C}$ & 0.515980 & 4.122764 & 1.719039 \\
\hline
\end{tabular}




\begin{tabular}{|c|c|c|c|}
\hline & -0.510840 & 5.079600 & 1.577732 \\
\hline & -0.837017 & 5.560145 & 0.332216 \\
\hline & -0.128187 & 5.123583 & -0.816314 \\
\hline & -0.455774 & 5.609540 & -2.110491 \\
\hline & 0.246262 & 5.198871 & -3.219199 \\
\hline & 1.326351 & 4.296241 & -3.074790 \\
\hline & 1.664212 & 3.803455 & -1.836003 \\
\hline & 0.936851 & 4.173717 & -0.673819 \\
\hline & -1.271592 & 6.322555 & -2.204632 \\
\hline & -0.014670 & 5.576377 & -4.203995 \\
\hline & 1.890870 & 3.988353 & -3.949956 \\
\hline & 2.495097 & 3.115810 & -1.738933 \\
\hline & -1.640495 & 6.282094 & 0.207587 \\
\hline & -1.055041 & 5.413040 & 2.456640 \\
\hline & 0.720090 & 3.702314 & 2.698583 \\
\hline & 0.204546 & -3.510154 & -1.075824 \\
\hline & -0.322174 & -3.735222 & -2.334315 \\
\hline & -1.612080 & -4.281446 & -2.506728 \\
\hline & -2.373643 & -4.611609 & -1.411257 \\
\hline & -1.868337 & -4.425319 & -0.099254 \\
\hline & -2.630125 & -4.787267 & 1.044237 \\
\hline & -2.128349 & -4.618131 & 2.313071 \\
\hline & -0.828459 & -4.087351 & 2.491506 \\
\hline & -0.066196 & -3.723905 & 1.405263 \\
\hline & -0.558498 & -3.870396 & 0.080781 \\
\hline & 0.253923 & -3.434659 & -3.203445 \\
\hline & -2.005014 & -4.422513 & -3.509338 \\
\hline & -3.371727 & -5.022842 & -1.534512 \\
\hline & -3.620601 & -5.208567 & 0.894160 \\
\hline & -2.723515 & -4.893907 & 3.178662 \\
\hline & -0.428181 & -3.970618 & 3.494863 \\
\hline & 0.930223 & -3.324438 & 1.553604 \\
\hline & -4.024789 & 1.122006 & -0.296670 \\
\hline & -3.879409 & 0.629908 & -1.584600 \\
\hline & -4.153923 & -0.803063 & -1.622667 \\
\hline & -4.714538 & -1.175248 & -0.446841 \\
\hline & -4.795217 & 0.031833 & 0.474395 \\
\hline & -3.940867 & -1.587148 & -2.709499 \\
\hline $\boldsymbol{\gamma}$ & -5.206939 & -2.535373 & -0.089707 \\
\hline 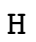 & -5.844323 & 0.368116 & 0.505659 \\
\hline 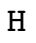 & -5.004457 & -3.235482 & -0.903338 \\
\hline 1 & -4.727011 & -2.907343 & 0.821560 \\
\hline $\mathrm{H}$ & -6.289016 & -2.524292 & 0.100017 \\
\hline 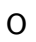 & -3.584287 & 1.309667 & -2.681988 \\
\hline I & -3.570857 & 2.761793 & -2.563353 \\
\hline & -3.227354 & 3.268495 & -1.167028 \\
\hline $\mathrm{H}$ & -2.832032 & 3.091996 & -3.295270 \\
\hline $\mathrm{H}$ & -4.564627 & 3.102021 & -2.878299 \\
\hline 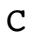 & -4.104967 & 2.613555 & -0.084844 \\
\hline t & -3.341394 & 4.357738 & -1.164744 \\
\hline $\mathrm{H}$ & -3.733484 & 2.889146 & 0.910097 \\
\hline $\mathrm{H}$ & -5.142003 & 2.974595 & -0.150120 \\
\hline $\mathrm{H}$ & -2.570954 & 0.946897 & 0.179593 \\
\hline C & -4.358187 & -0.207904 & 1.906265 \\
\hline C & -3.309831 & -1.081585 & 2.221185 \\
\hline $\mathrm{C}$ & -4.987008 & 0.489879 & 2.943390 \\
\hline C & -2.903420 & -1.249923 & 3.543384 \\
\hline 4 & -2.796043 & -1.622033 & 1.433542 \\
\hline
\end{tabular}




$\begin{array}{lrrr}\mathrm{C} & -4.578043 & 0.328148 & 4.267090 \\ \mathrm{H} & -5.806263 & 1.166404 & 2.709319 \\ \mathrm{C} & -3.533351 & -0.545673 & 4.569883 \\ \mathrm{H} & -2.082975 & -1.925679 & 3.760510 \\ \mathrm{H} & -5.077893 & 0.879540 & 5.058918 \\ \mathrm{H} & -3.212331 & -0.676497 & 5.599719 \\ \mathrm{H} & -2.175471 & 3.060967 & -0.953755 \\ 1 & \text { imaginary frequency } & \\ \mathrm{E}_{\text {chloroform }}=-3563.766526 & \\ \mathrm{G}_{\text {chloroform }}=-3562.933194 & \end{array}$

\section{0-up}

$\begin{array}{lrrr}\text { C } & -0.834209 & -5.493939 & 3.071868 \\ \mathrm{C} & -0.048948 & -4.402425 & 3.799262 \\ \mathrm{C} & 0.531209 & -3.400767 & 2.798033 \\ \mathrm{C} & -0.478508 & -2.910341 & 1.772053 \\ \mathrm{C} & -1.642989 & -3.650977 & 1.513568 \\ \mathrm{C} & -2.020844 & -4.870672 & 2.334597 \\ \mathrm{C} & -0.206542 & -1.764556 & 0.989281 \\ \mathrm{C} & -1.029595 & -1.459098 & -0.100371 \\ \mathrm{C} & -2.216754 & -2.159621 & -0.349170 \\ \mathrm{C} & -2.497066 & -3.242323 & 0.488459 \\ \mathrm{C} & 0.985397 & -0.919224 & 1.285018 \\ \mathrm{C} & 1.146539 & -0.244617 & 2.516458 \\ \mathrm{C} & 2.380165 & 0.369114 & 2.793775 \\ \mathrm{C} & 3.403620 & 0.332612 & 1.838880 \\ \mathrm{C} & 3.228646 & -0.221672 & 0.569773 \\ \mathrm{C} & 1.989355 & -0.822515 & 0.322097 \\ \mathrm{C} & -0.029495 & -0.102949 & 3.467198 \\ \mathrm{C} & 0.376147 & 0.306064 & 4.885135 \\ \mathrm{C} & 1.367094 & 1.470641 & 4.851049 \\ \mathrm{C} & 2.645619 & 1.054141 & 4.121749 \\ \mathrm{O} & -0.666187 & -0.388417 & -0.929965 \\ \mathrm{O} & 1.728050 & -1.332223 & -0.953342 \\ \mathrm{P} & 0.665924 & -0.510799 & -1.902329 \\ \mathrm{~S} & 0.517929 & -1.337722 & -3.635590 \\ \mathrm{~S} & 1.254271 & 1.498446 & -1.980858 \\ \mathrm{H} & -0.173735 & -5.998244 & 2.353212 \\ \mathrm{H} & -1.188898 & -6.258634 & 3.772332 \\ \mathrm{H} & -0.716597 & -3.879935 & 4.499207 \\ \mathrm{H} & 0.762872 & -4.833048 & 4.396595 \\ \mathrm{H} & 0.991035 & -2.565872 & 3.329004 \\ \mathrm{H} & 1.354158 & -3.880706 & 2.246295 \\ \mathrm{H} & -2.780964 & -4.573844 & 3.073077 \\ \mathrm{H} & -2.504676 & -5.609801 & 1.685256 \\ \mathrm{H} & -3.412462 & -3.800482 & 0.309874 \\ \mathrm{H} & 4.364751 & 0.783000 & 2.074127 \\ \mathrm{H} & -0.703477 & 0.655518 & 3.045395 \\ \mathrm{H} & -0.622138 & -1.020138 & 3.491920 \\ \mathrm{H} & -0.519763 & 0.573374 & 5.456894 \\ \mathrm{H} & 0.839349 & -0.548057 & 5.400070 \\ \mathrm{H} & 0.909286 & 2.321142 & 4.331351 \\ \mathrm{H} & 1.610154 & 1.809042 & 5.864782 \\ \mathrm{H} & 3.294531 & 1.923419 & 3.960503 \\ \mathrm{H} & 3.216391 & 0.365474 & 4.762734 \\ \mathrm{H} & -3.676995 & 0.695408 & 1.265688 \\ \mathrm{C} & 4.288934 & -0.129066 & -0.466082 \\ \mathrm{C} & 4.727684 & 1.120797 & -0.861202 \\ & & & \end{array}$




\begin{tabular}{|c|c|c|c|}
\hline$\sqrt{2}$ & 5.722445 & 1.269722 & -1.852300 \\
\hline C & 6.276261 & 0.160756 & -2.445604 \\
\hline $\mathrm{C}$ & 5.868546 & -1.143644 & -2.063565 \\
\hline & 6.440212 & -2.299877 & -2.657045 \\
\hline c & 6.051887 & -3.560223 & -2.268252 \\
\hline C & 5.075020 & -3.717467 & -1.257209 \\
\hline C & 4.499369 & -2.617437 & -0.664648 \\
\hline C & 4.864466 & -1.301399 & -1.053070 \\
\hline $\mathrm{H}$ & 7.195795 & -2.167257 & -3.427369 \\
\hline $\mathrm{H}$ & 6.495379 & -4.436865 & -2.731693 \\
\hline $\mathrm{H}$ & 4.777445 & -4.715309 & -0.947748 \\
\hline $\mathrm{H}$ & 3.756604 & -2.750497 & 0.113762 \\
\hline $\mathrm{H}$ & 7.034669 & 0.265004 & -3.217386 \\
\hline $\mathrm{H}$ & 6.033096 & 2.266648 & -2.151142 \\
\hline $\mathrm{H}$ & 4.265658 & 2.003366 & -0.431534 \\
\hline C & -3.207857 & -1.781849 & -1.391863 \\
\hline C & -2.923982 & -1.916736 & -2.735063 \\
\hline C & -3.888812 & -1.607824 & -3.720898 \\
\hline C & -5.134810 & -1.154487 & -3.356526 \\
\hline C & -5.464295 & -0.979156 & -1.986779 \\
\hline C & -6.725576 & -0.461613 & -1.587930 \\
\hline C & -7.028244 & -0.266481 & -0.260797 \\
\hline C & -6.078322 & -0.598783 & 0.733660 \\
\hline C & -4.850136 & -1.113301 & 0.380641 \\
\hline C & -4.496980 & -1.308734 & -0.983578 \\
\hline $\mathrm{H}$ & -1.946735 & -2.270701 & -3.039343 \\
\hline $\mathrm{H}$ & -3.632930 & -1.732849 & -4.768898 \\
\hline $\mathrm{H}$ & -5.880859 & -0.911766 & -4.109145 \\
\hline $\mathrm{H}$ & -7.449508 & -0.212732 & -2.360069 \\
\hline $\mathrm{H}$ & -7.993860 & 0.138393 & 0.027547 \\
\hline $\mathrm{H}$ & -6.320032 & -0.449664 & 1.782263 \\
\hline $\mathrm{H}$ & -4.135106 & -1.382087 & 1.151148 \\
\hline C & -1.833481 & 3.401160 & -0.583255 \\
\hline C & -2.522519 & 2.317860 & -0.183653 \\
\hline C & -2.506016 & 2.188818 & 1.276823 \\
\hline C & -1.804399 & 3.211320 & 1.814717 \\
\hline $\mathrm{C}$ & -1.304929 & 4.087774 & 0.670264 \\
\hline 0 & -3.152441 & 1.186160 & 1.925959 \\
\hline C & -1.609736 & 3.541608 & 3.257607 \\
\hline $\mathrm{H}$ & -1.772548 & 5.082331 & 0.758544 \\
\hline $\mathrm{H}$ & -1.963478 & 2.726 & 3.896380 \\
\hline $\mathrm{H}$ & -0.554549 & 3.7307 & 3.493866 \\
\hline $\mathrm{H}$ & -2.159875 & 4.449718 & 3.544382 \\
\hline 0 & -3.217721 & 1.443329 & -0.944860 \\
\hline C & -3.431198 & 1.934640 & -2.286713 \\
\hline C & -2.174337 & 2.573285 & -2.875544 \\
\hline $\mathrm{H}$ & -3.746137 & 1.065936 & -2.862223 \\
\hline $\mathrm{H}$ & -4.256644 & 2.659099 & -2.245532 \\
\hline C & -1.674769 & 3.761746 & -2.025745 \\
\hline $\mathrm{H}$ & -2.389977 & 2.889379 & -3.902583 \\
\hline $\mathrm{H}$ & -0.625227 & 3.973245 & -2.270336 \\
\hline $\mathrm{H}$ & -2.235681 & 4.676886 & -2.272814 \\
\hline $\mathrm{H}$ & 0.962777 & 1.813977 & -0.698585 \\
\hline C & 0.198546 & 4.293313 & 0.615775 \\
\hline C & 1.091967 & 3.371373 & 1.176731 \\
\hline C & 0.721963 & 5.406109 & -0.055375 \\
\hline C & 2.470738 & 3.571118 & 1.081620 \\
\hline $\mathrm{H}$ & 0.705606 & 2.499084 & 1.694596 \\
\hline
\end{tabular}




$\begin{array}{lrrr}\mathrm{C} & 2.098505 & 5.595475 & -0.170587 \\ \mathrm{H} & 0.038782 & 6.130662 & -0.492074 \\ \mathrm{C} & 2.980318 & 4.678631 & 0.404259 \\ \mathrm{H} & 3.142821 & 2.851995 & 1.534758 \\ \mathrm{H} & 2.481803 & 6.463209 & -0.700448 \\ \mathrm{H} & 4.053703 & 4.829455 & 0.330868 \\ \mathrm{H} & -1.393334 & 1.810451 & -2.926894 \\ 0 & \text { imaginary frequencies } & \\ \mathrm{E}_{\text {chloroform }}=-3563.779518 & \\ \mathrm{G}_{\text {chloroform }}=-3562.944374 & \end{array}$

\section{TS4}

\begin{tabular}{|c|c|c|c|}
\hline $\mathrm{C}$ & 5.658376 & -4.158337 & -1.550450 \\
\hline C & 6.222560 & -2.772667 & -1.150922 \\
\hline C & 5.201127 & -1.622543 & -1.337799 \\
\hline C & 3.771630 & -2.040075 & -1.079594 \\
\hline C & 3.352264 & -3.231086 & -1.695251 \\
\hline C & 4.397412 & -4.036003 & -2.426392 \\
\hline C & 2.849246 & -1.270026 & -0.347428 \\
\hline C & 1.504516 & -1.670543 & -0.337526 \\
\hline C & 1.073132 & -2.869766 & -0.91371 \\
\hline C & 2.028164 & -3.647979 & -1.57674 \\
\hline C & 3.249069 & -0.035557 & 0.38778 \\
\hline C & 4.197212 & -0.061739 & 1.435189 \\
\hline C & 4.588398 & 1.148567 & 2.033162 \\
\hline C & 4.007884 & 2.347283 & 1.60973 \\
\hline C & 3.012218 & 2.395643 & 0.631797 \\
\hline C & 2.641828 & 1.177223 & 0.04961 \\
\hline C & 4.695794 & -1.386304 & 1.98737 \\
\hline C & 5.992560 & -1.263502 & 2.78959 \\
\hline C & 5.872976 & -0.139814 & 3.82029 \\
\hline C & 5.657722 & 1.198885 & 3.11053 \\
\hline $\mathrm{O}$ & 0.573139 & -0.833839 & 0.27872 \\
\hline $\mathrm{O}$ & 1.636162 & 1.169895 & -0.915248 \\
\hline$P$ & 0.149581 & 0.556990 & -0.545707 \\
\hline S & -0.844981 & 0.250832 & -2.188549 \\
\hline S & -0.634309 & 1.741975 & 0.92874 \\
\hline $\mathrm{H}$ & 6.423320 & -4.748685 & -2.06706 \\
\hline $\mathrm{H}$ & 5.383387 & -4.718546 & -0.649036 \\
\hline $\mathrm{H}$ & 6.554356 & -2.801951 & -0.10722 \\
\hline $\mathrm{H}$ & 7.111337 & -2.538055 & -1.747323 \\
\hline $\mathrm{H}$ & 5.482690 & -0.757964 & -0.73390 \\
\hline $\mathrm{H}$ & 5.250340 & -1.288219 & -2.384429 \\
\hline $\mathrm{H}$ & 4.007524 & -5.026611 & -2.68441 \\
\hline $\mathrm{H}$ & 4.660106 & -3.544027 & -3.37532 \\
\hline $\mathrm{H}$ & 1.715013 & -4.589426 & -2.02020 \\
\hline $\mathrm{H}$ & 4.324780 & 3.280975 & 2.0674 \\
\hline $\mathrm{H}$ & 3.907796 & -1.787610 & $2.6435 \varepsilon$ \\
\hline $\mathrm{H}$ & 4.809039 & -2.124178 & 1.19324 \\
\hline $\mathrm{H}$ & 6.216653 & -2.220106 & 3.27607 \\
\hline $\mathrm{H}$ & 6.829242 & -1.047597 & 2.10955 \\
\hline $\mathrm{H}$ & 5.022239 & -0.349361 & 4.48338 \\
\hline $\mathrm{H}$ & 6.766432 & -0.087490 & 4.45333 \\
\hline $\mathrm{H}$ & 5.408644 & 1.983089 & 3.83552 \\
\hline $\mathrm{H}$ & 6.606242 & 1.509085 & 2.64630 \\
\hline $\mathrm{H}$ & -3.029615 & -0.029826 & 3.58526 \\
\hline C & 2.351013 & 3.680905 & 0.28934 \\
\hline C & 1.739819 & 4.397227 & $1.3020 \varepsilon$ \\
\hline
\end{tabular}




\begin{tabular}{|c|c|c|c|}
\hline C & 1.079980 & 5.618661 & 1.046021 \\
\hline C & 1.034555 & 6.122221 & -0.231778 \\
\hline C & 1.662684 & 5.434500 & -1.302072 \\
\hline & 1.642617 & 5.951981 & -2.624235 \\
\hline & 2.274622 & 5.292559 & -3.651815 \\
\hline C & 2.964970 & 4.084540 & -3.396081 \\
\hline C & 2.999222 & 3.554364 & -2.127217 \\
\hline & 2.342245 & 4.198632 & -1.045788 \\
\hline $\mathrm{H}$ & 1.117729 & 6.886565 & -2.806889 \\
\hline $\mathrm{H}$ & 2.250425 & 5.698094 & -4.659424 \\
\hline $\mathrm{H}$ & 3.470110 & 3.571157 & -4.209255 \\
\hline & 3.534420 & 2.630473 & -1.942919 \\
\hline & 0.519458 & 7.056195 & -0.442673 \\
\hline $\mathrm{H}$ & 0.597361 & 6.146893 & 1.863339 \\
\hline $\mathrm{H}$ & 1.738576 & 3.986741 & 2.306989 \\
\hline C & -0.338090 & -3.336377 & -0.823620 \\
\hline & -1.145867 & -3.300402 & -1.942267 \\
\hline C & -2.482707 & -3.756275 & -1.895305 \\
\hline C & -3.002326 & -4.265147 & -0.729722 \\
\hline & -2.205091 & -4.338342 & 0.441580 \\
\hline C & -2.724740 & -4.839877 & 1.664073 \\
\hline C & -1.955268 & -4.8 & 2.804372 \\
\hline C & -0.621011 & -4.394825 & 2.767703 \\
\hline C & -0.083677 & -3.910336 & 1.596340 \\
\hline C & -0.853491 & -3.860687 & 0.403845 \\
\hline $\mathrm{H}$ & -0.75 & -2.878866 & -2.8 \\
\hline $\mathrm{H}$ & -3.103659 & -3.682720 & -2.781395 \\
\hline $\mathrm{H}$ & -4.037015 & -4.593880 & -0.684530 \\
\hline $\mathrm{H}$ & -3.751040 & -5.198987 & 1.681692 \\
\hline $\mathrm{H}$ & -2.367161 & -5.249453 & 3.733652 \\
\hline $\mathrm{H}$ & -0.016664 & -4.420560 & 3.670298 \\
\hline $\mathrm{H}$ & 0.940176 & -3.553105 & 1.572452 \\
\hline C & -4.816921 & 1.779080 & 0.282886 \\
\hline C & -4.75794 & 1.8727 & 1.636189 \\
\hline C & -3.870432 & 0.850185 & 2.145467 \\
\hline C & -3.242631 & 0.178055 & 1.094416 \\
\hline C & -3.947636 & 0.643677 & -0.190098 \\
\hline 0 & -3.6 & 0.7 & 3.452949 \\
\hline C & -2.644247 & -1.201835 & 1.215957 \\
\hline $\mathrm{H}$ & -3.202673 & 1.007014 & -0.910132 \\
\hline $\mathrm{H}$ & -1.924790 & -1.272446 & 2.040718 \\
\hline $\mathrm{H}$ & -3.4096 & -1.971 & 1.351589 \\
\hline $\mathrm{H}$ & -2.092359 & -1.444137 & 0.307836 \\
\hline 0 & -5.392066 & 2.766079 & 2.430605 \\
\hline C & -5.952172 & 3.865486 & 1.681055 \\
\hline C & -6.656918 & 3.405418 & 0.403108 \\
\hline $\mathrm{H}$ & -6.641056 & 4.3574 & 2.370239 \\
\hline $\mathrm{H}$ & -5.136061 & 4.559906 & 1.438577 \\
\hline C & -5.671495 & 2.685739 & -0.538570 \\
\hline $\mathrm{H}$ & -7.108076 & 4.274754 & -0.087387 \\
\hline $\mathrm{H}$ & -6.210378 & 2.110566 & -1.302071 \\
\hline $\mathrm{H}$ & -5.050167 & 3.411412 & -1.083297 \\
\hline $\mathrm{H}$ & -2.051354 & 0.950480 & 1.040672 \\
\hline $\mathrm{H}$ & -7.470572 & 2.723182 & 0.676091 \\
\hline C & -4.742922 & -0.461874 & -0.869851 \\
\hline C & -5.705114 & -1.192745 & -0.162221 \\
\hline C & -4.522791 & -0.757876 & -2.216690 \\
\hline C & -6.423550 & -2.209819 & -0.786165 \\
\hline
\end{tabular}




$\begin{array}{llll}\mathrm{H} & -5.887141 & -0.962011 & 0.884346 \\ \mathrm{C} & -5.251687 & -1.768173 & -2.848186 \\ \mathrm{H} & -3.763589 & -0.207549 & -2.763258 \\ \mathrm{C} & -6.199826 & -2.500168 & -2.135183 \\ \mathrm{H} & -7.163375 & -2.772334 & -0.222987 \\ \mathrm{H} & -5.070107 & -1.985649 & -3.897300 \\ \mathrm{H} & -6.763233 & -3.289977 & -2.624227 \\ \mathrm{1} & \text { imaginary frequency } & \\ \mathrm{E}_{\text {chloroform }}=-3563.765468 & \\ \mathrm{G}_{\text {chloroform }}=-3562.931942 & \end{array}$

\section{2-up-cis}

$\begin{array}{lrrr}\mathrm{C} & -0.984335 & -0.434590 & -0.616543 \\ \mathrm{C} & -1.870084 & 0.324834 & 0.060790 \\ \mathrm{C} & -1.427626 & 1.733909 & 0.135141 \\ \mathrm{C} & -0.158151 & 1.836929 & -0.723855 \\ \mathrm{C} & 0.230788 & 0.352005 & -1.048166 \\ \mathrm{O} & -1.960350 & 2.644434 & 0.739314 \\ \mathrm{C} & 0.904252 & 2.731289 & -0.090014 \\ \mathrm{H} & 0.381021 & 0.241800 & -2.130647 \\ \mathrm{H} & 0.470180 & 3.713938 & 0.115479 \\ \mathrm{H} & 1.765357 & 2.853910 & -0.754615 \\ \mathrm{H} & 1.266391 & 2.314886 & 0.853949 \\ \mathrm{O} & -3.023943 & -0.086117 & 0.634663 \\ \mathrm{C} & -3.112883 & -1.517807 & 0.752485 \\ \mathrm{C} & -2.673488 & -2.234572 & -0.524936 \\ \mathrm{H} & -2.485858 & -1.831480 & 1.599667 \\ \mathrm{H} & -4.157360 & -1.723152 & 0.995340 \\ \mathrm{C} & -1.209318 & -1.893690 & -0.859206 \\ \mathrm{H} & -3.325603 & -1.915630 & -1.346560 \\ \mathrm{H} & -0.515434 & -2.487225 & -0.246774 \\ \mathrm{H} & -0.979678 & -2.148338 & -1.902687 \\ \mathrm{H} & -0.480411 & 2.301817 & -1.666522 \\ \mathrm{C} & 1.502821 & -0.136681 & -0.371985 \\ \mathrm{C} & 1.524647 & -0.446996 & 0.993802 \\ \mathrm{C} & 2.686631 & -0.263390 & -1.105105 \\ \mathrm{C} & 2.702377 & -0.865389 & 1.610847 \\ \mathrm{H} & 0.610162 & -0.358527 & 1.573874 \\ \mathrm{C} & 3.867955 & -0.683148 & -0.492260 \\ \mathrm{H} & 2.682000 & -0.028829 & -2.167112 \\ \mathrm{C} & 3.879406 & -0.985346 & 0.869332 \\ \mathrm{H} & 2.700917 & -1.099467 & 2.671909 \\ \mathrm{H} & 4.777438 & -0.775950 & -1.079485 \\ \mathrm{H} & 4.796817 & -1.314163 & 1.349129 \\ \mathrm{H} & -2.809549 & -3.314262 & -0.398736 \\ \mathrm{O} & \text { imaginary frequencies } & \\ \mathrm{E} \text { chloroform } & =-731.579079 & \\ \mathrm{G} \mathrm{ch} 10 \mathrm{soform} & =-731.337551 & \\ & & & \end{array}$

\section{TS4'}

$\begin{array}{lrrr}\text { C } & 6.835060 & -1.438439 & -2.254110 \\ \text { C } & 6.887948 & -0.546532 & -1.012675 \\ \text { C } & 5.518596 & 0.085947 & -0.755034 \\ \text { C } & 4.374733 & -0.913980 & -0.789689 \\ \text { C } & 4.516987 & -2.139710 & -1.461499 \\ \text { C } & 5.852074 & -2.589565 & -2.028190 \\ \text { C } & 3.119443 & -0.577408 & -0.236879\end{array}$




\begin{tabular}{|c|c|c|c|}
\hline C & 2.020972 & -1.406700 & -0.483557 \\
\hline & 2.143816 & -2.647821 & -1.118630 \\
\hline C & 3.415511 & -2.992497 & -1.581656 \\
\hline C & 2.921863 & 0.642236 & 0.600620 \\
\hline & 3.584239 & 0.805638 & 1.838610 \\
\hline c & 3.388277 & 1.993091 & 2.564304 \\
\hline C & 2.516536 & 2.968533 & 2.071825 \\
\hline C & 1.785784 & 2.798901 & 0.894073 \\
\hline C & 2.004413 & 1.610195 & 0.186864 \\
\hline C & 4.391733 & -0.336488 & 2.434503 \\
\hline C & 5.343596 & 0.103018 & 3.549342 \\
\hline C & 4.611113 & 0.987407 & 4.559107 \\
\hline C & 4.116066 & 2.260620 & 3.869767 \\
\hline 0 & 0.760117 & -0.971281 & -0.076245 \\
\hline 0 & 1.271413 & 1.370457 & -0.977268 \\
\hline $\mathrm{P}$ & 0.059108 & 0.257883 & -0.949395 \\
\hline$S$ & -0.349536 & -0.218191 & -2.811511 \\
\hline $\mathrm{S}$ & -1.381069 & 0.933769 & 0.325263 \\
\hline $\mathrm{H}$ & 6.511657 & -0.838388 & -3.115690 \\
\hline $\mathrm{H}$ & 7.827104 & -1.837044 & -2.496093 \\
\hline $\mathrm{H}$ & 7.187125 & -1.149876 & -0.143438 \\
\hline $\mathrm{H}$ & 7.641380 & 0.241368 & -1.127049 \\
\hline $\mathrm{H}$ & 5.525346 & 0.641167 & 0.185287 \\
\hline $\mathrm{H}$ & 5.320534 & 0.838611 & -1.533348 \\
\hline $\mathrm{H}$ & 6.304362 & -3.309591 & -1.329288 \\
\hline $\mathrm{H}$ & 5.684742 & -3.141385 & -2.960938 \\
\hline $\mathrm{H}$ & 3.541585 & -3.955085 & -2.070755 \\
\hline $\mathrm{H}$ & 2.376432 & 3.887429 & 2.635321 \\
\hline $\mathrm{H}$ & 3.675875 & -1.066583 & 2.842889 \\
\hline $\mathrm{H}$ & 4.940610 & -0.876986 & 1.661295 \\
\hline $\mathrm{H}$ & 5.769849 & -0.781164 & 4.037410 \\
\hline $\mathrm{H}$ & 6.184396 & 0.664713 & 3.117629 \\
\hline $\mathrm{H}$ & 3.757055 & 0.433306 & 4.972512 \\
\hline $\mathrm{H}$ & 5.262589 & 1.247157 & 5.401602 \\
\hline $\mathrm{H}$ & 3.463694 & 2.833461 & 4.539640 \\
\hline $\mathrm{H}$ & 4.980492 & 2.909869 & 3.663213 \\
\hline $\mathrm{H}$ & -2.581945 & -0.235355 & -2.785998 \\
\hline C & 0.774233 & 3.803791 & 0.479040 \\
\hline C & -0.233491 & 4.12 & 1.369582 \\
\hline C & -1.245956 & 5.050501 & 1.032256 \\
\hline C & -1.246443 & 5.650897 & -0.203938 \\
\hline C & -0.222794 & 5.368396 & -1.144777 \\
\hline C & -0.197619 & 5.996013 & -2.418214 \\
\hline C & 0.811377 & 5.736027 & -3.315079 \\
\hline C & 1.849016 & 4.838654 & -2.969546 \\
\hline C & 1.850791 & 4.210226 & -1.745935 \\
\hline C & 0.814473 & 4.440021 & -0.802902 \\
\hline $\mathrm{H}$ & -0.995002 & 6.691200 & -2.669150 \\
\hline $\mathrm{H}$ & 0.817847 & 6.220655 & -4.287313 \\
\hline $\mathrm{H}$ & 2.649073 & 4.643297 & -3.677846 \\
\hline $\mathrm{H}$ & 2.653236 & 3.528108 & -1.490804 \\
\hline $\mathrm{H}$ & -2.029345 & 6.352816 & -0.480121 \\
\hline $\mathrm{H}$ & -2.031546 & 5.268757 & 1.750115 \\
\hline $\mathrm{H}$ & -0.264568 & 3.623156 & 2.330803 \\
\hline C & 0.998189 & -3.571703 & -1.320254 \\
\hline C & & -3.975411 & -2.605157 \\
\hline C & -0.360839 & -4.888844 & -2.857698 \\
\hline C & -1.096573 & -5.400881 & -1.815807 \\
\hline
\end{tabular}




\begin{tabular}{lrrr}
$\mathrm{H}$ & 0.337584 & 0.768347 & -1.758291 \\
$\mathrm{H}$ & -0.561131 & 3.961304 & -0.202341 \\
$\mathrm{H}$ & 0.927288 & 3.367634 & -0.978749 \\
$\mathrm{H}$ & -0.656814 & 3.037723 & -1.710306 \\
$\mathrm{O}$ & -3.057346 & -0.505449 & 0.538221 \\
$\mathrm{C}$ & -2.988232 & -1.938338 & 0.423542 \\
$\mathrm{C}$ & -2.312622 & -2.387807 & -0.872592 \\
$\mathrm{H}$ & -2.437155 & -2.325300 & 1.292933 \\
$\mathrm{H}$ & -4.022633 & -2.282535 & 0.483636 \\
$\mathrm{C}$ & -0.869482 & -1.854581 & -0.942890 \\
$\mathrm{H}$ & -2.891356 & -2.002941 & -1.720517 \\
$\mathrm{H}$ & -0.189891 & -2.469556 & -0.336061 \\
$\mathrm{H}$ & -0.483575 & -1.910121 & -1.969496 \\
$\mathrm{H}$ & 0.351446 & 1.957603 & 0.979208 \\
$\mathrm{C}$ & 1.616444 & 0.058783 & -0.216324 \\
$\mathrm{C}$ & 1.764357 & -0.510809 & 1.055484 \\
$\mathrm{C}$ & 2.743629 & 0.174727 & -1.034234 \\
$\mathrm{C}$ & 3.010167 & -0.948597 & 1.499254 \\
$\mathrm{H}$ & 0.891092 & -0.611023 & 1.695929 \\
$\mathrm{C}$ & 3.994539 & -0.261144 & -0.593286 \\
$\mathrm{H}$ & 2.639987 & 0.611738 & -2.024711 \\
$\mathrm{C}$ & 4.131284 & -0.824524 & 0.674941 \\
$\mathrm{H}$ & 3.107514 & -1.388192 & 2.488191 \\
$\mathrm{H}$ & 4.860325 & -0.162071 & -1.242282 \\
$\mathrm{H}$ & 5.102979 & -1.166781 & 1.019383 \\
$\mathrm{H}$ & -2.334129 & -3.481578 & -0.930271 \\
$\mathrm{O}$ & imaginary frequencies & \\
$\mathrm{E}_{\text {chloroform }}=-731.580524$ & \\
$\mathrm{G}_{\text {chloroform }}=-731.339287$ & \\
\multicolumn{5}{c}{-10736} &
\end{tabular}

\section{TS5}

$\begin{array}{lrrr}\text { C } & -6.846157 & -0.053084 & -2.521759 \\ \text { C } & -6.674198 & -1.210317 & -1.536437 \\ \text { C } & -5.195524 & -1.395805 & -1.189163 \\ \text { C } & -4.500405 & -0.103474 & -0.794377 \\ \text { C } & -5.013028 & 1.138252 & -1.210607 \\ \text { C } & -6.370374 & 1.253449 & -1.881946 \\ \text { C } & -3.271009 & -0.135845 & -0.101407 \\ \text { C } & -2.540568 & 1.047067 & 0.039916 \\ \text { C } & -3.008119 & 2.293351 & -0.396597 \\ \text { C } & -4.273789 & 2.304128 & -0.990251 \\ \text { C } & -2.696199 & -1.395936 & 0.460051 \\ \text { C } & -3.360325 & -2.122262 & 1.474320 \\ \text { C } & -2.794221 & -3.322137 & 1.938217 \\ \text { C } & -1.566120 & -3.748900 & 1.426353 \\ \text { C } & -0.843718 & -3.009326 & 0.487437 \\ \text { C } & -1.435957 & -1.826789 & 0.025562 \\ \text { C } & -4.595998 & -1.547441 & 2.148933 \\ \text { C } & -5.416589 & -2.589132 & 2.912412 \\ \text { C } & -4.509516 & -3.420733 & 3.820180 \\ \text { C } & -3.490072 & -4.186929 & 2.974452 \\ \text { O } & -1.279004 & 0.961391 & 0.622231 \\ \text { O } & -0.732463 & -1.027439 & -0.877798 \\ \text { P } & -0.046722 & 0.369024 & -0.302475 \\ \text { S } & 0.430732 & 1.496986 & -1.816561 \\ \text { S } & 1.257870 & -0.091140 & 1.198744 \\ \text { H } & -6.257165 & -0.259052 & -3.426053 \\ \text { H } & -7.891648 & 0.044783 & -2.836412\end{array}$




\begin{tabular}{|c|c|c|c|}
\hline & -7.246047 & -0.998016 & -0.621583 \\
\hline & -7.074359 & -2.142319 & -1.952165 \\
\hline & -5.076882 & -2.158189 & -0.416677 \\
\hline & -4.669827 & -1.793992 & -2.070585 \\
\hline & -7.107724 & 1.566052 & -1.126906 \\
\hline & -6.342921 & 2.058632 & -2.625828 \\
\hline & -4.679512 & 3.256244 & -1.322610 \\
\hline & -1.134679 & -4.677948 & 1.789964 \\
\hline & -4.253577 & -0.775472 & 2.855160 \\
\hline & -5.227895 & -1.018971 & 1.433316 \\
\hline & -6.198819 & -2.086369 & 3.492935 \\
\hline & -5.926117 & -3.253739 & 2.199950 \\
\hline & -3.985747 & -2.752413 & 4.517491 \\
\hline & -5.094177 & -4.1 & 4.426989 \\
\hline & -2.738583 & -4.664239 & 3.614663 \\
\hline & -4.007571 & -5.007485 & 2.454489 \\
\hline & 2.371751 & 1.392696 & -3.353072 \\
\hline & 0.51 & -3.4 & 84789 \\
\hline & 1.479813 & -3.590015 & 1.069304 \\
\hline & 2.791501 & -4.015149 & 0.768475 \\
\hline & 3.132179 & -4.326592 & -0.526208 \\
\hline & 2.180 & -4.2 & -1.572557 \\
\hline & 2.524299 & -4.498566 & -2.919358 \\
\hline & 1.604157 & -4.356848 & -3.931485 \\
\hline & 0.288487 & -3.928591 & -3.634923 \\
\hline & -0.07 & -3. & -2.339826 \\
\hline & 0.852494 & -3.756946 & -1.272790 \\
\hline $\mathrm{H}$ & 3.536279 & -4.832832 & -3.135192 \\
\hline & 1.882036 & -4.576737 & -4.958424 \\
\hline & -0.436786 & -3.824759 & -4.4 \\
\hline $\mathrm{H}$ & -1.089141 & -3.319508 & -2.122584 \\
\hline & 4.141913 & -4.647773 & -0.769793 \\
\hline & 3.532084 & -4.070044 & 1.559780 \\
\hline F & 1.225605 & -3.3 & 2.089731 \\
\hline C & -2.163619 & 3.515916 & -0.372484 \\
\hline C & -1.993487 & 4.201291 & -1.563168 \\
\hline C & -1.138727 & 5.318715 & -1.660423 \\
\hline C & -0.435 & & -0.559601 \\
\hline C & -0.586589 & 5.090181 & 0.689282 \\
\hline C & 0.133151 & 5.520772 & 1.836621 \\
\hline C & -0.030528 & 4.896466 & 3.050986 \\
\hline C & -0.946167 & & 73446 \\
\hline C & -1.655359 & & 2.080597 \\
\hline C & -1.481332 & 3.974766 & 0.801257 \\
\hline $\mathrm{H}$ & -2.487412 & 3.830499 & -2.455430 \\
\hline $\mathrm{H}$ & -1.019351 & 5.8 & -2.617121 \\
\hline $\mathrm{H}$ & 0.245248 & & -0.628436 \\
\hline $\mathrm{H}$ & 0.810647 & 6.365706 & 1.735518 \\
\hline $\mathrm{H}$ & 0.525927 & 5.234727 & 3.920760 \\
\hline $\mathrm{H}$ & -1.090221 & & 4.138507 \\
\hline $\mathrm{H}$ & -2.358470 & & 2.190184 \\
\hline C & 4.275415 & 1.847434 & 0.247292 \\
\hline C & 3.879623 & 2.431757 & -1.080875 \\
\hline C & 3.695011 & 1.438746 & -2.004305 \\
\hline C & 6054 & 0.171592 & -1.358681 \\
\hline C & 4.050276 & 0.337766 & 0.025665 \\
\hline 0 & 3.326554 & 1.601598 & -3.308734 \\
\hline C & 3.561526 & -1.103373 & -2.075351 \\
\hline
\end{tabular}




\begin{tabular}{|c|c|c|c|}
\hline $\mathrm{H}$ & 2.637024 & 0.132365 & 0.421347 \\
\hline $\mathrm{H}$ & 4.138514 & -1.162118 & -3.004897 \\
\hline $\mathrm{H}$ & 3.757038 & -1.976374 & -1.457151 \\
\hline $\mathrm{H}$ & 2.501432 & -1.131428 & -2.363913 \\
\hline 0 & 3.678239 & 3.732904 & -1.292608 \\
\hline C & 3.096915 & 4.467911 & -0.178804 \\
\hline C & 3.709562 & 4.096738 & 1.164503 \\
\hline $\mathrm{H}$ & 3.258552 & 5.517714 & -0.430275 \\
\hline $\mathrm{H}$ & 2.021425 & 4.261573 & -0.185762 \\
\hline C & 3.592126 & 2.586381 & 1.409696 \\
\hline $\mathrm{H}$ & 3.173652 & 4.649716 & 1.944414 \\
\hline $\mathrm{H}$ & 4.053035 & 2.306802 & 2.362207 \\
\hline $\mathrm{H}$ & 2.535827 & 2.308256 & 1.461798 \\
\hline $\mathrm{H}$ & 5.362871 & 1.988298 & 0.352304 \\
\hline C & 4.708567 & -0.671053 & 0.899514 \\
\hline C & 5.657393 & -1.575514 & 0.395306 \\
\hline C & 4.387486 & -0.745056 & 2.265229 \\
\hline C & 6.242999 & -2.536043 & 1.217384 \\
\hline $\mathrm{H}$ & 5.945786 & -1.514378 & -0.649304 \\
\hline C & 4.977829 & -1.700279 & 3.090980 \\
\hline $\mathrm{H}$ & 3.660500 & -0.056416 & 2.683681 \\
\hline C & 5.903422 & -2.605608 & 2.569792 \\
\hline $\mathrm{H}$ & 6.974707 & -3.224246 & 0.802788 \\
\hline $\mathrm{H}$ & 4.708988 & -1.739903 & 4.142916 \\
\hline $\mathrm{H}$ & 6.362462 & -3.351521 & 3.212465 \\
\hline $\mathrm{H}$ & 4.760657 & 4.412639 & 1.193741 \\
\hline \multicolumn{4}{|c|}{1 imaginary frequency } \\
\hline \multicolumn{4}{|c|}{$E_{\text {chloroform }}=-3563.759341$} \\
\hline & form $=-356$ & .926841 & \\
\hline
\end{tabular}

\section{TS6}

$\begin{array}{lrrr}\text { C } & 5.070440 & 4.838338 & 0.039499 \\ \mathrm{C} & 4.085677 & 4.934750 & 1.205552 \\ \mathrm{C} & 2.797087 & 4.176156 & 0.879345 \\ \mathrm{C} & 3.037754 & 2.779169 & 0.331214 \\ \mathrm{C} & 4.278592 & 2.432970 & -0.228321 \\ \mathrm{C} & 5.466619 & 3.377542 & -0.185858 \\ \mathrm{C} & 1.981472 & 1.841979 & 0.286676 \\ \mathrm{C} & 2.158981 & 0.639842 & -0.403267 \\ \mathrm{C} & 3.399919 & 0.256427 & -0.923374 \\ \mathrm{C} & 4.445497 & 1.171046 & -0.810395 \\ \mathrm{C} & 0.664166 & 2.103331 & 0.938301 \\ \mathrm{C} & 0.546607 & 2.259310 & 2.335917 \\ \mathrm{C} & -0.699807 & 2.619334 & 2.875317 \\ \mathrm{C} & -1.796379 & 2.774211 & 2.024293 \\ \mathrm{C} & -0.480958 & 2.147210 & 0.131868 \\ \mathrm{C} & 1.715390 & 1.917154 & 3.244543 \\ \mathrm{C} & 1.572183 & 2.472763 & 4.663293 \\ \mathrm{C} & 0.179139 & 2.170236 & 5.218127 \\ \mathrm{C} & -0.882112 & 2.865751 & 4.362247 \\ \mathrm{O} & 1.063352 & -0.210244 & -0.525554 \\ \mathrm{O} & -0.335777 & 1.840981 & -1.203327 \\ \mathrm{P} & -0.137594 & 0.243502 & -1.697525 \\ \mathrm{~S} & 0.590515 & 0.283480 & -3.501765 \\ \mathrm{~S} & -1.759627 & -0.802065 & -1.222626 \\ \mathrm{H} & 4.596485 & 5.237916 & -0.867534 \\ \mathrm{H} & 5.964824 & 5.444001 & 0.227123 \\ \mathrm{H} & 4.548583 & 4.507812 & 2.107057\end{array}$




\begin{tabular}{|c|c|c|c|}
\hline $\mathrm{H}$ & 3.848799 & 5.980264 & 1.435099 \\
\hline & 2.145212 & 4.144302 & 1.755246 \\
\hline $\mathrm{H}$ & 2.232346 & 4.737711 & 0.119729 \\
\hline $\mathrm{H}$ & 6.138840 & 3.063240 & 0.627287 \\
\hline & 6.046137 & 3.274488 & -1.111102 \\
\hline & 5.419807 & 0.892996 & -1.204768 \\
\hline $\mathrm{H}$ & -2.749671 & 3.081162 & 2.447160 \\
\hline $\mathrm{H}$ & 1.771075 & 0.819047 & 3.293206 \\
\hline & 2.661608 & 2.238966 & 2.804135 \\
\hline $\mathrm{H}$ & 2.351333 & 2.046009 & 5.305616 \\
\hline $\mathrm{H}$ & 1.728146 & 3.561065 & 4.653982 \\
\hline $\mathrm{H}$ & 0.014166 & 1.083199 & 5.204614 \\
\hline $\mathrm{H}$ & 0.090787 & 2.494224 & 6.261818 \\
\hline $\mathrm{H}$ & -1.888881 & 2.552402 & 4.665013 \\
\hline $\mathrm{H}$ & -0.834263 & 3.948612 & 4.553157 \\
\hline $\mathrm{H}$ & 1.078723 & -0.823165 & 1.170763 \\
\hline C & 3.632705 & -1.095004 & -1.504925 \\
\hline C & 3.687817 & -1.274312 & -2.871047 \\
\hline C & 3.944795 & -2.547917 & -3.428580 \\
\hline C & 4.156346 & -3.634578 & -2.613313 \\
\hline C & 4.115982 & -3.495694 & -1.201270 \\
\hline C & 4.328666 & -4.601174 & -0.335112 \\
\hline C & 4.270782 & -4.452300 & 1.031766 \\
\hline C & 4.011749 & -3.180756 & 1.593938 \\
\hline C & 3.814417 & -2.087656 & 0.782535 \\
\hline $\mathrm{C}$ & 3.847594 & -2.207971 & -0.632000 \\
\hline $\mathrm{H}$ & 3.499160 & -0.42 & -3.520157 \\
\hline $\mathrm{H}$ & 3.969540 & -2.659437 & -4.508711 \\
\hline $\mathrm{H}$ & 4.359990 & -4.615448 & -3.037359 \\
\hline $\mathrm{H}$ & 4.543688 & -5.572350 & -0.776431 \\
\hline $\mathrm{H}$ & 4.433652 & -5.30 & 1.681927 \\
\hline $\mathrm{H}$ & 3.966180 & -3.066562 & 2.673111 \\
\hline $\mathrm{H}$ & 3.624366 & -1.115143 & 1.222068 \\
\hline C & -1.375875 & -3.769926 & 0.259566 \\
\hline C & -0.027274 & -3.226789 & 0.618488 \\
\hline C & -0.137661 & -2.189918 & 1.489106 \\
\hline C & -1.558438 & -2.009988 & 1.816545 \\
\hline C & -2.345934 & -2.950331 & 1.058095 \\
\hline 0 & 0.814884 & -1.349215 & 1.960852 \\
\hline C & -2.032996 & -0.744690 & 2.459526 \\
\hline $\mathrm{H}$ & -1.887229 & -3.003591 & 2.486485 \\
\hline $\mathrm{H}$ & -3.054426 & -0.823925 & 2.837871 \\
\hline $\mathrm{H}$ & -1.352098 & -0.446937 & 3.258422 \\
\hline $\mathrm{H}$ & -1.995767 & 0.0 & 1.684580 \\
\hline 0 & 1.076467 & -3.724730 & 0.063936 \\
\hline C & 0.809020 & -4.698392 & -0.966929 \\
\hline C & -0.070808 & -5.823 & -0.422358 \\
\hline $\mathrm{H}$ & 0.345685 & -4.182584 & -1.817337 \\
\hline $\mathrm{H}$ & 1.790326 & -5.058368 & -1.268663 \\
\hline C & -1.415377 & -5.306052 & 0.165485 \\
\hline $\mathrm{H}$ & 0.497498 & -6.341518 & 0.356837 \\
\hline $\mathrm{H}$ & -2.263676 & -5.612388 & -0.451791 \\
\hline $\mathrm{H}$ & -1.568645 & -5.733012 & 1.161598 \\
\hline $\mathrm{H}$ & -1.588005 & -3.334185 & -0.740264 \\
\hline C & -3.803147 & -3.007043 & 0.950181 \\
\hline C & -4.523499 & -1.839266 & 0.637542 \\
\hline C & -4.487957 & -4.228259 & 1.082624 \\
\hline C & -5.903089 & -1.901924 & 0.463507 \\
\hline
\end{tabular}




\begin{tabular}{lllr}
$\mathrm{H}$ & -3.993338 & -0.909936 & 0.465034 \\
$\mathrm{C}$ & -5.869846 & -4.276516 & 0.929689 \\
$\mathrm{H}$ & -3.938238 & -5.131109 & 1.327223 \\
$\mathrm{C}$ & -6.578691 & -3.113557 & 0.617171 \\
$\mathrm{H}$ & -6.444329 & -1.002339 & 0.189507 \\
$\mathrm{H}$ & -6.393747 & -5.220186 & 1.047672 \\
$\mathrm{H}$ & -7.655640 & -3.155857 & 0.483125 \\
$\mathrm{H}$ & -0.253471 & -6.545928 & -1.224139 \\
$\mathrm{C}$ & -1.737314 & 2.510215 & 0.650480 \\
$\mathrm{C}$ & -2.984917 & 2.541952 & -0.154712 \\
$\mathrm{C}$ & -4.099160 & 1.887870 & 0.346061 \\
$\mathrm{C}$ & -3.092153 & 3.243008 & -1.400070 \\
$\mathrm{C}$ & -5.319624 & 1.849342 & -0.359312 \\
$\mathrm{H}$ & -4.020750 & 1.371081 & 1.297623 \\
$\mathrm{C}$ & -4.326535 & 3.175069 & -2.128075 \\
$\mathrm{C}$ & -5.425173 & 2.462048 & -1.584928 \\
$\mathrm{H}$ & -6.172007 & 1.331110 & 0.072224 \\
$\mathrm{H}$ & -6.353879 & 2.424007 & -2.148623 \\
$\mathrm{C}$ & -2.044315 & 4.039720 & -1.932783 \\
$\mathrm{C}$ & -4.437550 & 3.850830 & -3.372169 \\
$\mathrm{C}$ & -3.390845 & 4.587944 & -3.871511 \\
$\mathrm{C}$ & -2.187660 & 4.691428 & -3.135205 \\
$\mathrm{H}$ & -1.367611 & 5.287095 & -3.525332 \\
$\mathrm{H}$ & -1.118172 & 4.132443 & -1.381210 \\
$\mathrm{H}$ & -3.486401 & 5.098602 & -4.825597 \\
$\mathrm{H}$ & -5.374230 & 3.777741 & -3.919315 \\
$\mathrm{I}$ & imaginary frequency & \\
$\mathrm{E}_{\text {chloroform }}=-3563.724139$ & \\
$\mathrm{G}_{\mathrm{chloroform}}=-3562.890222$ & \\
\multicolumn{5}{c}{$=$} & &
\end{tabular}

\section{TS7}

$\begin{array}{lrrr}\text { C } & -0.397955 & 6.590658 & -0.814928 \\ \text { C } & -1.322047 & 6.040912 & 0.272706 \\ \text { C } & -1.590301 & 4.553027 & 0.037595 \\ \text { C } & -0.328212 & 3.740241 & -0.204348 \\ \text { C } & 0.841929 & 4.368592 & -0.661791 \\ \text { C } & 0.956183 & 5.879906 & -0.757917 \\ \mathrm{C} & -0.352263 & 2.332602 & -0.076221 \\ \mathrm{C} & 0.727747 & 1.583811 & -0.563558 \\ \mathrm{C} & 1.912901 & 2.193517 & -0.993164 \\ \mathrm{C} & 1.949565 & 3.587978 & -1.004785 \\ \mathrm{C} & -1.503593 & 1.630005 & 0.564167 \\ \mathrm{C} & -1.869050 & 1.868292 & 1.911531 \\ \mathrm{C} & -3.007208 & 1.223911 & 2.428628 \\ \mathrm{C} & -3.742790 & 0.356086 & 1.614321 \\ \mathrm{C} & -3.359271 & 0.041798 & 0.309785 \\ \mathrm{C} & -2.212506 & 0.682804 & -0.179757 \\ \mathrm{C} & -0.984429 & 2.717621 & 2.812480 \\ \mathrm{C} & -1.663650 & 3.151300 & 4.113733 \\ \mathrm{C} & -2.366037 & 1.964113 & 4.773229 \\ \mathrm{C} & -3.476771 & 1.455410 & 3.853611 \\ \mathrm{O} & 0.645883 & 0.196751 & -0.566770 \\ \mathrm{O} & -1.755932 & 0.379777 & -1.453368 \\ \mathrm{P} & -0.406170 & -0.576806 & -1.615040 \\ \mathrm{~S} & 0.080413 & -0.597571 & -3.496428 \\ \mathrm{~S} & -0.706053 & -2.348965 & -0.663995 \\ \mathrm{H} & -0.861294 & 6.426957 & -1.797452 \\ \mathrm{H} & -0.256837 & 7.672027 & -0.703906\end{array}$




\begin{tabular}{|c|c|c|c|}
\hline & -0.851915 & 6.182629 & 1.256743 \\
\hline & -2.272255 & 6.587020 & 0.293152 \\
\hline & -2.177098 & 4.136447 & 0.858297 \\
\hline & -2.224333 & 4.442527 & -0.855374 \\
\hline & 1.510775 & 6.245187 & 0.120029 \\
\hline & 1.566400 & 6.144798 & -1.629563 \\
\hline & 2.865173 & 4.077610 & -1.327390 \\
\hline & -4.631900 & -0.122252 & 2.016741 \\
\hline & -0.091975 & 2.124554 & 3.059273 \\
\hline & -0.605342 & 3.590831 & 2.278668 \\
\hline & -0.917590 & 3.591282 & 4.785561 \\
\hline & -2.403936 & 3.936222 & 3.902967 \\
\hline & -1.636517 & 1.161649 & 4.951872 \\
\hline & -2.782743 & 2.242939 & 5.747916 \\
\hline & -3.912695 & 0.529940 & 4.249408 \\
\hline & -4.294666 & 2.191624 & 3.844133 \\
\hline & 4.593629 & -0.997733 & 1.507315 \\
\hline & -4.110878 & -0.966989 & -0.479239 \\
\hline & -4.254061 & -2.240219 & 0.040585 \\
\hline & -4.947170 & -3.246832 & -0.665559 \\
\hline C & -5.497761 & -2.973727 & -1.894795 \\
\hline C & -5.392172 & -1.677500 & -2.461737 \\
\hline & -5.971103 & -1.373907 & -3.722266 \\
\hline C & -5.883506 & -0.109714 & -4.255315 \\
\hline C & -5.215691 & 0.912356 & -3.540678 \\
\hline C & -4.640559 & 0.649781 & -2.319055 \\
\hline C & -4.696330 & -0.648433 & -1.746315 \\
\hline $\mathrm{H}$ & -6.488973 & -2.165458 & -4.258414 \\
\hline $\mathrm{H}$ & -6.328288 & 0.108966 & -5.222059 \\
\hline $\mathrm{H}$ & -5.154716 & 1.911649 & -3.962336 \\
\hline $\mathrm{H}$ & -4.135042 & 1.442432 & -1.780065 \\
\hline $\mathrm{H}$ & -6.022553 & -3.745581 & -2.452337 \\
\hline $\mathrm{H}$ & -5.025081 & -4.241482 & -0.235974 \\
\hline $\mathrm{H}$ & -3.786253 & -2.478135 & 0.989774 \\
\hline C & 3.117430 & 1.403337 & -1.372142 \\
\hline C & 3.185267 & 0.761759 & -2.591425 \\
\hline C & 4.336968 & 0.038302 & -2.973544 \\
\hline C & 5.413341 & -0.054142 & -2.123624 \\
\hline C & 5.378810 & 0.565399 & -0.846916 \\
\hline $\mathrm{C}$ & 6.458196 & 0.446681 & 0.069511 \\
\hline C & 6.421107 & 1.075182 & 1.295071 \\
\hline C & 5.297222 & 1.855964 & 1.655582 \\
\hline C & 4.226360 & 1.971865 & 0.799278 \\
\hline $\mathrm{C}$ & 4.225343 & 1.328828 & -0.468102 \\
\hline $\mathrm{H}$ & 2.336770 & 0.811490 & -3.262060 \\
\hline $\mathrm{H}$ & 4.363239 & -0.438004 & -3.949445 \\
\hline $\mathrm{H}$ & 6.311752 & -0.589820 & -2.420986 \\
\hline $\mathrm{H}$ & 7.323791 & -0.144087 & -0.220840 \\
\hline $\mathrm{H}$ & 7.256039 & 0.978808 & 1.983360 \\
\hline $\mathrm{H}$ & 5.272863 & 2.347723 & 2.623537 \\
\hline $\mathrm{H}$ & 3.355067 & 2.546327 & 1.092363 \\
\hline C & 2.821475 & -2.871936 & -0.914823 \\
\hline C & 3.418982 & -2.499577 & 0.229415 \\
\hline C & 2.826483 & -1.641472 & 1.261572 \\
\hline C & 1.500725 & -1.564515 & 1.655689 \\
\hline C & 0.603976 & -2.623913 & 1.330427 \\
\hline 0 & 3.708044 & -0.854083 & 1.896032 \\
\hline $\mathrm{C}$ & 1.084361 & -0.410238 & 2.531146 \\
\hline
\end{tabular}




\begin{tabular}{|c|c|c|c|}
\hline $\mathrm{H}$ & 0.704366 & -0.747608 & 3.501722 \\
\hline $\mathrm{H}$ & 1.921818 & 0.265957 & 2.700882 \\
\hline $\mathrm{H}$ & 0.288252 & 0.150867 & 2.037393 \\
\hline 0 & 4.737936 & -2.774620 & 0.538700 \\
\hline & 5.541350 & -3.262987 & -0.549274 \\
\hline & 4.801598 & -4.318317 & -1.362251 \\
\hline $\mathrm{H}$ & 5.808354 & -2.412551 & -1.184726 \\
\hline $\mathrm{H}$ & 6.444354 & -3.657602 & -0.078025 \\
\hline C & 3.520805 & -3.707682 & -1.943838 \\
\hline $\mathrm{H}$ & 4.553943 & -5.163085 & -0.708543 \\
\hline $\mathrm{H}$ & 3.748942 & -3.079932 & -2.817177 \\
\hline $\mathrm{H}$ & 2.842040 & -4.489400 & -2.306463 \\
\hline C & -0.544396 & -2.993500 & 2.186019 \\
\hline C & -1.491372 & -2.075111 & 2.670783 \\
\hline C & -0.692306 & -4.348365 & 2.523489 \\
\hline C & -2.529257 & -2.499740 & 3.492334 \\
\hline $\mathrm{H}$ & -1.451315 & -1.041060 & 2.360928 \\
\hline C & -1.732274 & -4.772673 & 3.349212 \\
\hline $\mathrm{H}$ & 0.019537 & -5.072705 & 2.136480 \\
\hline C & -2.652605 & -3.848006 & 3.841204 \\
\hline $\mathrm{H}$ & -3.257095 & -1.775267 & 3.845224 \\
\hline $\mathrm{H}$ & -1.825426 & -5.825033 & 3.601391 \\
\hline $\mathrm{H}$ & -3.468193 & -4.174848 & 4.479873 \\
\hline $\mathrm{H}$ & 5.458983 & -4.696206 & -2.153073 \\
\hline $\mathrm{H}$ & 1.082558 & -3.482552 & 0.872258 \\
\hline $\mathrm{H}$ & 1.807140 & -2.549263 & -1.109857 \\
\hline \multicolumn{4}{|c|}{ imaginary frequency } \\
\hline \multicolumn{4}{|c|}{$E_{\text {chloroform }}=-3563.756589$} \\
\hline \multicolumn{4}{|c|}{$\mathrm{G}_{\mathrm{chloroform}}=-3562.917397$} \\
\hline
\end{tabular}

\section{3}

\begin{tabular}{|c|c|c|c|}
\hline 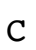 & -0.645353 & 6.675588 & -0.177959 \\
\hline & -1.522359 & 5.994176 & 0.873611 \\
\hline & -1.749939 & 4.526938 & 0.503910 \\
\hline & -0.469511 & 3.785541 & 0.155119 \\
\hline & 0.669280 & 4.492842 & -0.265627 \\
\hline & 0.731890 & 6.009458 & -0.221036 \\
\hline & -0.444537 & 2.372605 & 0.151527 \\
\hline & 0.654562 & 1.714891 & -0.412071 \\
\hline & 1.806127 & 2.396673 & -0.818200 \\
\hline & 1.793104 & 3.787146 & -0.706097 \\
\hline & -1.562152 & 1.570883 & 0.731879 \\
\hline & -1.893182 & 1.642604 & 2.106482 \\
\hline & -2.976189 & 0.884113 & 2.582807 \\
\hline & -3.714766 & 0.093804 & 1.694670 \\
\hline & -3.374094 & -0.043786 & 0.348193 \\
\hline & -2.257047 & 0.681548 & -0.089169 \\
\hline & -1.005072 & 2.416586 & 3.067575 \\
\hline 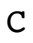 & -1.634105 & 2.641346 & 4.444754 \\
\hline - & -2.247926 & 1.344219 & 4.972190 \\
\hline & -3.381367 & 0.901959 & 4.045322 \\
\hline & 0.625318 & 0.324258 & -0.524588 \\
\hline & -1.790563 & 0.485104 & -1.388994 \\
\hline & -0.407184 & -0.397729 & -1.586623 \\
\hline & 0.071207 & -0.426437 & -3.458274 \\
\hline & -0.789232 & -2.233590 & -0.661834 \\
\hline & -1.129297 & 6.590895 & -1.160581 \\
\hline & -0.535460 & 7.745530 & 0.033033 \\
\hline
\end{tabular}




\begin{tabular}{lrrr}
$\mathrm{H}$ & -1.030645 & 6.057265 & 1.855118 \\
$\mathrm{H}$ & -2.488311 & 6.503312 & 0.968831 \\
$\mathrm{H}$ & -2.298192 & 4.013829 & 1.296358 \\
$\mathrm{H}$ & -2.406020 & 4.479477 & -0.378708 \\
$\mathrm{H}$ & 1.298107 & 6.308506 & 0.674190 \\
$\mathrm{H}$ & 1.309945 & 6.375802 & -1.077687 \\
$\mathrm{H}$ & 2.682083 & 4.336488 & -1.005687 \\
$\mathrm{H}$ & -4.572153 & -0.461006 & 2.067067 \\
$\mathrm{H}$ & -0.074344 & 1.843840 & 3.187281 \\
$\mathrm{H}$ & -0.699301 & 3.371542 & 2.635765 \\
$\mathrm{H}$ & -0.873647 & 3.026239 & 5.133827 \\
$\mathrm{H}$ & -2.417757 & 3.409215 & 4.374755 \\
$\mathrm{H}$ & -1.475095 & 0.564142 & 5.009956 \\
$\mathrm{H}$ & -2.626338 & 1.472744 & 5.992732 \\
$\mathrm{H}$ & -3.748166 & -0.091577 & 4.330221 \\
$\mathrm{H}$ & -4.233426 & 1.586831 & 4.171857 \\
$\mathrm{H}$ & 4.408632 & -0.889019 & 1.471896 \\
$\mathrm{C}$ & -4.131069 & -0.961782 & -0.539346 \\
$\mathrm{C}$ & -4.272640 & -2.285846 & -0.164818 \\
$\mathrm{C}$ & -4.969913 & -3.208544 & -0.974034 \\
$\mathrm{C}$ & -5.527642 & -2.800310 & -2.161811 \\
$\mathrm{C}$ & -5.427429 & -1.448401 & -2.580245 \\
$\mathrm{C}$ & -6.015988 & -1.007818 & -3.795059 \\
$\mathrm{C}$ & -5.936856 & 0.308528 & -4.183407 \\
$\mathrm{C}$ & -5.269083 & 1.246704 & -3.361940 \\
$\mathrm{C}$ & -4.683785 & 0.850936 & -2.181626 \\
$\mathrm{C}$ & -4.728537 & -0.504010 & -1.758627 \\
$\mathrm{H}$ & -6.535448 & -1.736305 & -4.412679 \\
$\mathrm{C}$ & 1.3777075 & -1.671644 & 1.323025 \\
$\mathrm{C}$ & 0.507622 & -2.711947 & 0.714440 \\
$\mathrm{O}$ & 3.501552 & -0.844684 & 1.821577 \\
$\mathrm{C}$ & 0.814665 & -0.756798 & 2.376009 \\
$\mathrm{H}$ & -5.389767 & 0.632614 & -5.116089 \\
$\mathrm{H}$ & -4.181803 & 2.288028 & -3.666979 \\
$\mathrm{H}$ & -1.582152 & -1.558918 \\
$\mathrm{H}$ & -6.054750 & -3.505854 & -2.799094 \\
$\mathrm{H}$ & -5.044878 & -4.244671 & -0.657245 \\
$\mathrm{H}$ & -3.799242 & -2.631631 & 0.747948 \\
$\mathrm{C}$ & 3.018177 & 1.681430 & -1.306752 \\
$\mathrm{C}$ & 3.073370 & 1.194954 & -2.595959 \\
$\mathrm{C}$ & 4.223713 & 0.530013 & -3.077025 \\
$\mathrm{C}$ & 5.311579 & 0.345413 & -2.258206 \\
$\mathrm{C}$ & 5.296363 & 0.818802 & -0.920050 \\
$\mathrm{C}$ & 6.397053 & 0.618658 & -0.045097 \\
$\mathrm{C}$ & 6.376267 & 1.103852 & 1.243981 \\
$\mathrm{C}$ & 5.247313 & 1.815768 & 1.713740 \\
$\mathrm{C}$ & 4.156451 & 2.008158 & 0.897213 \\
$\mathrm{C}$ & 4.141230 & 1.516010 & -0.435060 \\
$\mathrm{H}$ & 2.216626 & 1.319954 & -3.246354 \\
$\mathrm{H}$ & -.236902 & 0.166845 & -4.100506 \\
$\mathrm{H}$ & 7.265457 & -0.157929 & -2.625161 \\
\hline
\end{tabular}




\begin{tabular}{|c|c|c|c|}
\hline $\mathrm{H}$ & 0.674952 & -1.267075 & 3.337409 \\
\hline $\mathrm{H}$ & 1.485326 & 0.089357 & 2.535517 \\
\hline $\mathrm{H}$ & -0.158821 & -0.371583 & 2.065986 \\
\hline 0 & 4.682999 & -2.653437 & 0.333329 \\
\hline C & 5.587613 & -2.991539 & -0.727503 \\
\hline C & 4.931713 & -3.914216 & -1.747900 \\
\hline $\mathrm{H}$ & 5.915890 & -2.064102 & -1.210411 \\
\hline $\mathrm{H}$ & 6.443430 & -3.458314 & -0.233819 \\
\hline C & 3.724059 & -3.202918 & -2.370459 \\
\hline $\mathrm{H}$ & 4.609896 & -4.830682 & -1.238837 \\
\hline $\mathrm{H}$ & 4.051458 & -2.485534 & -3.136351 \\
\hline $\mathrm{H}$ & 3.076334 & -3.921993 & -2.887937 \\
\hline C & -0.344073 & -3.453807 & 1.728802 \\
\hline C & -1.480336 & -2.889029 & 2.325317 \\
\hline C & 0.049481 & -4.739527 & 2.113883 \\
\hline $\mathrm{C}$ & -2.195494 & -3.592079 & 3.291874 \\
\hline $\mathrm{H}$ & -1.813571 & -1.903906 & 2.021484 \\
\hline C & -0.667591 & -5.446122 & 3.080639 \\
\hline $\mathrm{H}$ & 0.928246 & -5.186840 & 1.656493 \\
\hline C & -1.792807 & -4.874105 & 3.673370 \\
\hline $\mathrm{H}$ & -3.073134 & -3.137363 & 3.743894 \\
\hline $\mathrm{H}$ & -0.348528 & -6.444888 & 3.365096 \\
\hline $\mathrm{H}$ & -2.355096 & -5.423849 & 4.422870 \\
\hline $\mathrm{H}$ & 5.664745 & -4.201221 & -2.510354 \\
\hline $\mathrm{H}$ & 1.125692 & -3.426578 & 0.175889 \\
\hline $\mathrm{H}$ & 1.974466 & -2.076298 & -1.578379 \\
\hline \multicolumn{4}{|c|}{0 imaginary frequencies } \\
\hline \multicolumn{4}{|c|}{$E_{\text {chloroform }}=-3563.767736$} \\
\hline \multicolumn{4}{|c|}{$\mathrm{G}_{\mathrm{chloroform}}=-3562.928515$} \\
\hline
\end{tabular}

14

\begin{tabular}{|c|c|c|c|}
\hline 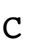 & 6.271555 & -1.656456 & -2.847044 \\
\hline & 6.503507 & -0.671717 & -1.700000 \\
\hline & 5.176056 & -0.058796 & -1.248142 \\
\hline & 4.084547 & -1.089170 & -1.011375 \\
\hline & 4.153685 & -2.355803 & -1.615407 \\
\hline & 5.388760 & -2.812702 & -2.371350 \\
\hline & 2.933439 & -0.753427 & -0.266532 \\
\hline & 1.846978 & -1.630849 & -0.250838 \\
\hline & 1.885441 & -2.898311 & -0.843476 \\
\hline & 3.075144 & -3.235926 & -1.497767 \\
\hline & 2.825957 & 0.530577 & 0.484508 \\
\hline & 3.689237 & 0.827615 & 1.562461 \\
\hline & 3.585560 & 2.079618 & 2.191066 \\
\hline & 2.620393 & 2.990513 & 1.755107 \\
\hline & 1.700028 & 2.695090 & 0.745510 \\
\hline$C$ & 1.815878 & 1.436061 & 0.143786 \\
\hline$C$ & 4.628263 & -0.234731 & 2.110719 \\
\hline 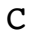 & 5.746898 & 0.333064 & 2.987225 \\
\hline & 5.173460 & 1.300199 & 4.023829 \\
\hline $\boldsymbol{\gamma}$ & 4.517090 & 2.488746 & 3.317974 \\
\hline ) & 0.677277 & -1.201256 & 0.386423 \\
\hline D & 0.874578 & 1.047395 & -0.820000 \\
\hline & -0.250138 & -0.080889 & -0.384118 \\
\hline & -1.258010 & -0.674423 & -1.920808 \\
\hline & -1.211363 & 0.670676 & 1.317492 \\
\hline & 5.780804 & -1.132754 & -3.678902 \\
\hline & 7.221689 & -2.045913 & -3.230411 \\
\hline
\end{tabular}




\begin{tabular}{|c|c|c|c|}
\hline $\mathrm{H}$ & 6.973705 & -1.198784 & -0.857391 \\
\hline $\mathrm{H}$ & 7.192868 & 0.125738 & -2.000365 \\
\hline $\mathrm{H}$ & 5.326022 & 0.564754 & -0.364492 \\
\hline $\mathrm{H}$ & 4.815710 & 0.628575 & -2.028836 \\
\hline & 5.981999 & -3.462666 & -1.710428 \\
\hline & 5.086748 & -3.440817 & -3.217864 \\
\hline $\mathrm{H}$ & 3.147163 & -4.220876 & -1.951187 \\
\hline $\mathrm{H}$ & 2.560970 & 3.966266 & 2.229853 \\
\hline $\mathrm{H}$ & 4.022383 & -0.931143 & 2.710446 \\
\hline $\mathrm{H}$ & 5.048954 & -0.841484 & 1.306737 \\
\hline $\mathrm{H}$ & 6.285306 & -0.489137 & 3.472459 \\
\hline $\mathrm{H}$ & 6.476004 & 0.864242 & 2.358641 \\
\hline $\mathrm{H}$ & 4.428068 & 0.773985 & 4.635843 \\
\hline $\mathrm{H}$ & 5.954224 & 1.655742 & 4.705998 \\
\hline $\mathrm{H}$ & 3.968598 & 3.109746 & 4.036160 \\
\hline $\mathrm{H}$ & 5.305845 & 3.133981 & 2.902346 \\
\hline C & 0.658482 & 3.695014 & 0.397125 \\
\hline C & -0.171418 & 4.156164 & 1.402915 \\
\hline C & -1.121610 & 5.172199 & 1.168986 \\
\hline C & -1.229259 & 5.736263 & -0.078190 \\
\hline C & -0.410554 & 5.290515 & -1.146501 \\
\hline C & -0.532048 & 5.846457 & -2.447174 \\
\hline C & 0.259757 & 5.407716 & -3.482411 \\
\hline C & 1.224136 & 4.398265 & -3.252804 \\
\hline C & 1.369488 & 3.841403 & -2.002970 \\
\hline $\mathrm{C}$ & 0.550246 & 4.251756 & -0.917568 \\
\hline $\mathrm{H}$ & -1.269943 & 6.628778 & -2.608222 \\
\hline $\mathrm{H}$ & 0.153002 & 5.837759 & -4.474320 \\
\hline $\mathrm{H}$ & 1.854928 & 4.061207 & -4.070407 \\
\hline $\mathrm{H}$ & 2.116853 & 3.074194 & -1.839839 \\
\hline $\mathrm{H}$ & -1.961296 & 6.514595 & -0.271921 \\
\hline $\mathrm{H}$ & -1.772097 & 5.494586 & 1.976016 \\
\hline $\mathrm{H}$ & -0.095672 & 3.709186 & 2.389078 \\
\hline C & 0.743828 & -3.849019 & -0.863288 \\
\hline C & 0.355018 & -4.363155 & -2.088770 \\
\hline C & -0.677027 & -5.318432 & -2.197561 \\
\hline C & -1.330466 & -5.755305 & -1.069533 \\
\hline C & -0.981302 & -5.249267 & 0.208597 \\
\hline C & -1.668160 & -5.6 & 1.376883 \\
\hline C & -1.336755 & -5.175551 & 2.613740 \\
\hline C & -0.270546 & -4.255191 & 2.737392 \\
\hline C & 0.426393 & -3.837017 & 1.627979 \\
\hline C & 0.077826 & -4.2 & 0.327337 \\
\hline $\mathrm{H}$ & 0.847181 & -4.004433 & -2.987197 \\
\hline $\mathrm{H}$ & -0.953630 & -5.698488 & -3.176841 \\
\hline $\mathrm{H}$ & -2.129264 & -6.489610 & -1.139141 \\
\hline $\mathrm{H}$ & -2.474436 & -6.396443 & 1.269270 \\
\hline $\mathrm{H}$ & -1.886293 & -5.487518 & 3.496573 \\
\hline $\mathrm{H}$ & -0.002356 & -3.873513 & 3.717855 \\
\hline $\mathrm{H}$ & 1.248598 & -3.142748 & 1.744151 \\
\hline C & -3.946072 & -3.189298 & 0.050719 \\
\hline C & -4.287658 & -1.952579 & 0.443877 \\
\hline C & -3.807026 & -1.375070 & 1.733123 \\
\hline C & -3.879665 & 0.150956 & 1.861081 \\
\hline C & -2.995692 & 0.786602 & 0.755150 \\
\hline 0 & -3.355178 & -2.082957 & 2.617428 \\
\hline C & -3.562099 & 0.585059 & 3.294158 \\
\hline $\mathrm{H}$ & -3.428089 & 1.667409 & 3.369536 \\
\hline
\end{tabular}




\begin{tabular}{|c|c|c|c|}
\hline $\mathrm{H}$ & -4.371629 & 0.287778 & 3.967794 \\
\hline $\mathrm{H}$ & -2.652687 & 0.093735 & 3.646326 \\
\hline $\mathrm{O}$ & -5.026394 & -1.071264 & -0.309016 \\
\hline $\mathrm{C}$ & -5.662010 & -1.640250 & -1.460978 \\
\hline C & -4.718137 & -2.558323 & -2.228334 \\
\hline $\mathrm{H}$ & -6.549988 & -2.196250 & -1.125808 \\
\hline $\mathrm{H}$ & -5.987463 & -0.785856 & -2.058722 \\
\hline $\mathrm{C}$ & -4.263516 & -3.709036 & -1.320322 \\
\hline $\mathrm{H}$ & -3.846640 & -1.976280 & -2.546234 \\
\hline $\mathrm{H}$ & -5.037313 & -4.490354 & -1.264146 \\
\hline $\mathrm{H}$ & -3.372072 & -4.191980 & -1.735157 \\
\hline $\mathrm{C}$ & -3.399646 & 2.173652 & 0.294199 \\
\hline C & -4.280192 & 2.992774 & 1.009292 \\
\hline C & -2.952145 & 2.612743 & -0.960741 \\
\hline C & -4.718510 & 4.207113 & 0.476295 \\
\hline $\mathrm{H}$ & -4.657019 & 2.681332 & 1.976318 \\
\hline C & -3.395346 & 3.816654 & -1.497107 \\
\hline $\mathrm{H}$ & -2.267040 & 1.993681 & -1.530597 \\
\hline C & -4.286761 & 4.618639 & -0.782001 \\
\hline $\mathrm{H}$ & -5.409354 & 4.822715 & 1.046160 \\
\hline $\mathrm{H}$ & -3.034522 & 4.131203 & -2.471411 \\
\hline $\mathrm{H}$ & -4.637001 & 5.557905 & -1.201522 \\
\hline $\mathrm{H}$ & -5.218979 & -2.932185 & -3.128409 \\
\hline $\mathrm{H}$ & -3.035896 & 0.146367 & -0.126466 \\
\hline $\mathrm{H}$ & -3.362970 & -3.784334 & 0.742917 \\
\hline $\mathrm{H}$ & -4.904776 & 0.433581 & 1.594827 \\
\hline \multicolumn{4}{|c|}{ imaginary frequencies } \\
\hline \multicolumn{4}{|c|}{$E_{\text {chloroform }}=-3563.784491$} \\
\hline & roform $=-356$ & & \\
\hline
\end{tabular}

\section{TS8}

\begin{tabular}{|c|c|c|c|}
\hline$C$ & 4.088472 & 4.886768 & -2.624148 \\
\hline & 3.475084 & 5.316884 & -1.290854 \\
\hline & 2.302086 & 4.404176 & -0.926448 \\
\hline & 2.627739 & 2.924314 & -1.045988 \\
\hline & 3.696352 & 2.491667 & -1.848082 \\
\hline & 4.654201 & 3.469870 & -2.504625 \\
\hline & 1.801681 & 1.961065 & -0.424835 \\
\hline & 1.975237 & 0.607652 & -0.732884 \\
\hline & 3.059611 & 0.152047 & -1.490443 \\
\hline & 3.915573 & 1.121622 & -2.015745 \\
\hline & 0.761356 & 2.352291 & 0.569900 \\
\hline & 1.108969 & 2.980421 & 1.786573 \\
\hline & 0.081506 & 3.396091 & 2.649794 \\
\hline & -1.249523 & 3.154025 & 2.303579 \\
\hline & -1.623645 & 2.454091 & 1.150957 \\
\hline & -0.576805 & 2.046666 & 0.312124 \\
\hline & 2.565360 & 3.099798 & 2.205461 \\
\hline & 2.798927 & 4.120176 & 3.321995 \\
\hline & 1.798430 & 3.907992 & 4.458789 \\
\hline & 0.374677 & 4.132234 & 3.944897 \\
\hline & 1.044469 & -0.312157 & -0.229304 \\
\hline & -0.858356 & 1.303425 & -0.838726 \\
\hline & -0.480015 & -0.294131 & -0.892033 \\
\hline & -0.675069 & -0.960701 & -2.695472 \\
\hline & -1.574161 & -1.162110 & 0.662526 \\
\hline & 3.313825 & 4.912495 & -3.402705 \\
\hline & 4.878925 & 5.578725 & -2.936591 \\
\hline
\end{tabular}




\begin{tabular}{|c|c|c|c|}
\hline & 4.241780 & 5.267009 & -0.504218 \\
\hline $\mathrm{H}$ & 3.131416 & 6.356729 & -1.333756 \\
\hline & 1.928936 & 4.643432 & 0.071356 \\
\hline & 1.462311 & 4.612302 & -1.606771 \\
\hline & 5.579063 & 3.505802 & -1.909101 \\
\hline & 4.946461 & 3.087208 & -3.489691 \\
\hline & 4.769851 & 0.788403 & -2.599625 \\
\hline & -2.036871 & 3.503176 & 2.965915 \\
\hline & 2.889100 & 2.106699 & 2.553420 \\
\hline & 3.203974 & 3.325553 & 1.349458 \\
\hline & 3.829919 & 4.036692 & 3.684745 \\
\hline & 2.683708 & 5.137859 & 2.922274 \\
\hline $\mathrm{H}$ & 1.897589 & 2.883125 & 4.842165 \\
\hline & 2.004179 & 4.584349 & 5.296347 \\
\hline $\mathrm{H}$ & -0.360376 & 3.840059 & 4.704109 \\
\hline & 0.223824 & 5.209429 & 3.777451 \\
\hline C & -3.057782 & 2.141665 & 0.923693 \\
\hline C & -3.768803 & 1.583501 & 1.973403 \\
\hline C & -5.146872 & 1.303822 & 1.869635 \\
\hline C & -5.811608 & 1.561767 & 0.695208 \\
\hline C & -5.127971 & 2.115573 & -0.415783 \\
\hline C & -5.804770 & 2.370348 & -1.637292 \\
\hline C & -5.153174 & 2.951020 & -2.698831 \\
\hline C & -3.793560 & 3.320275 & -2.572941 \\
\hline C & -3.106528 & 3.076689 & -1.406344 \\
\hline C & -3.735599 & 2.441179 & -0.302919 \\
\hline $\mathrm{H}$ & -6.853959 & 2.096502 & -1.713619 \\
\hline $\mathrm{H}$ & -5.679963 & 3.136872 & -3.630482 \\
\hline $\mathrm{H}$ & -3.286811 & 3.798653 & -3.406128 \\
\hline $\mathrm{H}$ & -2.068351 & 3.372624 & -1.325961 \\
\hline $\mathrm{H}$ & -6.870939 & 1.341562 & 0.596050 \\
\hline $\mathrm{H}$ & -5.670228 & 0.870303 & 2.717067 \\
\hline $\mathrm{H}$ & -3.243159 & 1.337497 & 2.890770 \\
\hline C & 3.380631 & -1.291033 & -1.680233 \\
\hline C & 2.735373 & -2.050593 & -2.633796 \\
\hline C & 3.083143 & -3.404929 & -2.846210 \\
\hline C & 4.078225 & -3.992771 & -2.101879 \\
\hline C & 4.766279 & -3.252023 & -1.105452 \\
\hline C & 5.778001 & -3.844282 & -0.304279 \\
\hline $\mathrm{C}$ & 6.430696 & -3.117926 & 0.665078 \\
\hline C & 6.096551 & -1.758901 & 0.874927 \\
\hline C & 5.117443 & -1.1556 & 0.118412 \\
\hline C & 4.421815 & -1.877098 & -0.889583 \\
\hline $\mathrm{H}$ & 1.945723 & -1.599970 & -3.222196 \\
\hline $\mathrm{H}$ & 2.553365 & -3.975795 & -3.603046 \\
\hline $\mathrm{H}$ & 4.347695 & -5.034217 & -2.258333 \\
\hline $\mathrm{H}$ & 6.027513 & -4.888931 & -0.472901 \\
\hline $\mathrm{H}$ & 7.202405 & -3.584272 & 1.270807 \\
\hline $\mathrm{H}$ & 6.612810 & -1.191104 & 1.643858 \\
\hline $\mathrm{H}$ & 4.856925 & -0.117750 & 0.293120 \\
\hline $\mathrm{C}$ & 0.532515 & -3.432774 & 3.212596 \\
\hline C & -0.135685 & -3.622490 & 2.062750 \\
\hline C & -1.624508 & -3.703024 & 2.020695 \\
\hline C & -2.267055 & -3.950284 & 0.650405 \\
\hline C & -2.343865 & -2.634978 & -0.227300 \\
\hline 0 & -2.299872 & -3.580021 & 3.031059 \\
\hline C & -3.603100 & -4.674986 & 0.828698 \\
\hline $\mathrm{H}$ & -4.084916 & -4.850346 & -0.136427 \\
\hline
\end{tabular}




$\begin{array}{lrrr}\mathrm{H} & -3.426072 & -5.642175 & 1.310883 \\ \mathrm{H} & -4.286680 & -4.108202 & 1.461963 \\ \mathrm{O} & 0.440985 & -3.716120 & 0.827836 \\ \mathrm{C} & 1.876415 & -3.781391 & 0.808079 \\ \mathrm{C} & 2.506530 & -2.843954 & 1.828428 \\ \mathrm{H} & 2.174076 & -4.821387 & 1.006543 \\ \mathrm{H} & 2.154712 & -3.524310 & -0.211695 \\ \mathrm{C} & 2.014682 & -3.199265 & 3.238012 \\ \mathrm{H} & 2.217760 & -1.818277 & 1.577137 \\ \mathrm{H} & 2.541202 & -4.088085 & 3.618624 \\ \mathrm{H} & 2.249863 & -2.390024 & 3.941768 \\ \mathrm{C} & -3.720327 & -2.271496 & -0.722622 \\ \mathrm{C} & -4.018622 & -2.433342 & -2.079747 \\ \mathrm{C} & -4.720176 & -1.807161 & 0.139926 \\ \mathrm{C} & -5.294461 & -2.145894 & -2.566576 \\ \mathrm{H} & -3.241734 & -2.771270 & -2.758246 \\ \mathrm{C} & -5.997172 & -1.536861 & -0.341551 \\ \mathrm{H} & -4.491835 & -1.646363 & 1.189130 \\ \mathrm{C} & -6.289341 & -1.702502 & -1.696978 \\ \mathrm{H} & -5.506132 & -2.264945 & -3.625429 \\ \mathrm{H} & -6.763585 & -1.186444 & 0.342111 \\ \mathrm{H} & -7.284796 & -1.481088 & -2.071862 \\ \mathrm{H} & 3.595118 & -2.911100 & 1.755021 \\ \mathrm{H} & -1.695382 & -2.788052 & -1.088074 \\ \mathrm{H} & -0.055329 & -3.389176 & 4.122686 \\ \mathrm{H} & -1.582460 & -4.604427 & 0.104918 \\ \mathrm{I} & \text { imaginary frequency } & \\ \mathrm{E}_{\text {chloroform }}=-3563.775460 & \\ \mathrm{G}_{\mathrm{chloroform}}=-3562.934917 & \\ & & & \end{array}$

14

$\begin{array}{lrrr}\text { C } & 6.905211 & -2.002247 & -2.381055 \\ \text { C } & 7.095352 & -1.049947 & -1.199443 \\ \text { C } & 5.787335 & -0.317040 & -0.894661 \\ \text { C } & 4.587229 & -1.243270 & -0.785147 \\ \text { C } & 4.603304 & -2.504725 & -1.404850 \\ \text { C } & 5.866462 & -3.070217 & -2.030228 \\ \text { C } & 3.399977 & -0.800676 & -0.159754 \\ \text { C } & 2.241262 & -1.573094 & -0.287874 \\ \text { C } & 2.228819 & -2.831296 & -0.899554 \\ \text { C } & 3.440440 & -3.281496 & -1.426829 \\ \text { C } & 3.331481 & 0.473737 & 0.615681 \\ \text { C } & 4.111213 & 0.678889 & 1.776145 \\ \text { C } & 4.027970 & 1.915061 & 2.441471 \\ \text { C } & 3.122040 & 2.880606 & 1.996626 \\ \text { C } & 2.260846 & 2.669622 & 0.915400 \\ \text { C } & 2.409468 & 1.453231 & 0.232102 \\ \text { C } & 4.925435 & -0.458016 & 2.372947 \\ \text { C } & 6.015199 & 0.012414 & 3.338673 \\ \text { C } & 5.436644 & 0.996167 & 4.356569 \\ \text { C } & 4.907239 & 2.238428 & 3.636557 \\ \text { O } & 1.031473 & -1.041690 & 0.177543 \\ \text { O } & 1.598465 & 1.193419 & -0.873853 \\ \text { P } & 0.356272 & 0.124186 & -0.785510 \\ \text { S } & -0.234680 & -0.382415 & -2.550317 \\ \text { S } & -1.008356 & 0.954420 & 0.567074 \\ \text { H } & 6.567015 & -1.428826 & -3.254977 \\ \text { H } & 7.851708 & -2.480155 & -2.659029\end{array}$




\begin{tabular}{|c|c|c|c|}
\hline $\mathrm{H}$ & 7.414083 & -1.622736 & -0.316595 \\
\hline $\mathrm{H}$ & 7.886189 & -0.319984 & -1.407318 \\
\hline $\mathrm{H}$ & 5.893267 & 0.297876 & 0.001049 \\
\hline $\mathrm{H}$ & 5.578642 & 0.393216 & -1.709178 \\
\hline & 6.316334 & -3.782511 & -1.321943 \\
\hline & 5.602415 & -3.656761 & -2.918262 \\
\hline $\mathrm{H}$ & 3.465683 & -4.261239 & -1.896983 \\
\hline $\mathrm{H}$ & 3.051019 & 3.823947 & 2.531739 \\
\hline & 4.225185 & -1.110754 & 2.916984 \\
\hline $\mathrm{H}$ & 5.356800 & -1.088849 & 1.594284 \\
\hline $\mathrm{H}$ & 6.460355 & -0.854804 & 3.840039 \\
\hline $\mathrm{H}$ & 6.821683 & 0.503489 & 2.775420 \\
\hline $\mathrm{H}$ & 4.619224 & 0.508687 & 4.905638 \\
\hline $\mathrm{H}$ & 6.190819 & 1.286619 & 5.097139 \\
\hline $\mathrm{H}$ & 4.355737 & 2.882095 & 4.332139 \\
\hline $\mathrm{H}$ & 5.763164 & 2.835917 & 3.287612 \\
\hline C & 1.141955 & 3.607985 & 0.642703 \\
\hline C & 0.319341 & 3.940237 & 1.706551 \\
\hline C & -0.861273 & 4.688758 & 1.522686 \\
\hline C & -1.224789 & 5.100310 & 0.263578 \\
\hline C & -0.394013 & 4.824744 & -0.851803 \\
\hline C & -0.745671 & 5.265115 & -2.155364 \\
\hline C & 0.079516 & 5.02 & -3.228477 \\
\hline C & 1.305559 & 4.345982 & -3.038632 \\
\hline C & 1.667335 & 3.894192 & -1.791025 \\
\hline $\mathrm{C}$ & 0.824719 & 4.094214 & -0.666072 \\
\hline $\mathrm{H}$ & -1.683906 & 5.798280 & -2.283676 \\
\hline $\mathrm{H}$ & -0.201095 & 5.366169 & -4.221628 \\
\hline $\mathrm{H}$ & 1.962723 & 4.174782 & -3.886306 \\
\hline $\mathrm{H}$ & 2.608714 & 3.374489 & -1.656809 \\
\hline $\mathrm{H}$ & -2.158183 & 5.6294 & 0.102423 \\
\hline $\mathrm{H}$ & -1.502273 & 4.897877 & 2.373906 \\
\hline $\mathrm{H}$ & 0.553238 & 3.549125 & 2.692104 \\
\hline C & 0.983927 & -3.628022 & -1.049740 \\
\hline C & 0.568686 & -3.971980 & -2.322501 \\
\hline C & -0.616339 & -4.708704 & -2.536950 \\
\hline C & -1.385098 & -5.104821 & -1.468838 \\
\hline C & -0.993452 & -4.790169 & -0.142268 \\
\hline C & -1.774645 & -5.194375 & 0.972653 \\
\hline C & -1.391803 & -4.883454 & 2.256045 \\
\hline C & -0.195270 & -4.162271 & 2.479494 \\
\hline C & 0.586627 & -3.760383 & 1.420846 \\
\hline C & 0.21 & -4.0 & 0.081086 \\
\hline $\mathrm{H}$ & 1.147971 & -3.628706 & -3.173485 \\
\hline $\mathrm{H}$ & -0.922425 & -4.943944 & -3.551988 \\
\hline $\mathrm{H}$ & -2.306048 & -5.661237 & -1.623645 \\
\hline $\mathrm{H}$ & -2.687567 & & 0.788262 \\
\hline $\mathrm{H}$ & -2.001130 & -5.194032 & 3.100141 \\
\hline $\mathrm{H}$ & 0.109309 & -3.927191 & 3.495390 \\
\hline $\mathrm{H}$ & 1.505945 & -3.216632 & 1.604893 \\
\hline C & -7.039208 & -1.520409 & -0.327092 \\
\hline C & -5.856112 & -1.287948 & 0.264955 \\
\hline C & -4.723801 & -0.642193 & -0.468013 \\
\hline C & -3.403665 & -0.484422 & 0.281633 \\
\hline C & -2.639939 & 0.710763 & -0.310210 \\
\hline 0 & -4.849449 & -0.303360 & -1.634307 \\
\hline C & -2.646107 & -1.820390 & 0.162781 \\
\hline $\mathrm{H}$ & 08210 & -1.785704 & 0.721811 \\
\hline
\end{tabular}




\begin{tabular}{|c|c|c|c|}
\hline $\mathrm{H}$ & -3.245403 & -2.635174 & 0.576310 \\
\hline & -2.415728 & -2.046380 & -0.881982 \\
\hline & -5.567971 & -1.608048 & 1.568353 \\
\hline C & -6.491955 & -2.518562 & 2.176383 \\
\hline C & -7.937394 & -2.102610 & 1.921951 \\
\hline $\mathrm{H}$ & -6.306760 & -3.523987 & 1.770679 \\
\hline $\mathrm{H}$ & -6.241602 & -2.518214 & 3.239637 \\
\hline C & -8.211551 & -2.098709 & 0.410800 \\
\hline $\mathrm{H}$ & -8.088261 & -1.097508 & 2.332883 \\
\hline $\mathrm{H}$ & -8.416457 & -3.119371 & 0.053948 \\
\hline $\mathrm{H}$ & -9.116758 & -1.519351 & 0.187004 \\
\hline C & -3.389063 & 2.020672 & -0.232313 \\
\hline C & -3.950683 & 2.473483 & 0.967183 \\
\hline C & -3.526589 & 2.804297 & -1.382218 \\
\hline C & -4.645157 & 3.681856 & 1.014022 \\
\hline $\mathrm{H}$ & -3.843651 & 1.881935 & 1.872333 \\
\hline C & -4.226831 & 4.008362 & -1.339792 \\
\hline $\mathrm{H}$ & -3.086819 & 2.460922 & -2.313417 \\
\hline C & -4.790486 & 4.450811 & -0.141597 \\
\hline $\mathrm{H}$ & -5.076378 & 4.020099 & 1.952295 \\
\hline $\mathrm{H}$ & -4.333880 & 4.600036 & -2.244733 \\
\hline $\mathrm{H}$ & -5.339825 & 5.387777 & -0.108581 \\
\hline & -8.617265 & -2.780509 & 2.449805 \\
\hline $\mathrm{H}$ & -2.398345 & 0.496387 & -1.351905 \\
\hline $\mathrm{H}$ & -7.128002 & -1.242380 & -1.371392 \\
\hline $\mathrm{H}$ & -3.617884 & -0.308067 & 1.339278 \\
\hline \multicolumn{4}{|c|}{ imaginary frequencies } \\
\hline \multicolumn{4}{|c|}{$E_{\text {chloroform }}=-3563.789764$} \\
\hline \multicolumn{4}{|c|}{$\mathrm{G}_{\text {chloroform }}=-3562.950640$} \\
\hline
\end{tabular}

$\begin{array}{lrrr}\text { 13' } & & & \\ \text { C } & 2.692878 & 6.164966 & 1.814778 \\ \text { C } & 1.313092 & 5.784280 & 2.354065 \\ \text { C } & 0.705920 & 4.662460 & 1.509051 \\ \text { C } & 1.653346 & 3.496217 & 1.282021 \\ \text { C } & 3.044294 & 3.676078 & 1.384186 \\ \text { C } & 3.642073 & 4.968265 & 1.912960 \\ \text { C } & 1.153915 & 2.247541 & 0.848710 \\ \text { C } & 2.059574 & 1.279364 & 0.412149 \\ \text { C } & 3.447948 & 1.427547 & 0.502474 \\ \text { C } & 3.910281 & 2.638378 & 1.019998 \\ \text { C } & -0.305620 & 1.931495 & 0.803320 \\ \text { C } & -1.097534 & 1.905495 & 1.975032 \\ \text { C } & -2.471179 & 1.630806 & 1.862198 \\ \text { C } & -3.027534 & 1.384240 & 0.603893 \\ \text { C } & -2.261516 & 1.341403 & -0.561966 \\ \text { C } & -0.888843 & 1.585463 & -0.420690 \\ \text { C } & -0.447083 & 2.034190 & 3.343032 \\ \text { C } & -1.437776 & 2.324545 & 4.473456 \\ \text { C } & -2.642593 & 1.386308 & 4.388937 \\ \text { C } & -3.385429 & 1.621390 & 3.073457 \\ \text { O } & 1.549102 & 0.111045 & -0.149016 \\ \text { O } & -0.074196 & 1.479853 & -1.545470 \\ \text { P } & 0.944084 & 0.182716 & -1.689259 \\ \text { S } & 2.179818 & 0.480519 & -3.136645 \\ \text { S } & -0.199683 & -1.571629 & -1.725083 \\ \text { H } & 2.596198 & 6.476214 & 0.765643 \\ \text { H } & 3.109517 & 7.017156 & 2.364023 \\ & & & \end{array}$




\begin{tabular}{|c|c|c|c|}
\hline $\mathrm{H}$ & 1.408326 & 5.452596 & 3.398124 \\
\hline $\mathrm{H}$ & 0.640127 & 6.649505 & 2.355473 \\
\hline $\mathrm{H}$ & -0.237736 & 4.324324 & 1.941978 \\
\hline $\mathrm{H}$ & 0.441020 & 5.065454 & 0.519537 \\
\hline & 3.916033 & 4.819233 & 2.968515 \\
\hline & 4.580697 & 5.177956 & 1.386371 \\
\hline $\mathrm{H}$ & 4.983275 & 2.784510 & 1.115120 \\
\hline $\mathrm{H}$ & -4.095897 & 1.202642 & 0.521702 \\
\hline & 0.066117 & 1.084251 & 3.554382 \\
\hline $\mathrm{H}$ & 0.342611 & 2.787461 & 3.334956 \\
\hline $\mathrm{H}$ & -0.929237 & 2.225357 & 5.439374 \\
\hline $\mathrm{H}$ & -1.785990 & 3.364790 & 4.402936 \\
\hline $\mathrm{H}$ & -2.299028 & 0.343784 & 4.437594 \\
\hline $\mathrm{H}$ & -3.321091 & 1.540093 & 5.236352 \\
\hline $\mathrm{H}$ & -4.162915 & 0.862198 & 2.932323 \\
\hline $\mathrm{H}$ & -3.910757 & 2.586663 & 3.130432 \\
\hline C & -2.883272 & 1.007827 & -1.868370 \\
\hline C & -3.606222 & -0.165706 & -1.974667 \\
\hline C & -4.206082 & -0.552277 & -3.192291 \\
\hline C & -4.082693 & 0.243577 & -4.305781 \\
\hline C & -3.374134 & 1.470992 & -4.240288 \\
\hline C & -3.261023 & 2.316749 & -5.375378 \\
\hline C & -2.598405 & 3.518716 & -5.25 \\
\hline C & -2.026202 & 3.931343 & -4.072917 \\
\hline C & -2.112253 & 3.131937 & -2.956846 \\
\hline C & -2.770559 & 1.874454 & -3.003842 \\
\hline $\mathrm{H}$ & -3.714516 & 1.993492 & -6.309123 \\
\hline $\mathrm{H}$ & -2.516813 & 4.155790 & -6.175139 \\
\hline $\mathrm{H}$ & -1.512760 & 4.886783 & -4.013733 \\
\hline $\mathrm{H}$ & -1.673925 & 3.463447 & -2.023081 \\
\hline $\mathrm{H}$ & -4.527511 & -0.052269 & -5.252560 \\
\hline $\mathrm{H}$ & -4.749932 & -1.491343 & -3.246228 \\
\hline $\mathrm{H}$ & -3.678985 & -0.814396 & -1.110939 \\
\hline C & 4.366658 & 0.377172 & -0.004998 \\
\hline C & 5.270102 & 0.704496 & -0.998167 \\
\hline C & 6.102950 & -0.272980 & -1.585360 \\
\hline C & 6.014117 & -1.585437 & -1.187604 \\
\hline C & 5.114389 & -1.969359 & -0.159599 \\
\hline C & 5.00322 & -3.321337 & 0.261643 \\
\hline C & 4.137054 & -3.683550 & 1.265637 \\
\hline C & 3.363859 & -2.694930 & 1.917911 \\
\hline C & 3.448627 & -1.376132 & 1.533137 \\
\hline C & 4.29 & -0.9739 & 0.466096 \\
\hline $\mathrm{H}$ & 5.297365 & 1.724446 & -1.368349 \\
\hline $\mathrm{H}$ & 6.789734 & 0.016128 & -2.375332 \\
\hline $\mathrm{H}$ & 6.628837 & -2.349918 & -1.655891 \\
\hline $\mathrm{H}$ & 5.612690 & -4.070754 & -0.237102 \\
\hline $\mathrm{H}$ & 4.039681 & -4.724667 & 1.556911 \\
\hline $\mathrm{H}$ & 2.695380 & -2.981128 & 2.724897 \\
\hline $\mathrm{H}$ & 2.861925 & -0.623712 & 2.048166 \\
\hline C & -3.318088 & -2.281311 & 2.962064 \\
\hline C & -3.273534 & -2.266127 & 1.619255 \\
\hline C & -2.107918 & -2.491189 & 0.742237 \\
\hline C & -0.823003 & -2.150790 & 0.972645 \\
\hline C & 0.233942 & -2.417626 & -0.066468 \\
\hline C & -0.344685 & -1.469444 & 2.225130 \\
\hline $\mathrm{H}$ & 0.069576 & -2.185520 & 2.950368 \\
\hline $\mathrm{H}$ & 0.451135 & -0.763436 & 1.977528 \\
\hline
\end{tabular}




\begin{tabular}{|c|c|c|c|}
\hline $\mathrm{H}$ & -1.144441 & -0.914171 & 2.716690 \\
\hline $\mathrm{O}$ & -4.402317 & -2.104510 & 0.833335 \\
\hline C & -5.577259 & -1.618775 & 1.495126 \\
\hline C & -5.809124 & -2.336122 & 2.818072 \\
\hline $\mathrm{H}$ & -5.456074 & -0.539330 & 1.654963 \\
\hline $\mathrm{H}$ & -6.392509 & -1.776432 & 0.785558 \\
\hline C & -4.599497 & -2.122819 & 3.736897 \\
\hline $\mathrm{H}$ & -5.944080 & -3.405722 & 2.619417 \\
\hline $\mathrm{H}$ & -4.646927 & -1.127981 & 4.204776 \\
\hline $\mathrm{H}$ & -4.622461 & -2.843052 & 4.563945 \\
\hline C & 0.625194 & -3.857829 & -0.339144 \\
\hline C & -0.012778 & -4.939058 & 0.273168 \\
\hline C & 1.739800 & -4.103609 & -1.153540 \\
\hline C & 0.450292 & -6.241189 & 0.072912 \\
\hline $\mathrm{H}$ & -0.867693 & -4.762847 & 0.915062 \\
\hline C & 2.201328 & -5.400065 & -1.354751 \\
\hline $\mathrm{H}$ & 2.249119 & -3.268007 & -1.624501 \\
\hline C & 1.556039 & -6.477372 & -0.741914 \\
\hline $\mathrm{H}$ & -0.058513 & -7.071184 & 0.555973 \\
\hline $\mathrm{H}$ & 3.070163 & -5.568891 & -1.984738 \\
\hline $\mathrm{H}$ & 1.914443 & -7.491158 & -0.898541 \\
\hline $\mathrm{H}$ & -6.732036 & -1.966469 & 3.278309 \\
\hline $\mathrm{H}$ & 1.141604 & -1.909302 & 0.244533 \\
\hline $\mathrm{H}$ & -2.403476 & -2.473038 & 3.507155 \\
\hline $\mathrm{O}$ & -2.416379 & -3.093386 & -0.453175 \\
\hline $\mathrm{H}$ & -3.381116 & -3.049092 & -0.564846 \\
\hline 0 & \multicolumn{3}{|c|}{ imaginary frequencies } \\
\hline \multirow{2}{*}{\multicolumn{4}{|c|}{$\mathrm{E}_{\text {chloroform }}=-3563.774701$}} \\
\hline & roform $=-35$ & & \\
\hline
\end{tabular}

\section{TS7'}

This TS was located as the maximum in $G$ when the $C-S$ bond length of $13^{\prime}$ was stretched in $0.1 \AA$ increments.

\begin{tabular}{|c|c|c|c|}
\hline C & -7.298384 & -0.369985 & -2.016850 \\
\hline C & -7.030721 & -1.342762 & -0.867240 \\
\hline C & -5.525006 & -1.494906 & -0.641226 \\
\hline C & -4.789568 & -0.167933 & -0.548687 \\
\hline C & -5.340580 & 0.993055 & -1.118676 \\
\hline C & -6.758144 & 1.017685 & -1.663214 \\
\hline C & -3.492492 & -0.108779 & 0.00645 \\
\hline C & -2.743349 & 1.063508 & -0.137628 \\
\hline C & -3.264595 & 2.229978 & -0.712630 \\
\hline C & -4.582837 & 2.167301 & -1.16954 \\
\hline C & -2.868034 & -1.266378 & 0.71477 \\
\hline C & -3.419248 & -1.797534 & 1.90229 \\
\hline C & -2.806393 & -2.911751 & 2.5017 \\
\hline C & -1.635291 & -3.436175 & 1.9486 \\
\hline C & -1.01 & -2.876281 & 0.8282 \\
\hline C & -1.660698 & -1.783948 & 0.2296 \\
\hline C & -4.575232 & -1.093935 & 2.59543 \\
\hline C & -5.310423 & -1.975155 & 3.60765 \\
\hline C & -4.312216 & -2.661060 & 4.54144 \\
\hline C & -3.391965 & -3.579025 & 3.73370 \\
\hline $\mathrm{O}$ & -1.424039 & 1.056477 & 0.29405 \\
\hline $\mathrm{O}$ & -1.075842 & -1.177159 & -0.87299 \\
\hline$P$ & -0.304844 & 0.287137 & -0.66883 \\
\hline S & -0.062779 & 1.062018 & -2.44670 \\
\hline
\end{tabular}




\begin{tabular}{|c|c|c|c|}
\hline 5 & 1.248693 & 0.077402 & 0.597696 \\
\hline $\mathrm{H}$ & -6.802667 & -0.740326 & -2.924755 \\
\hline & -8.370054 & -0.308113 & -2.239892 \\
\hline & -7.506736 & -0.963595 & 0.048663 \\
\hline & -7.473654 & -2.324421 & -1.072569 \\
\hline & -5.331754 & -2.114371 & 0.236911 \\
\hline & -5.092451 & -2.051063 & -1.487044 \\
\hline & -7.416906 & 1.470492 & -0.906368 \\
\hline & -6.802929 & 1.681219 & -2.535206 \\
\hline & -5.020598 & 3.060684 & -1.608020 \\
\hline & -1.166279 & -4.295839 & 2.420909 \\
\hline & -4.158386 & -0.220110 & 3.119580 \\
\hline & -5.279274 & -0.681976 & 1.870614 \\
\hline & -6.024122 & -1.3 & 4.174861 \\
\hline & -5.895683 & -2.739775 & 3.076733 \\
\hline & -3.714317 & -1.895820 & 5.055526 \\
\hline & -4.829446 & -3.238078 & 5.317069 \\
\hline & -2.579201 & -3.960787 & 3387 \\
\hline & -3.966474 & -4.462445 & 3.415433 \\
\hline & 0.311823 & -3.369483 & 0.380085 \\
\hline & 1.340577 & -3.401056 & 1.304795 \\
\hline & 2.64 & -3.82 & 0.950913 \\
\hline & 2.912551 & -4.215343 & -0.337388 \\
\hline & 1.885263 & -4.231496 & -1.315034 \\
\hline & 2.137403 & -4.674196 & -2.641431 \\
\hline C & 1.136270 & -4.69 & -3.583866 \\
\hline C & -0.172004 & -4.287712 & -3.231838 \\
\hline C & -0.450967 & -3.861944 & -1.954067 \\
\hline C & 0.562376 & -3.806316 & -0.960224 \\
\hline $\mathrm{H}$ & 3.141514 & -5.007662 & -2.893684 \\
\hline $\mathrm{H}$ & 1.343373 & -5.033779 & -4.595315 \\
\hline $\mathrm{H}$ & -0.961769 & -4.312540 & -3.976901 \\
\hline $\mathrm{H}$ & -1.457716 & -3.559355 & -1.693884 \\
\hline $\mathrm{H}$ & 3.913567 & -4.526292 & -0.625591 \\
\hline $\mathrm{H}$ & 3.425449 & -3.821164 & 1.701930 \\
\hline $\mathrm{H}$ & 1.147000 & -3.047862 & 2.312511 \\
\hline C & -2.434229 & 3.443331 & -0.925303 \\
\hline C & -2.320704 & & -2.211184 \\
\hline C & -1.502419 & 5.050944 & -2.505156 \\
\hline C & -0.787767 & 5.665347 & -1.504920 \\
\hline C & -0.885244 & 5.208418 & -0.165376 \\
\hline C & -0.165 & & 0.882704 \\
\hline C & -0.277382 & 5.4 & 2.182365 \\
\hline C & -1.131443 & 4.321648 & 2.490883 \\
\hline C & -1.840037 & 3.685928 & 1.497962 \\
\hline C & -1.730080 & & 0.141210 \\
\hline $\mathrm{H}$ & -2.833067 & & -3.017421 \\
\hline $\mathrm{H}$ & -1.423971 & 5.399627 & -3.530873 \\
\hline $\mathrm{H}$ & -0.139170 & 6.510305 & -1.722970 \\
\hline $\mathrm{H}$ & 0.477000 & 6.683446 & 0.634376 \\
\hline $\mathrm{H}$ & 0.279697 & & 2.974700 \\
\hline $\mathrm{H}$ & -1.227503 & 3.988139 & 3.520257 \\
\hline $\mathrm{H}$ & -2.494379 & 2.859504 & 1.747572 \\
\hline C & 4.626352 & -0.183177 & 1.471004 \\
\hline C & 5.114869 & 1.067893 & 1.466515 \\
\hline $\mathrm{C}$ & 4.437472 & 2.269415 & 0.964668 \\
\hline C & 3.420435 & 2.375050 & 0.027557 \\
\hline C & 3.067811 & 1.368793 & -0.893855 \\
\hline
\end{tabular}




\begin{tabular}{|c|c|c|c|}
\hline & 2.572675 & 3.632142 & 0.052391 \\
\hline & 1.691015 & 3.507390 & -0.579683 \\
\hline & 2.232739 & 3.866310 & 1.064971 \\
\hline$t$ & 3.138111 & 4.498345 & -0.311259 \\
\hline & 6.307026 & 1.423752 & 2.075854 \\
\hline & 7.195571 & 0.332205 & 2.365854 \\
\hline & 6.459673 & -0.812451 & 3.050651 \\
\hline$t$ & 7.638861 & -0.004026 & 1.418747 \\
\hline & 7.980728 & 0.759975 & 2.992551 \\
\hline & 5.340296 & -1.321650 & 2.134309 \\
\hline & 6.036547 & -0.445298 & 3.992816 \\
\hline $\mathrm{H}$ & 5.741013 & -1.992241 & 1.359127 \\
\hline t & 4.616670 & -1.920898 & 2.699526 \\
\hline & 3.933037 & 0.359161 & -1.513261 \\
\hline 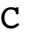 & 3.361826 & -0.786827 & -2.094772 \\
\hline C & 5.319324 & 0.565780 & -1.647272 \\
\hline & 4.160892 & -1.716716 & -2.751658 \\
\hline & 2.295203 & -0.952783 & -2.002898 \\
\hline$C$ & 6.113888 & -0.36 & -2.310124 \\
\hline$H$ & 5.762271 & 1.474342 & -1.254169 \\
\hline & 5.538199 & -1.512916 & -2.856621 \\
\hline $\mathrm{H}$ & 3.698413 & -2.597735 & -3.182858 \\
\hline t & 7.180290 & -0.182571 & -2. \\
\hline $\mathrm{H}$ & 6.159000 & -2.237876 & -3.376092 \\
\hline $\mathrm{H}$ & 7.168730 & -1.610247 & 3.296750 \\
\hline 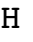 & 2.162960 & 1.567848 & -1.462371 \\
\hline F & 3.685874 & -0.38 & 0.978239 \\
\hline 0 & 4.783433 & 3.3 & 1.608725 \\
\hline $\mathrm{H}$ & 5.510081 & 3.174969 & 2.222947 \\
\hline \multicolumn{4}{|c|}{1 imaginary frequency } \\
\hline \multicolumn{4}{|c|}{$E_{\text {chloroform }}=-3563.743188$} \\
\hline \multicolumn{4}{|c|}{$\mathrm{G}_{\mathrm{chloroform}}=-3562.906201$} \\
\hline \multicolumn{4}{|c|}{$1 '$} \\
\hline C & -1.270164 & -1.912250 & 0.827362 \\
\hline$C$ & -1.209252 & -0.820515 & 0.046697 \\
\hline & -1.889748 & 0.452533 & 0.444230 \\
\hline & -1.534826 & 1.7328 & -0.254217 \\
\hline C & -0.271634 & 2.149859 & -0.463113 \\
\hline 0 & -2.764314 & 0.445370 & 1.303632 \\
\hline C & -2.727537 & 2.568686 & -0.641264 \\
\hline$\theta$ & -0.139045 & 3.084773 & -1.008603 \\
\hline $\mathrm{H}$ & -3.367578 & 2.03 & -1.358113 \\
\hline $\mathrm{n}$ & -2.419306 & 3.518637 & -1.086955 \\
\hline $\mathrm{H}$ & -3.342211 & 2.769966 & 0.242639 \\
\hline 0 & -0.530372 & -0.757911 & -1.137867 \\
\hline C & 0.469539 & -1.773245 & -1.294781 \\
\hline C & -0.098501 & -3.156747 & -0.992584 \\
\hline $\mathrm{H}$ & 0.812497 & -1.683616 & -2.327788 \\
\hline $\mathrm{H}$ & 1.304683 & -1.537238 & -0.622388 \\
\hline C & -0.597369 & -3.202661 & 0.459873 \\
\hline $\mathrm{H}$ & 0.664900 & -3.921652 & -1.174106 \\
\hline $\mathrm{H}$ & 0.242049 & -3.389211 & 1.147024 \\
\hline $\mathrm{H}$ & -1.289505 & -4.043203 & 0.601984 \\
\hline $\mathrm{H}$ & -1.829157 & -1.830986 & 1.752880 \\
\hline C & 0.971441 & 1.496765 & -0.028066 \\
\hline C & 2.091339 & 1.503794 & -0.876461 \\
\hline C & 1.088764 & 0.877180 & 1.226609 \\
\hline
\end{tabular}




$\begin{array}{lrrr}\mathrm{C} & 3.273671 & 0.869220 & -0.505652 \\ \mathrm{H} & 2.018655 & 1.994907 & -1.843408 \\ \mathrm{C} & 2.272826 & 0.242681 & 1.599460 \\ \mathrm{H} & 0.255161 & 0.914454 & 1.919731 \\ \mathrm{C} & 3.367469 & 0.230331 & 0.733708 \\ \mathrm{H} & 4.124547 & 0.874871 & -1.181306 \\ \mathrm{H} & 2.343490 & -0.230920 & 2.574708 \\ \mathrm{H} & 4.291734 & -0.259117 & 1.027495 \\ \mathrm{H} & -0.929532 & -3.350785 & -1.680720 \\ \mathrm{O} & \text { imaginary frequencies } & \\ \mathrm{E}_{\text {chloroform }}=-731.547429 & \\ \mathrm{G}_{\text {chloroform }}=-731.309505 & \\ \end{array}$

\section{TS9-up}

$\begin{array}{rrrr}\text { C } & 5.657518 & 4.090472 & 2.164726 \\ \mathrm{C} & 6.322934 & 2.827853 & 1.612991 \\ \mathrm{C} & 5.277963 & 1.727751 & 1.417720 \\ \mathrm{C} & 4.059940 & 2.186024 & 0.635084 \\ \mathrm{C} & 3.699452 & 3.546313 & 0.620588 \\ \mathrm{C} & 4.624269 & 4.620559 & 1.167054 \\ \mathrm{C} & 3.205808 & 1.241288 & 0.029739 \\ \mathrm{C} & 1.957510 & 1.663013 & -0.447855 \\ \mathrm{C} & 1.570945 & 3.008863 & -0.461028 \\ \mathrm{C} & 2.481458 & 3.934353 & 0.055775 \\ \mathrm{C} & 3.560298 & -0.204849 & -0.103517 \\ \mathrm{C} & 4.654170 & -0.643449 & -0.883800 \\ \mathrm{C} & 4.929276 & -2.020531 & -0.956932 \\ \mathrm{C} & 4.097064 & -2.932114 & -0.297600 \\ \mathrm{C} & 2.961153 & -2.531946 & 0.407687 \\ \mathrm{C} & 2.719812 & -1.154016 & 0.485599 \\ \mathrm{C} & 5.470565 & 0.356698 & -1.685831 \\ \mathrm{C} & 6.282985 & -0.296952 & -2.807015 \\ \mathrm{C} & 7.060962 & -1.492075 & -2.254857 \\ \mathrm{C} & 6.080788 & -2.568174 & -1.782923 \\ \mathrm{O} & 1.067278 & 0.706860 & -0.907099 \\ \mathrm{O} & 1.596219 & -0.698264 & 1.157206 \\ \mathrm{P} & 0.284556 & -0.227687 & 0.240223 \\ \mathrm{~S} & -0.738502 & 0.943267 & 1.500433 \\ \mathrm{~S} & -0.519982 & -1.711285 & -0.766730 \\ \mathrm{H} & 5.163795 & 3.848432 & 3.115953 \\ \mathrm{H} & 6.400978 & 4.867245 & 2.379192 \\ \mathrm{H} & 6.804550 & 3.058043 & 0.651631 \\ \mathrm{H} & 7.111024 & 2.474551 & 2.288329 \\ \mathrm{H} & 5.725030 & 0.840856 & 0.966610 \\ \mathrm{H} & 4.922813 & 1.399162 & 2.406534 \\ \mathrm{H} & 5.156443 & 5.084687 & 0.322518 \\ \mathrm{H} & 4.027973 & 5.421146 & 1.621405 \\ \mathrm{H} & 2.220645 & 4.989663 & 0.035396 \\ \mathrm{H} & 4.323170 & -3.993793 & -0.361174 \\ \mathrm{H} & 4.799909 & 1.122518 & -2.090362 \\ \mathrm{H} & 6.166767 & 0.894283 & -1.027788 \\ \mathrm{H} & 5.610581 & -0.638619 & -3.606088 \\ \mathrm{H} & 6.958057 & 0.443572 & -3.251653 \\ \mathrm{H} & 7.739251 & -1.908767 & -3.008512 \\ \mathrm{H} & 7.684184 & -1.158073 & -1.413304 \\ \mathrm{H} & 5.666678 & -3.080772 & -2.664016 \\ \mathrm{H} & 6.605286 & -3.341174 & -1.207011 \\ \mathrm{H} & -1.982724 & -0.571849 & 2.449091 \\ & & & \end{array}$




\begin{tabular}{|c|c|c|c|}
\hline & -3.483831 & 0.090252 & -0.762338 \\
\hline C & -3.539750 & 0.026050 & 0.623336 \\
\hline C & -3.257137 & -1.249013 & 1.249806 \\
\hline & -3.612272 & -2.342898 & 0.474185 \\
\hline & -4.485703 & -2.062984 & -0.613725 \\
\hline & -2.626763 & -1.342498 & 2.409470 \\
\hline C & -2.983149 & -3.692217 & 0.666424 \\
\hline & -2.089641 & -3.612682 & 1.287390 \\
\hline $\mathrm{H}$ & -2.689230 & -4.115807 & -0.300399 \\
\hline & -3.686338 & -4.392556 & 1.135438 \\
\hline 0 & -3.977792 & 1.017188 & 1.403717 \\
\hline & -4.000612 & 2.323153 & 0.767700 \\
\hline & -4.763236 & 2.239217 & -0.547183 \\
\hline & -2.962139 & 2.632870 & 0.622169 \\
\hline & -4.478371 & 2.978929 & 1.495061 \\
\hline & -4.051281 & 1.259641 & -1.495112 \\
\hline & -5.785765 & 1.900321 & -0.345627 \\
\hline $\mathrm{H}$ & -4.820410 & 3.234703 & -0.997753 \\
\hline & -3.235288 & 1.761661 & -2.031849 \\
\hline & -4.749173 & 0.893657 & -2.263238 \\
\hline & -2.742992 & -0.533821 & -1.252873 \\
\hline C & 2.0 & -3. & 0607 \\
\hline & 1.790048 & -3.403765 & 2.407876 \\
\hline & 0.900462 & -4.282303 & 3.062131 \\
\hline c & 0.248883 & -5.262381 & 2.351263 \\
\hline C & 0.4 & -5 & 158 \\
\hline C & -0.250112 & -6.368017 & 0.195868 \\
\hline C & -0.089769 & -6.456594 & -1.166990 \\
\hline C & 0.779162 & -5.560397 & -1.833579 \\
\hline C & 1.477970 & -4.6 & -1.127649 \\
\hline C & 1.351303 & -4.499257 & 0.283441 \\
\hline $\mathrm{H}$ & -0.924647 & -7.042183 & 0.718766 \\
\hline $\mathrm{H}$ & -0.633621 & -7.206685 & -1.734960 \\
\hline $\mathrm{H}$ & 0.8 & -5.6 & -2.9 \\
\hline $\mathrm{H}$ & 2.111310 & -3.902796 & -1.651041 \\
\hline $\mathrm{H}$ & -0.437719 & -5.945040 & 2.846234 \\
\hline $\mathrm{H}$ & 0.735063 & -4.176530 & 4.130482 \\
\hline $\mathrm{H}$ & & -2.6 & 77527 \\
\hline C & 0.275188 & 3.432573 & -1.055249 \\
\hline C & -0.002140 & 3.101216 & -2.369603 \\
\hline C & -1.180584 & 3.541421 & -3.009604 \\
\hline C & -2.0 & 4.324363 & -2.332383 \\
\hline C & -1.866219 & 4.664822 & -0.973118 \\
\hline C & -2.809197 & 5.437198 & -0.241861 \\
\hline C & -2.618131 & 5.709372 & 1.092744 \\
\hline C & -1.471949 & 5.211369 & 1.757534 \\
\hline C & -0.528072 & 4.4802 & 1.072779 \\
\hline C & -0.680308 & 4.199405 & -0.312409 \\
\hline $\mathrm{H}$ & 0.710228 & 2.496801 & -2.920447 \\
\hline $\mathrm{H}$ & -1.355304 & 3.272520 & -4.047713 \\
\hline $\mathrm{H}$ & -2.986161 & 4.688528 & -2.824900 \\
\hline $\mathrm{H}$ & -3.692608 & 5.803286 & -0.760255 \\
\hline $\mathrm{H}$ & -3.349033 & 6.297011 & 1.641497 \\
\hline $\mathrm{H}$ & -1.341532 & 5.400200 & 2.819138 \\
\hline $\mathrm{H}$ & 0.331764 & 4.076910 & 1.593188 \\
\hline C & -5.824744 & -1.472411 & -0.568168 \\
\hline C & -6.459629 & -1.115832 & 0.638138 \\
\hline C & -6.511399 & -1.256568 & -1.778400 \\
\hline
\end{tabular}




$\begin{array}{lrrr}\mathrm{C} & -7.719310 & -0.529050 & 0.623152 \\ \mathrm{H} & -5.962240 & -1.308015 & 1.582123 \\ \mathrm{C} & -7.771921 & -0.665914 & -1.788291 \\ \mathrm{H} & -6.039208 & -1.546799 & -2.713608 \\ \mathrm{C} & -8.378579 & -0.293258 & -0.587547 \\ \mathrm{H} & -8.196072 & -0.263157 & 1.562033 \\ \mathrm{H} & -8.282167 & -0.500115 & -2.732594 \\ \mathrm{H} & -9.364387 & 0.162059 & -0.592187 \\ \mathrm{H} & -4.295898 & -2.644688 & -1.518956 \\ 1 & \text { imaginary frequency } & \\ \mathrm{E}_{\text {chloroform }}=-3563.742768 & \\ \mathrm{G}_{\text {chloroform }}=-3562.906231 & \end{array}$

\section{TS9-down}

$\begin{array}{lrrr}\text { C } & -3.832740 & -5.712795 & 0.236578 \\ \mathrm{C} & -4.075826 & -4.921880 & -1.049776 \\ \mathrm{C} & -3.709832 & -3.449634 & -0.847584 \\ \mathrm{C} & -2.355874 & -3.246721 & -0.188935 \\ \mathrm{C} & -1.749912 & -4.281223 & 0.544271 \\ \mathrm{C} & -2.345411 & -5.676984 & 0.595443 \\ \mathrm{C} & -1.736169 & -1.979336 & -0.224577 \\ \mathrm{C} & -0.593325 & -1.753897 & 0.544550 \\ \mathrm{C} & 0.047665 & -2.777365 & 1.247127 \\ \mathrm{C} & -0.548863 & -4.037480 & 1.220213 \\ \mathrm{C} & -2.274389 & -0.847142 & -1.032052 \\ \mathrm{C} & -2.301199 & -0.879534 & -2.442515 \\ \mathrm{C} & -2.877311 & 0.198351 & -3.134556 \\ \mathrm{C} & -3.404002 & 1.276106 & -2.415361 \\ \mathrm{C} & -3.311504 & 1.371599 & -1.024968 \\ \mathrm{C} & -2.695419 & 0.303236 & -0.358465 \\ \mathrm{C} & -1.585146 & -1.993705 & -3.188111 \\ \mathrm{C} & -1.958380 & -2.079390 & -4.669987 \\ \mathrm{C} & -1.920876 & -0.692033 & -5.313349 \\ \mathrm{C} & -2.960775 & 0.214193 & -4.650070 \\ \mathrm{O} & -0.066085 & -0.469997 & 0.571528 \\ \mathrm{O} & -2.456690 & 0.379966 & 1.005021 \\ \mathrm{P} & -0.899027 & 0.762014 & 1.495667 \\ \mathrm{~S} & -0.758110 & 0.401611 & 3.406424 \\ \mathrm{~S} & -0.255730 & 2.429876 & 0.640914 \\ \mathrm{H} & -4.421057 & -5.268157 & 1.050916 \\ \mathrm{H} & -4.166709 & -6.751544 & 0.130142 \\ \mathrm{H} & -3.464074 & -5.348107 & -1.858048 \\ \mathrm{H} & -5.121832 & -5.000670 & -1.368118 \\ \mathrm{H} & -3.764052 & -2.910461 & -1.796305 \\ \mathrm{H} & -4.466263 & -2.976268 & -0.203375 \\ \mathrm{H} & -1.795907 & -6.321556 & -0.107737 \\ \mathrm{H} & -2.179004 & -6.107404 & 1.590260 \\ \mathrm{H} & -0.065235 & -4.851698 & 1.754386 \\ \mathrm{H} & -3.878422 & 2.091661 & -2.955695 \\ \mathrm{H} & -0.504779 & -1.799988 & -3.099965 \\ \mathrm{H} & -1.747553 & -2.958412 & -2.700991 \\ \mathrm{H} & -1.277221 & -2.769316 & -5.182153 \\ \mathrm{H} & -2.970266 & -2.496448 & -4.773856 \\ \mathrm{H} & -0.917607 & -0.260601 & -5.184282 \\ \mathrm{H} & -2.107731 & -0.752654 & -6.392061 \\ \mathrm{H} & -2.860416 & 1.245144 & -5.010615 \\ \mathrm{H} & -3.964296 & -0.116505 & -4.958622 \\ \mathrm{H} & 0.743672 & -0.120950 & -0.774410\end{array}$




\begin{tabular}{|c|c|c|c|}
\hline & -3.803415 & 2.579110 & -0.316011 \\
\hline & -3.321760 & 3.817227 & -0.697650 \\
\hline & -3.751289 & 5.002574 & -0.063710 \\
\hline & -4.668376 & 4.944647 & 0.958220 \\
\hline & -5.204631 & 3.699804 & 1.376631 \\
\hline & -6.166185 & 3.624450 & 2.418824 \\
\hline & -6.699240 & 2.417360 & 2.804428 \\
\hline & -6.295599 & 1.228305 & 2.152974 \\
\hline & -5.362919 & 1.266767 & 1.142628 \\
\hline & -4.778096 & 2.493410 & 0.729898 \\
\hline & -6.474550 & 4.546199 & 2.906360 \\
\hline & -7.432128 & 2.373040 & 3.605289 \\
\hline & -6.724106 & 0.277031 & 2.455793 \\
\hline & -5.064898 & 0.349169 & 0.649582 \\
\hline & -4.996801 & 5.850272 & 1.462310 \\
\hline & -3.338645 & 5.957072 & -0.378252 \\
\hline & -2.564651 & 3.866049 & -1.473400 \\
\hline & 1.356326 & -2.568971 & 1.926588 \\
\hline & 1.426914 & -2.413737 & 3.294999 \\
\hline & 2.673575 & -2.278049 & 3.949575 \\
\hline & 3.845860 & -2.310219 & 3.230171 \\
\hline & 3.820783 & -2.470007 & 1.819585 \\
\hline & 5.014377 & -2.532061 & 1.053522 \\
\hline & 4.972690 & -2.697608 & -0.312418 \\
\hline & 3.726011 & -2.789468 & -0.976409 \\
\hline & 2.551118 & -2.741073 & -0.260826 \\
\hline & 2.558812 & -2.590745 & 1.151643 \\
\hline & 0.507772 & -2.372077 & 3.867429 \\
\hline & 2.695733 & -2.150209 & 5.027983 \\
\hline 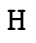 & 4.807412 & -2.219274 & 3.729982 \\
\hline 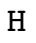 & 5.967454 & -2.460831 & 1.572898 \\
\hline & 5.893941 & -2.759993 & -0.885548 \\
\hline & 3.697704 & -2.910786 & -2.055680 \\
\hline & 1.599754 & -2.823357 & -0.773645 \\
\hline 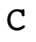 & 2.937459 & 2.804686 & -0.047191 \\
\hline C & 2.563917 & 1.467231 & -0.141555 \\
\hline 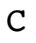 & 2.188345 & 0.951299 & -1.442386 \\
\hline$C$ & 2.819650 & 1.601118 & -2.492620 \\
\hline C & 3.955700 & 2.395167 & -2.162460 \\
\hline 0 & 1.288536 & 0.006010 & -1.613492 \\
\hline C & 2.254777 & 1.574692 & -3.885588 \\
\hline $\mathrm{H}$ & 2.390747 & 2.547181 & -4.373696 \\
\hline $\mathrm{H}$ & 2.767790 & 0.829148 & -4.506412 \\
\hline $\mathrm{H}$ & 1.190686 & 1.324228 & -3.867408 \\
\hline 0 & 2.680683 & 0.590782 & 0.841912 \\
\hline C & 2.884997 & 1.141358 & 2.175511 \\
\hline C & 4.017239 & 2.156459 & 2.124830 \\
\hline $\mathrm{H}$ & 1.933847 & 1.577988 & 2.493174 \\
\hline $\mathrm{H}$ & 3.111053 & 0.271458 & 2.788598 \\
\hline C & 3.656642 & 3.302939 & 1.162915 \\
\hline $\mathrm{H}$ & 4.929648 & 1.653672 & 1.786191 \\
\hline $\mathrm{H}$ & 3.021796 & 4.049018 & 1.658938 \\
\hline $\mathrm{H}$ & 4.566322 & 3.831740 & 0.840467 \\
\hline $\mathrm{H}$ & 2.446537 & 3.521300 & -0.688199 \\
\hline $\mathrm{H}$ & 4.206020 & 2.546773 & 3.130050 \\
\hline C & 5.208377 & 2.003731 & -1.514485 \\
\hline C & 5.465705 & 0.699478 & -1.046975 \\
\hline C & 6.205000 & 2.986353 & -1.353973 \\
\hline
\end{tabular}




\begin{tabular}{lrrr}
$\mathrm{C}$ & 6.670104 & 0.408940 & -0.418912 \\
$\mathrm{H}$ & 4.725987 & -0.083522 & -1.169554 \\
$\mathrm{C}$ & 7.409310 & 2.688307 & -0.722255 \\
$\mathrm{H}$ & 6.021459 & 3.992207 & -1.723829 \\
$\mathrm{C}$ & 7.644745 & 1.397843 & -0.247432 \\
$\mathrm{H}$ & 6.845684 & -0.598992 & -0.060153 \\
$\mathrm{H}$ & 8.162327 & 3.461827 & -0.603825 \\
$\mathrm{H}$ & 8.583320 & 1.159176 & 0.243775 \\
$\mathrm{H}$ & 4.061044 & 3.306246 & -2.758617 \\
$\mathrm{l}$ imaginary frequency & \\
$\mathrm{E}_{\text {chloroform }}=-3563.741331$ & \\
$\mathrm{G}_{\text {chloroform }}=-3562.905225$ & \\
\multicolumn{2}{l}{}
\end{tabular}

\section{Protonated divinyl ketone $1 \mathrm{H}^{+}$}

$\begin{array}{lrrr}\mathrm{C} & 1.845933 & -0.967630 & 0.690388 \\ \mathrm{C} & 2.083510 & 0.144608 & -0.045803 \\ \mathrm{C} & 1.186513 & 1.290690 & -0.142128 \\ \mathrm{C} & -0.225125 & 1.292227 & 0.014772 \\ \mathrm{C} & -0.897618 & 0.109848 & -0.249833 \\ \mathrm{O} & 1.780429 & 2.446125 & -0.360576 \\ \mathrm{C} & -0.897959 & 2.613831 & 0.306422 \\ \mathrm{H} & -0.303351 & -0.692144 & -0.678074 \\ \mathrm{H} & -1.063167 & 2.746043 & 1.382381 \\ \mathrm{H} & -1.863703 & 2.678073 & -0.198845 \\ \mathrm{H} & -0.276519 & 3.444158 & -0.031247 \\ \mathrm{O} & 3.278350 & 0.433722 & -0.640051 \\ \mathrm{C} & 4.243883 & -0.648425 & -0.723121 \\ \mathrm{C} & 4.291794 & -1.445838 & 0.570758 \\ \mathrm{H} & 5.190299 & -0.157699 & -0.951014 \\ \mathrm{H} & 3.953050 & -1.277003 & -1.572606 \\ \mathrm{C} & 2.900741 & -2.011137 & 0.896310 \\ \mathrm{H} & 5.025331 & -2.251068 & 0.469633 \\ \mathrm{H} & 2.856297 & -2.374873 & 1.928713 \\ \mathrm{H} & 2.678332 & -2.882765 & 0.262479 \\ \mathrm{H} & 0.897506 & -1.062209 & 1.203601 \\ \mathrm{C} & -2.294850 & -0.201074 & -0.133771 \\ \mathrm{C} & -2.763236 & -1.333356 & -0.846549 \\ \mathrm{C} & -3.214598 & 0.521069 & 0.666313 \\ \mathrm{C} & -4.098747 & -1.702073 & -0.800870 \\ \mathrm{H} & -2.062925 & -1.906089 & -1.448272 \\ \mathrm{C} & -4.545394 & 0.134559 & 0.723859 \\ \mathrm{H} & -2.879016 & 1.354399 & 1.268600 \\ \mathrm{C} & -4.993125 & -0.966190 & -0.016258 \\ \mathrm{H} & -4.446205 & -2.561745 & -1.364158 \\ \mathrm{H} & -5.239916 & 0.685820 & 1.349204 \\ \mathrm{H} & -6.038038 & -1.257196 & 0.028786 \\ \mathrm{H} & 4.630000 & -0.795210 & 1.384565 \\ \mathrm{H} & 2.741458 & 2.284074 & -0.491656 \\ \mathrm{O} & \mathrm{imaginary} \mathrm{frequencies} & \\ \mathrm{E} \text { chloroform } & =-731.960333 & \\ \mathrm{G} \text { chloroform } & =-731.708303 & \\ & & & \end{array}$

TS for electrocyclization of protonated divinyl ketone $1 \mathrm{H}^{+}$in the absence of any anion

$\begin{array}{lrrr}\mathrm{H} & 3.205449 & 2.407116 & 0.166283 \\ \mathrm{C} & 1.047974 & -0.726590 & 0.618280 \\ \mathrm{C} & 1.991955 & 0.238754 & 0.223283\end{array}$




$\begin{array}{lrrr}\mathrm{C} & 1.511559 & 1.555370 & -0.050149 \\ \mathrm{C} & 0.151760 & 1.605959 & -0.303496 \\ \mathrm{C} & -0.440996 & 0.313104 & -0.579112 \\ \mathrm{O} & 2.295220 & 2.639563 & -0.089637 \\ \mathrm{C} & -0.599232 & 2.905220 & -0.338807 \\ \mathrm{H} & -0.009355 & -0.239934 & -1.409060 \\ \mathrm{H} & -0.065270 & 3.607968 & -0.989061 \\ \mathrm{H} & -0.642777 & 3.370573 & 0.653165 \\ \mathrm{H} & -1.614660 & 2.776681 & -0.714380 \\ \mathrm{O} & 3.270094 & -0.012186 & -0.044938 \\ \mathrm{C} & 3.731359 & -1.374182 & 0.227830 \\ \mathrm{C} & 2.706334 & -2.376850 & -0.285062 \\ \mathrm{H} & 3.886613 & -1.460869 & 1.308964 \\ \mathrm{H} & 4.690363 & -1.439459 & -0.284333 \\ \mathrm{C} & 1.375194 & -2.186711 & 0.460411 \\ \mathrm{H} & 2.576684 & -2.227161 & -1.363341 \\ \mathrm{H} & 1.422244 & -2.646842 & 1.455896 \\ \mathrm{H} & 0.552965 & -2.694643 & -0.060670 \\ \mathrm{H} & 0.344638 & -0.459388 & 1.395629 \\ \mathrm{C} & -1.813470 & -0.042491 & -0.272484 \\ \mathrm{C} & -2.507071 & 0.537760 & 0.809975 \\ \mathrm{C} & -2.455929 & -1.027909 & -1.048943 \\ \mathrm{C} & -3.813549 & 0.158950 & 1.087886 \\ \mathrm{H} & -2.013037 & 1.275380 & 1.435377 \\ \mathrm{C} & -3.765768 & -1.401243 & -0.770894 \\ \mathrm{H} & -1.924290 & -1.483719 & -1.880296 \\ \mathrm{C} & -4.445261 & -0.807227 & 0.295951 \\ \mathrm{H} & -4.342903 & 0.608736 & 1.921570 \\ \mathrm{H} & -4.257983 & -2.151642 & -1.380805 \\ \mathrm{H} & -5.466950 & -1.100588 & 0.516104 \\ \mathrm{H} & 3.086602 & -3.392715 & -0.142453 \\ \mathrm{I} & \text { imaginary frequency } & \\ \mathrm{E}_{\text {chloroform }}=-731.947470 & \\ \mathrm{G}_{\mathrm{chl}} \text { oroform } & =-731.696763 & \\ & & & \end{array}$

\section{TS for 1,2-H shift in oxyallyl cation 6-up in the absence of any anion}

$\begin{array}{lrrr}\mathrm{H} & -3.404948 & 2.400059 & -0.084314 \\ \mathrm{C} & -0.829843 & -0.641561 & 0.289979 \\ \mathrm{C} & -2.040357 & 0.249359 & 0.282150 \\ \mathrm{C} & -1.708511 & 1.523339 & -0.043984 \\ \mathrm{C} & -0.266665 & 1.585082 & -0.311293 \\ \mathrm{C} & 0.320119 & 0.279010 & -0.061276 \\ \mathrm{O} & -2.469408 & 2.625215 & -0.223775 \\ \mathrm{C} & 0.430138 & 2.909207 & -0.481890 \\ \mathrm{H} & -0.083824 & 0.911862 & -1.333657 \\ \mathrm{H} & 1.410230 & 2.804200 & -0.949860 \\ \mathrm{H} & -0.194208 & 3.572761 & -1.083955 \\ \mathrm{H} & 0.555303 & 3.377490 & 0.500395 \\ \mathrm{O} & -3.278805 & -0.214647 & 0.487842 \\ \mathrm{C} & -3.339797 & -1.651054 & 0.679690 \\ \mathrm{C} & -2.583200 & -2.358207 & -0.439238 \\ \mathrm{H} & -2.933340 & -1.888569 & 1.671175 \\ \mathrm{H} & -4.402411 & -1.891394 & 0.670283 \\ \mathrm{C} & -1.084446 & -1.960509 & -0.500455 \\ \mathrm{H} & -3.073144 & -2.105747 & -1.384892 \\ \mathrm{H} & -0.456213 & -2.750872 & -0.084768 \\ \mathrm{H} & -0.787064 & -1.822266 & -1.543451 \\ \mathrm{H} & -0.599861 & -0.910573 & 1.335102\end{array}$




$\begin{array}{lrrr}\mathrm{C} & 1.730830 & -0.060996 & -0.005329 \\ \mathrm{C} & 2.659678 & 0.845744 & 0.549776 \\ \mathrm{C} & 2.188036 & -1.318550 & -0.454593 \\ \mathrm{C} & 4.002772 & 0.501929 & 0.649161 \\ \mathrm{H} & 2.321332 & 1.796215 & 0.943696 \\ \mathrm{C} & 3.534442 & -1.647149 & -0.367753 \\ \mathrm{H} & 1.495083 & -2.023326 & -0.897148 \\ \mathrm{C} & 4.443760 & -0.739273 & 0.184122 \\ \mathrm{H} & 4.705283 & 1.199024 & 1.093933 \\ \mathrm{H} & 3.878770 & -2.610066 & -0.730443 \\ \mathrm{H} & 5.494425 & -1.002136 & 0.255972 \\ \mathrm{H} & -2.681448 & -3.439433 & -0.304998 \\ 1 & \text { imaginary frequency } & \\ \mathrm{E}_{\text {chloroform }}=-731.927061 & \\ \mathrm{G}_{\text {chloroform }}=-731.678496 & \end{array}$

\section{TS for electrocyclization of protonated divinyl ketone $1{ }^{\prime} \mathrm{H}^{+}$in the absence of any anion}

\begin{tabular}{|c|c|c|c|}
\hline $\mathrm{H}$ & 3.377611 & 0.206235 & -1.420696 \\
\hline $\mathrm{C}$ & 0.799407 & 0.796305 & 1.302660 \\
\hline C & 1.451872 & 0.708594 & 0.072515 \\
\hline $\mathrm{C}$ & 2.021362 & -0.553662 & -0.312335 \\
\hline C & 1.447711 & -1.633668 & 0.326135 \\
\hline C & 0.166954 & -1.375855 & 0.936220 \\
\hline 0 & 3.025150 & -0.671237 & -1.187500 \\
\hline $\mathrm{C}$ & 2.097335 & -2.980673 & 0.430003 \\
\hline $\mathrm{H}$ & 3.167850 & -2.922311 & 0.222366 \\
\hline $\mathrm{H}$ & 1.943586 & -3.402898 & 1.430220 \\
\hline $\mathrm{H}$ & 1.646434 & -3.681932 & -0.283449 \\
\hline $\mathrm{O}$ & 1.484976 & 1.679498 & -0.837118 \\
\hline C & 0.910047 & 2.961059 & -0.427812 \\
\hline C & -0.421780 & 2.722722 & 0.268818 \\
\hline $\mathrm{H}$ & 1.636022 & 3.455638 & 0.227059 \\
\hline $\mathrm{H}$ & 0.816205 & 3.521356 & -1.357126 \\
\hline $\mathrm{C}$ & -0.207072 & 1.878487 & 1.536693 \\
\hline $\mathrm{H}$ & -1.093451 & 2.204449 & -0.423944 \\
\hline $\mathrm{H}$ & -0.881827 & 3.683572 & 0.517426 \\
\hline $\mathrm{H}$ & 0.113662 & 2.499389 & 2.382901 \\
\hline $\mathrm{H}$ & -1.155625 & 1.413025 & 1.843251 \\
\hline $\mathrm{H}$ & 1.225218 & 0.290282 & 2.159580 \\
\hline $\mathrm{C}$ & -1.074546 & -1.041501 & 0.250497 \\
\hline C & -1.143207 & -0.823455 & -1.142216 \\
\hline C & -2.253336 & -0.938342 & 1.017812 \\
\hline C & -2.352743 & -0.489077 & -1.735418 \\
\hline $\mathrm{H}$ & -0.256550 & -0.943670 & -1.755091 \\
\hline $\mathrm{C}$ & -3.461884 & -0.599298 & 0.416730 \\
\hline $\mathrm{H}$ & -2.213203 & -1.130236 & 2.086932 \\
\hline $\mathrm{C}$ & -3.512870 & -0.368293 & -0.959080 \\
\hline $\mathrm{H}$ & -2.401948 & -0.340758 & -2.809492 \\
\hline $\mathrm{H}$ & -4.362566 & -0.525003 & 1.017381 \\
\hline $\mathrm{H}$ & -4.456330 & -0.115015 & -1.432161 \\
\hline $\mathrm{H}$ & 0.010416 & -1.858267 & 1.904463 \\
\hline \multicolumn{4}{|c|}{ imaginary frequency } \\
\hline \multicolumn{4}{|c|}{$E_{\text {chloroform }}=-731.939256$} \\
\hline \multicolumn{4}{|c|}{$\mathrm{G}_{\mathrm{chloroform}}=-731.689109$} \\
\hline
\end{tabular}




\section{9-down}

\begin{tabular}{|c|c|c|c|}
\hline $\mathrm{C}$ & -4.520615 & -5.205066 & 1.427148 \\
\hline $\mathrm{C}$ & -4.910458 & -3.819163 & 1.942846 \\
\hline$C$ & -4.515421 & -2.751991 & 0.918169 \\
\hline C & -3.080521 & -2.895943 & 0.438724 \\
\hline C & -2.364691 & -4.086724 & 0.650045 \\
\hline C & -2.997870 & -5.299440 & 1.309219 \\
\hline $\mathrm{C}$ & -2.422247 & -1.804715 & -0.171039 \\
\hline $\mathrm{C}$ & -1.053651 & -1.895444 & -0.438922 \\
\hline C & -0.315556 & -3.064297 & -0.229300 \\
\hline C & -1.016845 & -4.157767 & 0.286636 \\
\hline C & -3.135990 & -0.530018 & -0.473432 \\
\hline C & -4.206125 & -0.469675 & -1.391281 \\
\hline C & -4.897216 & 0.744314 & -1.555434 \\
\hline C & -4.469491 & 1.880952 & -0.865521 \\
\hline C & -3.349246 & 1.878353 & -0.028946 \\
\hline $\mathrm{C}$ & -2.703408 & 0.647628 & 0.145329 \\
\hline C & -4.518879 & -1.652890 & -2.290958 \\
\hline C & -5.935964 & -1.617570 & -2.866601 \\
\hline C & -6.210171 & -0.253904 & -3.503618 \\
\hline $\mathrm{C}$ & -6.123332 & 0.849101 & -2.445526 \\
\hline $\mathrm{O}$ & -0.400973 & -0.734516 & -0.884486 \\
\hline $\mathrm{O}$ & -1.570621 & 0.589956 & 0.963898 \\
\hline$P$ & -0.078166 & 0.369676 & 0.311174 \\
\hline S & 1.202395 & -0.156562 & 1.649837 \\
\hline$S$ & 0.133092 & 2.117245 & -0.818427 \\
\hline $\mathrm{H}$ & -4.983329 & -5.367455 & 0.443530 \\
\hline $\mathrm{H}$ & -4.893096 & -5.995013 & 2.089088 \\
\hline $\mathrm{H}$ & -4.399873 & -3.628680 & 2.896813 \\
\hline $\mathrm{H}$ & -5.987051 & -3.758221 & 2.138642 \\
\hline $\mathrm{H}$ & -5.208572 & -2.817798 & 0.068779 \\
\hline $\mathrm{H}$ & -4.655112 & -1.747894 & 1.333298 \\
\hline $\mathrm{H}$ & -2.707308 & -6.203298 & 0.759415 \\
\hline $\mathrm{H}$ & -2.569302 & -5.410351 & 2.316185 \\
\hline $\mathrm{H}$ & -0.478312 & -5.089002 & 0.440164 \\
\hline $\mathrm{H}$ & -5.005766 & 2.816487 & -1.001923 \\
\hline $\mathrm{H}$ & -3.799618 & -1.626409 & -3.124056 \\
\hline $\mathrm{H}$ & -4.329833 & -2.598447 & -1.781530 \\
\hline $\mathrm{H}$ & -6.056320 & -2.423003 & -3.600180 \\
\hline $\mathrm{H}$ & -6.666631 & -1.801923 & -2.065974 \\
\hline $\mathrm{H}$ & -5.467788 & -0.070602 & -4.292480 \\
\hline $\mathrm{H}$ & -7.195957 & -0.233063 & -3.982314 \\
\hline $\mathrm{H}$ & -6.149524 & 1.838396 & -2.917734 \\
\hline $\mathrm{H}$ & -7.017972 & 0.797241 & -1.806654 \\
\hline $\mathrm{C}$ & -2.817836 & 3.147188 & 0.531760 \\
\hline C & -2.536486 & 4.176386 & -0.350138 \\
\hline C & -1.947946 & 5.382574 & 0.086639 \\
\hline C & -1.638336 & 5.555146 & 1.415148 \\
\hline C & -1.937555 & 4.543543 & 2.363863 \\
\hline C & -1.651154 & 4.719320 & 3.743599 \\
\hline $\mathrm{C}$ & -1.970818 & 3.747002 & 4.661287 \\
\hline $\mathrm{C}$ & -2.604197 & 2.555522 & 4.236019 \\
\hline C & -2.888259 & 2.351651 & 2.905803 \\
\hline C & -2.551726 & 3.323818 & 1.927521 \\
\hline $\mathrm{H}$ & -1.176679 & 5.644626 & 4.060825 \\
\hline $\mathrm{H}$ & -1.745087 & 3.892418 & 5.713809 \\
\hline $\mathrm{H}$ & -2.866292 & 1.795707 & 4.966545 \\
\hline $\mathrm{H}$ & -3.378052 & 1.437365 & 2.592122 \\
\hline
\end{tabular}




\begin{tabular}{|c|c|c|c|}
\hline $\mathrm{H}$ & -1.168477 & 6.472438 & 1.761090 \\
\hline $\mathrm{H}$ & -1.727000 & 6.162383 & -0.636635 \\
\hline $\mathrm{H}$ & -2.725955 & 4.026762 & -1.408551 \\
\hline C & 1.125252 & -3.171677 & -0.570380 \\
\hline C & 1.540321 & -2.894298 & -1.859702 \\
\hline C & 2.876118 & -3.106812 & -2.259831 \\
\hline C & 3.801603 & -3.587295 & -1.364295 \\
\hline C & 3.434079 & -3.847022 & -0.019580 \\
\hline C & 4.383128 & -4.315439 & 0.927785 \\
\hline C & 4.030506 & -4.522004 & 2.241082 \\
\hline C & 2.708149 & -4.252502 & 2.666298 \\
\hline C & 1.760191 & -3.816157 & 1.769184 \\
\hline C & 2.081398 & -3.621288 & 0.398240 \\
\hline $\mathrm{H}$ & 0.823246 & -2.520454 & -2.582378 \\
\hline $\mathrm{H}$ & 3.161679 & -2.898175 & -3.284934 \\
\hline $\mathrm{H}$ & 4.829982 & -3.763305 & -1.668895 \\
\hline $\mathrm{H}$ & 5.402224 & -4.493197 & 0.593799 \\
\hline $\mathrm{H}$ & 4.767452 & -4.875740 & 2.956667 \\
\hline $\mathrm{H}$ & 2.442303 & -4.382223 & 3.711467 \\
\hline $\mathrm{H}$ & 0.757846 & -3.586967 & 2.110253 \\
\hline C & 3.027601 & 2.214170 & -0.427722 \\
\hline C & 1.927674 & 2.189559 & -1.491530 \\
\hline C & 2.299051 & 1.015937 & -2.358099 \\
\hline C & 3.605241 & 0.734007 & -2.243820 \\
\hline C & 4.249447 & 1.606050 & -1.182475 \\
\hline $\mathrm{C}$ & 4.401792 & -0.199064 & -3.094115 \\
\hline $\mathrm{H}$ & 4.892042 & -0.968696 & -2.488119 \\
\hline $\mathrm{H}$ & 3.770223 & -0.686595 & -3.840127 \\
\hline $\mathrm{H}$ & 5.194980 & 0.349595 & -3.621163 \\
\hline $\mathrm{O}$ & 1.862673 & 3.332633 & -2.311673 \\
\hline C & 1.934297 & 4.598956 & -1.632741 \\
\hline C & 3.211437 & 4.709026 & -0.812171 \\
\hline $\mathrm{H}$ & 1.890690 & 5.339972 & -2.434684 \\
\hline $\mathrm{H}$ & 1.050010 & 4.728233 & -0.992129 \\
\hline C & 3.247994 & 3.579449 & 0.221240 \\
\hline $\mathrm{H}$ & 4.074409 & 4.648449 & -1.486990 \\
\hline $\mathrm{H}$ & 2.457427 & 3.747669 & 0.963524 \\
\hline $\mathrm{H}$ & 4.198605 & 3.566794 & 0.766634 \\
\hline C & 5.180364 & 0.872242 & -0.242013 \\
\hline C & 4.779119 & -0.332272 & 0.353246 \\
\hline C & 6.434295 & 1.398481 & 0.080619 \\
\hline C & 5.610754 & -0.985554 & 1.259647 \\
\hline $\mathrm{H}$ & 3.808612 & -0.753261 & 0.108728 \\
\hline C & 7.272508 & 0.744651 & 0.986188 \\
\hline $\mathrm{H}$ & 6.756106 & 2.328958 & -0.382288 \\
\hline $\mathrm{C}$ & 6.860593 & -0.448207 & 1.579200 \\
\hline $\mathrm{H}$ & 5.278384 & -1.912670 & 1.715065 \\
\hline $\mathrm{H}$ & 8.244223 & 1.168096 & 1.226438 \\
\hline $\mathrm{H}$ & 7.509005 & -0.958175 & 2.286714 \\
\hline $\mathrm{H}$ & 3.248921 & 5.688718 & -0.320546 \\
\hline $\mathrm{H}$ & 4.819452 & 2.412738 & -1.668945 \\
\hline $\mathrm{H}$ & 2.751686 & 1.494670 & 0.344702 \\
\hline $\mathrm{O}$ & 1.429208 & 0.473161 & -3.248108 \\
\hline $\mathrm{H}$ & 0.526376 & 0.642020 & -2.922031 \\
\hline \multicolumn{4}{|c|}{0 imaginary frequencies } \\
\hline \multicolumn{4}{|c|}{$E_{\text {chloroform }}=-3563.797381$} \\
\hline & oform $=-356$ & 955993 & \\
\hline
\end{tabular}




\section{TS3-down}

\begin{tabular}{|c|c|c|c|}
\hline & -5.232151 & -2.384015 & 3.723335 \\
\hline & -5.865460 & -1.561129 & 2.600441 \\
\hline & -4.787035 & -0.806286 & 1.819983 \\
\hline & -3.616294 & -1.681796 & 1.403903 \\
\hline & -3.341115 & -2.877587 & 2.088888 \\
\hline & -4.292980 & -3.437393 & 3.131189 \\
\hline & -2.724566 & -1.251165 & 0.397673 \\
\hline & -1.533867 & -1.953394 & 0.188636 \\
\hline & -1.230383 & -3.142681 & 0.865132 \\
\hline & -2.175821 & -3.589552 & 1.792985 \\
\hline & -2.988152 & -0.033631 & -0.423420 \\
\hline & -4.097467 & 0.051636 & -1.293979 \\
\hline & -4.326870 & 1.248542 & -1.993016 \\
\hline & -3.439196 & 2.317326 & -1.837573 \\
\hline & -2.292298 & 2.230484 & -1.047190 \\
\hline & -2.080039 & 1.028824 & -0.362737 \\
\hline & -4.954127 & -1.174494 & -1.565340 \\
\hline & -6.310625 & -0.845100 & -2.192269 \\
\hline & -6.132693 & 0.104093 & -3.378123 \\
\hline & -5.531815 & 1.427560 & -2.899314 \\
\hline & -0.621579 & -1.433580 & -0.728057 \\
\hline & -0.911955 & 0.857114 & 0.382312 \\
\hline & 0.266615 & -0.100083 & -0.324547 \\
\hline & 1.435559 & -0.639892 & 1.262888 \\
\hline & 1.069576 & 0.667668 & -1.915745 \\
\hline & -4.667579 & -1.714683 & 4.387140 \\
\hline & -5.999559 & -2.870177 & 4.336839 \\
\hline & -6.411851 & -2.231897 & 1.921924 \\
\hline & -6.596896 & -0.848917 & 2.999806 \\
\hline & -5.226257 & -0.300690 & 0.957714 \\
\hline & -4.386210 & -0.000129 & 2.453824 \\
\hline & -4.898976 & -4.228138 & 2.663364 \\
\hline & -3.717490 & -3.930038 & 3.924030 \\
\hline & -1.976011 & -4.518378 & 2.320996 \\
\hline & -3.628107 & 3.245718 & -2.370628 \\
\hline & -4.389802 & -1.822476 & -2.253222 \\
\hline & -5.088864 & -1.768380 & -0.659365 \\
\hline & -6.806551 & -1.772170 & -2.502285 \\
\hline & -6.960339 & -0.371886 & -1.441933 \\
\hline & -5.464712 & -0.361011 & -4.115975 \\
\hline & -7.088172 & 0.288625 & -3.882681 \\
\hline & -5.254774 & 2.056714 & -3.753626 \\
\hline & -6.303348 & 1.988246 & -2.349830 \\
\hline & 2.173494 & 3.771183 & -1.601174 \\
\hline & 3.618885 & 1.511289 & 1.141221 \\
\hline & 2.995296 & 2.523769 & 0.420253 \\
\hline & 3.576027 & 2.691020 & -0.895967 \\
\hline & 4.659884 & 1.883219 & -1.001613 \\
\hline & 4.845574 & 1.114357 & 0.299789 \\
\hline & 3.097320 & 3.549403 & -1.825267 \\
\hline & 5.514052 & 1.696656 & -2.207502 \\
\hline & 5.732538 & 1.510684 & 0.818406 \\
\hline & 5.150405 & 2.309389 & -3.036384 \\
\hline & 5.516874 & 0.645920 & -2.521827 \\
\hline & 6.558871 & 1.969649 & -2.005469 \\
\hline & 1.981808 & 3.271742 & 0.824691 \\
\hline & 1.528712 & 2.989344 & 2.185121 \\
\hline
\end{tabular}




\begin{tabular}{|c|c|c|c|}
\hline C & 2.719024 & 2.758756 & 3.112051 \\
\hline $\mathrm{H}$ & 0.867839 & 2.121303 & 2.132543 \\
\hline & 0.950482 & 3.867396 & 2.463730 \\
\hline C & 3.583717 & 1.562893 & 2.660183 \\
\hline & 3.325188 & 3.673111 & 3.122228 \\
\hline & 2.341709 & 2.607571 & 4.129181 \\
\hline & 3.183315 & 0.627645 & 3.073276 \\
\hline $\mathrm{H}$ & 4.599945 & 1.668316 & 3.058201 \\
\hline & 2.546521 & 0.450437 & 0.983935 \\
\hline C & 5.046430 & -0.379307 & 0.117947 \\
\hline & 4.230814 & -1.104628 & -0.760103 \\
\hline C & 6.030345 & -1.056550 & 0.842781 \\
\hline C & 4.394749 & -2.479278 & -0.905519 \\
\hline $\mathrm{H}$ & 3.453987 & -0.592368 & -1.319431 \\
\hline c & 6.196050 & -2.435907 & 0.700904 \\
\hline $\mathrm{F}$ & 6.672309 & -0.501123 & 1.523364 \\
\hline c & 5.378547 & -3.148953 & -0.175083 \\
\hline $\mathrm{H}$ & 3.743598 & -3.027655 & -1.578371 \\
\hline $\mathrm{H}$ & 6.965658 & -2.9 & 1.271756 \\
\hline $\mathrm{F}$ & 5.505930 & -4.221885 & -0.288834 \\
\hline c & -1.352449 & 3.381287 & -0.977713 \\
\hline C & -0.662411 & 3.756313 & -2.112833 \\
\hline C & 0.19 & 4.8 & -2 . \\
\hline C & 0.357090 & 5.630043 & -0.969012 \\
\hline C & -0.349749 & 5.295580 & 0.215072 \\
\hline C & -0.232249 & 6.078205 & 1.393629 \\
\hline C & -0.94 & 5.7 & 2.530134 \\
\hline C & -1.792924 & 4.628056 & 2.536583 \\
\hline C & -1.923465 & 3.845187 & 1.411498 \\
\hline C & -1.213529 & 4.151649 & 0.220137 \\
\hline $\mathrm{H}$ & 0.425991 & 6.9 & 3385 \\
\hline $\mathrm{H}$ & -0.847755 & 6.370313 & 3.422765 \\
\hline $\mathrm{H}$ & -2.348789 & 4.379211 & 3.436119 \\
\hline $\mathrm{H}$ & -2.581436 & 2.983440 & 1.420710 \\
\hline & 1.01 & 6.45 & -0.9 \\
\hline $\mathrm{H}$ & 0.727044 & 5.147225 & -3.022793 \\
\hline $\mathrm{H}$ & -0.766652 & 3.159165 & -3.011833 \\
\hline C & 0.054968 & -3.869730 & 0.702980 \\
\hline C & 0.806647 & -4.11 & 1.838181 \\
\hline C & 2.047446 & -4.784771 & 1.773263 \\
\hline C & 2.537202 & -5.208588 & 0.561953 \\
\hline C & 1.799300 & -4.993438 & -0.629551 \\
\hline C & 2.292132 & -5.430238 & -1.888046 \\
\hline C & 1.568180 & -5.23 & -3.040054 \\
\hline C & 0.304148 & -4.598334 & -2.977114 \\
\hline C & -0.200509 & -4.162878 & -1.773736 \\
\hline C & 0.532738 & -4.324037 & -0.568351 \\
\hline $\mathrm{H}$ & 0.447191 & -3.7493 & 2.794054 \\
\hline $\mathrm{H}$ & 2.618644 & -4.942812 & 2.683311 \\
\hline $\mathrm{H}$ & 3.498654 & -5.710752 & 0.497494 \\
\hline $\mathrm{H}$ & 3.257639 & -5.929622 & -1.921016 \\
\hline $\mathrm{H}$ & 1.958689 & -5.564595 & -3.997189 \\
\hline $\mathrm{H}$ & -0.271080 & -4.454466 & -3.887147 \\
\hline $\mathrm{H}$ & -1.171914 & -3.684971 & -1.739427 \\
\hline \multicolumn{4}{|c|}{1 imaginary frequency } \\
\hline \multirow{2}{*}{\multicolumn{4}{|c|}{$E_{\text {chloroform }}=-3563.771175$}} \\
\hline & & & \\
\hline
\end{tabular}




\section{0-down}

\begin{tabular}{|c|c|c|c|}
\hline & 5.383313 & 1.136774 & 4.015762 \\
\hline & 5.861880 & 0.127095 & 2.971255 \\
\hline & 4.684899 & -0.353866 & 2.119699 \\
\hline 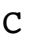 & 3.825190 & 0.778473 & 1.583168 \\
\hline & 3.825753 & 2.036301 & 2.210223 \\
\hline & 4.804163 & 2.371956 & 3.321793 \\
\hline 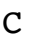 & 2.933497 & 0.549593 & 0.512952 \\
\hline 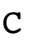 & 1.994076 & 1.531478 & 0.185780 \\
\hline$\tau$ & 1.976340 & 2.795234 & 0.788712 \\
\hline & 2.928320 & 3.021026 & 1.786365 \\
\hline & 2.940996 & -0.716564 & -0.276297 \\
\hline$C$ & 4.054843 & -1.096931 & -1.058543 \\
\hline$C$ & 4.011251 & -2.313322 & -1.760972 \\
\hline C & 2.857308 & -3.101197 & -1.706448 \\
\hline & 1.714911 & -2.711061 & -1.006570 \\
\hline & 1.787252 & -1.504506 & -0.305105 \\
\hline$C$ & 5.225072 & -0.143951 & -1.242925 \\
\hline$C$ & 6.490239 & -0.824470 & -1.770283 \\
\hline & 6.160756 & -1.697370 & -2.981787 \\
\hline & 5.194005 & -2.810754 & -2.572497 \\
\hline 0 & 1.022466 & 1.222581 & -0.769192 \\
\hline b & 0.651584 & -1.040344 & 0.373582 \\
\hline & -0.188640 & 0.183915 & -0.354046 \\
\hline & -1.056701 & 0.915526 & 1.410427 \\
\hline & -1.278077 & -0.230180 & -1.881451 \\
\hline $\mathrm{i}$ & 4.612873 & 0.668640 & 4.643789 \\
\hline $\mathrm{H}$ & 6.201906 & 1.432939 & 4.681677 \\
\hline $\mathrm{I}$ & 6.616688 & 0.599889 & 2.326663 \\
\hline 1 & 6.345610 & -0.732582 & 3.449027 \\
\hline $\mathrm{H}$ & 5.036578 & -0.996538 & 1.309927 \\
\hline $\mathrm{I}$ & 4.036536 & -0.995012 & 2.736473 \\
\hline $\mathrm{H}$ & 5.631140 & 2.957930 & 2.892975 \\
\hline $\mathrm{H}$ & 4.316440 & 3.030289 & 4.050540 \\
\hline $\mathrm{H}$ & 2.950267 & 3.997202 & 2.263759 \\
\hline H & 2.829376 & -4.040726 & -2.252153 \\
\hline $\mathrm{H}$ & 4.908593 & 0.628428 & -1.960588 \\
\hline $\mathrm{H}$ & 5.443300 & 0.396160 & -0.319924 \\
\hline $\mathrm{H}$ & 7.236206 & -0.063166 & -2.025769 \\
\hline H & 6.930786 & -1.449607 & -0.980392 \\
\hline $\mathrm{H}$ & 5.701151 & -1.075038 & -3.761813 \\
\hline $\mathrm{H}$ & 7.069583 & -2.132095 & -3.413422 \\
\hline $\mathrm{H}$ & 4.828958 & -3.349039 & -3.455149 \\
\hline 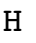 & 5.741859 & -3.552829 & -1.972096 \\
\hline $\mathrm{H}$ & -3.130612 & -2.925447 & -1.746672 \\
\hline C & -4.434154 & -0.850573 & 1.117710 \\
\hline C & -3.859106 & -1.765200 & 0.316449 \\
\hline C & -4.331371 & -1.624342 & -1.065540 \\
\hline C & -5.215214 & -0.605286 & -1.139513 \\
\hline C & -5.349975 & 0.003343 & 0.252585 \\
\hline 0 & -3.912061 & -2.437972 & -2.066971 \\
\hline C & -5.922020 & -0.091439 & -2.349190 \\
\hline $\mathrm{H}$ & -6.388247 & -0.087345 & 0.609781 \\
\hline $\mathrm{H}$ & -5.591424 & -0.629733 & -3.242607 \\
\hline $\mathrm{H}$ & -5.727987 & 0.978870 & -2.504848 \\
\hline $\mathrm{H}$ & -7.012900 & -0.206088 & -2.271138 \\
\hline 0 & -2.968558 & -2.728996 & 0.652222 \\
\hline C & -2.426215 & -2.549336 & 1.980907 \\
\hline
\end{tabular}




\begin{tabular}{|c|c|c|c|}
\hline C & -3.491066 & -2.117023 & 2.992385 \\
\hline & -1.626786 & -1.806488 & 1.915757 \\
\hline & -1.994665 & -3.513710 & 2.244232 \\
\hline & -4.170866 & -0.791310 & 2.586509 \\
\hline & -4.248688 & -2.907288 & 3.061692 \\
\hline & -3.017075 & -2.031643 & 3.977278 \\
\hline & -3.530871 & 0.064371 & 2.849162 \\
\hline & -5.100263 & -0.660194 & 3.158113 \\
\hline & -2.102156 & 1.499720 & 0.774478 \\
\hline & -4.946222 & 1.466962 & 0.342019 \\
\hline & -3.941178 & 1.990966 & -0.485694 \\
\hline & -5.509465 & 2.298304 & 1.315202 \\
\hline & -3.501750 & 3.307228 & -0.330962 \\
\hline & -3.499675 & 1.355556 & -1.246200 \\
\hline & -5.074629 & 3.616321 & 1.470569 \\
\hline & -6.292999 & 1.907482 & 1.960357 \\
\hline & -4.066791 & 4.123931 & 0.651338 \\
\hline $\mathrm{F}$ & -2.711977 & 3.690318 & -0.970764 \\
\hline $\mathrm{H}$ & -5.524987 & 4.24 & 4115 \\
\hline & -3.722755 & 5.145798 & 0.772630 \\
\hline & 0.483484 & -3.545780 & -1.057231 \\
\hline & -0.230145 & -3.619332 & -2.235603 \\
\hline & -1.381413 & -4.4 & -2 . \\
\hline C & -1.804566 & -5.181646 & -1.270713 \\
\hline & -1.087987 & -5.153785 & -0.045943 \\
\hline & -1.486155 & -5.94 & 3581 \\
\hline C & -0.7 & -5 & 401 \\
\hline $\mathrm{C}$ & 0.370890 & -5.096260 & 2.359233 \\
\hline & 0.778925 & -4.312957 & 1.302853 \\
\hline $\mathrm{C}$ & 0.06 & -4.318788 & 2894 \\
\hline $\mathrm{H}$ & -2.3 & -6 & 645 \\
\hline $\mathrm{H}$ & -1.085299 & -6.535038 & 3.080783 \\
\hline $\mathrm{H}$ & 0.928706 & -5.081355 & 3.291341 \\
\hline $\mathrm{H}$ & 1.657873 & $-3.6 \varepsilon$ & 1.397457 \\
\hline $\mathrm{H}$ & -2.6 & -5.8 & -1.3 \\
\hline $\mathrm{H}$ & -1.927453 & -4.463042 & -3.284280 \\
\hline $\mathrm{H}$ & 0.087201 & -3.021533 & -3.083172 \\
\hline C & 0.972553 & & 0.454777 \\
\hline C & 0.21 & 4.3 & 1.481410 \\
\hline C & -0.753254 & 5.370242 & 1.243766 \\
\hline C & -0.962180 & 5.835816 & -0.032439 \\
\hline C & -0.208481 & 5.324688 & -1.120315 \\
\hline C & -0.415 & 5.79 & -2.444186 \\
\hline C & & 5.3191 & -3.490078 \\
\hline C & 1.346472 & 4.355774 & -3.251009 \\
\hline C & 1.567476 & 3.874259 & -1.981593 \\
\hline C & 0.7890 & & -0.881313 \\
\hline $\mathrm{H}$ & 0.343324 & 3.979586 & 2.486154 \\
\hline $\mathrm{H}$ & -1.338140 & 5.756117 & 2.073300 \\
\hline $\mathrm{H}$ & -1.710370 & 6.599493 & -0.229902 \\
\hline $\mathrm{H}$ & -1.182611 & 6.547557 & -2.612482 \\
\hline $\mathrm{H}$ & 0.168799 & 5.684916 & -4.498626 \\
\hline $\mathrm{H}$ & 1.949784 & 3.992650 & -4.077964 \\
\hline $\mathrm{H}$ & 2.347304 & 3.141589 & -1.812956 \\
\hline \multicolumn{4}{|c|}{0 imaginary frequencies } \\
\hline \multicolumn{4}{|c|}{$E_{\text {chloroform }}=-3563.781539$} \\
\hline & form $=-356$ & & \\
\hline
\end{tabular}




\section{TS4'-down}

\begin{tabular}{|c|c|c|c|}
\hline & 4.772123 & 1.064561 & 4.605973 \\
\hline & 5.397320 & -0.039938 & 3.752953 \\
\hline & 4.400449 & -0.529342 & 2.700116 \\
\hline & 3.738378 & 0.595404 & 1.920114 \\
\hline & 3.672422 & 1.891490 & 2.461297 \\
\hline & 4.424810 & 2.267104 & 3.725680 \\
\hline & 3.071823 & 0.324701 & 0.703433 \\
\hline & 2.271499 & 1.320097 & 0.133721 \\
\hline & 2.170171 & 2.612360 & 0.665243 \\
\hline & 2.909858 & 2.872518 & 1.820567 \\
\hline & 3.125746 & -1.021666 & 0.057732 \\
\hline & 4.328579 & -1.596659 & -0.408859 \\
\hline & 4.305076 & -2.907200 & -0.917802 \\
\hline & 3.093831 & -3.603713 & -0.991297 \\
\hline & 1.876875 & -3.029033 & -0.620577 \\
\hline & 1.931104 & -1.725307 & -0.114997 \\
\hline & 5.599651 & -0.766193 & -0.474587 \\
\hline & 6.871380 & -1.608657 & -0.593696 \\
\hline & 6.716032 & -2.646845 & -1.706133 \\
\hline & 5.574652 & -3.608857 & -1.367880 \\
\hline & 1.526832 & 1.004892 & -1.003707 \\
\hline & 0.735803 & -1.083840 & 0.210842 \\
\hline & 0.186230 & 0.061189 & -0.846179 \\
\hline & -1.186631 & 1.032091 & 0.313011 \\
\hline & -0.240299 & -0.585573 & -2.652330 \\
\hline & 3.860993 & 0.679444 & 5.084352 \\
\hline & 5.449980 & 1.373323 & 5.410283 \\
\hline & 6.298022 & 0.349522 & 3.257058 \\
\hline & 5.717233 & -0.883568 & 4.375551 \\
\hline & 4.878648 & -1.246374 & 2.030797 \\
\hline & 3.599867 & -1.094420 & 3.202017 \\
\hline & 5.357561 & 2.776616 & 3.439599 \\
\hline & 3.842006 & 3.003815 & 4.291444 \\
\hline & 2.862359 & 3.870746 & 2.248268 \\
\hline & 3.089229 & -4.620076 & -1.377047 \\
\hline & 5.520155 & -0.114314 & -1.357995 \\
\hline & 5.669702 & -0.086544 & 0.377163 \\
\hline & 7.729989 & -0.954428 & -0.784584 \\
\hline & 7.066963 & -2.121776 & 0.358957 \\
\hline & 6.498678 & -2.131649 & -2.651847 \\
\hline & 7.645742 & -3.208477 & -1.854197 \\
\hline & 5.349857 & -4.255278 & -2.224667 \\
\hline & 5.906195 & -4.281565 & -0.562218 \\
\hline & -2.213625 & -1.422546 & -2.279340 \\
\hline & -5.414988 & -0.395207 & 0.263770 \\
\hline & -4.753153 & -1.444486 & -0.276295 \\
\hline & -3.773879 & -0.975387 & -1.249893 \\
\hline & -3.753440 & 0.422929 & -1.271658 \\
\hline & -4.909338 & 0.883090 & -0.354618 \\
\hline & -3.019542 & -1.853276 & -1.882217 \\
\hline & -3.359188 & 1.299455 & -2.439215 \\
\hline & -5.695072 & 1.295377 & -1.006836 \\
\hline & -2.507063 & 0.892378 & -2.986124 \\
\hline & -3.067099 & 2.292262 & -2.079430 \\
\hline & -4.196823 & 1.422014 & -3.138879 \\
\hline & -4.917481 & -2.755029 & 0.012792 \\
\hline & -5.623654 & -2.952736 & 1.252887 \\
\hline
\end{tabular}




\begin{tabular}{|c|c|c|c|}
\hline & -6.859305 & -2.057891 & 1.366508 \\
\hline $\mathrm{H}$ & -4.921368 & -2.755808 & 2.073962 \\
\hline & -5.889255 & -4.011703 & 1.266351 \\
\hline & -6.466792 & -0.568062 & 1.312263 \\
\hline & -7.537175 & -2.292474 & 0.537387 \\
\hline & -7.387240 & -2.292008 & 2.297515 \\
\hline & -6.099700 & -0.223770 & 2.289658 \\
\hline & -7.344483 & 0.052915 & 1.089904 \\
\hline & -2.478170 & 0.599214 & -0.462438 \\
\hline & -4.521241 & 1.969027 & 0.634008 \\
\hline & -3.907379 & 1.653670 & 1.852909 \\
\hline & -4.728013 & 3.315041 & 0.314974 \\
\hline & -3.505222 & 2.661568 & 2.728190 \\
\hline & -3.725951 & 0.613530 & 2.105898 \\
\hline & -4.328473 & 4.325878 & 1.189041 \\
\hline & -5.204772 & 3.571793 & -0.627835 \\
\hline & -3.716576 & 4.001384 & 2.400169 \\
\hline & -3.021838 & 2.397974 & 3.664521 \\
\hline & -4.496937 & 5.365997 & 0.923832 \\
\hline & -3.407854 & 4.786714 & 3.084562 \\
\hline & 0.583084 & -3.726354 & -0.832450 \\
\hline C & 0.267226 & -4.16 & -2.106148 \\
\hline$c$ & -0.978957 & -4.759792 & -2.392877 \\
\hline & -1.913429 & -4.916635 & -1.397906 \\
\hline$c$ & -1.625028 & -4.515090 & -0.069013 \\
\hline C & -2.568627 & -4.698770 & 0.975471 \\
\hline C & -2.277058 & -4.328557 & 2.266967 \\
\hline C & -1.016384 & -3.761258 & 2.570178 \\
\hline C & -0.082199 & -3.566904 & 1.579508 \\
\hline C & -0.356123 & -3.921925 & 0.231506 \\
\hline $\mathrm{H}$ & -3.528485 & -5.142226 & 0.727804 \\
\hline $\mathrm{H}$ & -3.005414 & -4.481698 & 3.059072 \\
\hline $\mathrm{H}$ & -0.784222 & -3.480470 & 3.593730 \\
\hline $\mathrm{H}$ & 0.8 & -3.1 & 1.824935 \\
\hline $\mathrm{H}$ & -2.889825 & -5.341655 & -1.613191 \\
\hline $\mathrm{H}$ & -1.202712 & -5.066326 & -3.410551 \\
\hline $\mathrm{H}$ & & -3.987139 & -2.909755 \\
\hline c & & 3.6 & 1330 \\
\hline C & 0.256120 & 4.140732 & 0.922365 \\
\hline $\mathrm{C}$ & -0.710418 & 5.046064 & 0.435795 \\
\hline C & -0.684622 & 5.43 & -0.880617 \\
\hline C & & 4.9 & -1.756304 \\
\hline C & 0.382204 & 5.379446 & -3.113400 \\
\hline $\mathrm{C}$ & 1.377127 & 4.931310 & -3.949807 \\
\hline C & 2.369451 & 4.05 & -3.458111 \\
\hline C & 2.339867 & 3.62 & -2.150144 \\
\hline C & 1.316972 & 4.053996 & -1.263261 \\
\hline $\mathrm{H}$ & 0.190140 & 3.790143 & 1.947553 \\
\hline $\mathrm{H}$ & -1.491914 & 5.398292 & 1.100613 \\
\hline $\mathrm{H}$ & -1.436790 & 6.119938 & -1.271485 \\
\hline $\mathrm{H}$ & -0.379362 & 6.064794 & -3.477685 \\
\hline $\mathrm{H}$ & 1.407756 & 5.254714 & -4.986513 \\
\hline $\mathrm{H}$ & 3.158813 & 3.706418 & -4.120106 \\
\hline $\mathrm{H}$ & 3.106641 & 2.954031 & -1.782699 \\
\hline \multicolumn{4}{|c|}{1 imaginary frequency } \\
\hline \multicolumn{4}{|c|}{$\mathrm{E}_{\text {chloroform }}=-3563.766010$} \\
\hline & roform $=-356$ & 932450 & \\
\hline
\end{tabular}




\section{Molecular Dynamics Simulations}

\section{Topologies generated by LigParGen for the anion and cation of 7}

\begin{tabular}{|c|c|c|c|c|c|c|c|}
\hline \multicolumn{8}{|l|}{ Anion } \\
\hline \multicolumn{8}{|c|}{ 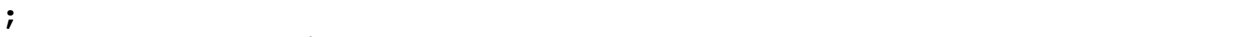 } \\
\hline \multicolumn{8}{|c|}{; GENERATED BY LigParGen Server } \\
\hline \multicolumn{8}{|c|}{; Jorgensen Lab @ Yale University } \\
\hline \multicolumn{8}{|c|}{ type $]$} \\
\hline \multirow{2}{*}{\multicolumn{8}{|c|}{$\begin{array}{l}\text { [ molecule } \\
\text {; Name }\end{array}$}} \\
\hline & & & & & & & \\
\hline \multirow{2}{*}{\multicolumn{8}{|c|}{$\begin{array}{l}\text { ANI } \\
{[\text { at }}\end{array}$}} \\
\hline & & & & & & & \\
\hline $\mathrm{nr}$ & type & resnr & residue & atom & cgnr & charge & mass \\
\hline 1 & opls_800 & 1 & ANI & $\mathrm{C} 00$ & 1 & -0.1532 & 12.0110 \\
\hline 2 & opls_801 & 1 & ANI & $\mathrm{C} 01$ & 1 & -0.1543 & 12.0110 \\
\hline 3 & opls 802 & 1 & ANI & $\mathrm{C} 02$ & 1 & -0.1166 & 12.0110 \\
\hline 4 & opls 803 & 1 & ANI & $\mathrm{C} 03$ & 1 & -0.0211 & 12.0110 \\
\hline 5 & opls 804 & 1 & ANI & $\mathrm{C} 04$ & 1 & -0.0963 & 12.0110 \\
\hline 6 & opls-805 & 1 & ANI & $\mathrm{C} 05$ & 1 & -0.1098 & 12.0110 \\
\hline 7 & opls_806 & 1 & ANI & $\mathrm{C} 06$ & 1 & -0.0821 & 12.0110 \\
\hline 8 & opls_807 & 1 & ANI & $\mathrm{C} 07$ & 1 & 0.1689 & 12.0110 \\
\hline 9 & opls_808 & 1 & ANI & $\mathrm{C} 08$ & 1 & -0.0251 & 12.0110 \\
\hline 10 & opls_809 & 1 & ANI & $\mathrm{C} 09$ & 1 & -0.1052 & 12.0110 \\
\hline 11 & opls_810 & 1 & ANI & $\mathrm{COA}$ & 1 & -0.0638 & 12.0110 \\
\hline 12 & opls_811 & 1 & ANI & $\mathrm{COB}$ & 1 & -0.0224 & 12.0110 \\
\hline 13 & opls_812 & 1 & ANI & $\mathrm{COC}$ & 1 & -0.0904 & 12.0110 \\
\hline 14 & opls_813 & 1 & ANI & COD & 1 & -0.1071 & 12.0110 \\
\hline 15 & opls_814 & 1 & ANI & $\mathrm{COE}$ & 1 & -0.0217 & 12.0110 \\
\hline 16 & opls_815 & 1 & ANI & $\mathrm{COF}$ & 1 & 0.1836 & 12.0110 \\
\hline 17 & opls_816 & 1 & ANI & $\mathrm{COG}$ & 1 & -0.1164 & 12.0110 \\
\hline 18 & opls 817 & 1 & ANI & $\mathrm{COH}$ & 1 & -0.1542 & 12.0110 \\
\hline 19 & opls 818 & 1 & ANI & $\mathrm{COI}$ & 1 & -0.1531 & 12.0110 \\
\hline 20 & opls_819 & 1 & ANI & $\mathrm{COJ}$ & 1 & -0.1109 & 12.0110 \\
\hline 21 & opls_820 & 1 & ANI & $\mathrm{OOK}$ & 1 & -0.7202 & 15.9990 \\
\hline 22 & opls_821 & 1 & ANI & $\mathrm{OOM}$ & 1 & -0.7514 & 15.9990 \\
\hline 23 & opls_822 & 1 & ANI & PON & 1 & 2.5245 & 30.9738 \\
\hline 24 & opls_823 & 1 & ANI & S00 & 1 & -1.1755 & 32.0600 \\
\hline 25 & opls_824 & 1 & ANI & SOP & 1 & -1.1872 & 32.0600 \\
\hline 26 & opls_825 & 1 & ANI & $\mathrm{HOQ}$ & 1 & 0.0767 & 1.0080 \\
\hline 27 & opls_826 & 1 & ANI & HOR & 1 & 0.0767 & 1.0080 \\
\hline 28 & opls_827 & 1 & ANI & HOS & 1 & 0.0749 & 1.0080 \\
\hline 29 & opls_828 & 1 & ANI & Нот & 1 & 0.0749 & 1.0080 \\
\hline 30 & opls_829 & 1 & ANI & $\mathrm{HOU}$ & 1 & 0.0926 & 1.0080 \\
\hline 31 & opls-830 & 1 & ANI & $\mathrm{HOV}$ & 1 & 0.0926 & 1.0080 \\
\hline 32 & opls_831 & 1 & ANI & HOW & 1 & 0.0860 & 1.0080 \\
\hline 33 & opls_832 & 1 & ANI & HOX & 2 & 0.0860 & 1.0080 \\
\hline 34 & opls_833 & 1 & ANI & HOY & 2 & 0.1346 & 1.0080 \\
\hline 35 & opls_834 & 1 & ANI & $\mathrm{HOZ}$ & 2 & 0.1375 & 1.0080 \\
\hline 36 & opls_835 & 1 & ANI & $\mathrm{H} 10$ & 2 & 0.0956 & 1.0080 \\
\hline 37 & opls_836 & 1 & ANI & $\mathrm{H} 11$ & 2 & 0.0956 & 1.0080 \\
\hline 38 & opls_837 & 1 & ANI & $\mathrm{H} 12$ & 2 & 0.0760 & 1.0080 \\
\hline 39 & opls_838 & 1 & ANI & $\mathrm{H} 13$ & 2 & 0.0760 & 1.0080 \\
\hline 40 & opls_839 & 1 & ANI & $\mathrm{H} 14$ & 2 & 0.0771 & 1.0080 \\
\hline 41 & opls_840 & 1 & ANI & H15 & 2 & 0.0771 & 1.0080 \\
\hline 42 & opls_841 & 1 & ANI & $\mathrm{H} 16$ & 2 & 0.0865 & 1.0080 \\
\hline 43 & opls_842 & 1 & ANI & $\mathrm{H} 17$ & 2 & 0.0865 & 1.0080 \\
\hline 44 & opls 843 & 1 & ANI & $\mathrm{C} 18$ & 2 & -0.0385 & 12.0110 \\
\hline 45 & opls- 844 & 1 & ANI & C19 & 2 & -0.0832 & 12.0110 \\
\hline 46 & opls_845 & 1 & ANI & $\mathrm{C} 1 \mathrm{~A}$ & 2 & -0.1224 & 12.0110 \\
\hline 47 & opls_846 & 1 & ANI & $\mathrm{C} 1 \mathrm{~B}$ & 2 & -0.1145 & 12.0110 \\
\hline 48 & opls 847 & 1 & ANI & $\mathrm{C} 1 \mathrm{C}$ & 2 & -0.0294 & 12.0110 \\
\hline
\end{tabular}




\begin{tabular}{|c|c|c|c|c|c|c|}
\hline 49 & opls_848 & AN & C1D & 2 & -0.1214 & 12.0110 \\
\hline 50 & opls_849 & $\mathrm{AN}$ & $\mathrm{C} 1 \mathrm{E}$ & 2 & -0.1339 & 12.0110 \\
\hline 51 & opls_850 & AN & $\mathrm{C} 1 \mathrm{~F}$ & 2 & -0.1322 & 12.0110 \\
\hline 52 & opls_851 & AN & $\mathrm{C} 1 \mathrm{G}$ & 2 & -0.1027 & 12.0110 \\
\hline 53 & opls 852 & AN & $\mathrm{C} 1 \mathrm{H}$ & 2 & 0.0075 & 12.0110 \\
\hline 54 & opls 853 & AN & $\mathrm{H} 1 \mathrm{I}$ & 2 & 0.1221 & 1.0080 \\
\hline 55 & opls 854 & AN & $\mathrm{H} 1 \mathrm{~J}$ & 2 & 0.1198 & 1.0080 \\
\hline 56 & opls_855 & AN & $\mathrm{H} 1 \mathrm{~K}$ & 2 & 0.1236 & 1.0080 \\
\hline 57 & opls_856 & AN & $\mathrm{H} 1 \mathrm{M}$ & 2 & 0.1319 & 1.0080 \\
\hline 58 & opls_857 & AN & $\mathrm{H} 1 \mathrm{~N}$ & 2 & 0.1225 & 1.0080 \\
\hline 59 & opls_858 & AN & $\mathrm{H} 1 \mathrm{O}$ & 2 & 0.1322 & 1.0080 \\
\hline 60 & opls_859 & AN & $\mathrm{H} 1 \mathrm{P}$ & 2 & 0.1483 & 1.0080 \\
\hline 61 & opls_860 & AN & $\mathrm{C} 1 \mathrm{Q}$ & 2 & -0.0343 & 12.0110 \\
\hline 62 & opls_861 & AN & C1R & 2 & -0.0807 & 12.0110 \\
\hline 63 & opls_862 & AN & $\mathrm{C} 1 \mathrm{~S}$ & 2 & -0.1207 & 12.0110 \\
\hline 64 & opls_863 & AN & $\mathrm{C} 1 \mathrm{~T}$ & 2 & -0.1167 & 12.0110 \\
\hline 65 & opls_864 & AN & $\mathrm{C} 1 \mathrm{U}$ & 2 & -0.0293 & 12.0110 \\
\hline 66 & opls_865 & AN & $\mathrm{C} 1 \mathrm{~V}$ & 3 & -0.1231 & 12.0110 \\
\hline 67 & opls_866 & AN & $\mathrm{C} 1 \mathrm{~W}$ & 3 & -0.1347 & 12.0110 \\
\hline 68 & opls_867 & AN & $\mathrm{C} 1 \mathrm{x}$ & 3 & -0.1361 & 12.0110 \\
\hline 69 & opls 868 & AN & $\mathrm{C} 1 \mathrm{Y}$ & 3 & -0.0979 & 12.0110 \\
\hline 70 & opls_869 & AN & $\mathrm{C} 1 \mathrm{Z}$ & 3 & 0.0075 & 12.0110 \\
\hline 71 & opls_870 & AN & $\mathrm{H} 20$ & 3 & 0.1482 & 1.0080 \\
\hline 72 & opls_871 & AN & H2 1 & 3 & 0.1311 & 1.0080 \\
\hline 73 & opls_872 & AN & H22 & 3 & 0.1211 & 1.0080 \\
\hline 74 & opls_873 & AN & H23 & 3 & 0.1209 & 1.0080 \\
\hline 75 & opls_874 & AN & H2 4 & 3 & 0.1182 & 1.0080 \\
\hline 76 & opls_875 & AN & H25 & 3 & 0.1221 & 1.0080 \\
\hline 77 & opls_876 & AN & H26 & 3 & 0.1329 & 1.0080 \\
\hline [ bonds & ] - & & & & & \\
\hline 2 & 1 & 0.1529 & 224262.400 & & & \\
\hline 3 & 2 & 0.1529 & 224262.400 & & & \\
\hline 4 & 3 & 0.1510 & 265265.600 & & & \\
\hline 5 & 4 & 0.1400 & 392459.200 & & & \\
\hline 6 & 1 & 0.1529 & 224262.400 & & & \\
\hline 7 & 4 & 0.1400 & 392459.200 & & & \\
\hline 8 & 7 & 0.1400 & 392459.200 & & & \\
\hline 9 & 8 & 0.1400 & 392459.200 & & & \\
\hline 10 & 5 & 0.1400 & 392459.200 & & & \\
\hline 11 & 7 & 0.1400 & 392459.200 & & & \\
\hline 12 & 11 & 0.1400 & 392459.200 & & & \\
\hline 13 & 12 & 0.1400 & 392459.200 & & & \\
\hline 14 & 13 & 0.1400 & 392459.200 & & & \\
\hline 15 & 14 & 0.1400 & 392459.200 & & & \\
\hline 16 & 11 & 0.1400 & 392459.200 & & & \\
\hline 17 & 12 & 0.1510 & 265265.600 & & & \\
\hline 18 & 17 & 0.1529 & 224262.400 & & & \\
\hline 19 & 18 & 0.1529 & 224262.400 & & & \\
\hline 20 & 13 & 0.1510 & 265265.600 & & & \\
\hline 21 & 8 & 0.1364 & 376560.000 & & & \\
\hline 22 & 16 & 0.1364 & 376560.000 & & & \\
\hline 23 & 21 & 0.1610 & 192464.000 & & & \\
\hline 24 & 23 & 0.1930 & 136155.728 & & & \\
\hline 25 & 23 & 0.1930 & 136155.728 & & & \\
\hline 26 & 1 & 0.1090 & 284512.000 & & & \\
\hline 27 & 1 & 0.1090 & 284512.000 & & & \\
\hline 28 & 2 & 0.1090 & 284512.000 & & & \\
\hline 29 & 2 & 0.1090 & 284512.000 & & & \\
\hline 30 & 3 & 0.1090 & 284512.000 & & & \\
\hline 31 & 3 & 0.1090 & 284512.000 & & & \\
\hline 32 & 6 & 0.1090 & 284512.000 & & & \\
\hline 33 & 6 & 0.1090 & 284512.000 & & & \\
\hline 34 & 10 & 0.1080 & 307105.600 & & & \\
\hline 35 & 14 & 0.1080 & 307105.600 & & & \\
\hline 36 & 17 & 0.1090 & 284512.000 & & & \\
\hline
\end{tabular}




\begin{tabular}{|c|c|c|c|c|}
\hline 37 & 17 & 1 & 0.1090 & 284512.000 \\
\hline 38 & 18 & 1 & 0.1090 & 284512.000 \\
\hline 39 & 18 & 1 & 0.1090 & 284512.000 \\
\hline 40 & 19 & 1 & 0.1090 & 284512.000 \\
\hline 41 & 19 & 1 & 0.1090 & 284512.000 \\
\hline 42 & 20 & 1 & 0.1090 & 284512.000 \\
\hline 43 & 20 & 1 & 0.1090 & 284512.000 \\
\hline 44 & 15 & 1 & 0.1460 & 322168.000 \\
\hline 45 & 44 & 1 & 0.1400 & 392459.200 \\
\hline 46 & 45 & 1 & 0.1400 & 392459.200 \\
\hline 47 & 46 & 1 & 0.1400 & 392459.20 \\
\hline 48 & 47 & 1 & 0.1404 & 392459.200 \\
\hline 49 & 48 & 1 & 0.1404 & 392459.200 \\
\hline 50 & 49 & 1 & 0.1400 & 392459.200 \\
\hline 51 & 50 & 1 & 0.1400 & 392459.200 \\
\hline 52 & 51 & 1 & 0.1400 & 392459.200 \\
\hline 53 & 44 & 1 & 0.1400 & 392459.200 \\
\hline 54 & 49 & 1 & 0.1080 & 307105.600 \\
\hline 55 & 50 & 1 & 0.1080 & 307105.600 \\
\hline 56 & 51 & 1 & 0.1080 & 307105.600 \\
\hline 57 & 52 & 1 & 0.1080 & 307105.600 \\
\hline 58 & 47 & 1 & 0.1080 & 307105.600 \\
\hline 59 & 46 & 1 & 0.1080 & 307105.600 \\
\hline 60 & 45 & 1 & 0.1080 & 307105.600 \\
\hline 61 & 9 & 1 & 0.1460 & 322168.00 \\
\hline 62 & 61 & 1 & 0.1400 & 392459.20 \\
\hline 63 & 62 & 1 & 0.1400 & 392459.20 \\
\hline 64 & 63 & 1 & 0.1400 & 392459.20 \\
\hline 65 & 64 & 1 & 0.1404 & 392459.200 \\
\hline 66 & 65 & 1 & 0.1404 & 392459.200 \\
\hline 67 & 66 & 1 & 0.1400 & 392459.200 \\
\hline 68 & 67 & 1 & 0.1400 & 392459.200 \\
\hline 69 & 68 & 1 & 0.1400 & 392459.200 \\
\hline 70 & 61 & 1 & 0.1400 & 392459.200 \\
\hline 71 & 62 & 1 & 0.1080 & 307105.600 \\
\hline 72 & 63 & 1 & 0.1080 & 307105.60 \\
\hline 73 & 64 & 1 & 0.1080 & 307105.60 \\
\hline 74 & 66 & 1 & 0.1080 & 307105.60 \\
\hline 75 & 67 & 1 & 0.1080 & 307105.60 \\
\hline 76 & 68 & 1 & 0.1080 & 307105.60 \\
\hline 77 & 69 & 1 & 0.1080 & 307105.60 \\
\hline 6 & 5 & 1 & 0.1510 & 265265.60 \\
\hline 10 & 9 & 1 & 0.1400 & 392459.200 \\
\hline 16 & 15 & 1 & 0.1400 & 392459.200 \\
\hline 20 & 19 & 1 & 0.1529 & 224262.400 \\
\hline 23 & 22 & 1 & 0.1610 & 192464.00 \\
\hline 53 & 48 & 1 & 0.1370 & 435136.00 \\
\hline 53 & 52 & 1 & 0.1404 & 392459.20 \\
\hline 70 & 65 & 1 & 0.1370 & 435136.00 \\
\hline 70 & 69 & 1 & 0.1404 & 392459.200 \\
\hline
\end{tabular}

[ angles ]

\begin{tabular}{rrrrrr}
$i \quad \mathrm{ai}$ & $\mathrm{aj}$ & ak & funct & \multicolumn{3}{c}{$\mathrm{c} 0$} \\
1 & 2 & 3 & 1 & 112.700 & 488.273 \\
2 & 3 & 4 & 1 & 114.000 & 527.184 \\
3 & 4 & 5 & 1 & 120.000 & 585.760 \\
2 & 1 & 6 & 1 & 112.700 & 488.273 \\
3 & 4 & 7 & 1 & 120.000 & 585.760 \\
4 & 7 & 8 & 1 & 120.000 & 527.184 \\
7 & 8 & 9 & 1 & 120.000 & 527.184 \\
4 & 5 & 10 & 1 & 120.000 & 527.184 \\
4 & 7 & 11 & 1 & 120.000 & 527.184 \\
7 & 11 & 12 & 1 & 120.000 & 527.184 \\
11 & 12 & 13 & 1 & 120.000 & 527.184 \\
12 & 13 & 14 & 1 & 120.000 & 527.184
\end{tabular}

c1

c2

c3 


\begin{tabular}{|c|c|c|c|c|c|}
\hline 13 & 14 & 15 & 1 & 120.000 & 527.184 \\
\hline 7 & 11 & 16 & 1 & 120.000 & 527.184 \\
\hline 11 & 12 & 17 & 1 & 120.000 & 585.760 \\
\hline 12 & 17 & 18 & 1 & 114.000 & 527.184 \\
\hline 17 & 18 & 19 & 1 & 112.700 & 488.273 \\
\hline 12 & 13 & 20 & 1 & 120.000 & 585.760 \\
\hline 7 & 8 & 21 & 1 & 120.000 & 585.760 \\
\hline 11 & 16 & 22 & 1 & 120.000 & 585.760 \\
\hline 8 & 21 & 23 & 1 & 120.500 & 836.800 \\
\hline 21 & 23 & 24 & 1 & 108.400 & 531.954 \\
\hline 21 & 23 & 25 & 1 & 108.400 & 531.954 \\
\hline 2 & 1 & 26 & 1 & 110.700 & 313.800 \\
\hline 2 & 1 & 27 & 1 & 110.700 & 313.800 \\
\hline 1 & 2 & 28 & 1 & 110.700 & 313.800 \\
\hline 1 & 2 & 29 & 1 & 110.700 & 313.800 \\
\hline 2 & 3 & 30 & 1 & 110.700 & 313.800 \\
\hline 2 & 3 & 31 & 1 & 110.700 & 313.800 \\
\hline 1 & 6 & 32 & 1 & 110.700 & 313.800 \\
\hline 1 & 6 & 33 & 1 & 110.700 & 313.800 \\
\hline 5 & 10 & 34 & 1 & 120.000 & 292.880 \\
\hline 13 & 14 & 35 & 1 & 120.000 & 292.880 \\
\hline 12 & 17 & 36 & 1 & 109.500 & 292.880 \\
\hline 12 & 17 & 37 & 1 & 109.500 & 292.880 \\
\hline 17 & 18 & 38 & 1 & 110.700 & 313.800 \\
\hline 17 & 18 & 39 & 1 & 110.700 & 313.800 \\
\hline 18 & 19 & 40 & 1 & 110.700 & 313.800 \\
\hline 18 & 19 & 41 & 1 & 110.700 & 313.800 \\
\hline 13 & 20 & 42 & 1 & 109.500 & 292.880 \\
\hline 13 & 20 & 43 & 1 & 109.500 & 292.880 \\
\hline 14 & 15 & 44 & 1 & 120.000 & 527.184 \\
\hline 15 & 44 & 45 & 1 & 120.000 & 527.184 \\
\hline 44 & 45 & 46 & 1 & 120.000 & 527.184 \\
\hline 45 & 46 & 47 & 1 & 120.000 & 527.184 \\
\hline 46 & 47 & 48 & 1 & 120.000 & 527.184 \\
\hline 47 & 48 & 49 & 1 & 134.900 & 711.280 \\
\hline 48 & 49 & 50 & 1 & 120.000 & 527.184 \\
\hline 49 & 50 & 51 & 1 & 120.000 & 527.184 \\
\hline 50 & 51 & 52 & 1 & 120.000 & 527.184 \\
\hline 15 & 44 & 53 & 1 & 120.000 & 527.184 \\
\hline 48 & 49 & 54 & 1 & 120.000 & 292.880 \\
\hline 49 & 50 & 55 & 1 & 120.000 & 292.880 \\
\hline 50 & 51 & 56 & 1 & 120.000 & 292.880 \\
\hline 51 & 52 & 57 & 1 & 120.000 & 292.880 \\
\hline 46 & 47 & 58 & 1 & 120.000 & 292.880 \\
\hline 45 & 46 & 59 & 1 & 120.000 & 292.880 \\
\hline 44 & 45 & 60 & 1 & 120.000 & 292.880 \\
\hline 8 & 9 & 61 & 1 & 120.000 & 527.184 \\
\hline 9 & 61 & 62 & 1 & 120.000 & 527.184 \\
\hline 61 & 62 & 63 & 1 & 120.000 & 527.184 \\
\hline 62 & 63 & 64 & 1 & 120.000 & 527.184 \\
\hline 63 & 64 & 65 & 1 & 120.000 & 527.184 \\
\hline 64 & 65 & 66 & 1 & 134.900 & 711.280 \\
\hline 65 & 66 & 67 & 1 & 120.000 & 527.184 \\
\hline 66 & 67 & 68 & 1 & 120.000 & 527.184 \\
\hline 67 & 68 & 69 & 1 & 120.000 & 527.184 \\
\hline 9 & 61 & 70 & 1 & 120.000 & 527.184 \\
\hline 61 & 62 & 71 & 1 & 120.000 & 292.880 \\
\hline 62 & 63 & 72 & 1 & 120.000 & 292.880 \\
\hline 63 & 64 & 73 & 1 & 120.000 & 292.880 \\
\hline 65 & 66 & 74 & 1 & 120.000 & 292.880 \\
\hline 66 & 67 & 75 & 1 & 120.000 & 292.880 \\
\hline 67 & 68 & 76 & 1 & 120.000 & 292.880 \\
\hline 68 & 69 & 77 & 1 & 120.000 & 292.880 \\
\hline 22 & 23 & 25 & 1 & 108.400 & 531.954 \\
\hline 5 & 4 & 7 & 1 & 120.000 & 527.184 \\
\hline
\end{tabular}




\begin{tabular}{|c|c|c|c|c|c|}
\hline 48 & 47 & 58 & 1 & 120.000 & 292.880 \\
\hline 28 & 2 & 29 & 1 & 107.800 & 276.144 \\
\hline 51 & 52 & 53 & 1 & 120.000 & 527.184 \\
\hline 5 & 10 & 9 & 1 & 120.000 & 527.184 \\
\hline 47 & 46 & 59 & 1 & 120.000 & 292.880 \\
\hline 61 & 70 & 69 & 1 & 134.900 & 711.280 \\
\hline 19 & 20 & 43 & 1 & 110.700 & 313.800 \\
\hline 13 & 12 & 17 & 1 & 120.000 & 585.760 \\
\hline 19 & 18 & 38 & 1 & 110.700 & 313.800 \\
\hline 16 & 22 & 23 & 1 & 120.500 & 836.800 \\
\hline 53 & 52 & 57 & 1 & 120.000 & 292.880 \\
\hline 1 & 6 & 5 & 1 & 114.000 & 527.184 \\
\hline 65 & 64 & 73 & 1 & 120.000 & 292.880 \\
\hline 22 & 23 & 24 & 1 & 108.400 & 531.954 \\
\hline 64 & 63 & 72 & 1 & 120.000 & 292.880 \\
\hline 70 & 69 & 77 & 1 & 120.000 & 292.880 \\
\hline 18 & 19 & 20 & 1 & 112.700 & 488.273 \\
\hline 9 & 10 & 34 & 1 & 120.000 & 292.880 \\
\hline 15 & 16 & 22 & 1 & 120.000 & 585.760 \\
\hline 68 & 69 & 70 & 1 & 120.000 & 527.184 \\
\hline 6 & 1 & 27 & 1 & 110.700 & 313.800 \\
\hline 16 & 15 & 44 & 1 & 120.000 & 527.184 \\
\hline 44 & 53 & 52 & 1 & 134.900 & 711.280 \\
\hline 20 & 19 & 41 & 1 & 110.700 & 313.800 \\
\hline 6 & 5 & 10 & 1 & 120.000 & 585.760 \\
\hline 65 & 70 & 69 & 1 & 117.300 & 711.280 \\
\hline 67 & 66 & 74 & 1 & 120.000 & 292.880 \\
\hline 18 & 17 & 36 & 1 & 110.700 & 313.800 \\
\hline 5 & 6 & 33 & 1 & 109.500 & 292.880 \\
\hline 48 & 53 & 52 & 1 & 117.300 & 711.280 \\
\hline 51 & 50 & 55 & 1 & 120.000 & 292.880 \\
\hline 38 & 18 & 39 & 1 & 107.800 & 276.144 \\
\hline 69 & 68 & 76 & 1 & 120.000 & 292.880 \\
\hline 13 & 20 & 19 & 1 & 114.000 & 527.184 \\
\hline 68 & 67 & 75 & 1 & 120.000 & 292.880 \\
\hline 49 & 48 & 53 & 1 & 117.300 & 711.280 \\
\hline 62 & 61 & 70 & 1 & 120.000 & 527.184 \\
\hline 40 & 19 & 41 & 1 & 107.800 & 276.144 \\
\hline 10 & 9 & 61 & 1 & 120.000 & 527.184 \\
\hline 3 & 2 & 28 & 1 & 110.700 & 313.800 \\
\hline 9 & 8 & 21 & 1 & 120.000 & 585.760 \\
\hline 19 & 18 & 39 & 1 & 110.700 & 313.800 \\
\hline 11 & 16 & 15 & 1 & 120.000 & 527.184 \\
\hline 24 & 23 & 25 & 1 & 108.400 & 531.954 \\
\hline 3 & 2 & 29 & 1 & 110.700 & 313.800 \\
\hline 8 & 7 & 11 & 1 & 120.000 & 527.184 \\
\hline 20 & 19 & 40 & 1 & 110.700 & 313.800 \\
\hline 19 & 20 & 42 & 1 & 110.700 & 313.800 \\
\hline 6 & 1 & 26 & 1 & 110.700 & 313.800 \\
\hline 32 & 6 & 33 & 1 & 107.800 & 276.144 \\
\hline 45 & 44 & 53 & 1 & 120.000 & 527.184 \\
\hline 44 & 53 & 48 & 1 & 117.300 & 711.280 \\
\hline 21 & 23 & 22 & 1 & 102.600 & 376.560 \\
\hline 64 & 65 & 70 & 1 & 117.300 & 711.280 \\
\hline 36 & 17 & 37 & 1 & 107.800 & 276.144 \\
\hline 14 & 13 & 20 & 1 & 120.000 & 585.760 \\
\hline 18 & 17 & 37 & 1 & 110.700 & 313.800 \\
\hline 63 & 62 & 71 & 1 & 120.000 & 292.880 \\
\hline 47 & 48 & 53 & 1 & 117.300 & 711.280 \\
\hline 61 & 70 & 65 & 1 & 117.300 & 711.280 \\
\hline 4 & 3 & 31 & 1 & 109.500 & 292.880 \\
\hline 14 & 15 & 16 & 1 & 120.000 & 527.184 \\
\hline 15 & 14 & 35 & 1 & 120.000 & 292.880 \\
\hline 42 & 20 & 43 & 1 & 107.800 & 276.144 \\
\hline 8 & 9 & 10 & 1 & 120.000 & 527.184 \\
\hline
\end{tabular}




\begin{tabular}{|c|c|c|c|c|c|c|c|c|c|c|c|c|c|}
\hline & 5 & 6 & 32 & & 1 & 109.500 & \multicolumn{2}{|c|}{292.880} & & & & & \\
\hline & 4 & 3 & 30 & & 1 & 109.500 & \multicolumn{2}{|c|}{292.880} & & & & & \\
\hline & 30 & 3 & 31 & 1 & 1 & 107.800 & \multicolumn{2}{|c|}{276.144} & & & & & \\
\hline & 4 & 5 & 6 & 6 & 1 & 120.000 & \multicolumn{2}{|c|}{585.760} & & & & & \\
\hline & 26 & 1 & 27 & & 1 & 107.800 & \multicolumn{2}{|c|}{276.144} & & & & & \\
\hline & 52 & $5 \overline{1}$ & 56 & & 1 & 120.000 & \multicolumn{2}{|c|}{292.880} & & & & & \\
\hline & 46 & 45 & 60 & & 1 & 120.000 & \multicolumn{2}{|c|}{292.880} & & & & & \\
\hline & 12 & 11 & 16 & 6 & 1 & 120.000 & \multicolumn{2}{|c|}{527.184} & & & & & \\
\hline & 50 & 49 & 54 & & 1 & 120.000 & \multicolumn{2}{|c|}{292.880} & & & & & \\
\hline & 66 & 65 & 70 & & 1 & 117.300 & \multicolumn{2}{|c|}{711.280} & & & & & \\
\hline \multicolumn{14}{|c|}{ [ dihedrals ] } \\
\hline ; & \multicolumn{6}{|c|}{ IMPROPER DIHEDRAL ANGLES } & & & & & & & \\
\hline ; & ai & $a j$ & ak & & al & nct & $\mathbf{C}$ & & $\mathrm{c} 1$ & & $\mathrm{C} 2$ & & c3 3 \\
\hline & 61 & s & 9 & 8 & 10 & 4 & 180.0 & & 10.460 & 2 & & & \\
\hline & 44 & 15 & & 14 & 16 & 4 & 180.0 & & 10.460 & 2 & & & \\
\hline & 16 & 1. & & 7 & 12 & 4 & 180.0 & & 10.460 & 2 & & & \\
\hline & 11 & 7 & 7 & 4 & 8 & 4 & 180.0 & & 10.460 & 2 & & & \\
\hline & 10 & 5 & 5 & 4 & 6 & 4 & 180.0 & & 10.460 & 2 & & & \\
\hline & 7 & 4 & 4 & 3 & 5 & 4 & 180.0 & & 10.460 & 2 & & & \\
\hline & 64 & 65 & & 66 & 70 & 4 & 180.0 & & 10.460 & 2 & & & \\
\hline & 49 & $4 \varepsilon$ & & 53 & 47 & 4 & 180.0 & & 10.460 & 2 & & & \\
\hline & 70 & 61 & & 9 & 62 & 4 & 180.0 & & 10.460 & 2 & & & \\
\hline & 53 & 44 & & 15 & 45 & 4 & 180.0 & & 10.460 & 2 & & & \\
\hline & 65 & $7 C$ & & 61 & 69 & 4 & 180.0 & & 10.460 & 2 & & & \\
\hline & 48 & 53 & & 52 & 44 & 4 & 180.0 & & 10.460 & 2 & & & \\
\hline & 17 & 12 & & 11 & 13 & 4 & 180.0 & & 10.460 & 2 & & & \\
\hline & 20 & 13 & & 12 & 14 & 4 & 180.0 & & 10.460 & 2 & & & \\
\hline & 71 & 62 & & 61 & 63 & 4 & 180.0 & & 10.460 & 2 & & & \\
\hline & 60 & 45 & & 44 & 46 & 4 & 180.0 & & 10.460 & 2 & & & \\
\hline & 35 & 14 & & 13 & 15 & 4 & 180.0 & & 10.460 & 2 & & & \\
\hline & 34 & 10 & & 5 & 9 & 4 & 180.0 & & 10.460 & 2 & & & \\
\hline & 55 & 50 & & 49 & 51 & 4 & 180.0 & & 10.460 & 2 & & & \\
\hline & 72 & 63 & & 62 & 64 & 4 & 180.0 & & 10.460 & 2 & & & \\
\hline & 59 & 46 & & 45 & 47 & 4 & 180.0 & & 10.460 & 2 & & & \\
\hline & 75 & 67 & & 66 & 68 & 4 & 180.0 & & 10.460 & 2 & & & \\
\hline & 76 & $6 \varepsilon$ & & 67 & 69 & 4 & 180.0 & & 10.460 & 2 & & & \\
\hline & 56 & 5. & & 50 & 52 & 4 & 180.0 & & 10.460 & 2 & & & \\
\hline & 58 & 47 & & 46 & 48 & 4 & 180.0 & & 10.460 & 2 & & & \\
\hline & 57 & 52 & & 51 & 53 & 4 & 180.0 & & 10.460 & 2 & & & \\
\hline & 77 & 65 & & 68 & 70 & 4 & 180.0 & & 10.460 & 2 & & & \\
\hline & 73 & 64 & & 63 & 65 & 4 & 180.0 & & 10.460 & 2 & & & \\
\hline & 74 & 66 & & 65 & 67 & 4 & 180.0 & & 10.460 & 2 & & & \\
\hline & 54 & 45 & & 48 & 50 & 4 & 180.0 & & 10.460 & 2 & & & \\
\hline & 22 & 16 & & 11 & 15 & 4 & 180.0 & & 10.460 & 2 & & & \\
\hline & 21 & $\varepsilon$ & 8 & 7 & 9 & 4 & 180.0 & & 10.460 & 2 & & & \\
\hline & dihe & cals & ] & & & & & & & & & & \\
\hline & PROP & $\mathrm{DIF}$ & HEDRAI & & JGLES & & & & & & & & \\
\hline ; & ai & $a j$ & $\begin{array}{l}a k \\
c 5\end{array}$ & & al & net & C & & $\mathrm{C} 1$ & & $\mathrm{C} 2$ & & c3 \\
\hline & 44 & 15 & 16 & 11 & & 3 & 30.334 & 0.000 & -30.334 & -0.000 & -0.000 & 0.000 & \\
\hline & 61 & 9 & 10 & 5 & & 3 & 30.334 & 0.000 & -30.334 & -0.000 & -0.000 & 0.000 & \\
\hline & 44 & 15 & 14 & 13 & & 3 & 30.334 & 0.000 & -30.334 & -0.000 & -0.000 & 0.000 & \\
\hline & 61 & 9 & 8 & 7 & & 3 & 30.334 & 0.000 & -30.334 & -0.000 & -0.000 & 0.000 & \\
\hline & 44 & 15 & 14 & 35 & & 3 & 30.334 & 0.000 & -30.334 & -0.000 & -0.000 & 0.000 & \\
\hline & 61 & 9 & 10 & 34 & & 3 & 30.334 & 0.000 & -30.334 & -0.000 & -0.000 & 0.000 & \\
\hline & 44 & 15 & 16 & 22 & & 3 & 30.334 & 0.000 & -30.334 & -0.000 & -0.000 & 0.000 & \\
\hline & 61 & 9 & 8 & 21 & & 3 & 30.334 & 0.000 & -30.334 & -0.000 & -0.000 & 0.000 & \\
\hline & 9 & 10 & 5 & 4 & & 3 & 30.334 & 0.000 & -30.334 & -0.000 & -0.000 & 0.000 & \\
\hline & 15 & 16 & 11 & 7 & & 3 & 30.334 & 0.000 & -30.334 & -0.000 & -0.000 & 0.000 & \\
\hline & 15 & 16 & $\begin{array}{l}11 \\
11\end{array}$ & 12 & & 3 & 30.334 & 0.000 & -30.334 & -0.000 & -0.000 & 0.000 & \\
\hline & 9 & 8 & $\begin{array}{r}1 \\
7\end{array}$ & $\begin{array}{r}12 \\
4\end{array}$ & & 3 & 30.334 & 0.000 & -30.334 & -0.000 & -0.000 & 0.000 & \\
\hline & 15 & 14 & 13 & 12 & & 3 & 30.334 & 0.000 & -30.334 & -0.000 & -0.000 & 0.000 & \\
\hline
\end{tabular}




\begin{tabular}{|c|c|c|c|c|c|c|c|c|c|c|}
\hline 9 & 10 & 5 & 6 & 3 & 30.334 & 0.000 & -30.334 & -0.000 & -0.000 & 0.000 \\
\hline 62 & 61 & 9 & 8 & 3 & 9.079 & 0.000 & -9.079 & -0.000 & -0.000 & 0.000 \\
\hline 62 & 61 & 9 & 10 & 3 & 9.079 & 0.000 & -9.079 & -0.000 & -0.000 & 0.000 \\
\hline 45 & 44 & 15 & 16 & 3 & 9.079 & 0.000 & -9.079 & -0.000 & -0.000 & 0.000 \\
\hline 45 & 44 & 15 & 14 & 3 & 9.079 & 0.000 & -9.079 & -0.000 & -0.000 & 0.000 \\
\hline 16 & 15 & 14 & 13 & 3 & 30.334 & 0.000 & -30.334 & -0.000 & -0.000 & 0.000 \\
\hline 14 & 15 & 16 & 11 & 3 & 30.334 & 0.000 & -30.334 & -0.000 & -0.000 & 0.000 \\
\hline 10 & 9 & 8 & 7 & 3 & 30.334 & 0.000 & -30.334 & -0.000 & -0.000 & 0.000 \\
\hline 8 & 9 & 10 & 5 & 3 & 30.334 & 0.000 & -30.334 & -0.000 & -0.000 & 0.000 \\
\hline 46 & 45 & 44 & 15 & 3 & 30.334 & 0.000 & -30.334 & -0.000 & -0.000 & 0.000 \\
\hline 63 & 62 & 61 & 9 & 3 & 30.334 & 0.000 & -30.334 & -0.000 & -0.000 & 0.000 \\
\hline 11 & 7 & 8 & 9 & 3 & 30.334 & 0.000 & -30.334 & -0.000 & -0.000 & 0.000 \\
\hline 47 & 46 & 45 & 44 & 3 & 30.334 & 0.000 & -30.334 & -0.000 & -0.000 & 0.000 \\
\hline 64 & 63 & 62 & 61 & 3 & 30.334 & 0.000 & -30.334 & -0.000 & -0.000 & 0.000 \\
\hline 12 & 11 & 7 & 8 & 3 & 30.334 & 0.000 & -30.334 & -0.000 & -0.000 & 0.000 \\
\hline 52 & 51 & 50 & 49 & 3 & 30.334 & 0.000 & -30.334 & -0.000 & -0.000 & 0.000 \\
\hline 16 & 11 & 12 & 13 & 3 & 30.334 & 0.000 & -30.334 & -0.000 & -0.000 & 0.000 \\
\hline 14 & 13 & 12 & 11 & 3 & 30.334 & 0.000 & -30.334 & -0.000 & -0.000 & 0.000 \\
\hline 16 & 11 & 7 & 4 & 3 & 30.334 & 0.000 & -30.334 & -0.000 & -0.000 & 0.000 \\
\hline 10 & 5 & 4 & 7 & 3 & 30.334 & 0.000 & -30.334 & -0.000 & -0.000 & 0.000 \\
\hline 12 & 11 & 7 & 4 & 3 & 30.334 & 0.000 & -30.334 & -0.000 & -0.000 & 0.000 \\
\hline 13 & 12 & 11 & 7 & 3 & 30.334 & 0.000 & -30.334 & -0.000 & -0.000 & 0.000 \\
\hline 8 & 7 & 4 & 5 & 3 & 30.334 & 0.000 & -30.334 & -0.000 & -0.000 & 0.000 \\
\hline 69 & 68 & 67 & 66 & 3 & 30.334 & 0.000 & -30.334 & -0.000 & -0.000 & 0.000 \\
\hline 16 & 11 & 7 & 8 & 3 & 30.334 & 0.000 & -30.334 & -0.000 & -0.000 & 0.000 \\
\hline 11 & 7 & 4 & 5 & 3 & 30.334 & 0.000 & -30.334 & -0.000 & -0.000 & 0.000 \\
\hline 68 & 67 & 66 & 65 & 3 & 30.334 & 0.000 & -30.334 & -0.000 & -0.000 & 0.000 \\
\hline 51 & 50 & 49 & 48 & 3 & 30.334 & 0.000 & -30.334 & -0.000 & -0.000 & 0.000 \\
\hline 7 & 4 & 5 & 6 & 3 & 30.334 & 0.000 & -30.334 & -0.000 & -0.000 & 0.000 \\
\hline 10 & 5 & 4 & 3 & 3 & 30.334 & 0.000 & -30.334 & -0.000 & -0.000 & 0.000 \\
\hline 11 & 7 & 4 & 3 & 3 & 30.334 & 0.000 & -30.334 & -0.000 & -0.000 & 0.000 \\
\hline 8 & 7 & 4 & 3 & 3 & 30.334 & 0.000 & -30.334 & -0.000 & -0.000 & 0.000 \\
\hline 51 & 52 & 53 & 44 & 3 & 29.288 & 0.000 & -29.288 & -0.000 & -0.000 & 0.000 \\
\hline 68 & 69 & 70 & 61 & 3 & 29.288 & 0.000 & -29.288 & -0.000 & -0.000 & 0.000 \\
\hline 67 & 66 & 65 & 64 & 3 & 29.288 & 0.000 & -29.288 & -0.000 & -0.000 & 0.000 \\
\hline 50 & 49 & 48 & 47 & 3 & 29.288 & 0.000 & -29.288 & -0.000 & -0.000 & 0.000 \\
\hline 51 & 52 & 53 & 48 & 3 & 29.288 & 0.000 & -29.288 & -0.000 & -0.000 & 0.000 \\
\hline 68 & 69 & 70 & 65 & 3 & 29.288 & 0.000 & -29.288 & -0.000 & -0.000 & 0.000 \\
\hline 7 & 4 & 3 & 2 & 3 & 0.000 & 0.000 & 0.000 & -0.000 & -0.000 & 0.000 \\
\hline 5 & 4 & 3 & 2 & 3 & 0.000 & 0.000 & 0.000 & -0.000 & -0.000 & 0.000 \\
\hline 4 & 5 & 6 & 1 & 3 & 0.000 & 0.000 & 0.000 & -0.000 & -0.000 & 0.000 \\
\hline 10 & 5 & 6 & 1 & 3 & 0.000 & 0.000 & 0.000 & -0.000 & -0.000 & 0.000 \\
\hline 52 & 53 & 44 & 15 & 3 & 30.334 & 0.000 & -30.334 & -0.000 & -0.000 & 0.000 \\
\hline 69 & 70 & 61 & 9 & 3 & 30.334 & 0.000 & -30.334 & -0.000 & -0.000 & 0.000 \\
\hline 69 & 70 & 61 & 62 & 3 & 30.334 & 0.000 & -30.334 & -0.000 & -0.000 & 0.000 \\
\hline 52 & 53 & 44 & 45 & 3 & 30.334 & 0.000 & -30.334 & -0.000 & -0.000 & 0.000 \\
\hline 66 & 65 & 64 & 63 & 3 & 29.288 & 0.000 & -29.288 & -0.000 & -0.000 & 0.000 \\
\hline 49 & 48 & 47 & 46 & 3 & 29.288 & 0.000 & -29.288 & -0.000 & -0.000 & 0.000 \\
\hline 47 & 48 & 53 & 44 & 3 & 30.334 & 0.000 & -30.334 & -0.000 & -0.000 & 0.000 \\
\hline 49 & 48 & 53 & 44 & 3 & 30.334 & 0.000 & -30.334 & -0.000 & -0.000 & 0.000 \\
\hline 64 & 65 & 70 & 61 & 3 & 30.334 & 0.000 & -30.334 & -0.000 & -0.000 & 0.000 \\
\hline 66 & 65 & 70 & 61 & 3 & 30.334 & 0.000 & -30.334 & -0.000 & -0.000 & 0.000 \\
\hline 69 & 70 & 65 & 66 & 3 & 30.334 & 0.000 & -30.334 & -0.000 & -0.000 & 0.000 \\
\hline 69 & 70 & 65 & 64 & 3 & 30.334 & 0.000 & -30.334 & -0.000 & -0.000 & 0.000 \\
\hline 52 & 53 & 48 & 49 & 3 & 30.334 & 0.000 & -30.334 & -0.000 & -0.000 & 0.000 \\
\hline 52 & 53 & 48 & 47 & 3 & 30.334 & 0.000 & -30.334 & -0.000 & -0.000 & 0.000 \\
\hline 4 & 3 & 2 & 1 & 3 & 2.301 & -1.464 & 0.837 & -1.674 & -0.000 & 0.000 \\
\hline 5 & 6 & 1 & 2 & 3 & 2.301 & -1.464 & 0.837 & -1.674 & -0.000 & 0.000 \\
\hline 70 & 61 & 9 & 8 & 3 & 9.079 & 0.000 & -9.079 & -0.000 & -0.000 & 0.000 \\
\hline 70 & 61 & 9 & 10 & 3 & 9.079 & 0.000 & -9.079 & -0.000 & -0.000 & 0.000 \\
\hline 53 & 44 & 15 & 14 & 3 & 9.079 & 0.000 & -9.079 & -0.000 & -0.000 & 0.000 \\
\hline 53 & 44 & 15 & 16 & 3 & 9.079 & 0.000 & -9.079 & -0.000 & -0.000 & 0.000 \\
\hline 70 & 61 & 62 & 63 & 3 & 30.334 & 0.000 & -30.334 & -0.000 & -0.000 & 0.000 \\
\hline 53 & 44 & 45 & 46 & 3 & 30.334 & 0.000 & -30.334 & -0.000 & -0.000 & 0.000 \\
\hline 65 & 64 & 63 & 62 & 3 & 30.334 & 0.000 & -30.334 & -0.000 & -0.000 & 0.000 \\
\hline
\end{tabular}




\begin{tabular}{|c|c|c|c|c|c|c|c|c|c|c|}
\hline 48 & 47 & 46 & 45 & 3 & 30.334 & 0.000 & -30.334 & -0.000 & -0.000 & 0.000 \\
\hline 53 & 52 & 51 & 50 & 3 & 30.334 & 0.000 & -30.334 & -0.000 & -0.000 & 0.000 \\
\hline 70 & 69 & 68 & 67 & 3 & 30.334 & 0.000 & -30.334 & -0.000 & -0.000 & 0.000 \\
\hline 65 & 70 & 61 & 9 & 3 & 30.334 & 0.000 & -30.334 & -0.000 & -0.000 & 0.000 \\
\hline 48 & 53 & 44 & 15 & 3 & 30.334 & 0.000 & -30.334 & -0.000 & -0.000 & 0.000 \\
\hline 65 & 70 & 61 & 62 & 3 & 9.079 & 0.000 & -9.079 & -0.000 & -0.000 & 0.000 \\
\hline 48 & 53 & 44 & 45 & 3 & 9.079 & 0.000 & -9.079 & -0.000 & -0.000 & 0.000 \\
\hline 53 & 48 & 49 & 50 & 3 & 29.288 & 0.000 & -29.288 & -0.000 & -0.000 & 0.000 \\
\hline 70 & 65 & 64 & 63 & 3 & 29.288 & 0.000 & -29.288 & -0.000 & -0.000 & 0.000 \\
\hline 53 & 48 & 47 & 46 & 3 & 29.288 & 0.000 & -29.288 & -0.000 & -0.000 & 0.000 \\
\hline 70 & 65 & 66 & 67 & 3 & 29.288 & 0.000 & -29.288 & -0.000 & -0.000 & 0.000 \\
\hline 20 & 13 & 14 & 15 & 3 & 30.334 & 0.000 & -30.334 & -0.000 & -0.000 & 0.000 \\
\hline 17 & 12 & 13 & 14 & 3 & 30.334 & 0.000 & -30.334 & -0.000 & -0.000 & 0.000 \\
\hline 17 & 12 & 11 & 7 & 3 & 30.334 & 0.000 & -30.334 & -0.000 & -0.000 & 0.000 \\
\hline 20 & 13 & 12 & 11 & 3 & 30.334 & 0.000 & -30.334 & -0.000 & -0.000 & 0.000 \\
\hline 17 & 12 & 11 & 16 & 3 & 30.334 & 0.000 & -30.334 & -0.000 & -0.000 & 0.000 \\
\hline 20 & 13 & 12 & 17 & 3 & 30.334 & 0.000 & -30.334 & -0.000 & -0.000 & 0.000 \\
\hline 6 & 5 & 4 & 3 & 3 & 30.334 & 0.000 & -30.334 & -0.000 & -0.000 & 0.000 \\
\hline 19 & 20 & 13 & 12 & 3 & 0.000 & 0.000 & 0.000 & -0.000 & -0.000 & 0.000 \\
\hline 19 & 20 & 13 & 14 & 3 & 0.000 & 0.000 & 0.000 & -0.000 & -0.000 & 0.000 \\
\hline 18 & 17 & 12 & 13 & 3 & 0.000 & 0.000 & 0.000 & -0.000 & -0.000 & 0.000 \\
\hline 18 & 17 & 12 & 11 & 3 & 0.000 & 0.000 & 0.000 & -0.000 & -0.000 & 0.000 \\
\hline 19 & 18 & 17 & 12 & 3 & 2.301 & -1.464 & 0.837 & -1.674 & -0.000 & 0.000 \\
\hline 18 & 19 & 20 & 13 & 3 & 2.301 & -1.464 & 0.837 & -1.674 & -0.000 & 0.000 \\
\hline 20 & 19 & 18 & 17 & 3 & 2.301 & -1.464 & 0.837 & -1.674 & -0.000 & 0.000 \\
\hline 6 & 1 & 2 & 3 & 3 & 2.301 & -1.464 & 0.837 & -1.674 & -0.000 & 0.000 \\
\hline 60 & 45 & 44 & 15 & 3 & 30.334 & 0.000 & -30.334 & -0.000 & -0.000 & 0.000 \\
\hline 71 & 62 & 61 & 9 & 3 & 30.334 & 0.000 & -30.334 & -0.000 & -0.000 & 0.000 \\
\hline 34 & 10 & 9 & 8 & 3 & 30.334 & 0.000 & -30.334 & -0.000 & -0.000 & 0.000 \\
\hline 35 & 14 & 15 & 16 & 3 & 30.334 & 0.000 & -30.334 & -0.000 & -0.000 & 0.000 \\
\hline 60 & 45 & 44 & 53 & 3 & 30.334 & 0.000 & -30.334 & -0.000 & -0.000 & 0.000 \\
\hline 71 & 62 & 61 & 70 & 3 & 30.334 & 0.000 & -30.334 & -0.000 & -0.000 & 0.000 \\
\hline 72 & 63 & 62 & 61 & 3 & 30.334 & 0.000 & -30.334 & -0.000 & -0.000 & 0.000 \\
\hline 59 & 46 & 45 & 44 & 3 & 30.334 & 0.000 & -30.334 & -0.000 & -0.000 & 0.000 \\
\hline 57 & 52 & 51 & 50 & 3 & 30.334 & 0.000 & -30.334 & -0.000 & -0.000 & 0.000 \\
\hline 77 & 69 & 68 & 67 & 3 & 30.334 & 0.000 & -30.334 & -0.000 & -0.000 & 0.000 \\
\hline 35 & 14 & 13 & 12 & 3 & 30.334 & 0.000 & -30.334 & -0.000 & -0.000 & 0.000 \\
\hline 55 & 50 & 51 & 52 & 3 & 30.334 & 0.000 & -30.334 & -0.000 & -0.000 & 0.000 \\
\hline 71 & 62 & 63 & 64 & 3 & 30.334 & 0.000 & -30.334 & -0.000 & -0.000 & 0.000 \\
\hline 73 & 64 & 63 & 62 & 3 & 30.334 & 0.000 & -30.334 & -0.000 & -0.000 & 0.000 \\
\hline 75 & 67 & 68 & 69 & 3 & 30.334 & 0.000 & -30.334 & -0.000 & -0.000 & 0.000 \\
\hline 74 & 66 & 67 & 68 & 3 & 30.334 & 0.000 & -30.334 & -0.000 & -0.000 & 0.000 \\
\hline 56 & 51 & 50 & 49 & 3 & 30.334 & 0.000 & -30.334 & -0.000 & -0.000 & 0.000 \\
\hline 54 & 49 & 50 & 51 & 3 & 30.334 & 0.000 & -30.334 & -0.000 & -0.000 & 0.000 \\
\hline 76 & 68 & 67 & 66 & 3 & 30.334 & 0.000 & -30.334 & -0.000 & -0.000 & 0.000 \\
\hline 34 & 10 & 5 & 4 & 3 & 30.334 & 0.000 & -30.334 & -0.000 & -0.000 & 0.000 \\
\hline 58 & 47 & 46 & 45 & 3 & 30.334 & 0.000 & -30.334 & -0.000 & -0.000 & 0.000 \\
\hline 60 & 45 & 46 & 47 & 3 & 30.334 & 0.000 & -30.334 & -0.000 & -0.000 & 0.000 \\
\hline 72 & 63 & 64 & 65 & 3 & 30.334 & 0.000 & -30.334 & -0.000 & -0.000 & 0.000 \\
\hline 59 & 46 & 47 & 48 & 3 & 30.334 & 0.000 & -30.334 & -0.000 & -0.000 & 0.000 \\
\hline 76 & 68 & 69 & 70 & 3 & 30.334 & 0.000 & -30.334 & -0.000 & -0.000 & 0.000 \\
\hline 75 & 67 & 66 & 65 & 3 & 30.334 & 0.000 & -30.334 & -0.000 & -0.000 & 0.000 \\
\hline 56 & 51 & 52 & 53 & 3 & 30.334 & 0.000 & -30.334 & -0.000 & -0.000 & 0.000 \\
\hline 55 & 50 & 49 & 48 & 3 & 30.334 & 0.000 & -30.334 & -0.000 & -0.000 & 0.000 \\
\hline 34 & 10 & 5 & 6 & 3 & 30.334 & 0.000 & -30.334 & -0.000 & -0.000 & 0.000 \\
\hline 35 & 14 & 13 & 20 & 3 & 30.334 & 0.000 & -30.334 & -0.000 & -0.000 & 0.000 \\
\hline 55 & 50 & 49 & 54 & 3 & 30.334 & 0.000 & -30.334 & -0.000 & -0.000 & 0.000 \\
\hline 76 & 68 & 67 & 75 & 3 & 30.334 & 0.000 & -30.334 & -0.000 & -0.000 & 0.000 \\
\hline 73 & 64 & 63 & 72 & 3 & 30.334 & 0.000 & -30.334 & -0.000 & -0.000 & 0.000 \\
\hline 60 & 45 & 46 & 59 & 3 & 30.334 & 0.000 & -30.334 & -0.000 & -0.000 & 0.000 \\
\hline 56 & 51 & 50 & 55 & 3 & 30.334 & 0.000 & -30.334 & -0.000 & -0.000 & 0.000 \\
\hline 75 & 67 & 66 & 74 & 3 & 30.334 & 0.000 & -30.334 & -0.000 & -0.000 & 0.000 \\
\hline 59 & 46 & 47 & 58 & 3 & 30.334 & 0.000 & -30.334 & -0.000 & -0.000 & 0.000 \\
\hline 72 & 63 & 62 & 71 & 3 & 30.334 & 0.000 & -30.334 & -0.000 & -0.000 & 0.000 \\
\hline 77 & 69 & 68 & 76 & 3 & 30.334 & 0.000 & -30.334 & -0.000 & -0.000 & 0.000 \\
\hline
\end{tabular}




\begin{tabular}{|c|c|c|c|c|c|c|c|c|c|c|}
\hline 57 & 52 & 51 & 56 & 3 & 30.334 & 0.000 & -30.334 & -0.000 & -0.000 & 0.000 \\
\hline 57 & 52 & 53 & 44 & 3 & 30.334 & 0.000 & -30.334 & -0.000 & -0.000 & 0.000 \\
\hline 77 & 69 & 70 & 61 & 3 & 30.334 & 0.000 & -30.334 & -0.000 & -0.000 & 0.000 \\
\hline 58 & 47 & 48 & 49 & 3 & 30.334 & 0.000 & -30.334 & -0.000 & -0.000 & 0.000 \\
\hline 54 & 49 & 48 & 47 & 3 & 30.334 & 0.000 & -30.334 & -0.000 & -0.000 & 0.000 \\
\hline 74 & 66 & 65 & 64 & 3 & 30.334 & 0.000 & -30.334 & -0.000 & -0.000 & 0.000 \\
\hline 73 & 64 & 65 & 66 & 3 & 30.334 & 0.000 & -30.334 & -0.000 & -0.000 & 0.000 \\
\hline 74 & 66 & 65 & 70 & 3 & 30.334 & 0.000 & -30.334 & -0.000 & -0.000 & 0.000 \\
\hline 73 & 64 & 65 & 70 & 3 & 30.334 & 0.000 & -30.334 & -0.000 & -0.000 & 0.000 \\
\hline 57 & 52 & 53 & 48 & 3 & 30.334 & 0.000 & -30.334 & -0.000 & -0.000 & 0.000 \\
\hline 77 & 69 & 70 & 65 & 3 & 30.334 & 0.000 & -30.334 & -0.000 & -0.000 & 0.000 \\
\hline 54 & 49 & 48 & 53 & 3 & 30.334 & 0.000 & -30.334 & -0.000 & -0.000 & 0.000 \\
\hline 58 & 47 & 48 & 53 & 3 & 30.334 & 0.000 & -30.334 & -0.000 & -0.000 & 0.000 \\
\hline 43 & 20 & 13 & 14 & 3 & 0.000 & 0.000 & 0.000 & -0.000 & -0.000 & 0.000 \\
\hline 33 & 6 & 5 & 4 & 3 & 0.000 & 0.000 & 0.000 & -0.000 & -0.000 & 0.000 \\
\hline 43 & 20 & 13 & 12 & 3 & 0.000 & 0.000 & 0.000 & -0.000 & -0.000 & 0.000 \\
\hline 36 & 17 & 12 & 13 & 3 & 0.000 & 0.000 & 0.000 & -0.000 & -0.000 & 0.000 \\
\hline 42 & 20 & 13 & 12 & 3 & 0.000 & 0.000 & 0.000 & -0.000 & -0.000 & 0.000 \\
\hline 32 & 6 & 5 & 4 & 3 & 0.000 & 0.000 & 0.000 & -0.000 & -0.000 & 0.000 \\
\hline 33 & 6 & 5 & 10 & 3 & 0.000 & 0.000 & 0.000 & -0.000 & -0.000 & 0.000 \\
\hline 30 & 3 & 4 & 5 & 3 & 0.000 & 0.000 & 0.000 & -0.000 & -0.000 & 0.000 \\
\hline 30 & 3 & 4 & 7 & 3 & 0.000 & 0.000 & 0.000 & -0.000 & -0.000 & 0.000 \\
\hline 42 & 20 & 13 & 14 & 3 & 0.000 & 0.000 & 0.000 & -0.000 & -0.000 & 0.000 \\
\hline 31 & 3 & 4 & 5 & 3 & 0.000 & 0.000 & 0.000 & -0.000 & -0.000 & 0.000 \\
\hline 32 & 6 & 5 & 10 & 3 & 0.000 & 0.000 & 0.000 & -0.000 & -0.000 & 0.000 \\
\hline 37 & 17 & 12 & 11 & 3 & 0.000 & 0.000 & 0.000 & -0.000 & -0.000 & 0.000 \\
\hline 31 & 3 & 4 & 7 & 3 & 0.000 & 0.000 & 0.000 & -0.000 & -0.000 & 0.000 \\
\hline 36 & 17 & 12 & 11 & 3 & 0.000 & 0.000 & 0.000 & -0.000 & -0.000 & 0.000 \\
\hline 37 & 17 & 12 & 13 & 3 & 0.000 & 0.000 & 0.000 & -0.000 & -0.000 & 0.000 \\
\hline 41 & 19 & 20 & 13 & 3 & 0.967 & 2.900 & 0.000 & -3.866 & -0.000 & 0.000 \\
\hline 26 & 1 & 6 & 5 & 3 & 0.967 & 2.900 & 0.000 & -3.866 & -0.000 & 0.000 \\
\hline 38 & 18 & 17 & 12 & 3 & 0.967 & 2.900 & 0.000 & -3.866 & -0.000 & 0.000 \\
\hline 39 & 18 & 17 & 12 & 3 & 0.967 & 2.900 & 0.000 & -3.866 & -0.000 & 0.000 \\
\hline 28 & 2 & 3 & 4 & 3 & 0.967 & 2.900 & 0.000 & -3.866 & -0.000 & 0.000 \\
\hline 40 & 19 & 20 & 13 & 3 & 0.967 & 2.900 & 0.000 & -3.866 & -0.000 & 0.000 \\
\hline 27 & 1 & 6 & 5 & 3 & 0.967 & 2.900 & 0.000 & -3.866 & -0.000 & 0.000 \\
\hline 29 & 2 & 3 & 4 & 3 & 0.967 & 2.900 & 0.000 & -3.866 & -0.000 & 0.000 \\
\hline 27 & 1 & 2 & 3 & 3 & 0.628 & 1.883 & 0.000 & -2.510 & -0.000 & 0.000 \\
\hline 29 & 2 & 1 & 6 & 3 & 0.628 & 1.883 & 0.000 & -2.510 & -0.000 & 0.000 \\
\hline 32 & 6 & 1 & 2 & 3 & 0.628 & 1.883 & 0.000 & -2.510 & -0.000 & 0.000 \\
\hline 36 & 17 & 18 & 19 & 3 & 0.628 & 1.883 & 0.000 & -2.510 & -0.000 & 0.000 \\
\hline 38 & 18 & 19 & 20 & 3 & 0.628 & 1.883 & 0.000 & -2.510 & -0.000 & 0.000 \\
\hline 39 & 18 & 19 & 20 & 3 & 0.628 & 1.883 & 0.000 & -2.510 & -0.000 & 0.000 \\
\hline 40 & 19 & 18 & 17 & 3 & 0.628 & 1.883 & 0.000 & -2.510 & -0.000 & 0.000 \\
\hline 42 & 20 & 19 & 18 & 3 & 0.628 & 1.883 & 0.000 & -2.510 & -0.000 & 0.000 \\
\hline 41 & 19 & 18 & 17 & 3 & 0.628 & 1.883 & 0.000 & -2.510 & -0.000 & 0.000 \\
\hline 26 & 1 & 2 & 3 & 3 & 0.628 & 1.883 & 0.000 & -2.510 & -0.000 & 0.000 \\
\hline 30 & 3 & 2 & 1 & 3 & 0.628 & 1.883 & 0.000 & -2.510 & -0.000 & 0.000 \\
\hline 43 & 20 & 19 & 18 & 3 & 0.628 & 1.883 & 0.000 & -2.510 & -0.000 & 0.000 \\
\hline 28 & 2 & 1 & 6 & 3 & 0.628 & 1.883 & 0.000 & -2.510 & -0.000 & 0.000 \\
\hline 31 & 3 & 2 & 1 & 3 & 0.628 & 1.883 & 0.000 & -2.510 & -0.000 & 0.000 \\
\hline 33 & 6 & 1 & 2 & 3 & 0.628 & 1.883 & 0.000 & -2.510 & -0.000 & 0.000 \\
\hline 37 & 17 & 18 & 19 & 3 & 0.628 & 1.883 & 0.000 & -2.510 & -0.000 & 0.000 \\
\hline 39 & 18 & 17 & 37 & 3 & 0.628 & 1.883 & 0.000 & -2.510 & -0.000 & 0.000 \\
\hline 38 & 18 & 17 & 37 & 3 & 0.628 & 1.883 & 0.000 & -2.510 & -0.000 & 0.000 \\
\hline 40 & 19 & 18 & 38 & 3 & 0.628 & 1.883 & 0.000 & -2.510 & -0.000 & 0.000 \\
\hline 43 & 20 & 19 & 41 & 3 & 0.628 & 1.883 & 0.000 & -2.510 & -0.000 & 0.000 \\
\hline 31 & 3 & 2 & 29 & 3 & 0.628 & 1.883 & 0.000 & -2.510 & -0.000 & 0.000 \\
\hline 41 & 19 & 18 & 39 & 3 & 0.628 & 1.883 & 0.000 & -2.510 & -0.000 & 0.000 \\
\hline 28 & 2 & 1 & 27 & 3 & 0.628 & 1.883 & 0.000 & -2.510 & -0.000 & 0.000 \\
\hline 42 & 20 & 19 & 40 & 3 & 0.628 & 1.883 & 0.000 & -2.510 & -0.000 & 0.000 \\
\hline 43 & 20 & 19 & 40 & 3 & 0.628 & 1.883 & 0.000 & -2.510 & -0.000 & 0.000 \\
\hline 42 & 20 & 19 & 41 & 3 & 0.628 & 1.883 & 0.000 & -2.510 & -0.000 & 0.000 \\
\hline 30 & 3 & 2 & 29 & 3 & 0.628 & 1.883 & 0.000 & -2.510 & -0.000 & 0.000 \\
\hline 31 & 3 & 2 & 28 & 3 & 0.628 & 1.883 & 0.000 & -2.510 & -0.000 & 0.000 \\
\hline
\end{tabular}




$\begin{array}{rrrrrrrrrrr}38 & 18 & 17 & 36 & 3 & 0.628 & 1.883 & 0.000 & -2.510 & -0.000 & 0.000 \\ 30 & 3 & 2 & 28 & 3 & 0.628 & 1.883 & 0.000 & -2.510 & -0.000 & 0.000 \\ 33 & 6 & 1 & 27 & 3 & 0.628 & 1.883 & 0.000 & -2.510 & -0.000 & 0.000 \\ 29 & 2 & 1 & 27 & 3 & 0.628 & 1.883 & 0.000 & -2.510 & -0.000 & 0.000 \\ 29 & 2 & 1 & 26 & 3 & 0.628 & 1.883 & 0.000 & -2.510 & -0.000 & 0.000 \\ 32 & 6 & 1 & 27 & 3 & 0.628 & 1.883 & 0.000 & -2.510 & -0.000 & 0.000 \\ 32 & 6 & 1 & 26 & 3 & 0.628 & 1.883 & 0.000 & -2.510 & -0.000 & 0.000 \\ 39 & 18 & 17 & 36 & 3 & 0.628 & 1.883 & 0.000 & -2.510 & -0.000 & 0.000 \\ 28 & 2 & 1 & 26 & 3 & 0.628 & 1.883 & 0.000 & -2.510 & -0.000 & 0.000 \\ 33 & 6 & 1 & 26 & 3 & 0.628 & 1.883 & 0.000 & -2.510 & -0.000 & 0.000 \\ 41 & 19 & 18 & 38 & 3 & 0.628 & 1.883 & 0.000 & -2.510 & -0.000 & 0.000 \\ 40 & 19 & 18 & 39 & 3 & 0.628 & 1.883 & 0.000 & -2.510 & -0.000 & 0.000 \\ 21 & 8 & 9 & 10 & 3 & 9.079 & 0.000 & -9.079 & -0.000 & -0.000 & 0.000 \\ 22 & 16 & 15 & 14 & 3 & 9.079 & 0.000 & -9.079 & -0.000 & -0.000 & 0.000 \\ 22 & 16 & 11 & 7 & 3 & 30.334 & 0.000 & -30.334 & -0.000 & -0.000 & 0.000 \\ 21 & 8 & 7 & 4 & 3 & 30.334 & 0.000 & -30.334 & -0.000 & -0.000 & 0.000 \\ 22 & 16 & 11 & 12 & 3 & 30.334 & 0.000 & -30.334 & -0.000 & -0.000 & 0.000 \\ 21 & 8 & 7 & 11 & 3 & 30.334 & 0.000 & -30.334 & -0.000 & -0.000 & 0.000 \\ 21 & 23 & 22 & 16 & 3 & 0.000 & 0.000 & 0.000 & -0.000 & -0.000 & 0.000 \\ 22 & 23 & 21 & 8 & 3 & 0.000 & 0.000 & 0.000 & -0.000 & -0.000 & 0.000 \\ 23 & 22 & 16 & 15 & 3 & 12.510 & 0.000 & -12.510 & -0.000 & -0.000 & 0.000 \\ 23 & 21 & 8 & 9 & 3 & 12.510 & 0.000 & -12.510 & -0.000 & -0.000 & 0.000 \\ 23 & 21 & 8 & 7 & 3 & 12.510 & 0.000 & -12.510 & -0.000 & -0.000 & 0.000 \\ 23 & 22 & 16 & 11 & 3 & 12.510 & 0.000 & -12.510 & -0.000 & -0.000 & 0.000 \\ 24 & 23 & 21 & 8 & 3 & 0.000 & 0.000 & 0.000 & -0.000 & -0.000 & 0.000 \\ 25 & 23 & 22 & 16 & 3 & 0.000 & 0.000 & 0.000 & -0.000 & -0.000 & 0.000 \\ 24 & 23 & 22 & 16 & 3 & 0.000 & 0.000 & 0.000 & -0.000 & -0.000 & 0.000 \\ 25 & 23 & 21 & 8 & 3 & 0.000 & 0.000 & 0.000 & -0.000 & -0.000 & 0.000\end{array}$

[ pairs ]

$\begin{array}{rrr}\text { airs } & & \\ 1 & 4 & 1 \\ 2 & 5 & 1 \\ 3 & 6 & 1 \\ 2 & 7 & 1 \\ 3 & 8 & 1 \\ 1 & 10 & 1 \\ 6 & 7 & 1 \\ 5 & 8 & 1 \\ 4 & 9 & 1 \\ 3 & 10 & 1 \\ 3 & 11 & 1 \\ 6 & 9 & 1 \\ 5 & 11 & 1 \\ 4 & 12 & 1 \\ 7 & 10 & 1 \\ 9 & 11 & 1 \\ 8 & 12 & 1 \\ 7 & 13 & 1 \\ 4 & 16 & 1 \\ 7 & 15 & 1 \\ 8 & 16 & 1 \\ 7 & 17 & 1 \\ 11 & 14 & 1 \\ 4 & 21 & 1 \\ 12 & 15 & 1 \\ 13 & 16 & 1 \\ 11 & 18 & 1 \\ 7 & 22 & 1 \\ 3 & 26 & 1 \\ 8 & 22 & 1 \\ 7 & 23 & 1 \\ 3 & 27 & 1 \\ 14 & 17 & 1 \\ 13 & 18 & 1 \\ 12 & 19 & 1 \\ & & \\ 13\end{array}$




\begin{tabular}{|c|c|}
\hline 11 & 20 \\
\hline 10 & 21 \\
\hline 5 & 26 \\
\hline 1 & 30 \\
\hline 11 & 21 \\
\hline 9 & 23 \\
\hline 8 & 24 \\
\hline 5 & 27 \\
\hline 4 & 28 \\
\hline 1 & 31 \\
\hline 16 & 17 \\
\hline 14 & 19 \\
\hline 8 & 25 \\
\hline 4 & 29 \\
\hline 12 & 22 \\
\hline 11 & 23 \\
\hline 6 & 28 \\
\hline 2 & 32 \\
\hline 15 & 20 \\
\hline 6 & 29 \\
\hline 5 & 30 \\
\hline 2 & 33 \\
\hline 14 & 22 \\
\hline 5 & 31 \\
\hline 4 & 32 \\
\hline 17 & 20 \\
\hline 16 & 21 \\
\hline 7 & 30 \\
\hline 4 & 33 \\
\hline 15 & 23 \\
\hline 7 & 31 \\
\hline 4 & 34 \\
\hline 16 & 24 \\
\hline 6 & 34 \\
\hline 16 & 25 \\
\hline 10 & 32 \\
\hline 8 & 34 \\
\hline 10 & 33 \\
\hline 12 & 35 \\
\hline 11 & 36 \\
\hline 11 & 37 \\
\hline 13 & 36 \\
\hline 13 & 37 \\
\hline 12 & 38 \\
\hline 16 & 35 \\
\hline 12 & 39 \\
\hline 13 & 40 \\
\hline 26 & 28 \\
\hline 13 & 41 \\
\hline 12 & 42 \\
\hline 27 & 28 \\
\hline 26 & 29 \\
\hline 20 & 35 \\
\hline 19 & 36 \\
\hline 12 & 43 \\
\hline 11 & 44 \\
\hline 27 & 29 \\
\hline 19 & 37 \\
\hline 14 & 42 \\
\hline 17 & 40 \\
\hline 14 & 43 \\
\hline 13 & 44 \\
\hline 28 & 30 \\
\hline 26 & 32 \\
\hline 20 & 38 \\
\hline
\end{tabular}




\begin{tabular}{|c|c|}
\hline 17 & 41 \\
\hline 29 & 30 \\
\hline 28 & 31 \\
\hline 27 & 32 \\
\hline 26 & 33 \\
\hline 20 & 39 \\
\hline 14 & 45 \\
\hline 29 & 31 \\
\hline 27 & 33 \\
\hline 18 & 42 \\
\hline 18 & 43 \\
\hline 16 & 45 \\
\hline 15 & 46 \\
\hline 15 & 48 \\
\hline 22 & 44 \\
\hline 5 & 61 \\
\hline 15 & 52 \\
\hline 14 & 53 \\
\hline 7 & 61 \\
\hline 16 & 53 \\
\hline 8 & 62 \\
\hline 10 & 62 \\
\hline 9 & 63 \\
\hline 36 & 38 \\
\hline 9 & 65 \\
\hline 37 & 38 \\
\hline 36 & 39 \\
\hline 15 & 60 \\
\hline 37 & 39 \\
\hline 38 & 40 \\
\hline 9 & 69 \\
\hline 8 & 70 \\
\hline 39 & 40 \\
\hline 38 & 41 \\
\hline 35 & 44 \\
\hline 39 & 41 \\
\hline 10 & 70 \\
\hline 9 & 71 \\
\hline 40 & 42 \\
\hline 21 & 61 \\
\hline 41 & 42 \\
\hline 40 & 43 \\
\hline 41 & 43 \\
\hline 44 & 47 \\
\hline 45 & 48 \\
\hline 44 & 49 \\
\hline 46 & 49 \\
\hline 44 & 51 \\
\hline 34 & 61 \\
\hline 47 & 50 \\
\hline 45 & 52 \\
\hline 48 & 51 \\
\hline 47 & 52 \\
\hline 46 & 53 \\
\hline 49 & 52 \\
\hline 47 & 54 \\
\hline 44 & 57 \\
\hline 50 & 53 \\
\hline 48 & 55 \\
\hline 45 & 58 \\
\hline 44 & 59 \\
\hline 51 & 54 \\
\hline 49 & 56 \\
\hline 48 & 57 \\
\hline 53 & 54 \\
\hline
\end{tabular}




$\begin{array}{lll}52 & 55 & 1 \\ 50 & 57 & 1 \\ 49 & 58 & 1 \\ 48 & 59 & 1 \\ 47 & 60 & 1 \\ 54 & 55 & 1 \\ 53 & 56 & 1 \\ 55 & 56 & 1 \\ 53 & 58 & 1 \\ 56 & 57 & 1 \\ 53 & 60 & 1 \\ 58 & 59 & 1 \\ 59 & 60 & 1 \\ 61 & 64 & 1 \\ 62 & 65 & 1 \\ 61 & 66 & 1 \\ 63 & 66 & 1 \\ 61 & 68 & 1 \\ 64 & 67 & 1 \\ 62 & 69 & 1 \\ 65 & 68 & 1 \\ 64 & 69 & 1 \\ 63 & 70 & 1 \\ 61 & 72 & 1 \\ 66 & 69 & 1 \\ 64 & 71 & 1 \\ 62 & 73 & 1 \\ 67 & 70 & 1 \\ 65 & 72 & 1 \\ 64 & 74 & 1 \\ 61 & 77 & 1 \\ 66 & 73 & 1 \\ 65 & 75 & 1 \\ 70 & 71 & 1 \\ 68 & 74 & 1 \\ 66 & 76 & 1 \\ 65 & 77 & 1 \\ 71 & 72 & 1 \\ 70 & 73 & 1 \\ 70 & 74 & 1 \\ 69 & 75 & 1 \\ 67 & 77 & 1 \\ 72 & 73 & 1 \\ 70 & 76 & 1 \\ 74 & 75 & 1 \\ 75 & 76 & 1 \\ 76 & 77 & 1 \\ & & \end{array}$




\section{Cation}

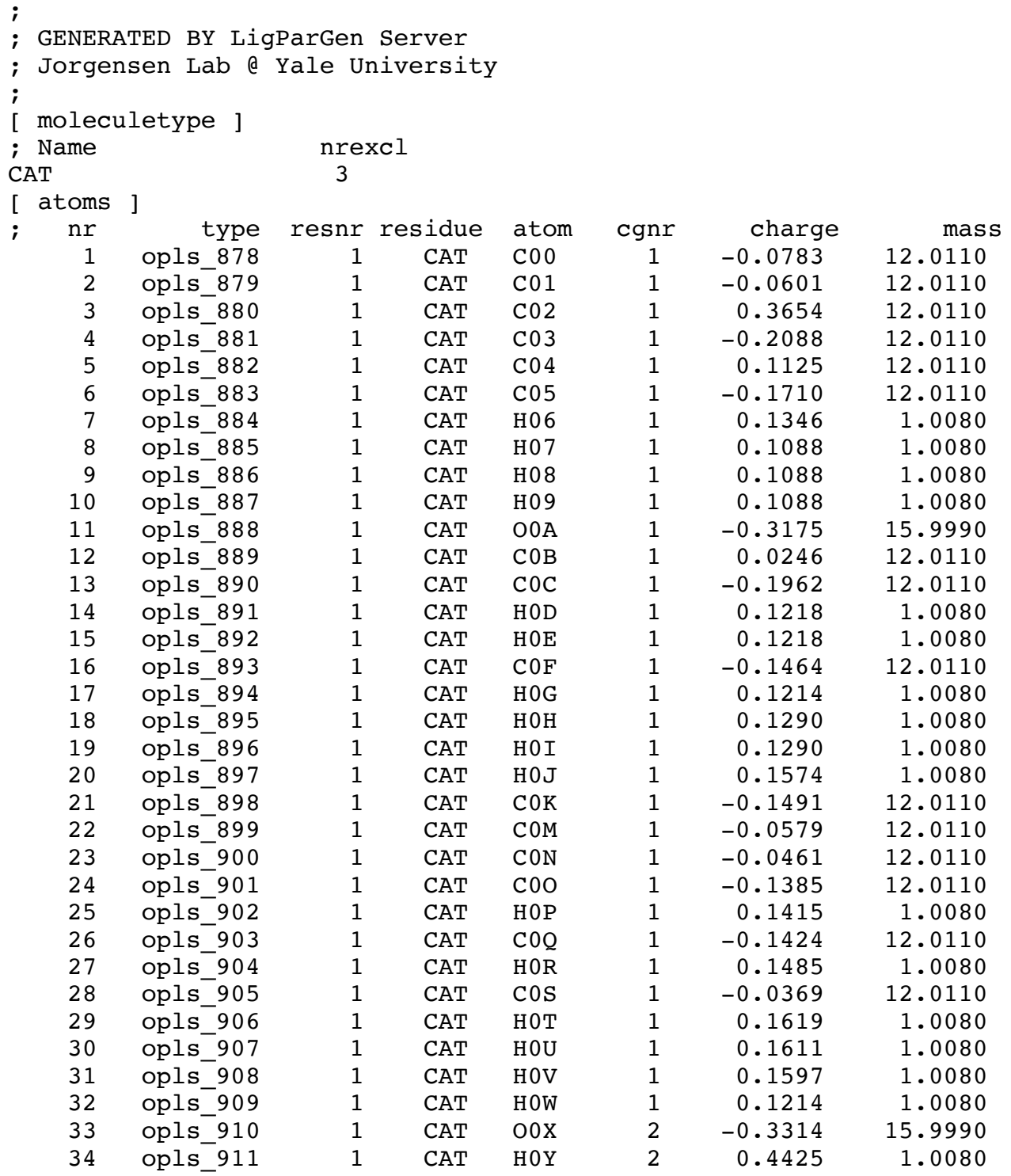

[ bonds ]

$\begin{array}{rrrrr}2 & 1 & 1 & 0.1340 & 459403.200 \\ 3 & 2 & 1 & 0.1460 & 322168.000 \\ 4 & 3 & 1 & 0.1345 & 459403.200 \\ 5 & 4 & 1 & 0.1345 & 459403.200 \\ 6 & 4 & 1 & 0.1510 & 265265.600 \\ 7 & 5 & 1 & 0.1080 & 284512.000 \\ 8 & 6 & 1 & 0.1090 & 284512.000 \\ 9 & 6 & 1 & 0.1090 & 284512.000 \\ 10 & 6 & 1 & 0.1090 & 284512.000 \\ 11 & 2 & 1 & 0.1370 & 376560.000 \\ 12 & 11 & 1 & 0.1410 & 267776.000 \\ 13 & 12 & 1 & 0.1529 & 224262.400 \\ 14 & 12 & 1 & 0.1090 & 284512.000 \\ 15 & 12 & 1 & 0.1090 & 284512.000 \\ 16 & 1 & 1 & 0.1510 & 265265.600 \\ 17 & 13 & 1 & 0.1090 & 284512.000 \\ 18 & 16 & 1 & 0.1090 & 284512.000 \\ 19 & 16 & 1 & 0.1090 & 284512.000\end{array}$




$\begin{array}{rrrrr}20 & 1 & 1 & 0.1080 & 307105.600 \\ 21 & 5 & 1 & 0.1433 & 357313.600 \\ 22 & 21 & 1 & 0.1400 & 392459.200 \\ 23 & 21 & 1 & 0.1400 & 392459.200 \\ 24 & 22 & 1 & 0.1400 & 392459.200 \\ 25 & 22 & 1 & 0.1080 & 307105.600 \\ 26 & 23 & 1 & 0.1400 & 392459.200 \\ 27 & 23 & 1 & 0.1080 & 307105.600 \\ 28 & 24 & 1 & 0.1400 & 392459.200 \\ 29 & 24 & 1 & 0.1080 & 307105.600 \\ 30 & 26 & 1 & 0.1080 & 307105.600 \\ 31 & 28 & 1 & 0.1080 & 307105.600 \\ 32 & 13 & 1 & 0.1090 & 284512.000 \\ 33 & 3 & 1 & 0.1370 & 376560.000 \\ 34 & 33 & 1 & 0.0945 & 462750.400 \\ 16 & 13 & 1 & 0.1529 & 224262.400 \\ 28 & 26 & 1 & 0.1400 & 392459.200\end{array}$

[ angles ]

\begin{tabular}{rrrrrr} 
ai & aj & ak & funct & \multicolumn{3}{c}{ c0 } \\
1 & 2 & 3 & 1 & 124.000 & 585.760 \\
2 & 3 & 4 & 1 & 124.000 & 585.760 \\
3 & 4 & 5 & 1 & 124.000 & 585.760 \\
3 & 4 & 6 & 1 & 124.000 & 585.760 \\
4 & 5 & 7 & 1 & 120.000 & 292.880 \\
4 & 6 & 8 & 1 & 109.500 & 292.880 \\
4 & 6 & 9 & 1 & 109.500 & 292.880 \\
4 & 6 & 10 & 1 & 109.500 & 292.880 \\
1 & 2 & 11 & 1 & 123.000 & 585.760 \\
2 & 11 & 12 & 1 & 111.000 & 627.600 \\
11 & 12 & 13 & 1 & 109.500 & 418.400 \\
11 & 12 & 14 & 1 & 109.500 & 292.880 \\
11 & 12 & 15 & 1 & 109.500 & 292.880 \\
2 & 1 & 16 & 1 & 124.000 & 585.760 \\
12 & 13 & 17 & 1 & 110.700 & 313.800 \\
1 & 16 & 18 & 1 & 109.500 & 292.880 \\
1 & 16 & 19 & 1 & 109.500 & 292.880 \\
2 & 1 & 20 & 1 & 118.760 & 505.678 \\
4 & 5 & 21 & 1 & 117.000 & 711.280 \\
5 & 21 & 22 & 1 & 124.000 & 585.760 \\
5 & 21 & 23 & 1 & 124.000 & 585.760 \\
21 & 22 & 24 & 1 & 120.000 & 527.184 \\
21 & 22 & 25 & 1 & 120.000 & 292.880 \\
21 & 23 & 26 & 1 & 120.000 & 527.184 \\
21 & 23 & 27 & 1 & 120.000 & 292.880 \\
22 & 24 & 28 & 1 & 120.000 & 527.184 \\
22 & 24 & 29 & 1 & 120.000 & 292.880 \\
23 & 26 & 30 & 1 & 120.000 & 292.880 \\
24 & 28 & 31 & 1 & 120.000 & 292.880 \\
12 & 13 & 32 & 1 & 110.700 & 313.800 \\
2 & 3 & 33 & 1 & 123.000 & 585.760 \\
3 & 33 & 34 & 1 & 109.000 & 292.880 \\
5 & 4 & 6 & 1 & 124.000 & 585.760 \\
17 & 13 & 32 & 1 & 107.800 & 276.144 \\
7 & 5 & 21 & 1 & 123.300 & 292.880 \\
12 & 13 & 16 & 1 & 112.700 & 488.273 \\
24 & 22 & 25 & 1 & 120.000 & 292.880 \\
13 & 12 & 14 & 1 & 110.700 & 313.800 \\
22 & 21 & 23 & 1 & 120.000 & 527.184 \\
13 & 16 & 18 & 1 & 110.700 & 313.800 \\
8 & 6 & 10 & 1 & 107.800 & 276.144 \\
18 & 16 & 19 & 1 & 107.800 & 276.144 \\
13 & 12 & 15 & 1 & 110.700 & 313.800 \\
26 & 23 & 27 & 1 & 120.000 & 292.880 \\
28 & 24 & 29 & 1 & 120.000 & 292.880
\end{tabular}




$\begin{array}{rrrrrr}16 & 13 & 32 & 1 & 110.700 & 313.800 \\ 28 & 26 & 30 & 1 & 120.000 & 292.880 \\ 1 & 16 & 13 & 1 & 111.100 & 527.184 \\ 3 & 2 & 11 & 1 & 123.000 & 585.760 \\ 14 & 12 & 15 & 1 & 107.800 & 276.144 \\ 16 & 13 & 17 & 1 & 110.700 & 313.800 \\ 4 & 3 & 33 & 1 & 123.000 & 585.760 \\ 8 & 6 & 9 & 1 & 107.800 & 276.144 \\ 23 & 26 & 28 & 1 & 120.000 & 527.184 \\ 9 & 6 & 10 & 1 & 107.800 & 276.144 \\ 26 & 28 & 31 & 1 & 120.000 & 292.880 \\ 24 & 28 & 26 & 1 & 120.000 & 527.184 \\ 13 & 16 & 19 & 1 & 110.700 & 313.800 \\ 16 & 1 & 20 & 1 & 118.760 & 505.678\end{array}$

[ dihedrals ]

; IMPROPER DIHEDRAL ANGLES

\begin{tabular}{|c|c|c|c|c|}
\hline$i_{c 4} \quad$ ai & $a j$ & $\begin{array}{l}\text { ak } \\
5\end{array}$ & al & Eunct \\
\hline 21 & 5 & 4 & 7 & 4 \\
\hline 23 & 21 & 5 & 22 & 4 \\
\hline 6 & 4 & 3 & 5 & 4 \\
\hline 29 & 24 & 22 & 28 & 4 \\
\hline 27 & 23 & 21 & 26 & 4 \\
\hline 25 & 22 & 21 & 24 & 4 \\
\hline 31 & 28 & 24 & 26 & 4 \\
\hline 30 & 26 & 23 & 28 & 4 \\
\hline 20 & 1 & 2 & 16 & 4 \\
\hline 33 & 3 & 2 & 4 & 4 \\
\hline 11 & 2 & 1 & 3 & 4 \\
\hline
\end{tabular}

$\mathrm{C} 0$

180.000
180.000
180.000
180.000
180.000
180.000
180.000
180.000
180.000
180.000
180.000

C1

$\begin{array}{ll}10.460 & 2 \\ 10.460 & 2 \\ 10.460 & 2 \\ 10.460 & 2 \\ 10.460 & 2 \\ 10.460 & 2 \\ 10.460 & 2 \\ 10.460 & 2 \\ 10.460 & 2 \\ 10.460 & 2 \\ 10.460 & 2\end{array}$

[ dihedrals ]

; PROPER DIHEDRAL ANGLES

\begin{tabular}{|c|c|c|c|c|c|c|c|c|c|c|}
\hline$\underset{c 4}{i} \quad a i$ & $a j$ & $\begin{array}{l}\text { ak } \\
\text { c5 }\end{array}$ & & & & & C1 & & C2 & \\
\hline 5 & 4 & 3 & 2 & 3 & 58.576 & 0.000 & -58.576 & -0.000 & -0.000 & 0.000 \\
\hline 4 & 3 & 2 & 1 & 3 & 21.738 & 2.408 & -16.966 & -7.180 & -0.000 & 0.000 \\
\hline 21 & 5 & 4 & 3 & 3 & 58.576 & 0.000 & -58.576 & -0.000 & -0.000 & 0.000 \\
\hline 21 & 5 & 4 & 6 & 3 & 58.576 & 0.000 & -58.576 & -0.000 & -0.000 & 0.000 \\
\hline 23 & 21 & 5 & 4 & 3 & 16.027 & -4.391 & -14.029 & 2.393 & -0.000 & 0.000 \\
\hline 22 & 21 & 5 & 4 & 3 & 16.027 & -4.391 & -14.029 & 2.393 & -0.000 & 0.000 \\
\hline 22 & 21 & 5 & 7 & 3 & -0.778 & -2.335 & 0.000 & 3.113 & -0.000 & 0.000 \\
\hline 23 & 21 & 5 & 7 & 3 & -0.778 & -2.335 & 0.000 & 3.113 & -0.000 & 0.000 \\
\hline 24 & 22 & 21 & 5 & 3 & 30.334 & 0.000 & -30.334 & -0.000 & -0.000 & 0.000 \\
\hline 26 & 23 & 21 & 5 & 3 & 30.334 & 0.000 & -30.334 & -0.000 & -0.000 & 0.000 \\
\hline 28 & 26 & 23 & 21 & 3 & 30.334 & 0.000 & -30.334 & -0.000 & -0.000 & 0.000 \\
\hline 24 & 28 & 26 & 23 & 3 & 30.334 & 0.000 & -30.334 & -0.000 & -0.000 & 0.000 \\
\hline 24 & 22 & 21 & 23 & 3 & 30.334 & 0.000 & -30.334 & -0.000 & -0.000 & 0.000 \\
\hline 28 & 24 & 22 & 21 & 3 & 30.334 & 0.000 & -30.334 & -0.000 & -0.000 & 0.000 \\
\hline 26 & 28 & 24 & 22 & 3 & 30.334 & 0.000 & -30.334 & -0.000 & -0.000 & 0.000 \\
\hline 26 & 23 & 21 & 22 & 3 & 30.334 & 0.000 & -30.334 & -0.000 & -0.000 & 0.000 \\
\hline 28 & 26 & 23 & 27 & 3 & 30.334 & 0.000 & -30.334 & -0.000 & -0.000 & 0.000 \\
\hline 28 & 24 & 22 & 25 & 3 & 30.334 & 0.000 & -30.334 & -0.000 & -0.000 & 0.000 \\
\hline 6 & 4 & 3 & 2 & 3 & 58.576 & 0.000 & -58.576 & -0.000 & -0.000 & 0.000 \\
\hline 16 & 1 & 2 & 3 & 3 & 58.576 & 0.000 & -58.576 & -0.000 & -0.000 & 0.000 \\
\hline 16 & 1 & 2 & 11 & 3 & 58.576 & 0.000 & -58.576 & -0.000 & -0.000 & 0.000 \\
\hline 13 & 16 & 1 & 2 & 3 & 0.527 & -6.397 & -1.695 & 7.565 & -0.000 & 0.000 \\
\hline 12 & 13 & 16 & 1 & 3 & 2.301 & -1.464 & 0.837 & -1.674 & -0.000 & 0.000 \\
\hline 16 & 13 & 12 & 14 & 3 & 0.628 & 1.883 & 0.000 & -2.510 & -0.000 & 0.000 \\
\hline 16 & 13 & 12 & 15 & 3 & 0.628 & 1.883 & 0.000 & -2.510 & -0.000 & 0.000 \\
\hline 16 & 13 & 12 & 11 & 3 & 2.874 & 0.582 & 2.092 & -5.548 & -0.000 & 0.000 \\
\hline 13 & 12 & 11 & 2 & 3 & 1.715 & 2.845 & 1.046 & -5.607 & -0.000 & 0.000 \\
\hline 12 & 11 & 2 & 3 & 3 & 1.715 & 2.845 & 1.046 & -5.607 & -0.000 & 0.000 \\
\hline 12 & 11 & 2 & 1 & 3 & 5.230 & 7.322 & -12.552 & -0.000 & -0.000 & 0.000 \\
\hline 27 & 23 & 21 & 5 & 3 & 30.334 & 0.000 & -30.334 & -0.000 & -0.000 & 0.000 \\
\hline
\end{tabular}




\begin{tabular}{|c|c|c|c|c|c|c|c|c|c|c|}
\hline 25 & 22 & 21 & 5 & 3 & 30.334 & 0.000 & -30.334 & -0.000 & -0.000 & 0.000 \\
\hline 27 & 23 & 21 & 22 & 3 & 30.334 & 0.000 & -30.334 & -0.000 & -0.000 & 0.000 \\
\hline 30 & 26 & 23 & 21 & 3 & 30.334 & 0.000 & -30.334 & -0.000 & -0.000 & 0.000 \\
\hline 31 & 28 & 26 & 23 & 3 & 30.334 & 0.000 & -30.334 & -0.000 & -0.000 & 0.000 \\
\hline 31 & 28 & 24 & 22 & 3 & 30.334 & 0.000 & -30.334 & -0.000 & -0.000 & 0.000 \\
\hline 30 & 26 & 28 & 24 & 3 & 30.334 & 0.000 & -30.334 & -0.000 & -0.000 & 0.000 \\
\hline 29 & 24 & 22 & 21 & 3 & 30.334 & 0.000 & -30.334 & -0.000 & -0.000 & 0.000 \\
\hline 29 & 24 & 28 & 26 & 3 & 30.334 & 0.000 & -30.334 & -0.000 & -0.000 & 0.000 \\
\hline 25 & 22 & 21 & 23 & 3 & 30.334 & 0.000 & -30.334 & -0.000 & -0.000 & 0.000 \\
\hline 31 & 28 & 26 & 30 & 3 & 30.334 & 0.000 & -30.334 & -0.000 & -0.000 & 0.000 \\
\hline 31 & 28 & 24 & 29 & 3 & 30.334 & 0.000 & -30.334 & -0.000 & -0.000 & 0.000 \\
\hline 30 & 26 & 23 & 27 & 3 & 30.334 & 0.000 & -30.334 & -0.000 & -0.000 & 0.000 \\
\hline 29 & 24 & 22 & 25 & 3 & 30.334 & 0.000 & -30.334 & -0.000 & -0.000 & 0.000 \\
\hline 20 & 1 & 2 & 3 & 3 & 58.576 & 0.000 & -58.576 & -0.000 & -0.000 & 0.000 \\
\hline 20 & 1 & 2 & 11 & 3 & 58.576 & 0.000 & -58.576 & -0.000 & -0.000 & 0.000 \\
\hline 20 & 1 & 16 & 13 & 3 & -33.472 & 0.000 & 33.472 & -0.000 & -0.000 & 0.000 \\
\hline 20 & 1 & 16 & 19 & 3 & 0.665 & 1.996 & 0.000 & -2.661 & -0.000 & 0.000 \\
\hline 20 & 1 & 16 & 18 & 3 & 0.665 & 1.996 & 0.000 & -2.661 & -0.000 & 0.000 \\
\hline 7 & 5 & 4 & 3 & 3 & 58.576 & 0.000 & -58.576 & -0.000 & -0.000 & 0.000 \\
\hline 7 & 5 & 4 & 6 & 3 & 58.576 & 0.000 & -58.576 & -0.000 & -0.000 & 0.000 \\
\hline 9 & 6 & 4 & 5 & 3 & -0.778 & -2.335 & 0.000 & 3.113 & -0.000 & 0.000 \\
\hline 9 & 6 & 4 & 3 & 3 & -0.778 & -2.335 & 0.000 & 3.113 & -0.000 & 0.000 \\
\hline 10 & 6 & 4 & 3 & 3 & -0.778 & -2.335 & 0.000 & 3.113 & -0.000 & 0.000 \\
\hline 8 & 6 & 4 & 5 & 3 & -0.778 & -2.335 & 0.000 & 3.113 & -0.000 & 0.000 \\
\hline 8 & 6 & 4 & 3 & 3 & -0.778 & -2.335 & 0.000 & 3.113 & -0.000 & 0.000 \\
\hline 10 & 6 & 4 & 5 & 3 & -0.778 & -2.335 & 0.000 & 3.113 & -0.000 & 0.000 \\
\hline 18 & 16 & 1 & 2 & 3 & -0.778 & -2.335 & 0.000 & 3.113 & -0.000 & 0.000 \\
\hline 19 & 16 & 1 & 2 & 3 & -0.778 & -2.335 & 0.000 & 3.113 & -0.000 & 0.000 \\
\hline 32 & 13 & 16 & 1 & 3 & 0.766 & 2.297 & 0.000 & -3.063 & -0.000 & 0.000 \\
\hline 17 & 13 & 16 & 1 & 3 & 0.766 & 2.297 & 0.000 & -3.063 & -0.000 & 0.000 \\
\hline 19 & 16 & 13 & 12 & 3 & 0.628 & 1.883 & 0.000 & -2.510 & -0.000 & 0.000 \\
\hline 18 & 16 & 13 & 12 & 3 & 0.628 & 1.883 & 0.000 & -2.510 & -0.000 & 0.000 \\
\hline 17 & 13 & 12 & 14 & 3 & 0.628 & 1.883 & 0.000 & -2.510 & -0.000 & 0.000 \\
\hline 18 & 16 & 13 & 17 & 3 & 0.628 & 1.883 & 0.000 & -2.510 & -0.000 & 0.000 \\
\hline 32 & 13 & 16 & 18 & 3 & 0.628 & 1.883 & 0.000 & -2.510 & -0.000 & 0.000 \\
\hline 19 & 16 & 13 & 17 & 3 & 0.628 & 1.883 & 0.000 & -2.510 & -0.000 & 0.000 \\
\hline 32 & 13 & 12 & 14 & 3 & 0.628 & 1.883 & 0.000 & -2.510 & -0.000 & 0.000 \\
\hline 17 & 13 & 12 & 15 & 3 & 0.628 & 1.883 & 0.000 & -2.510 & -0.000 & 0.000 \\
\hline 32 & 13 & 16 & 19 & 3 & 0.628 & 1.883 & 0.000 & -2.510 & -0.000 & 0.000 \\
\hline 32 & 13 & 12 & 15 & 3 & 0.628 & 1.883 & 0.000 & -2.510 & -0.000 & 0.000 \\
\hline 32 & 13 & 12 & 11 & 3 & 0.979 & 2.937 & 0.000 & -3.916 & -0.000 & 0.000 \\
\hline 17 & 13 & 12 & 11 & 3 & 0.979 & 2.937 & 0.000 & -3.916 & -0.000 & 0.000 \\
\hline 15 & 12 & 11 & 2 & 3 & 1.590 & 4.770 & 0.000 & -6.360 & -0.000 & 0.000 \\
\hline 14 & 12 & 11 & 2 & 3 & 1.590 & 4.770 & 0.000 & -6.360 & -0.000 & 0.000 \\
\hline 34 & 33 & 3 & 4 & 3 & -0.444 & 3.833 & 0.728 & -4.117 & -0.000 & 0.000 \\
\hline 34 & 33 & 3 & 2 & 3 & -1.883 & 1.883 & 0.000 & -0.000 & -0.000 & 0.000 \\
\hline 33 & 3 & 4 & 5 & 3 & -5.858 & -6.694 & 12.552 & -0.000 & -0.000 & 0.000 \\
\hline 33 & 3 & 4 & 6 & 3 & -5.858 & -6.694 & 12.552 & -0.000 & -0.000 & 0.000 \\
\hline 33 & 3 & 2 & 1 & 3 & -5.858 & -6.694 & 12.552 & -0.000 & -0.000 & 0.000 \\
\hline 33 & 3 & 2 & 11 & 3 & 9.035 & -9.035 & 0.000 & -0.000 & -0.000 & 0.000 \\
\hline 11 & 2 & 3 & 4 & 3 & -0.778 & -2.335 & 0.000 & 3.113 & -0.000 & 0.000 \\
\hline
\end{tabular}

$\begin{array}{crl}\text { pairs ] } & & \\ 1 & 4 & 1 \\ 2 & 5 & 1 \\ 2 & 6 & 1 \\ 3 & 7 & 1 \\ 3 & 8 & 1 \\ 3 & 9 & 1 \\ 6 & 7 & 1 \\ 5 & 8 & 1 \\ 3 & 10 & 1 \\ 1 & 12 & 1 \\ 5 & 9 & 1 \\ 5 & 10 & 1\end{array}$




$\begin{array}{rll}4 & 11 & 1 \\ 3 & 12 & 1 \\ 2 & 13 & 1 \\ 2 & 14 & 1 \\ 2 & 15 & 1 \\ 1 & 17 & 1 \\ 3 & 16 & 1 \\ 2 & 18 & 1 \\ 2 & 19 & 1 \\ 3 & 20 & 1 \\ 3 & 21 & 1 \\ 4 & 22 & 1 \\ 11 & 16 & 1 \\ 6 & 21 & 1 \\ 4 & 23 & 1 \\ 11 & 17 & 1 \\ 7 & 22 & 1 \\ 5 & 24 & 1 \\ 14 & 16 & 1 \\ 12 & 18 & 1 \\ 7 & 23 & 1 \\ 5 & 25 & 1 \\ 15 & 16 & 1 \\ 14 & 17 & 1 \\ 12 & 19 & 1 \\ 11 & 20 & 1 \\ 5 & 26 & 1 \\ 15 & 17 & 1 \\ 5 & 27 & 1 \\ 13 & 20 & 1 \\ 1 & 32 & 1 \\ 1 & 33 & 1 \\ 17 & 18 & 1 \\ 17 & 19 & 1 \\ 2 & 34 & 1 \\ 18 & 20 & 1 \\ 5 & 33 & 1 \\ 4 & 34 & 1 \\ 19 & 20 & 1 \\ 6 & 33 & 1 \\ 11 & 32 & 1 \\ 11 & 33 & 1 \\ 14 & 32 & 1 \\ 23 & 24 & 1 \\ 15 & 32 & 1 \\ 23 & 25 & 1 \\ 22 & 26 & 1 \\ 22 & 27 & 1 \\ 21 & 28 & 1 \\ 21 & 29 & 1 \\ 18 & 32 & 1 \\ 21 & 30 & 1 \\ 19 & 32 & 1 \\ 25 & 28 & 1 \\ 22 & 31 & 1 \\ 25 & 29 & 1 \\ 24 & 30 & 1 \\ 27 & 31 & 1 \\ 26 & 29 & 1 \\ 27 & 30 & 1 \\ 29 & 31 & 1 \\ 30 & 31 & 1\end{array}$




\section{Plots of P-O Distance vs Time for MD Simulations of Ion Pair 7}
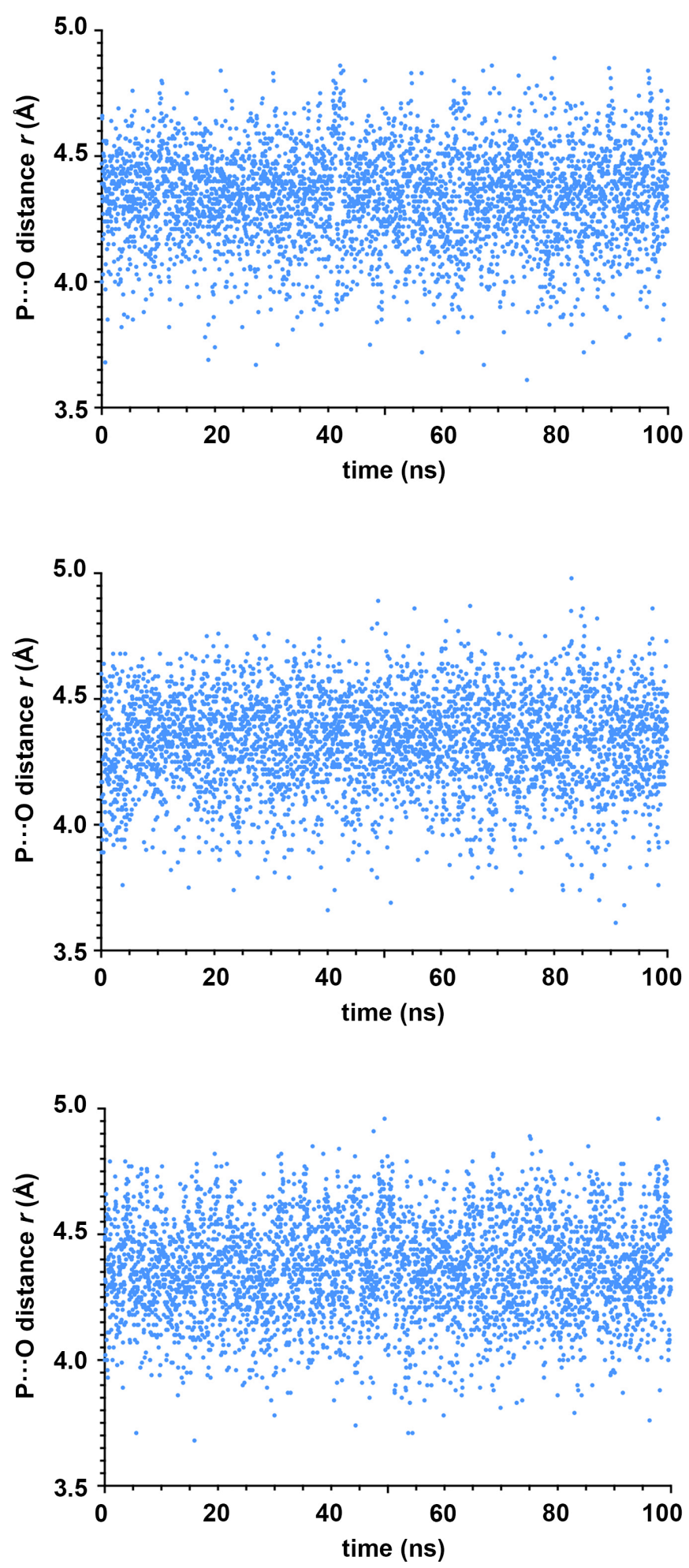

Figure S4. Variation in inter-ion distance over time during three $100 \mathrm{~ns}$ molecular dynamics simulations of the ion pair 7 in chloroform. These plots are analogous to the one shown in Figure 6 of the paper, but they show the other three of the four 100 ns simulations that were performed. 


\section{Computations of the Reactions Shown in Figure 8 of the Paper}

Table S7. Computed Energies of the Transition States for the Reactions Shown in Figure 8 of the Paper

\begin{tabular}{|lccc|}
\hline Single point calculation method & $\begin{array}{c}\boldsymbol{E} \text { of lower- } \\
\text { energy TS } \\
\text { (Hartree) }\end{array}$ & $\begin{array}{c}\boldsymbol{E} \text { of higher- } \\
\text { energy TS } \\
\text { (Hartree) }\end{array}$ & $\begin{array}{c}\Delta \boldsymbol{\Delta} \boldsymbol{E}^{\ddagger} \\
(\mathrm{kcal} / \mathrm{mol})\end{array}$ \\
\hline Reaction 1 & & & \\
M06-2X/6-311+G(d,p) & -4602.205989 & -4602.201605 & 2.8 \\
M06-2X/6-311+G(d,p)-PCM & -4602.165132 & -4602.160114 & 3.1 \\
M06-2X/def2-TZVPP & -4602.730723 & -4602.726614 & 2.6 \\
M06-2X-D3/6-311+G(d,p) & -4602.228173 & -4602.223023 & 3.2 \\
B3LYP-D3(BJ)/6-311+G(d,p) & -4604.139384 & -4604.135250 & 2.6 \\
B97-D/6-311+G(d,p) & -4601.061732 & -4601.057330 & 2.8 \\
Reaction 24 & & & \\
M06-2X/6-311+G(d,p) & -6471.128195 & -6471.123320 & 3.1 \\
M06-2X/6-311+G(d,p)-PCM & -6471.072519 & -6471.066503 & 3.8 \\
M06-2X/def2-TZVPP & -6471.837946 & -6471.833545 & 2.8 \\
M06-2X-D3/6-311+G(d,p) & -6471.165256 & -6471.159875 & 3.4 \\
B3LYP-D3(BJ)/6-311+G(d,p) & -6473.528760 & -6473.524534 & 2.7 \\
B97-D/6-311+G(d,p) & -6469.880299 & -6469.878701 & 1.0 \\
\hline
\end{tabular}

${ }^{a}$ Unless otherwise stated, the single-point calculations used the SMD model of chloroform. The TS geometries were obtained from the references cited. 


\section{References}

1. Rueping, M.; Ieawsuwan, W.; Antonchick, A. P.; Nachtsheim, B. J. Chiral Brønsted Acids in the Catalytic Asymmetric Nazarov Cyclization-The First Enantioselective Organocatalytic Electrocyclic Reaction. Angew. Chem. Int. Ed. 2007, 46, 2097-2100.

2. Stephens, P. J.; Devlin, F. J.; Cheeseman, J. R.; Frisch, M. J. Calculation of Optical Rotation Using Density Functional Theory. J. Phys. Chem. A 2001, 105, 5356-5371.

3. Simón, L.; Goodman, J. M. Theoretical Study of the Mechanism of Hantzsch Ester Hydrogenation of Imines Catalyzed by Chiral BINOL-Phosphoric Acids. J. Am. Chem. Soc. 2008, 130, 8741-8747.

4. Seguin, T. J.; Wheeler, S. E. Stacking and Electrostatic Interactions Drive the Stereoselectivity of Silylium-Ion Asymmetric Counteranion-Directed Catalysis. Angew. Chem. Int. Ed. 2016, 55, 15889-15893. 